\title{
Durchführung der Konzeptstudie
}

\subsection{Anforderungsanalyse}

Die Klärung und Präzisierung der Aufgabenstellung erfolgte in Kapitel 1, in welchem die Motivation für die Erarbeitung eines Konzepts für variable PitotEinlässe für zukünftige Flugtriebwerke dargelegt wurde. Folgende Teilaufgaben waren während der Erarbeitung des Konzepts zu erfüllen:

- Ermitteln des geeigneten Anwendungs- und Geschwindigkeitsbereichs innerhalb der Luftfahrt,

- Erarbeiten eines Konzepts, das perspektivisch industrielle Standards für Sicherheit und Zuverlässigkeit erfüllen kann, sowie

- Aufzeigen der prinzipiellen Umsetzbarkeit des Konzepts und seines Potenzials für die Luftfahrt, wodurch Reifegrad TRL 3 erreicht wird.

\subsubsection{Erarbeitung der Anforderungsliste}

Im direkten Anschluss an die Klärung der Aufgabenstellung wurde eine erste Anforderungsliste erarbeitet. Diese Anforderungsliste war mit der ersten Erstellung allerdings nicht vollständig abgeschlossen. Aufgrund von Ergänzungen durch Zwischenergebnisse im Verlauf der Konzeptstudie erfolgten mehrere Iterationen. Einige dieser Iterationen können Veröffentlichungen [310], [311] und studentischen Abschlussarbeiten [312], [313], [314], [315] entnommen werden. Die abschließende Version der Anforderungsliste ist in Anhang A.2 zu finden. Nachfolgend wird deren Herleitung vorgestellt. 


\section{Ermitteln der Anforderungsquellen}

Für die Entwicklung variabler Triebwerkseinlässe sind zahlreiche primäre Anforderungsquellen in Betracht zu ziehen. $\mathrm{Zu}$ diesen Quellen, die direkt durch den Einlass beeinflusst werden können, zählen:

- Piloten,

- Passagiere und Kabinenbesatzung,

- Triebwerks-, Flugzeughersteller und Zulieferer,

- Wartungsunternehmen,

- Flugzeugbetreiber oder Fluggesellschaften,

- Flughafenbetreiber,

- Anwohner im Bereich des Flughafens sowie

- Luftfahrtbehörden und Gesetzgeber.

\section{Ermittlung der Anforderungen}

Mit Hilfe von Brainstorming-Methoden wurden die Anforderungen aus der Perspektive der jeweiligen Anforderungsquelle identifiziert. Anschließend erfolgte eine Ergänzung der ermittelten Anforderungen durch Recherche der Fachliteratur, vgl. Rolls-Royce [66, S. 30], Farokhi [47, S. 327-328], Sadraey [284, S. 38]. Um weitere relevante Anforderungen zu bestimmen, wurden Checklisten eingesetzt, vgl. Abschnitt 3.2.1. Die gesammelten Anforderungen wurden in einer strukturierten Anforderungsliste zusammengetragen, vgl. Anhang A.2.

Falls möglich, sind den Anforderungen Zielwerte zugeordnet worden. Ebenso erfolgte eine Unterteilung in Festforderungen und Wünsche. Diese Unterteilung erleichtert die Festlegung von Ausschlusskriterien. Weiterhin wurde die Anforderungsliste um Anforderungen bereinigt, welche für die Erarbeitung eines Konzepts bis Reifegrad TRL 3 vernachlässigbar sind, beispielsweise optische Eigenschaften des Einlasses oder Lärmemissionen eines möglichen Stellsystems.

Die Erstellung einer strukturierten Anforderungsliste unterstützt die spätere Identifikation der Bewertungskriterien. Darauf aufbauend wurden die identifizierten Anforderungen für variable Pitot-Einlässe den folgenden konstruktionstechnischen Kategorien zugeordnet:

- aerodynamische Funktionalität,

- Integrierbarkeit,

- Masse und Widerstand gegen Beanspruchungen,

- Sicherheit,

- Zuverlässigkeit und Lebenswegkosten sowie 
- Entwicklungs- und Produktionskosten [47, S. 327-328], [66, S. 30], [284, S. 33-38].

\section{Aerodynamische Funktionalität}

Die Kategorie der aerodynamischen Funktionalität des variablen Einlasssystems beinhaltet den gewünschten Betriebsbereich, die Geometrieanforderungen sowie die erforderlichen Werte für die aerodynamischen Kennzahlen.

Zum Betriebsbereich zählen die bei verschiedenen Fluggeschwindigkeiten bis Mach 1,6 vom Triebwerk geforderten Luftmassenströme $\dot{m}_{0}$. Die Luftmassenströme können unter anderem näherungsweise mit der Kontinuitätsgleichung aus dem Produkt der Dichte der anströmenden Luft $\rho_{2}$, der vom Fan geforderten Anströmgeschwindigkeit $c_{2}$ und des Strömungsquerschnitts in der Fanebene $A_{2}$ bestimmt werden, vgl. Wöllner [315, S. 47-49].

Die aerodynamische Funktionalität eines variablen Einlasskonzepts wird vorrangig durch seine einstellbaren Geometrien bestimmt. Als Ausgangspunkt für die Größenordnung des zu entwickelnden Einlasskonzeptes dienten die geometrischen Abmessungen von Einlass und Fan der BR-700-Triebwerksreihe von Rolls-Royce, die beispielsweise in den Geschäftsreiseflugzeugen Gulfstream G650 eingesetzt wird [316]. Basierend auf dem Fandurchmesser dieser Triebwerksreihe wurden nach Bräunling [10, S. 967-980] zunächst auf analytischem Weg überschlägige ideale Geometrien ermittelt. Die Berechnung dieser Geometrien erfolgte ausschließlich für den Unterschallbetrieb bis Mach 0,95, vgl. Abbildung 4.1.

Wenngleich diese rudimentär identifizierten Geometrien noch keinen Einsatz in einem realen Triebwerk zulassen würden, geben sie einen grundlegenden Eindruck über die vom variablen Einlass umzusetzende Geometrievariation. Zudem fanden die Geometrien bei der ersten Erstellung von Konzepten und im ersten von zwei Funktionsdemonstratoren Verwendung, vgl. Abschnitt 4.3.1 und 4.5.4.

Im weiteren Verlauf offenbarten erste numerische Strömungsuntersuchungen dieser Geometrien deren geringes aerodynamisches Einsparpotenzial. Dies ist sowohl mit dem rudimentären Charakter der Geometrien als auch mit dem allgemeinhin geringen Einsparpotenzial im reinen Unterschallbetrieb begründbar. Das geringe Potenzial wird von Baier [21, S. 2] mit einer um $5 \%$ reduzierbaren mitzuführenden Kraftstoffmenge quantifiziert. Dieser Ersparnis stehen die genannten Herausforderungen bezüglich Zuverlässigkeit, Sicherheit sowie Entwicklungs-, Herstellungs- und Wartungskosten gegenüber. Deshalb wurden im weiteren Verlauf der Konzeptstudie variable Pitot-Einlässe für den Überschallbetrieb bis Mach 1,6 untersucht und im späteren Verlauf deren ideale Geometrien und das zugehörige Einsparpotenzial detailliert ermittelt, vgl. Abschnitt 4.4.3. 


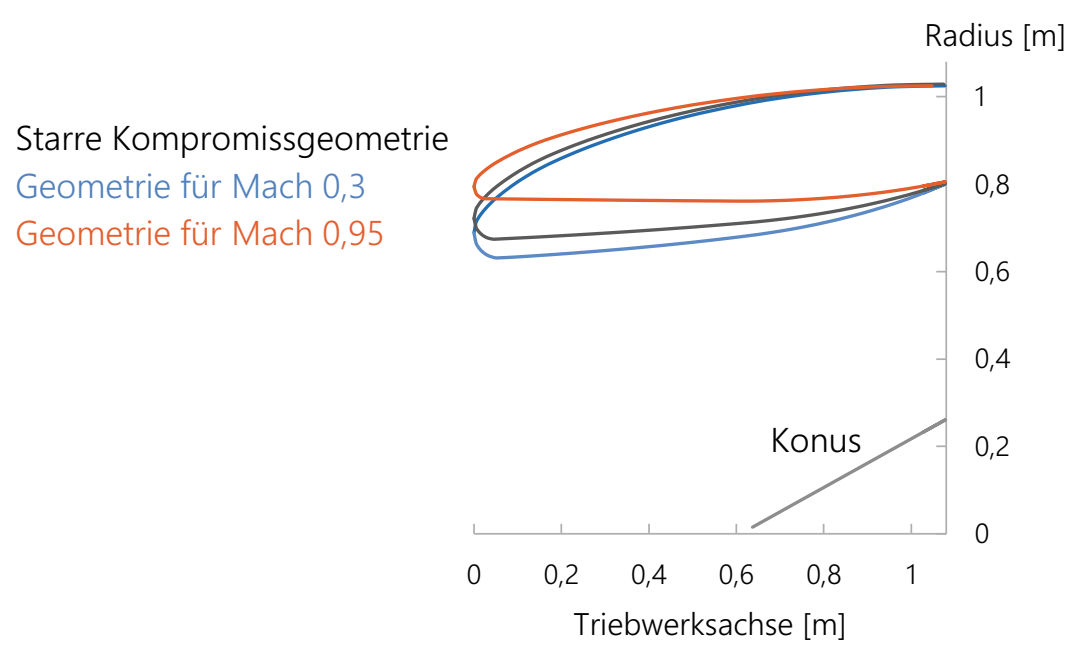

Abbildung 4.1 Analytisch ermittelte Einlassgeometrien

$\mathrm{Zu}$ den weiteren aerodynamischen Anforderungen zählen Beschränkungen der relativen Abweichungen der vom variablen Einlasskonzept umgesetzten Geometrie im Vergleich zur idealen Geometrie. Diese Abweichungen können im Bereich der Konturglätte, der Rundheit, der Durchmessereinstellbarkeit oder der Lippendicke auftreten. Auch Stufen, Spalte und die Oberflächenqualität beeinflussen die aerodynamische Funktionalität des Einlasses.

Weiterhin sind vom variablen System akzeptable Wertebereiche für die aerodynamischen Kennzahlen des Einlasses zu gewährleisten. Mit dem Einlassdruckverhältnis $\pi_{i n l}$, dem $D C(60)$-Koeffizienten als Maß der Gleichförmigkeit der Fananströmung und dem externen Gondelvorkörperwiderstand (Überlaufwiderstand) $D_{\text {nacf,ext }}$ wurden die wichtigsten dieser Kennzahlen in Abschnitt 2.3.1 vorgestellt.

\section{Integrierbarkeit}

Die Integrierbarkeit eines Systems hängt vorrangig vom benötigten Bauraum der Systemkomponenten und ihren Anforderungen ab. Auch mögliche Schnittstellen, Synergien und Interaktionen zwischen Komponenten innerhalb der Einlasszone sowie in der direkten Umgebung des Einlasses sind für die Integrierbarkeit von großer Bedeutung. Dabei sollte die Nutzung möglicher Synergien zwischen den 
verschiedenen potenziellen Teilsystemen maximiert werden, z. B. durch eine gemeinsame Verwendung von Komponenten.

Der vorhandene Bauraum innerhalb des variablen Einlasses wird durch seine minimalen Konturabmessungen bestimmt. Die minimale Länge des Einlasses wird dabei durch die Unterschall-Geometrie festgelegt, während die minimale Dicke bei der Überschall-Kontur erforderlich ist. Zu beachten ist, dass Teilsysteme innerhalb des Einlasses zu keinem Betriebszeitpunkt die Strömung negativ beeinflussen dürfen und somit vollständig innerhalb der Einlaufstruktur befindlich verbleiben müssen.

$\mathrm{Zu}$ den Komponenten, die innerhalb der Einlasszone zu integrieren sind, zählen beispielsweise Eisschutzsysteme, akustische Auskleidungen, Messsonden oder ein mögliches Stellsystem, vgl. Abschnitt 2.3. Anforderungen an ein Stellsystem können beispielsweise hinsichtlich Bewegungsart, -richtung, Stellzeit, -weg, -toleranzen, -kräften oder Energiebedarf bestehen. In der direkten Umgebung des Einlasses ist insbesondere der Fan von Bedeutung, wobei sicherheitskritische Interaktionen mit diesem zu vermeiden sind.

Wechselwirkungen zwischen den Komponenten innerhalb der Einlasszone sowie in der Umgebung des Einlasses werden im Rahmen der Zonensicherheitsanalyse (ZSA) untersucht, vgl. Abschnitt 4.4.1. So können beispielsweise heiße Oberflächen von Eisschutzsystemen in Verbindung mit brennbaren Flüssigkeiten des Stellsystems im Falle einer Leckage zu einem Feuer führen. Die Minderung dieses Risikos kann konstruktive Maßnahmen erfordern. Zu möglichen Maßnahmen zählen die Ventilation der Luft, die Ableitung von Flüssigkeiten, die Integration von Schottwänden, der Einbau von Feuerdetektionsmechanismen sowie ein Feuerlöschsystem innerhalb der Einlassstruktur. Darüber hinaus sind Schäden an elektrischen Komponenten, beispielsweise dem Stellsystem oder Sensoren, durch das Austreten heißer Luft oder anderer Flüssigkeiten infolge einer Leckage in der Nähe zu vermeiden. Folglich könnten Überdruckausgleichklappen, Ventilation, Drainage und Hitzeschutz für das Stellsystem erforderlich werden. Mögliche elektrische Komponenten müssen durch Erdung, Integration von Drainage und wasserdichte Bauteilverkleidungen vor Beschädigungen geschützt werden. Durch eine mechanische Anpassung der Einlassgeometrie können zusätzliche Reibung zwischen Komponenten, erhöhte Spannungen und dadurch Schäden an starren Kabeln und Leitungen auftreten. Diese lassen sich durch eine flexible Gestaltung der Verkabelung und der Leitungsinstallation sowie einen Mindestabstand zwischen den Komponenten vermeiden. 


\section{Masse und Widerstand gegen Beanspruchungen}

Die Masse des variablen Einlasses ist zu minimieren. Da die Geometrievariation einen Stellmechanismus oder eine andere zusätzliche Technologie erfordert, ist im Vergleich zu starren Einlässen eine größere Gesamtmasse des variablen Systems zu erwarten. Vorrangig wird die Masse des variablen Einlasssystems bestimmt durch

- seine räumlichen Dimensionen,

- die Eigenschaften der verwendeten Materialien,

- zusätzliche Teilsysteme und deren Komponenten, z. B. Aktoren, Leitungen und Verkabelung,

- die erforderlichen Betriebsmittel sowie

- eventuell notwendige Sicherheitsanpassungen, z. B. Schottwände oder Überdruckausgleichklappen.

Die räumlichen Dimensionen des Einlasses hängen einerseits von der erforderlichen aerodynamischen Form und andererseits von den wirkenden Beanspruchungen ab. Die wirkenden Beanspruchungen gehen aus der Umströmung und den vorhandenen Umgebungsbedingungen an den unterschiedlichen Einsatzorten hervor. Dies beinhaltet

- die auftretenden Strömungs- und Windbeanspruchungen,

- den Einfluss von Temperatur und Druck in der Umgebung,

- Niederschläge und hohe Luftfeuchtigkeit,

- Kontakt mit Chemikalien, z. B. Enteisungsfluiden,

- Blitzschläge,

- Sand, Staub- und Schmutzpartikel in der Luft,

- angesaugte Fremdkörper und Vögel sowie

- Reibungswärme im Überschallbetrieb.

In der Folge treten zahlreiche Arten der mechanischen und chemischen Belastung des Einlasses auf. Diese Belastungen werden in Abhängigkeit der Häufigkeit ihres Auftretens und der erlaubten Konsequenzen unterteilt in:

- Ermüdungslasten (Fatigue Loads),

- Grenzlasten (Limit Loads) und

- Extremlasten (Ultimate Loads) [34, 25.301]. 
Ermüdungslasten treten mit hoher Wahrscheinlichkeit bei jedem Flug auf. Diesen Lasten müssen die Komponenten über ihre gesamte Lebensdauer standhalten. Hierzu zählen insbesondere die aerodynamischen Lasten von bis $\mathrm{zu} 2,6 \mathrm{kN}$, die aus der Umströmung des Einlasses resultieren. Die Umströmung des Einlasses wird in Abschnitt 4.4.3 analysiert und die Berechnung der daraus resultierenden Lasten ist Anhang A.1.2 zu entnehmen. Zu den Ermüdungslasten zählen weiterhin thermomechanische Ermüdungslasten, die aus dem unterschiedlichen Wärmeausdehnungsverhalten ungeeigneter Werkstoffpaarungen resultieren können. Darüber hinaus ist der Einlass bei jedem Flug Erosion aufgrund von Sand und Staub in der Luft ausgesetzt.

Grenzlasten können theoretisch bei jedem Flug auf den Einlass wirken, treten in der Praxis jedoch deutlich seltener auf als Ermüdungslasten [34, 25.305]. Wirkenden Grenzlasten muss ohne plastische Verformung oder Beeinträchtigung des sicheren Folgebetriebs standgehalten werden. Hierzu zählen beispielsweise starke Böen, kleinere Fremdkörpereinschläge, harte Landungen und Manöverlasten mit großen Beschleunigungen.

Extremlasten müssen mindestens einmal ertragen werden können, ohne dass ein sicherheitskritisches strukturelles Versagen des variablen Einlasses eintritt [34, 25.305]. Hierzu zählen

- die Belastungen beim Windmilling, sowohl auf den Einlass des betroffenen Triebwerks als auch auf die Einlässe der verbleibenden,

- die Vibrationslasten nach einem Fanschaufelverlust,

- Stoßwellen beim Verdichterpumpen (Hammershocks) [9], [108, S. 289-290] sowie

- Einschläge von Vögeln und kleineren Drohnen.

Variable Einlässe weisen im Vergleich zu starren Einlässen eine veränderte Struktur auf. Diese Struktur muss den auftretenden Lasten dennoch derart standhalten, dass die Sicherheit im Flugbetrieb gewährleistet bleibt. Die größtmöglichen Lasten treten beim Vogelschlag auf und betragen in $95 \%$ aller Fälle weniger als $144 \mathrm{kN}$, vgl. Anhang A.1.1. Dennoch dürfen aus einem einzigen Vogelschlag keine gefährlichen Folgen entstehen. Beispielsweise sollten aufgrund des Vogelschlags keine größeren Bauteile verloren gehen. Zum einen würde die Umströmung des Einlasses und das Betriebsverhalten des Flugzeugs erheblich gestört werden. Zum anderen könnten die besagten Bauteile sicherheitskritische Komponenten, wie zum Beispiel das Leitwerk, beschädigen. Dies ist insbesondere bei Geschäftsreiseflugzeugen, deren Triebwerke vorrangig heckmontiert sind, ein nicht $\mathrm{zu}$ vernachlässigendes Risiko. Dieses Risiko muss klassifiziert und 
durch entsprechende Maßnahmen behandelt werden [34, AMC 25.1309], [282, S. 68-71], [317]. Die von Rochard [318] durchgeführte Risikoanalyse bezüglich Vogelschlag führt beispielsweise die Bedeutung von Vogelvergrämungsmaßnahmen in der Nähe von Flughäfen an. Jedoch kann durch diese Maßnahmen das Risiko des Vogelschlags auf den Einlass nicht vollständig eliminiert werden. Im Rahmen der Auslegung ist somit zu zeigen, dass das Vogelschlagrisiko aufgrund seiner Eintrittswahrscheinlichkeit und der getroffenen Gegenmaßnahmen tolerierbar ist [279, S. 45-47], vgl. Abschnitt 4.5.2.

Darüber hinaus können verschiedene Sonderlasten bei der Montage und dem Transport sowie in Form von Trittlasten während der Wartung auftreten.

\section{Sicherheit}

Entsprechend der potenziellen Auswirkungen möglicher Fehler sind vom Konzept zugehörige Eintrittswahrscheinlichkeiten zu gewährleisten. Grundsätzlich darf ein einzelner Fehler keine gefährlichen Ereignisse nach sich ziehen. Mögliche Ursachen solcher Ereignisse wären Strömungsablösungen, Beschädigungen benachbarter Komponenten und unkontrollierte Feuer. Strömungsablösungen können beispielsweise aufgrund einer ungeeigneten Geometrie bei instationären Flugbedingungen auftreten. Schäden an benachbarten Komponenten können durch abplatzende Eisablagerungen unter Vereisungsbedingungen oder durch verlorene Einlasskomponenten nach einem Vogelschlag verursacht werden. Feuer können aus der Kombination von Zündquellen, brennbaren Fluiden und vorhandenem Sauerstoff resultieren. Weiterhin sind sicherheitskritische Interaktionen zwischen dem Einlass und dem Fan, den Tragflächen, dem Leitwerk sowie dem Flugzeugrumpf zu vermeiden.

Es ist sicherzustellen, dass durch die Variabilität des Einlasses keine gefährlichen Ereignisse verursacht werden. Folglich muss die primäre Funktion des Einlasses, die Luftzufuhr zum Triebwerk, im regulären Betrieb und im Fehlerfall gewährleistet werden. Hierfür muss ein Kontrollsystem für jeden Flugzustand eine geeignete Einlassgeometrie einstellen. Zudem ist der versagenssichere Ausfall des Variationsmechanismus sicherzustellen. Dies bedeutet, dass das variable Einlasssystem bei einem auftretenden Fehler, beispielsweise einem Aktorausfall, in eine Geometrie zurückgeführt werden muss, durch welche Strömungsablösungen vermieden werden.

Darüber hinaus ist die von möglichen Feuern ausgehende Gefahr durch verschiedene konstruktive Vorkehrmaßnahmen zu minimieren. Folglich sind Kombinationen von Zündquellen, brennbaren Fluiden und hohen Sauerstoffkonzentrationen zu vermeiden. Mögliche Zündquellen stellen beispielsweise die heißen Oberflächen eines Enteisungssystems oder Funkenbildung elektrischer 
Komponenten dar. Brennbare Fluide können beispielsweise Betriebsmittel eines möglichen Variationsmechanismus oder Eisschutzsystems sein.

\section{Zuverlässigkeit und Lebenswegkosten}

Die Lebensdauer, die Zuverlässigkeit und die Lebenswegkosten von variablen Einlasskonzepten sind eng miteinander verbunden. Die Lebensdauer der Einlasskomponenten sollte mit der des Triebwerks übereinstimmen. Die Zuverlässigkeit basiert vorrangig auf der Anzahl der Teile und der Widerstandsfähigkeit gegen Erosion, Ermüdung und Grenzbelastungen. Die Bauteilanzahl und die Komplexität der Konstruktion sind folglich $\mathrm{zu}$ minimieren. Darüber hinaus darf auftretende Feuchtigkeit nicht zu Korrosion führen. Mögliche Spalte, Öffnungen und Ventile sind vor Verstopfen durch Sand und Schmutz zu schützen. Reibung zwischen beweglichen Teilen kann die Konturvariation behindern oder Komponenten beschädigen und ist $\mathrm{zu}$ minimieren. Außerdem sollten Chemikalien, z. B. Enteisungsfluide, die Lebensdauer der verwendeten Materialien nicht negativ beeinflussen.

Die Lebenswegkosten variabler Einlässe werden zusätzlich durch deren Leistungsbedarf, notwendige Verbrauchsmaterialien, z. B. Schmierstoffe, sowie Wartungsintervalle und -aufwand beeinflusst. Hierbei sollte der variable Einlass die üblichen Wartungsintervalle des Triebwerks nicht verkürzen und auch den Wartungsaufwand nicht signifikant erhöhen. Das Sicherheitsrisiko infolge einer inkorrekten Wartung ist zu minimieren. Die Wartung sollte innerhalb einer kurzen Zeitspanne durch eine einzelne Person durchführbar sein und größtenteils aus Sichtproben bestehen.

Die zu erwartenden erhöhten Lebensweg- und Produktkosten variabler Einlässe müssen durch die Vorteile der variablen Geometrie kompensiert werden. Diese können im reduzierten Luftwiderstand und den dadurch verringerten Kraftstoffkosten sowie in einem erweiterten Betriebsbereich für Seitenwind und Anstellwinkel bestehen, vgl. Abschnitt 4.6.

\section{Entwicklungs- und Produktionskosten}

Die Entwicklungskosten des variablen Einlasssystems basieren auf den Erfahrungswerten mit den eingesetzten Technologien und Materialien im Bereich der Luftfahrt. Unzureichende Erfahrungswerte können vergrößerte Aufwände für Analysen und Tests erfordern. Daraus würden erhöhte Kosten und eine verlängerte Entwicklungsdauer resultieren.

Erhöhte Herstellungskosten können durch erforderliche innovative Materialien, spezielle Herstellungsverfahren, zusätzliche Qualitätskontrollen sowie einen 
erhöhten Montage- und Transportaufwand entstehen. Ein Teil dieser Kosten könnte durch recyclebare Komponenten kompensiert werden.

Das Einlasskonzept sollte zudem an verschiedene Triebwerksgrößen und Montagearten anpassbar sein. Weiterhin sollte das Konzept ein großes Potenzial für eine Patentierung aufweisen.

\subsubsection{Schnittstellenanalyse}

Ein variables Einlasssystem erfordert zahlreiche Schnittstellen mit seiner Umgebung, aber auch zwischen den Teilsystemen innerhalb des Einlasses. Unabhängig von den eingesetzten Teilsystemen zählen zu den Schnittstellen zwischen dem Einlass und seiner Umgebung stets

- seine mechanische Verbindung zur Triebwerksgondel,

- die umströmende Luft und mögliche Strömungsinteraktionen mit Triebwerksfan, -aufhängung, Tragflächen und Flugzeugrumpf,

- vom Fan ausgehende Schallwellen,

- anhaftende Feuchtigkeit mit der Möglichkeit der Eisbildung,

- Enteisungsfluide, die auf die Außenkontur gesprüht werden,

- Kollisionen mit Staub, Sand, Regen, Schnee, Hagel, Vulkanasche, angesaugten Kleinteilen, Vögeln und kleineren Drohnen,

- einschlagende Blitze,

- elektromagnetische Strahlung sowie

- Werkzeuge und Wartungspersonal, teilweise über Wartungszugänge.

Mögliche Teilsysteme eines variablen Einlasses sind

- ein Enteisungssystem,

- Lärmreduzierungsmaßnahmen,

- Messsonden zur Überwachung des Betriebs,

- Feuerschutzvorkehrungen sowie

- ein Variationsmechanismus, einschließlich umströmter Kontur, tragender Struktur und Aktorik.

Insbesondere für Enteisungssysteme, Lärmreduzierungsmaßnahmen und Variationsmechanismen existieren zahlreiche Umsetzungsmöglichkeiten, vgl. Abschnitt 2.3.2, 2.3.3, 2.4.2 und 2.4.3. In Abhängigkeit der gewählten Umsetzung der jeweiligen Teilsysteme variieren deren Komponenten und somit die 
resultierenden Schnittstellen untereinander. Schellin [313, S. 14-18] gibt einen detaillierten Überblick über mögliche resultierende Schnittstellen.

Ausgewählte Kombinationen von Teilsystemen weisen eine erhöhte Anfälligkeit für Feuerentstehung auf, weshalb Bereiche des Einlasses für diese Fälle als Feuerzonen auszuweisen sind. Diese Klassifizierung erfordert konstruktive Vorkehrungen und zusätzliche Sicherheitsnachweise, die im nachfolgenden Abschnitt zur Musterzulassungsanalyse erläutert werden. Eine Feuergefahr besteht bei Kombinationen brennbarer Fluide mit möglichen Zündquellen und vorhandenem Sauerstoff. Brennbare Fluide sind beispielsweise Öle zum Betrieb hydraulischer Aktoren sowie Enteisungsfluide auf Alkoholbasis. Mögliche Zündquellen sind heiße Oberflächen, wie zum Beispiel die Zapfluftleitungen von Heißlufteisschutzsystemen, oder Zündfunken, die durch Fehler elektrischer Komponenten entstehen können.

\subsubsection{Identifikation erforderlicher Luftsicherheitsnachweise}

Aus den behördlichen Vorgaben der EASA für die Luftfahrtzulassung eines Baumusters gehen zahlreiche Anforderungen an variable Einlässe hervor. Der Nachweis über die Erfüllung dieser Anforderungen ist durch mindestens eines der in Tabelle 3.3 aufgeführten Nachweismittel zu erbringen. Die relevanten behördlichen Vorgaben und mögliche Nachweismittel für variable Einlässe wurden in einer ersten Musterzulassungsanalyse von Schellin [313, S. 44-48] identifiziert und anschließend iteriert.

Im Rahmen der Zulassung wird der Einlass als Bestandteil der Triebwerksgondel vorrangig dem Flugzeug und nicht dem Triebwerk zugeordnet [34, 25.1091]. Für strahlgetriebene Flugzeuge gelten die Bauvorschriften der CS-25 [34] für große Flugzeuge mit einer maximal zulässigen Abflugmasse von mindestens $5.700 \mathrm{~kg}[34,25.1]$. Zudem hat die Einlassgestaltung auch einen großen Einfluss auf die Funktionsweise des Triebwerks, wodurch ausgewählte Regularien für Flugtriebwerke nach CS-E (Engines) [287] zusätzlich in Betracht zu ziehen sind $[34,25.901]$.

Basierend auf der Funktionsweise herkömmlicher Einlässe sowie möglicher Technologien variabler Einlässe, vgl. Kapitel 2, wurden die Paragrafen der CS-25 hinsichtlich ihrer Relevanz für die Zulassung variabler Triebwerkseinlässe analysiert. Neuentwicklungen, zu denen auch der variable Einlass zählt, sind zudem umfangreicher zu testen [34, 25.601]. In der Folge wurden 99 Paragrafen und zugehörige Nachweismittel identifiziert, vgl. Tabelle A.4 in Anhang A.3. Von den identifizierten Paragrafen sind 14 jedoch nur im Zusammenhang mit Feuerschutz 
zu betrachten, falls das ausgewählte Einlasskonzept durch ungeeignete Kombinationen von Teilsystemen als ausgewiesene Feuerzone zu deklarieren ist, vgl. Abschnitt 4.1.2.

Zahlreiche Paragrafen der CS-25 behandeln die potenziell wirkenden mechanischen Belastungen und die zu erfüllenden Anforderungen unter verschiedenen Betriebsbedingungen. Diese Anforderungen sind vom Flugzeug sowie seinen Komponenten und somit auch von der tragenden Struktur des variablen Einlasssystems zu erfüllen. So werden erlaubte Folgen, Sicherheitsfaktoren und Nachweismittel für Grenz- und Extremlasten erläutert, vgl. CS-25.301-307 [34]. Für Ermüdungslasten erfolgt dies in CS-25.571 [34]. Weiterhin werden die Maximallasten bei diversen Flug- und Landemanövern definiert, vgl. CS-25.333/337/349/351/371 bzw. CS-25.473/479/481/561 [34]. Darüber hinaus werden Anforderungen an die eingesetzten Materialien [34, 25.603/613], die Herstellungsverfahren [34, 25.605], die Montage [34, 25.607] sowie die Inspektion und die Wartung [34, 25.611] gestellt.

Das Flugzeug muss bei allen Betriebsbedingungen einfach und sicher zu steuern sein [34, 25.143]. Hierzu zählen beispielsweise starker Seitenwind [34, 25.237], Böen und Turbulenzen [34, 25.341] sowie Vereisungsbedingungen [34, 25.1093/1419/1420]. Weiterhin werden die Anforderungen bezüglich Blitzschlag [34, 25.581], Erdung elektrischer Komponenten [34, 25.899] und Vogelschlag [34, 25.631] beschrieben. Zusätzlich ist Schutz gegen weitere Wettereinflüsse, Korrosion und Abrieb erforderlich [34, 25.609]. Auf die von Vulkanasche ausgehende Gefahr wird gesondert eingegangen [34, 25.1593].

CS-25.901 [34] beschreibt explizit die Anforderungen an die Triebwerksinstallation und erfordert beispielsweise die Dokumentation von Schnittstellen gemäß CS-E20 [287]. Der Einlass muss im Zusammenspiel mit Kerntriebwerk und Düse unter allen Betriebsbedingungen seine Funktion erfüllen [34, 25.941]. Diese Funktion wird in CS-25.1091 [34] als Versorgung des Triebwerks mit der geforderten Luftmenge bei allen Betriebsbedingungen definiert. Gemäß CS-25.939 [34] darf der Einlass im Triebwerk keine Vibrationen als Folge ungleichförmiger Anströmbedingungen erzeugen. CS-25.1103 [34] stellt ausgewählte Ereignisse und zugehörige Belastungen heraus, denen der Einlass strukturell standhalten können muss. Dazu zählen

- Widerstandsfähigkeit gegen Pumplasten,

- die Gewährleistung der Sicherheit beim Bersten einer Zapfluftleitung eines Heißlufteisschutzsystems sowie

- Feuerresistenz und ein Feuerlöschsystem, falls der Einlass als Feuerzone ausgewiesen ist [34, 25.1103]. 
Die Einlasshülle muss aerodynamischen und systeminternen Lasten standhalten [34, 25.1193]. Auch Beschädigungen des Einlasses durch angesaugte Fremdkörper, Regen, Hagel und Vögel sollten vermieden werden [287, E790/800]. Weiterhin müssen Mittel zur Drainage und Ventilation gemäß CS-25.1187 [34] installiert sein. Zudem ist die Einlasshülle in jedem Fall feuerresistent umzusetzen [34, 25.1193], sodass sie einer Feuereinwirkung mindestens fünf Minuten widersteht [34, AMC 25.869]. Bei einer Klassifizierung des Einlasses als Feuerzone wird hingegen Feuerfestigkeit gefordert, die die Zeitspanne von 15 Minuten umfasst [34, AMC 25.869]. Die Thematik des Feuerschutzes wird in den Paragrafen CS-25.863/869 [34] sowie CS-25.1183-1207 [34] detailliert erläutert.

Zudem existieren Richtlinien für die Gestaltung, die Installation [34, 25.1353] und den Schutz elektrischer Systeme [34, 25.1357/1363], beispielsweise vor Blitzschlag [34, 25.1316] und Strahlungsfeldern [34, 25.1317]. Die Anforderungen an elektrische Leitungen und Verbindungen werden separat beschrieben, vgl. CS25.1701-1733 [34]. Richtlinien für die Gestaltung hydraulischer Systeme sind in CS-25.1435 [34] angegeben. Analog dazu gehen diese für pneumatische Systeme aus CS-25.1436 hervor [34].

An einigen Stellen verweist die CS-25 auf Regularien für Flugtriebwerke nach CS-E [287]. Die Anforderungen der CS-E für die Gestaltung und Zulassung eines variablen Systems im Bereich der Triebwerksgondel wurden von Grasselt [54, S. 95-101] am Beispiel variabler Düsen und Schubumkehrer untersucht. Diese Untersuchung hebt hervor, dass verstellbare Systeme in ihren ungünstigsten Einstellungen getestet [54, S. 96] und bei einem Funktionsausfall in einer sicheren Stellung fixiert werden sollten [54, S. 96].

Als Hauptresultat geht aus der Musterzulassungsanalyse hervor, dass die Kombination von Zündquelle und entflammbaren Medium innerhalb des Einlasses zu vermeiden ist. Somit kann die Klassifizierung als ausgewiesene Feuerzone umgangen und der Zulassungsaufwand des variablen Systems reduziert werden. Abgesehen von den in Abschnitt 1.1 erläuterten behördlichen Restriktionen bezüglich des Überschallknalls [41] wurden zwischen Unter- und Überschallanwendungen keine Unterschiede für die mögliche Zulassung variabler Einlässe identifiziert. Die Anforderungen, die aus den identifizierten Paragrafen und den zugehörigen AMCs hervorgehen, wurden in der Anforderungsliste in Tabelle A.3 in Anhang A.2 ergänzt. Abschließend wird eine weitere Iteration der Musterzulassungsanalyse nach Erreichen von Reifegrad TRL 3 empfohlen, da dann alle wesentlichen Konzeptbestandteile bekannt sind und somit berücksichtigt werden können. 


\subsubsection{Identifikation und Wichtung von Kriterien}

Nachfolgend werden die Ausschlusskriterien für die Vorauswahl der Konzepte erläutert. Darauf aufbauend erfolgt die Vorstellung der Auswahl und der Wichtung der Bewertungskriterien für die spätere gewichtete Punktbewertung von Lösungsvorschlägen.

\section{Ermitteln der Ausschlusskriterien}

Aus der Anforderungsliste in Tabelle A.3 wurden Ausschlusskriterien für die erste Bewertung der Konzeptideen hergeleitet. Diese Kriterien müssen von einem Einlasskonzept in jedem Fall bereits erfüllt werden oder perspektivisch erfüllbar sein. Aufgrund der frühzeitigen Phase der Vorauswahl sind die Kriterien qualitativer Natur. Zu den Ausschlusskriterien zählen

- eine hohe Sicherheit,

- eine große Geometrievariation,

- eine geringe Beeinträchtigung der Strömung,

- eine geringe Komplexität und

- eine gute Integrierbarkeit.

Die potenziellen Konzepte müssen perspektivisch bei minimaler Masse die Sicherheit gegen strukturelles Versagen im Fall eines Vogelschlags gewährleisten können. Zudem sollte das Risiko der Feuerentstehung durch Vermeidung einer ausgewiesenen Feuerzone minimiert werden.

Der Umfang und die Genauigkeit der ermöglichten Geometrievariation bestimmen das Einsparpotenzial eines Konzeptes. Zudem sollte die Variation in einer angemessenen Stellzeit erfolgen können. Weiterhin muss sichergestellt sein, dass die eingestellte Kontur erhalten bleibt.

Die störungsfreie Umströmung der Einlasskontur hat ebenfalls einen großen Einfluss auf die aerodynamischen Kennwerte des Einlasskonzepts, vgl. Abschnitt 2.3.1. Darüber hinaus hat sie auch einen signifikanten Einfluss auf die Lärmerzeugung durch den Einlass.

Die Komplexität des variablen Einlasssystems wird bestimmt durch die Anzahl der verwendeten Komponenten und Wirkmechanismen. Sie beeinflusst direkt die Ausfallwahrscheinlichkeit sowie den Wartungs- und den Montageaufwand des Konzepts.

Die Integrierbarkeit beschreibt, ob und wie gut die erforderlichen Teilsysteme des variablen Einlasses im Bauraum des untersuchten Konzepts unterzubringen 
sind. $\mathrm{Zu}$ diesen Teilsystemen zählen der Variationsmechanismus, ein Eisschutzsystem und eine Lärmreduzierungsmaßnahme.

\section{Ermitteln und Wichten der Bewertungskriterien}

Die Durchführung der gewichteten Punktbewertung von Lösungsvarianten während der Vorentwurfsphase erfordert gewichtete Kriterien. Für Lösungsalternativen bezüglich variabler Einlässe wurden die wichtigsten Bewertungskriterien aus dem Anforderungsdokument abgeleitet, vgl. Abschnitt 4.1.1. Diese Kriterien sind:

- die aerodynamische Funktionalität,

- die Integrierbarkeit,

- die Masse und die Widerstandsfähigkeit gegen Beanspruchungen,

- die Sicherheit,

- die Zuverlässigkeit und die Lebenswegkosten sowie

- die Entwicklungs- und die Produktionskosten.

Durch die eindeutige Zuordnung der Anforderungen aus Tabelle A.3 zu diesen Kategorien ist die thematische Überschneidung der einzelnen Bewertungskriterien weitestgehend minimiert worden. Dennoch verbleiben gewisse kausale Verkettungen zwischen Anforderungen, beispielsweise der Einfluss von Leichtbaumaterialien auf die Produktionskosten, vgl. Abschnitt 3.2.1.

Die Gewichtung der genannten Kriterien basiert auf dem paarweisen Vergleich mit einem Wertebereich von 0 bis 2, vgl. Tabelle 4.1. Der Vergleich wurde durch mehrere Luftfahrtingenieure durchgeführt, um somit die bestmögliche Objektivität gewährleisten zu können. Darüber hinaus wurden die Ergebnisse, soweit möglich, mit der Fachliteratur abgeglichen, vgl. Sadraey [284, S. 71].

Aus dem paarweisen Vergleich gehen die relativen Gewichtungsfaktoren $w_{i}$ der jeweiligen Kriterien hervor. Diese Ergebnisse offenbaren, dass die Gewährleistung der Sicherheit und bestmögliche aerodynamische Eigenschaften die wichtigsten Kriterien für Lösungsalternativen im Bereich variabler Einlässe sind, vgl. Abbildung 4.2.

\subsection{Funktionsanalyse}

Um den identifizierten Anforderungen gerecht zu werden, muss ein variabler Einlass mehrere Funktionen erfüllen können. Die identifizierten Funktionen werden nachfolgend erläutert und in den Gesamtzusammenhang des Flugzeugs 
Tabelle 4.1 Gewichtung der Kriterien durch paarweisen Vergleich

\begin{tabular}{l|l|l|l|l|l|l|l}
\hline Kriterium & A & I & W & S & R & D & $\begin{array}{l}\text { Relatives } \\
\text { Gewicht } \boldsymbol{w}_{\boldsymbol{i}}\end{array}$ \\
\hline $\begin{array}{l}\text { Aerodynamische } \\
\text { Funktionalität (A) }\end{array}$ & - & 2 & 2 & 1 & 1 & 2 & 0,267 \\
\hline Integrierbarkeit (I) & 0 & - & 1 & 0 & 0 & 1 & 0,067 \\
\hline $\begin{array}{l}\text { Masse und } \\
\text { Beanspruchungswiderstand } \\
(\mathbf{W})\end{array}$ & 0 & 1 & - & 0 & 1 & 2 & 0,133 \\
\hline Sicherheit (S) & 1 & 2 & 2 & - & 1 & 2 & 0,267 \\
\hline $\begin{array}{l}\text { Zuverlässigkeit und } \\
\text { Lebenswegkosten (R) }\end{array}$ & 1 & 2 & 1 & 1 & - & 1 & 0,200 \\
\hline $\begin{array}{l}\text { Entwicklungs- und } \\
\text { Produktionskosten (D) }\end{array}$ & 0 & 1 & 0 & 0 & 1 & - & 0,067 \\
\hline
\end{tabular}

Zellwerte: Reihenkriterium ist im Vergleich zum Spaltenkriterium wichtiger (2), gleich wichtig (1), sekundär (0)

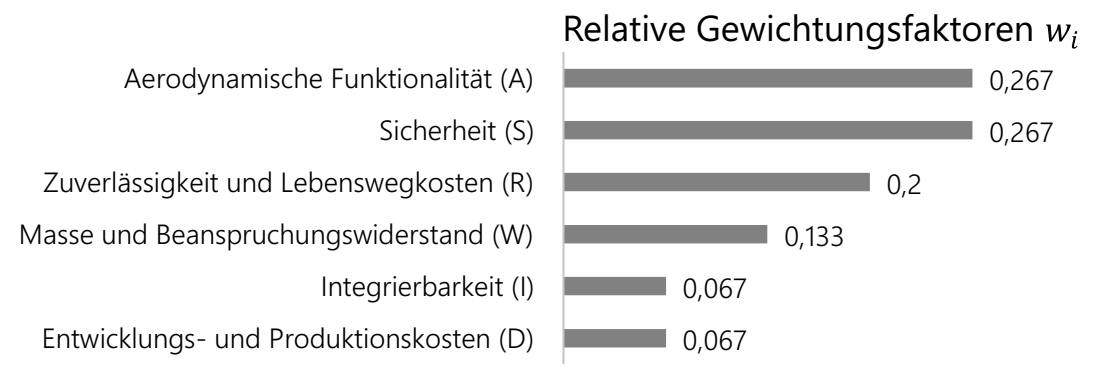

Abbildung 4.2 Gewichtete Bewertungskriterien

eingeordnet. Anschließend werden die Zusammenfassung zu Funktionsstrukturen, die Analyse möglicher Fehlerfolgen sowie mögliche Wirkmechanismen zum Umsetzen der jeweiligen Funktionen vorgestellt. 


\subsubsection{Funktionsidentifikation}

Die Hauptfunktion des Einlasses besteht darin, die Luftzufuhr zum Triebwerk zu gewährleisten. Dadurch wiederum kann das Triebwerk seine Hauptfunktion im Flugzeug erfüllen, nämlich die Erzeugung einer Schubkraft. Diese Schubkraft ermöglicht in Kombination mit der Umströmung der Tragflächen die Überwindung der Schwerkraft und somit das Fliegen.

Theoretisch ist für die Erfüllung der Hauptfunktion des Einlasses lediglich eine Eingangsströmung erforderlich. Diese wird basierend auf der Kontur des Einlasses in eine Ausgangsströmung umgewandelt. Bei der Umwandlung besteht eine weitere Funktion des Einlasses darin, den auftretenden Luftwiderstand zu minimieren.

In der Realität treten bei der genannten Umwandlung zusätzlich Störungen auf. Mögliche Störungsformen sind Böen, Regen, Eisbildung, Fremdkörper-, Hagel-, Vogel- und Blitzeinschläge. Durch diese Einschläge treten weiterhin Belastungen auf, die von der Einlassstruktur aufgenommen werden müssen. Um einer möglichen Eisbildung vorzubeugen, besteht zusätzlich eine Funktion des Einlasses in der Verhinderung von Eisansammlungen. Hierfür kann ein Eisschutzsystem im Einlass integriert werden, vgl. Abschnitt 2.3.2. Dieses erfordert wiederum Enteisungsenergie, die dem Einlass zugeführt werden muss. Eine weitere Funktion des Einlasses besteht in der Reduktion der Fanlärmemissionen, vgl. Abschnitt 2.3.3. Darüber hinaus kann vom Einlass die Funktion der Erfassung von Umgebungsdaten, wie Druck und Temperatur vor dem Fan, gefordert sein.

Die Erfüllung der geforderten Funktionen zur Gewährleistung der Luftzufuhr und der Minimierung des Luftwiderstands kann durch einen variablen Einlass verbessert werden. Dieser erfordert, zusätzlich zu den genannten Funktionen, das Variieren der umströmten Kontur, vgl. Abbildung 4.3. Ein solcher Variationsmechanismus benötigt als zusätzliche Eingangsgrößen ein Stellsignal und Hilfsenergie, vgl. Abschnitt 2.4.3. Weiterhin müssen potenzielle Störungen des Variationsmechanismus, beispielsweise durch elektromagnetische Felder und die zuvor genannten Einflüsse, beachtet werden.

\subsubsection{Funktionsstrukturanalyse}

Die Zusammenhänge zwischen den im vorherigen Kapitel identifizierten Funktionen werden nachfolgend durch Funktionsstrukturbäume veranschaulicht. Diese wurden auf den Hierarchie-Ebenen des Flugzeugs, des Einlasses und des Stellsystems erstellt. 


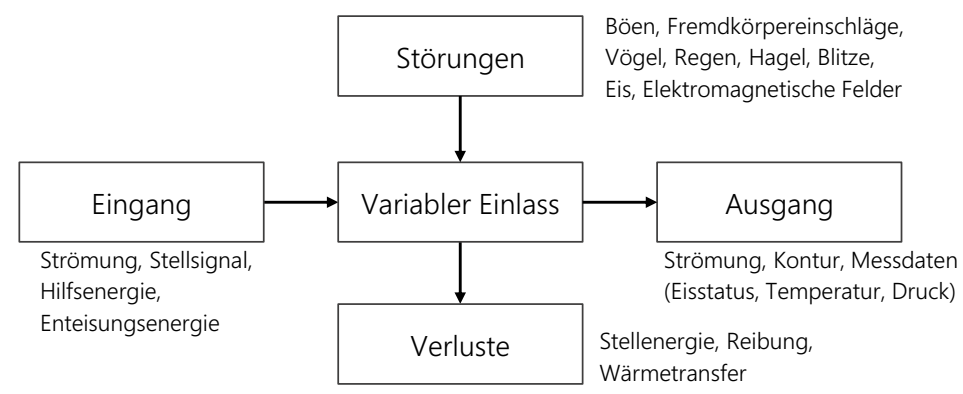

Abbildung 4.3 Funktion eines variablen Einlasses

\section{Flugzeugebene}

Eine der Hauptfunktionen des Flugzeuges ist das Steuern des Schubs [32], [53], vgl. Abbildung 4.4. Diese Funktion kann in die Erzeugung, das Anpassen, das Sicherstellen und das Bestimmen des Schubs unterteilt werden. Das Einlasssystem beeinflusst durch seine Funktion im Triebwerk die Umsetzung aller dieser Funktionen, vorrangig durch die Sicherstellung der Luftzufuhr. Darüber hinaus kann der Einlass Einfluss auf andere Hauptfunktionen des Flugzeuges, wie dessen Steuerung oder die Kabinenbelüftung, nehmen. Dies ist insbesondere bei einer Fehlfunktion des Einlasses der Fall.

\section{Einlassebene}

Die Hauptfunktionen auf der Detailebene des Einlasses bestehen darin

- die Luftzufuhr zum Triebwerk bei allen Betriebszuständen zu gewährleisten,

- den Luftwiderstand bei der Umströmung der Gondel zu minimieren,

- die wirkenden Belastungen aufzunehmen,

- den von Fan und Verdichter ausgehenden Lärm zu reduzieren sowie

- die Bildung von Eisansammlungen und deren Folgen zu verhindern.

Diese Hauptfunktionen lassen sich zudem in Teilfunktionen aufgliedern. So ist die Gewährleistung der Luftzufuhr unterteilbar in das Einstellen der Fananströmgeschwindigkeit, das Vermeiden von Strömungsablösungen und das Gewährleisten einer hohen Gleichförmigkeit, vgl. Abbildung 4.5. Die kombinierte Umsetzung dieser Funktionen für alle Betriebsbedingungen kann durch variable Einlasskonturen erleichtert werden. 


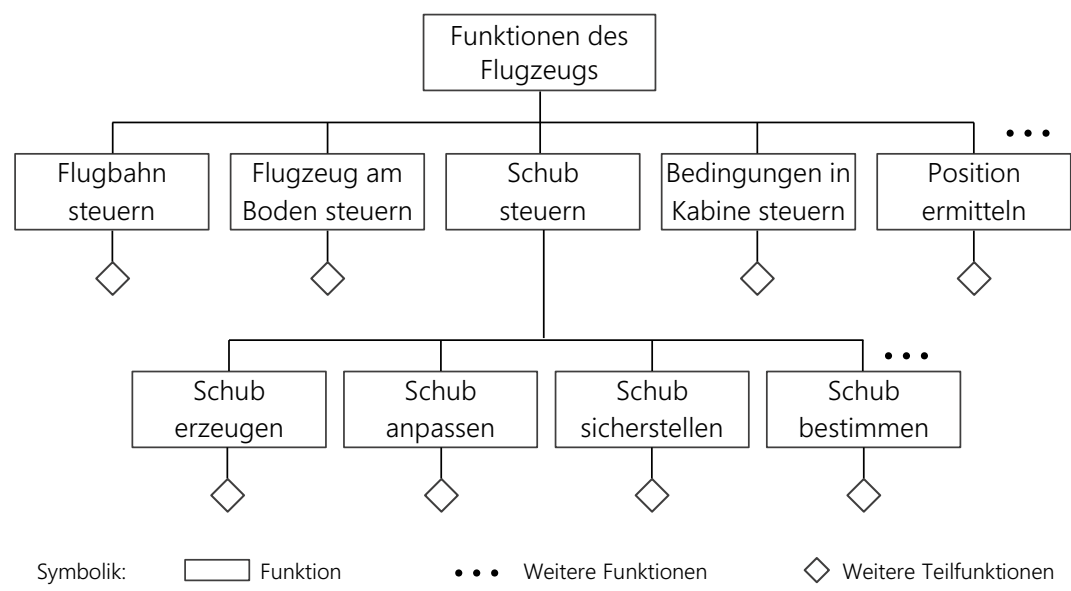

Abbildung 4.4 Funktionsstruktur des Flugzeugs

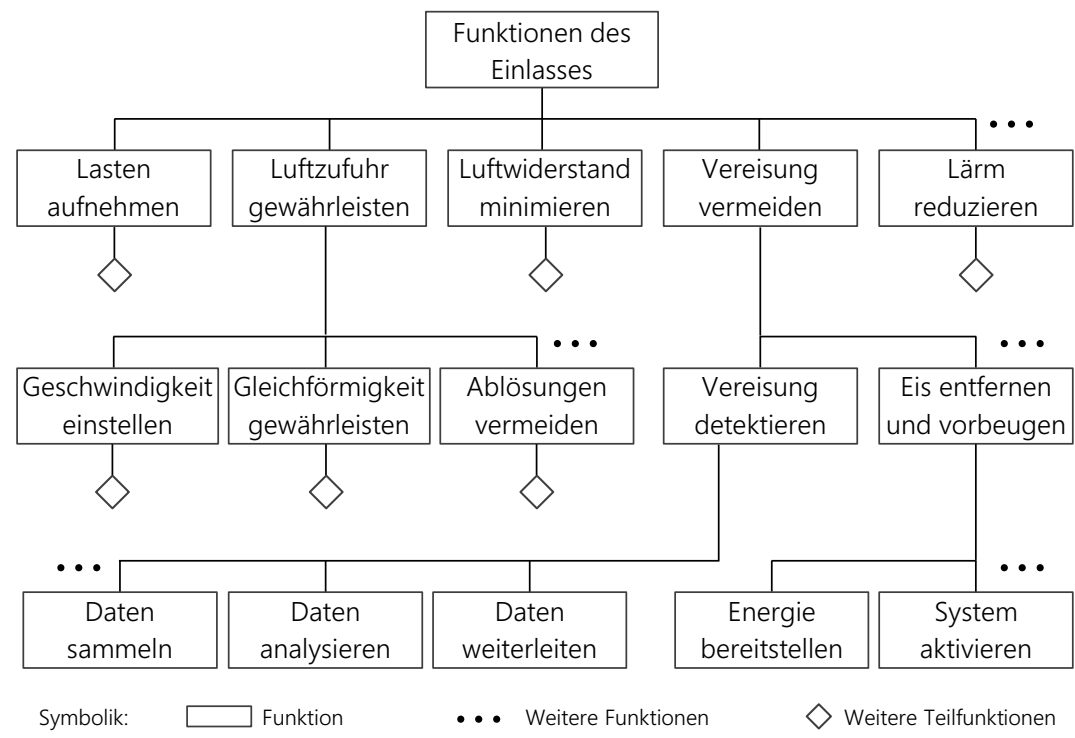

Abbildung 4.5 Funktionsstruktur des Einlasses 
Darüber hinaus offenbart die Aufgliederung der Funktion zur Vermeidung von Vereisung untergeordnete Teilfunktionen, wie die Bereitstellung von Energie, die Aktivierung eines Systems sowie die Erfassung und Verarbeitung von Daten. Diese Teilfunktionen sind ebenfalls für einen positionsgeregelten Aktor erforderlich, vgl. Abschnitt 2.4.3. Folglich besteht hier Potenzial für die Nutzung von Synergieeffekten zwischen dem üblicherweise vorhandenen Eisschutzsystem und einem möglichen Variationsmechanismus.

\section{Stellsystemebene}

Neben der angesprochenen Bereitstellung von Energie und der Regelung des Systems hat ein mögliches Stellsystem weitere Funktionen zu erfüllen. Dies betrifft insbesondere die Variation der umströmten Kontur, aber auch die Gewährleistung, dass die eingestellte Kontur sich nicht ungewünscht verändert, vgl. Abbildung 4.6. Bei der Verwendung eines Stellsystems mit mehreren Aktoren ist eine Synchronisation dieser erforderlich. In diesem Fall sind für die Gewährleistung der Geometrie zusätzlich die Teilfunktionen „Synchronisation sicherstellen“ und „Aktorausfall kompensieren“ umzusetzen.

Im Rahmen der Sicherheitsanalysen ist festzustellen, inwieweit die Teilfunktion der Gewährleistung der Geometrie „Teilausfall kompensieren“ umzusetzen ist. Die Umsetzung dieser Funktion hängt von den möglichen Folgen eines Funktionsausfalls des Stellsystems ab. Diese Funktionsausfälle sind Gegenstand der nachfolgenden Gefährdungsanalyse und der späteren Fehlerbaumanalyse.

Weiterhin ist der Einfluss der Variabilität auf die verbleibenden Einlassfunktionen zu untersuchen. Falls der Einlass in Abhängigkeit des gewählten Konzepts über keine starre, umlaufende Struktur verfügt, ist die konstruktive Umsetzung zur Erfüllung der Belastungsaufnahme anzupassen. Zudem kann die Variabilität durch einen verringerten oder formvariablen Bauraum Einfluss auf die Integration und die Funktionsweise herkömmlicher Lärmreduzierungsmaßnahmen nehmen. Umgekehrt darf die Variabilität nicht durch die Lösungen für die anderen Einlassfunktionen beeinträchtigt werden. Beispielsweise könnte die erzeugte Wärme einiger Eisschutzsysteme die Funktionsfähigkeit und die Lebensdauer möglicher verformbarer Oberflächenmaterialien beeinträchtigen. Im Rahmen der Zonensicherheitsanalyse werden diese Wechselwirkungen detailliert vorgestellt, vgl. Abschnitt 4.4.1. 


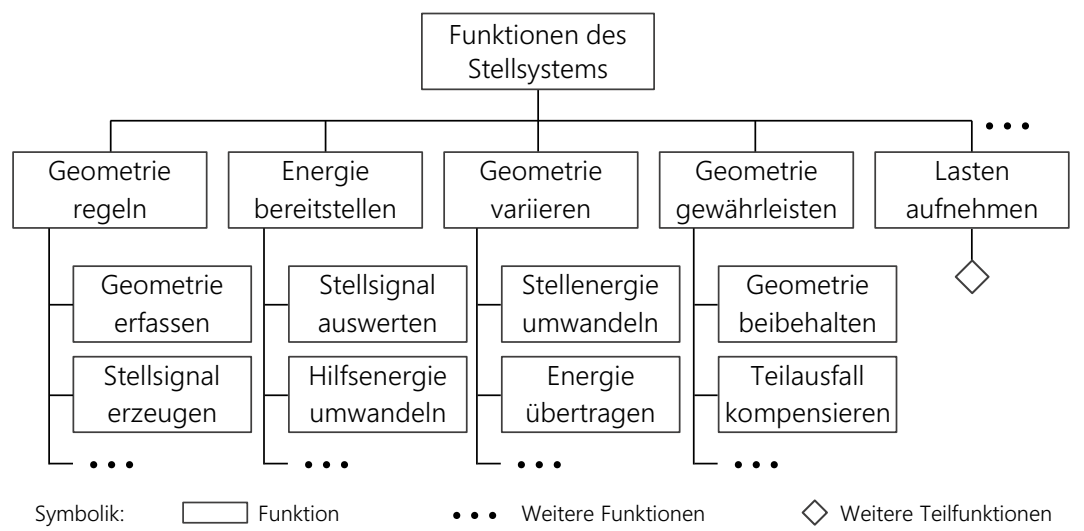

Abbildung 4.6 Funktionsstruktur des Stellsystems

\subsubsection{Gefährdungsanalyse}

Die systematische Analyse und Bewertung möglicher Fehler bei der Erfüllung von Systemfunktionen war Gegenstand der nachfolgend vorgestellten Gefährdungsanalyse (FHA). Die zuvor erarbeiteten Funktionsstrukturbäume dienten als Grundlage der FHA. So können Funktionsbeeinträchtigungen im Bereich des Einlasses auf verschiedene Weisen sicherheitskritische Auswirkungen auf Flugzeugebene haben. Möglichkeiten hierfür sind Fehlerereignisse, die bezüglich der Funktionen zum Aufnehmen von Lasten, Vermeiden von Vereisung und Gewährleisten der Luftzufuhr auftreten.

Durch eine unzureichende Luftzufuhr kann das Triebwerk nicht den geforderten Schub erzeugen. Dies kann in Abhängigkeit des Flugzustandes und der Anzahl der betroffenen Triebwerke sicherheitskritische Folgen nach sich ziehen. Die Gewährleistung der Luftzufuhr kann bei Verwendung variabler Einlässe durch Fehlfunktionen des Stellmechanismus gefährdet werden. Mögliche Fehlfunktionen des Stellmechanismus können dahingehend unterschieden werden,

- inwiefern sich das Fehlerereignis auf die eingestellte Geometrie auswirkt,

- wie stark die Funktionsbeeinträchtigung ausfällt oder

- ob das Fehlerereignis korrekt detektiert wird. 
Im Folgenden werden Fehler des Stellsystems, die zur Folge haben, dass eine unerwünschte Geometrie eingestellt wird, zusammengefasst betrachtet. Eine solche Geometrie, die nicht zum vorherrschenden Flugzustand passt, kann zur Folge haben, dass Strömungsablösungen bis hin zum Schubverlust durch Verdichterpumpen auftreten können. Dabei ist zu unterscheiden, ob nur der variable Einlass eines einzelnen Triebwerks betroffen ist oder ob bei mehreren Triebwerken eine unerwünschte Geometrie eingestellt wird. Darüber hinaus hat die Flugphase, während welcher die unerwünschte Geometrie eingestellt ist, einen entscheidenden Einfluss auf die Schwere der möglichen Folgen. Die Schwere der Folgen einer unerwünschten Geometrie kann nach CS-25.1309 [34] bewertet und diesen Folgen erlaubte Eintrittswahrscheinlichkeiten zugeordnet werden, vgl. Tabelle 3.1.

Für den Fall, dass nur ein Triebwerk betroffen ist und dieses somit potenziell keinen Schub erzeugt (One Engine Inoperative), sind die Folgen als unbedeutend (Minor) zu klassifizieren, vgl. Tabelle 4.2. Die möglichen Folgen bestehen hierbei in einer geringen Reduzierung der Flugzeugfunktionsfähigkeit sowie einer geringen Zunahme der Arbeitsbelastung der Besatzung. Dies ist damit zu begründen, dass der fehlende Schub durch die verbleibenden Triebwerke zu kompensieren ist und auch die Steuerung des Flugzeugs weiterhin möglich sein muss [34, 25.147].

Fehler der Stellsysteme mehrerer Triebwerke können zu einem Schubverlust an mehr als einem Triebwerk führen. Dies kann beispielsweise während des Flugzeugstarts zu einem Startabbruch (Rejected Take-Off) führen. Ein Startabbruch ist nach CS-25 als gefährliches Ereignis mit einer erlaubten Eintrittswahrscheinlichkeit von weniger als $10^{-7}$ Ereignissen pro Flugstunde klassifiziert [34, 2-F-168 f.]. Der Nachweis dieser Eintrittswahrscheinlichkeit erfordert den Absicherungsgrad DAL B [53, S. 44]. Dieser kann die Integration zusätzlicher Sicherheitsmaßnahmen, beispielsweise in Form von Redundanz, erfordern [79, S. 212].

Ist die Funktion zur Aufnahme von Lasten fehlerbehaftet, können strukturelle Schäden bis hin zum Verlust des gesamten Einlasses auftreten, vgl. Air France Flug AF-66 [319], [320]. Strukturelle Schäden können den bereitstellbaren Schub und den erzeugten Luftwiderstand beeinflussen. Weiterhin können verloren gegangene Komponenten zu Folgeschäden an anderen Bauteilen, wie beispielsweise dem Leitwerk, führen, diese beschädigen und somit die Kontrollierbarkeit des Flugzeuges gefährden, was katastrophale Folgen haben kann. Folglich muss der Verlust großer Komponenten des Einlasses die Eintrittswahrscheinlichkeit von $10^{-7}$ Ereignissen pro Flugstunde unterschreiten, was mit dem Absicherungsgrad DAL A nachzuweisen ist. Dieser Nachweis erfolgt im Rahmen der Konzeptdimensionierung, vgl. Abschnitt 4.5.2. 


\section{Tabelle 4.2}

Fehlerklassifizierung ,unerwünschte Einlassgeometrie" bei einem Triebwerk

\begin{tabular}{l|l|l}
\hline Flugphase & Klassifizierung & $\begin{array}{l}\text { Erlaubte } \\
\text { Ereignisse pro } \\
\text { Flugstunde }\end{array}$ \\
\hline $\begin{array}{l}\text { Triebwerksstart, } \\
\text { Leerlauf, Rollen }\end{array}$ & $\begin{array}{l}\text { kein } \\
\text { Sicherheitseffekt }\end{array}$ & $\begin{array}{l}\text { Keine } \\
\text { Anforderung }\end{array}$ \\
\hline $\begin{array}{l}\text { Flugzeugstart, } \\
\text { Steigflug }\end{array}$ & Unbedeutend & $<1$ E-03 \\
\hline Startabbruch & $\begin{array}{l}\text { kein } \\
\text { Sicherheitseffekt }\end{array}$ & $\begin{array}{l}\text { Keine } \\
\text { Anforderung }\end{array}$ \\
\hline $\begin{array}{l}\text { Reiseflug, } \\
\text { Sinkflug, } \\
\text { Landeanflug }\end{array}$ & kein \\
Sicherheitseffekt & $\begin{array}{l}\text { Keine } \\
\text { Anforderung }\end{array}$ \\
\hline Durchstarten & Unbedeutend & $<1$ E-03 \\
\hline Landung & kein & $\begin{array}{l}\text { Keine } \\
\text { Anforderung }\end{array}$ \\
\hline
\end{tabular}

Fehler beim Erfüllen der Funktion zur Vermeidung von Vereisung [34, AMC 25.21(g)] können in gefährlichen Ereignissen durch Eisansammlungen resultieren. Die Eisansammlungen können die Strömung negativ beeinflussen und im schlimmsten Fall zum Schubverlust führen. Weiterhin können abplatzende Eisansammlungen sicherheitskritische strukturelle Schäden am Fan verursachen. Darüber hinaus können Eisansammlungen die Funktionsfähigkeit des Variationsmechanismus eines variablen Einlasses einschränken. Zudem sind Wechselwirkungen zwischen dem Eisschutzsystem und dem Stellmechanismus, welche die jeweilige Funktionsfähigkeit verringern, zu vermeiden. Die Sicherheit des gewählten Eisschutzsystems ist in späteren Entwicklungsphasen gemäß CS-25 [34, AMC 25.1419] nachzuweisen.

\subsubsection{Identifikation von Lösungsprinzipen}

Nachfolgend wird die Identifikation von Wirkmechanismen bzw. Lösungsprinzipen für die umzusetzen Funktionen vorgestellt. Dies betrifft die Lösungsprinzipe für die Umsetzung von

- Vereisungsschutz,

- Lärmminderung,

- struktureller Lastaufnahme, 
- Geometrievariation,

- Aktorik und

- Konturerfassung.

Hierfür wurden Lösungsprinzipe aus der Natur, von erprobten Anwendungen sowie aus neuartigen Technologien in Betracht gezogen und in der nachfolgenden Konzeptphase zu Konzeptvarianten kombiniert.

\section{Lösungsprinzipe aus der Natur}

So wie sich die frühen Pioniere der Luftfahrt bereits am Flug der Vögel, Insekten und Fledermäuse orientierten, können auch relevante Lösungsprinzipe für variable Einlässe von der Natur abgeleitet werden [249, S. 2]. Diese bionischen Ansätze könnten beispielsweise für den Vereisungsschutz oder die Geometrievariation Anwendung finden.

Zahlreiche innovative Eisschutzsysteme nutzen eisabweisende Beschichtungen [123], [124], [125]. Diese Beschichtungen verwenden Mikro- und Nanostrukturen, die das Anhaften von Wasser, Staub und Sand auf der Oberfläche verhindern. Dieses als Lotus-Effekt bekannte Prinzip findet man in der Natur bei verschiedenen Blättern von Pflanzen, aber auch an den Flügeln von Insekten und der Haut von Wüstenreptilien, wie dem Apothekerskink [321].

Zur Umsetzung einer Konturvariation sind in der Natur elastische Häute weit verbreitet. Beispielsweise nutzen Seiga Antilopen in den Steppen der Mongolei eine große aufblasbare Nase, um die Luft beim Einatmen zu filtern und zu erwärmen sowie zur Erzeugung von Lauten [322], vgl. Abbildung 4.7. Dabei hat die Nase, ähnlich dem Einlass, die Grundform eines Hohlzylinders. Bei den männlichen Exemplaren der Klappmützenrobben befindet sich die Nase in Form eines aufblasbaren Balgs auf der Stirn. Auch verfügen Rentiere über einen stark aufblasbaren Kehlkopf [323]. Ebenso sind die Körper der meisten Quallen und einiger Oktopodenarten stark verformbar.

Die Krallen von Katzen sind nicht elastisch verformbar, jedoch überwiegend ausfahrbar. Ein durch Muskeln, Sehnen und Knochen gesteuerter Mechanismus erlaubt den Katzen bei Bedarf den Einsatz der Krallen, während diese beim Laufen vor Abnutzung geschützt sind [324].

\section{Lösungsprinzipe aus dem Bereich der Technik}

Für den Vereisungsschutz können die in Abschnitt 2.3.2 vorgestellten erprobten Anwendungen, aber auch innovative Lösungsansätze, wie das Nutzen von Formgedächtnismaterialien, herangezogen werden. 


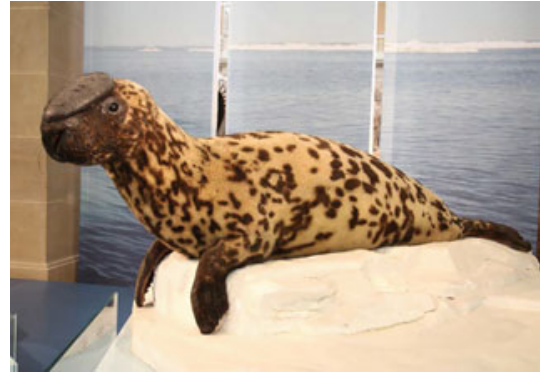

Nase der Klappmützenrobbe

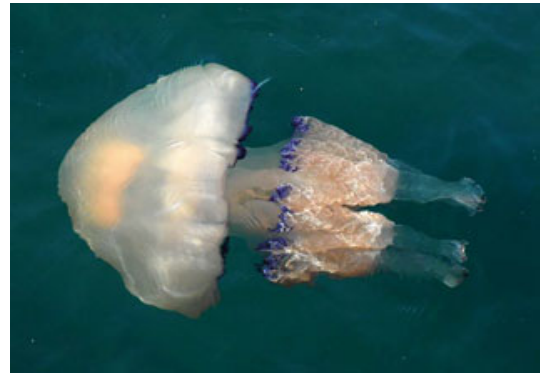

Quallenkörper

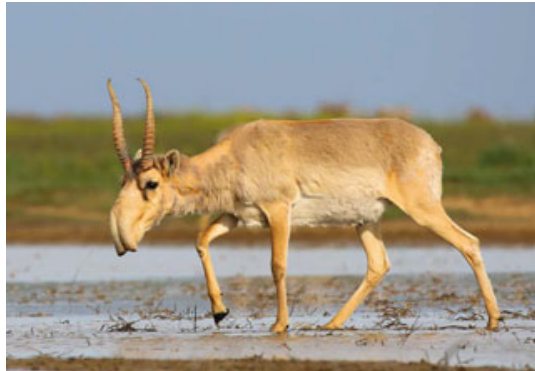

Nase der Seiga Antilope

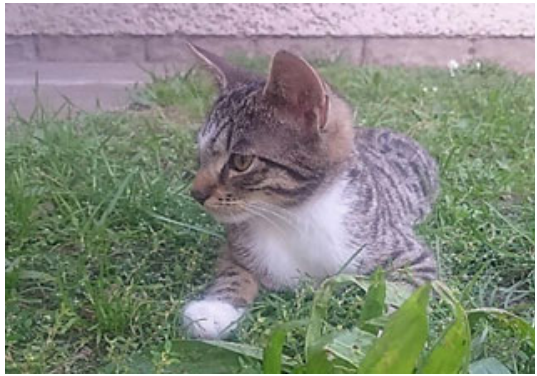

Einziehbare Katzenkrallen

Abbildung 4.7 Bionische Lösungsansätze für Variabilität [A48]

Im Bereich der Lärmminderung werden vorrangig Akustikauskleidungen als etablierte Lösung betrachtet, vgl. Abschnitt 2.3.3. Sollte das ausgewählte Konzept jedoch eine formvariable Außenhülle erfordern, können für die Lärmminderung Anpassungen erforderlich werden. In diesem Fall sollten Akustikauskleidungen mit flexiblen Wänden, vgl. Mischke et al. [325], [326], [327], oder die Schallauslöschung durch Gegenschall detailliert untersucht werden.

Zur Aufnahme struktureller Lasten können monolithische Strukturen mit eingeschlossenen Versteifungsringen entsprechend des Standes der Technik genutzt werden, vgl. Abschnitt 2.2.2. Darüber hinaus könnte die Lastaufnahme auch über die Spant-Stringer-Bauweise oder die Fachwerkbauweise erfolgen [77, S. 391-397].

Bei der Auswahl der Aktorik sind elektromechanische Aktoren zu bevorzugen, vgl. Abschnitt 2.4.3. Hydraulische, pneumatische und unkonventionelle Aktoren stellen mögliche Alternativen dar. Die von der Aktorik erzeugte Bewegung kann 
beispielsweise linear oder kreisförmig sowie in axialer oder radialer Richtung erfolgen. Die Erfassung der Kontur kann ein integraler Bestandteil der Aktorik sein, vgl. Abschnitt 2.4.3. Umgesetzt werden kann die Konturerfassung beispielsweise durch Drehwinkelgeber, Differentialtransformatoren zur Wegmessung oder optische Messverfahren.

Prinzipe, die für die Geometrievariation in Betracht gezogen werden können, sind das Verschieben starrer Segmente, das Verformen eines elastischen Oberflächenmaterials und die aerodynamische Grenzschichtbeeinflussung. Für die beiden letztgenannten Lösungsprinzipe kann die grundlegende Funktionsweise aus Abschnitt 2.4.2 entnommen werden. Für das Verschieben starrer Segmente werden nachfolgend die Prinzipe der Querschnitts-, Konturdicken-, Lippen- und Längenvariation vorgestellt.

\section{Querschnittsvariation}

Radiale Variationen eines Kreisdurchmessers erfordern bei Verwendung starrer Komponenten eine Segmentierung über den Umfang, um die Entstehung von Bauteilkollisionen zu vermeiden. Jedoch entstehen durch die Segmentierung bei der Querschnittserweiterung Spalte, vgl. Abbildung 4.8.

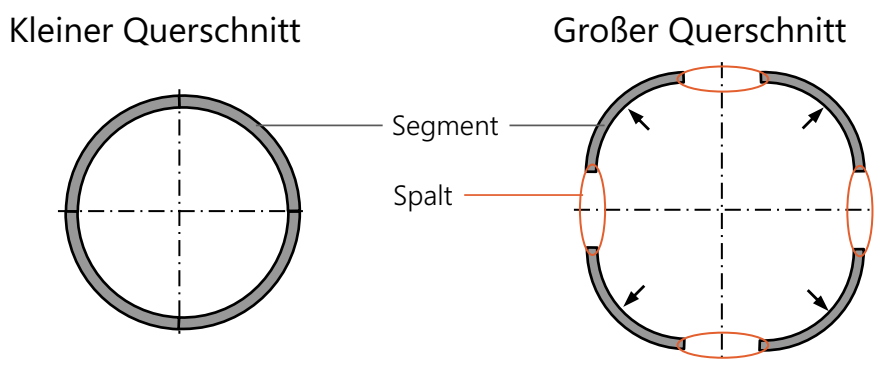

Abbildung 4.8 Segmentierung über den Umfang

Die Anzahl der Teilsegmente hat einen entscheidenden Einfluss auf die Güte der erzeugten Geometrie und die Zuverlässigkeit des Konzepts. Eine geringere Anzahl erzeugt eine Geometrie, die stärker von einem idealen Kreisquerschnitt abweicht und somit eine geringere Gleichförmigkeit der Fananströmung erzeugt. Gleichzeitig reduziert sie allerdings die Anzahl der erforderlichen Bauteile, was sich positiv auf die Komplexität und Zuverlässigkeit des Systems auswirken kann. 
Die über den Umfang der Kontur entstehenden Spalte zwischen den Segmenten sind abzudichten. Existierende Ansätze hierfür reichen von

- der Akzeptanz des Spalts [218] über

- starre Einfassungen zwischen den Segmenten [31], [214],

- Überlappungen benachbarter starrer Segmente [30], [158] bis hin zur

- Nutzung von elastischen Materialien zwischen den Segmenten [30].

Die beiden erstgenannten Varianten stellen aufgrund der schlechten aerodynamischen Eigenschaften keine zu erwägende Option dar.

Überlappungen benachbarter starrer Segmente zur Umfangsspaltabdichtung sind beispielsweise umsetzbar durch

- bewegliche Zwischensegmente [30],

- lineare Verlängerungen der Segmente oder

- Rotation der Segmente, vgl. Abbildung 4.9.

Bewegliche Zwischensegmente erhöhen die Anzahl der erforderlichen Komponenten im Vergleich zu den anderen Beispielen und haben somit einen verstärkten negativen Einfluss auf die Komplexität und die Zuverlässigkeit der Konstruktion. Zudem entstehen radiale Stufen auf der Außenseite der Geometrie. Auch bei der Verwendung rotierender Segmente entstehen Stufen auf der Außenseite der Geometrie, die einen negativen Strömungseinfluss haben können. Diese können zwar durch eine größere Anzahl an Teilsegmenten verringert, jedoch nicht vollständig vermieden werden. Lineare Verlängerungen der Segmente erzeugen kleinere radiale Stufen auf der Außenseite. Weiterhin ist die Reibung zwischen Verlängerungen benachbarter Segmente zu minimieren. Diese Variante vereint die besten geometrischen Eigenschaften mit einer einfachen konstruktiven Umsetzung.

Die Spaltabdeckung kann auch unter Verwendung dehnbarer Materialien zwischen den Segmenten erfolgen [30]. Diese können beispielsweise beidseitig oder einseitig fest eingespannt sein, vgl. Abbildung 4.10. Diese Varianten bieten eine aerodynamisch günstige Geometrie bei eingeschränkter Lebensdauer.

Aufgrund der genannten Vor- und Nachteile werden im weiteren Verlauf vorrangig lineare Verlängerungen zur Spaltabdichtung bei Verwendung starrer Segmente berücksichtigt. Die konstruktive Gestaltung der linearen Segmentverlängerungen wird in Abbildung 4.54 in Abschnitt 4.5 detailliert beschrieben. 
Bewegliches Zwischensegment

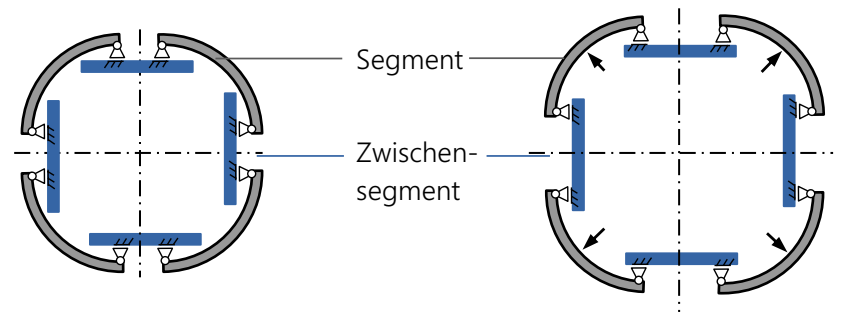

Lineare Segmentverlängerung

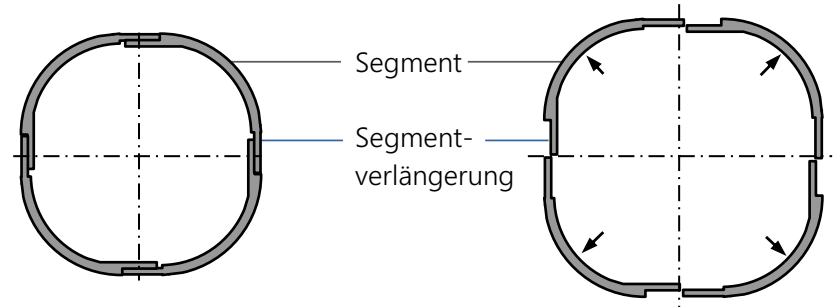

Rotierende Segmente
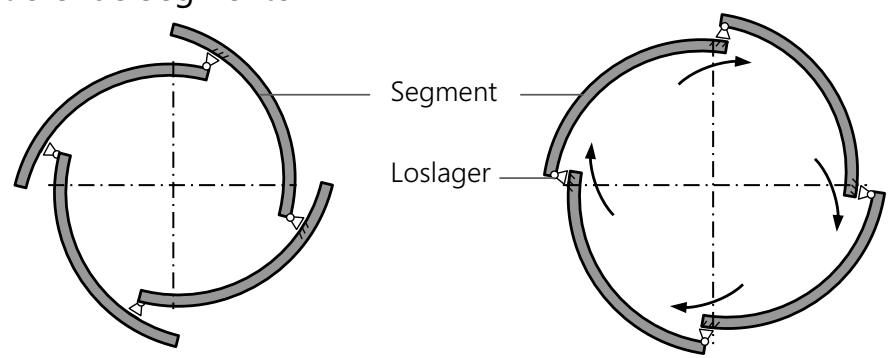

Abbildung 4.9 Querschnittsvariation mit starren Segmenten

\section{Konturdickenvariation}

Das Prinzip der Querschnittsvariation mit Hilfe linearer Segmentverlängerungen kann genutzt werden, um sowohl die Innen- als auch die Außenkontur des Einlasses zu variieren, vgl. Abbildung 4.11. Dadurch wird eine größere Variation der Profildicke sowie eine bessere Anpassung an die aerodynamischen Anforderungen ermöglicht. 


\section{Dehnbares Elastomer}

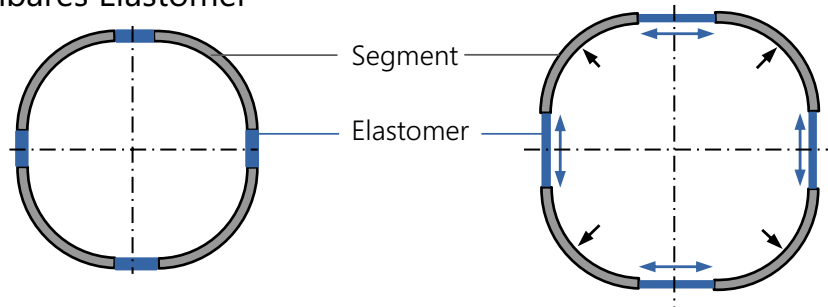

Verschiebbares Elastomer

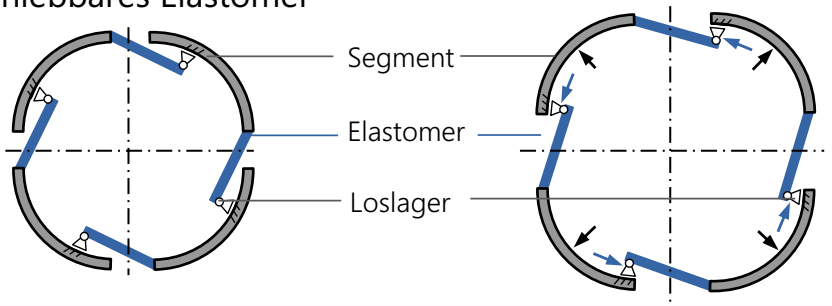

Abbildung 4.10 Querschnittsvariation mit Elastomeren

\section{Dicke Kontur}

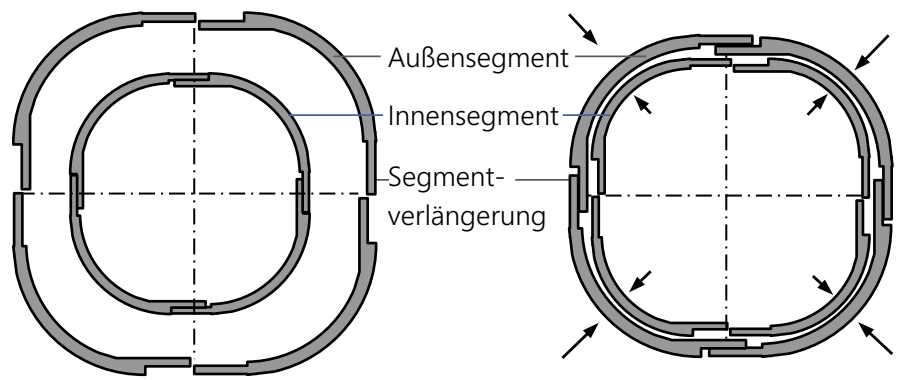

Abbildung 4.11 Konturdickenvariation durch verschiebbare starre Segmente

\section{Lippendickenvariation}

Bei der Verwendung starrer Segmente sind für die Variation der Lippendicke komplexe Mechaniken erforderlich. Eine Möglichkeit besteht darin, die Lippe axial und über den Umfang zu segmentieren. Die Dicke der segmentierten 
Lippe lässt sich anschließend durch Rotieren der Lippensegmente verändern. Auch das Verschieben sich radial überlappender Lippensegmente kann als eigenständige Variante genutzt werden. Eine weitere Möglichkeit besteht in der Verwendung rotierender Segmente, die mit einem geschlossenen Vorderkantenring verbunden sind. Bei dieser Variante ist jedoch die Festlegung auf genau einen Vorderkantendurchmesser erforderlich.

Bei allen genannten Varianten der Lippendickenvariation treten mindestens in einem Betriebszustand Stufen und Spalte im Bereich der Vorderkante auf, insbesondere bei der simultanen Variation des Vorderkantenquerschnitts. Der Spaltentstehung kann durch Abdichtungen entgegengewirkt werden. Bei einer Variation des Vorderkantenquerschnitts mittels starrer Abdichtelemente müssen diese über Kardan- oder Kugelgelenke mit den Segmenten verbunden werden, da hierbei eine Bewegung um zwei Achsen erforderlich wird [314, S. 87-90], [315, S. 105-106]. Wird hingegen eine elastische Abdichtung genutzt, unterliegt diese großen Beanspruchungen in mehrere Richtungen. Beide Varianten hätten einen negativen Effekt auf Komplexität und Lebensdauer. Die Umsetzungsmöglichkeiten der Lippenvariation und Abdichtung sowie deren Auswahl werden detailliert in Abschnitt 4.5.1 vorgestellt.

\section{Längenvariation}

Kleinere Variationen der Einlasslänge sind als Nebeneffekt bei der Verringerung der Konturwölbung erreichbar. Signifikante Längenvariationen des Einlasses erfordern hingegen stets das Ausfahren einander zuvor überlappender Komponenten gemäß des Teleskopprinzips. Diese Überlappung geht mit einer zusätzlich erforderlichen Masse und Komplexität einher.

\subsection{Konzeptphase}

Die Identifikation prinzipiell geeigneter Konzepte ist von großer Bedeutung für die weitere Konzeptstudie, da Fehler hierbei im weiteren Verlauf nicht oder nur unter erheblichem Aufwand behoben werden können [260, S. 341]. Erprobte Technologien bieten hierfür Sicherheit, laufen allerdings Gefahr, nicht das volle Potenzial einer Technologie auszuschöpfen [260, S. 342]. Bei innovativen Umsetzungen kann das Potenzial, auf Kosten eines erhöhten Entwicklungsrisikos, womöglich besser ausgeschöpft werden [260, S. 342].

Nachfolgend werden die intuitive und die methodische Erstellung der Konzeptvarianten beschrieben. Danach erfolgt die Vorstellung der erstellten Konzepte und deren Vorauswahl anhand der Bewertung ihrer prinzipiellen Eignung. 
Die prinzipiell geeigneten Konzepte wurden im Anschluss zu Konzeptgruppen zusammengefasst. Diese Konzeptgruppen werden beschrieben und die anschlieBende vorläufige Systemsicherheitsanalyse, basierend auf den zuvor identifizierten gefährlichen Funktionsausfällen, wird vorgestellt.

\subsubsection{Konzepterstellung}

\section{Intuitive Konzeptfindung}

$\mathrm{Zu}$ Beginn der Konzepterstellung wurden in vier Brainstorming-Sitzungen mit jeweils zwei bis acht Luftfahrtingenieuren erste Lösungsideen erarbeitet. Die jeweiligen Sitzungen wurden ergebnisoffen durchgeführt, um ein möglichst breit gefächertes Lösungsspektrum abzudecken.

Für das ergebnisoffene Generieren von Konzeptideen wurde den Sitzungsteilnehmern die zuvor definierte Aufgabenstellung dargelegt. Weiterhin wurden keine umzusetzenden Geometrien vorgegeben. Als Orientierung konnten bei Bedarf die Geometrien gemäß Abbildung 1.1 sowie Abbildung 4.1 genutzt werden. Dadurch wurde der Ergebnisraum bei der Ideenfindung maximiert. Diese Vorgehensweise resultierte in der Ermittlung zahlreicher verschiedener Lösungsideen, die am Ende dieses Teilkapitels vorgestellt werden.

\section{Methodische Konzeptfindung}

Zusätzliche Lösungsideen wurden mit der Methode des morphologischen Kastens erarbeitet. Hierbei wurden die zuvor identifizierten Funktionen und Lösungsprinzipe zusammengefasst, vgl. Tabelle 4.3. Durch Kombinieren der Lösungsprinzipe für die jeweiligen Funktionen konnten weitere Konzepte erstellt werden. Bei dieser Erstellung war zu berücksichtigen, dass einzelne Teillösungen nicht uneingeschränkt miteinander kombinierbar sind. Beispielsweise ist die Kombination eines Heißlufteisschutzsystems mit einem hydraulischen Aktor zu vermeiden, um die Feuergefahr innerhalb des Einlasses zu minimieren, vgl. Abschnitt 4.1.3.

\section{Identifizierte Konzepte}

Durch die beschriebene Herangehensweise wurden insgesamt 30 Konzepte für variable Einlässe identifiziert. Diese Konzepte sind in Tabelle 4.4 aufgelistet. Zusätzlich erfolgt die Angabe der variierbaren geometrischen Parameter:

- Einlassquerschnittsfläche (A),

- Vorderkantenrundung (R),

- Profillänge (L) und/oder

- Profildicke (T), 
Tabelle 4.3 Morphologischer Kasten für variable Einlässe

\begin{tabular}{l|l|l|l}
\hline Funktion & Lösungsansatz 1 & Lösungsansatz 2 & Lösungsansatz 3 \\
\hline Vereisungsschutz & Heißluft & Elektrothermisch & Elektromechanisch \\
\hline Lärmreduktion & $\begin{array}{l}\text { Herkömmliche } \\
\text { Akustik- } \\
\text { auskleidungen }\end{array}$ & Gegenschall & $\begin{array}{l}\text { Elastische Akustik- } \\
\text { auskleidungen }\end{array}$ \\
\hline Strukturelle Bauweise & Versteifungsringe & $\begin{array}{l}\text { Monolithische } \\
\text { Bauweise }\end{array}$ & Fachwerkbauweise \\
\hline $\begin{array}{l}\text { Variationsmechanis- } \\
\text { mus }\end{array}$ & $\begin{array}{l}\text { Verschiebung } \\
\text { starrer Segmente }\end{array}$ & $\begin{array}{l}\text { Elastische Oberflä- } \\
\text { chenverformung }\end{array}$ & $\begin{array}{l}\text { Aerodynamische } \\
\text { Grenzschichtbeein- } \\
\text { flussung }\end{array}$ \\
\hline Stellsystem & $\begin{array}{l}\text { Elektromechani- } \\
\text { sche } \\
\text { Aktoren }\end{array}$ & $\begin{array}{l}\text { Elektrohydraulische } \\
\text { Aktoren }\end{array}$ & $\begin{array}{l}\text { Unkonventionelle } \\
\text { Aktoren }\end{array}$ \\
\hline Konturerfassung & $\begin{array}{l}\text { Differentialtrans- } \\
\text { formatoren }\end{array}$ & Drehwinkelgeber & Optische Erfassung \\
\hline
\end{tabular}

und auf welchem Lösungsprinzip die Geometrievariation basiert:

- Verschieben/Rotieren fester Segmente einer geschlossenen Kontur (F),

- elastisches Verformen des Oberflächenmaterials (E),

- Bewegen von Vorkörpern/Klappen/Rampen/Konen (V) und/oder

- aerodynamisches Beeinflussen der Strömungsgrenzschicht (G).

Darüber hinaus können Anhang A.4 alle zugehörigen Konzeptskizzen entnommen werden. Eine Beschreibung der Funktionsweise eines Großteils dieser Konzepte ist in der Arbeit von Pauly [312, S. 27-80] vorzufinden.

Unter den identifizierten Konzeptideen ähneln einige bereits patentierten Ideen, vgl. Abschnitt 2.4.2. So findet das Kippen des Einlasses, vgl. Abbildung 2.28, Anwendung in Konzept 23, vgl. Abbildung A.23. Konzept 20 (Abbildung A.20) greift das radiale Verschieben eines Halbrings auf. Auch das Rotieren des Einlasses oder eines Anteils, vgl. Abbildung 2.29, spiegelt sich in den Konzepten 5 (Abbildung A.5), 6 (Abbildung A.6), 19 (Abbildung A.19) und 24 (Abbildung A.24) wider. Das Teleskop-Prinzip, vgl. Abbildung 2.30 links, ist in den Konzepten 16 (Abbildung A.16) und 21 (Abbildung A.21) wiederzufinden. Auch das Prinzip des axialen Ausfahrens von Lippe oder Profilen, vgl. Abbildung 2.30 mittig bzw. rechts, wird in Konzept 7 (Abbildung A.7) verwendet. Radial versetzte Strömungsprofile, vgl. Abbildung 2.31 links, sind in variabler Form in Konzept 


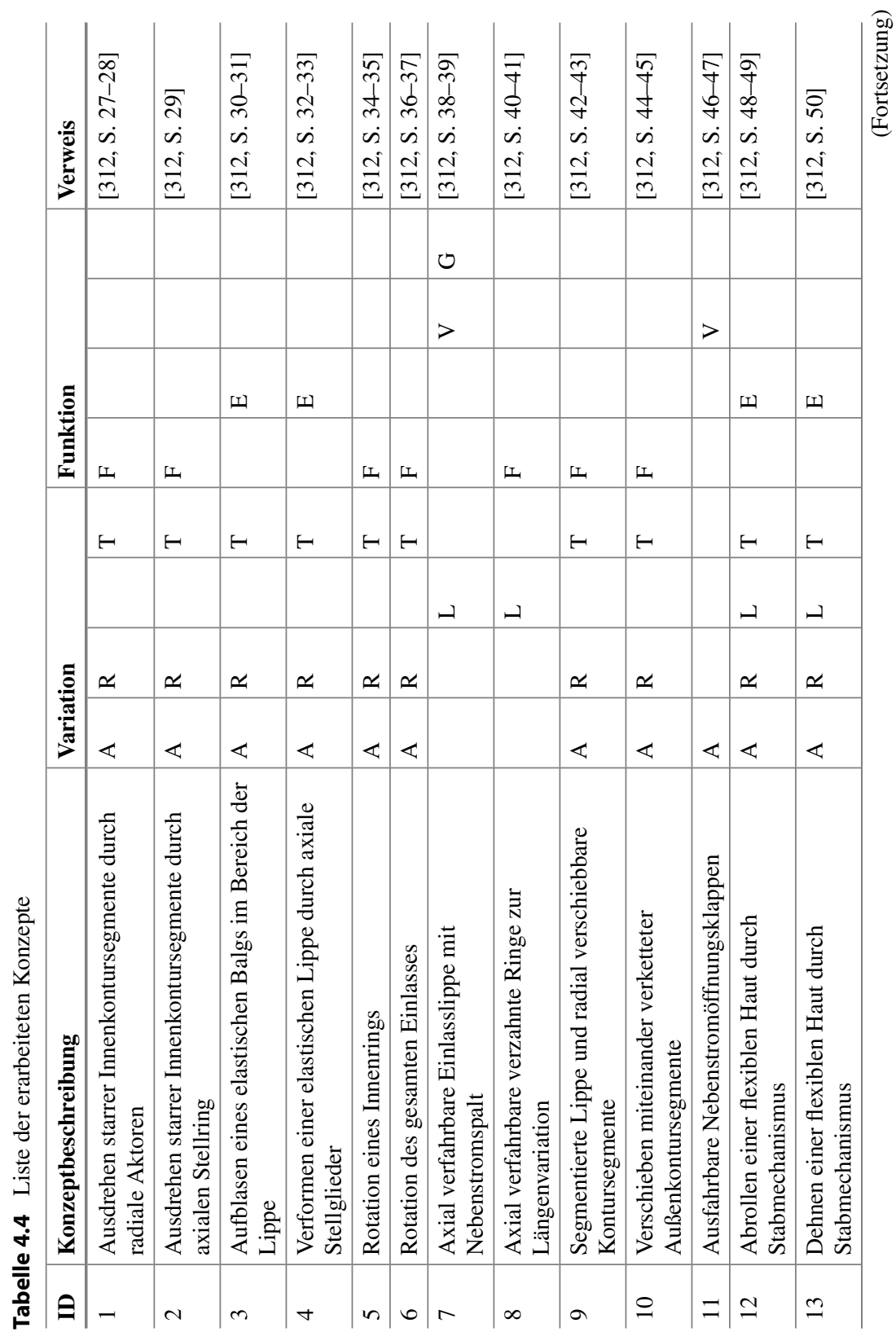




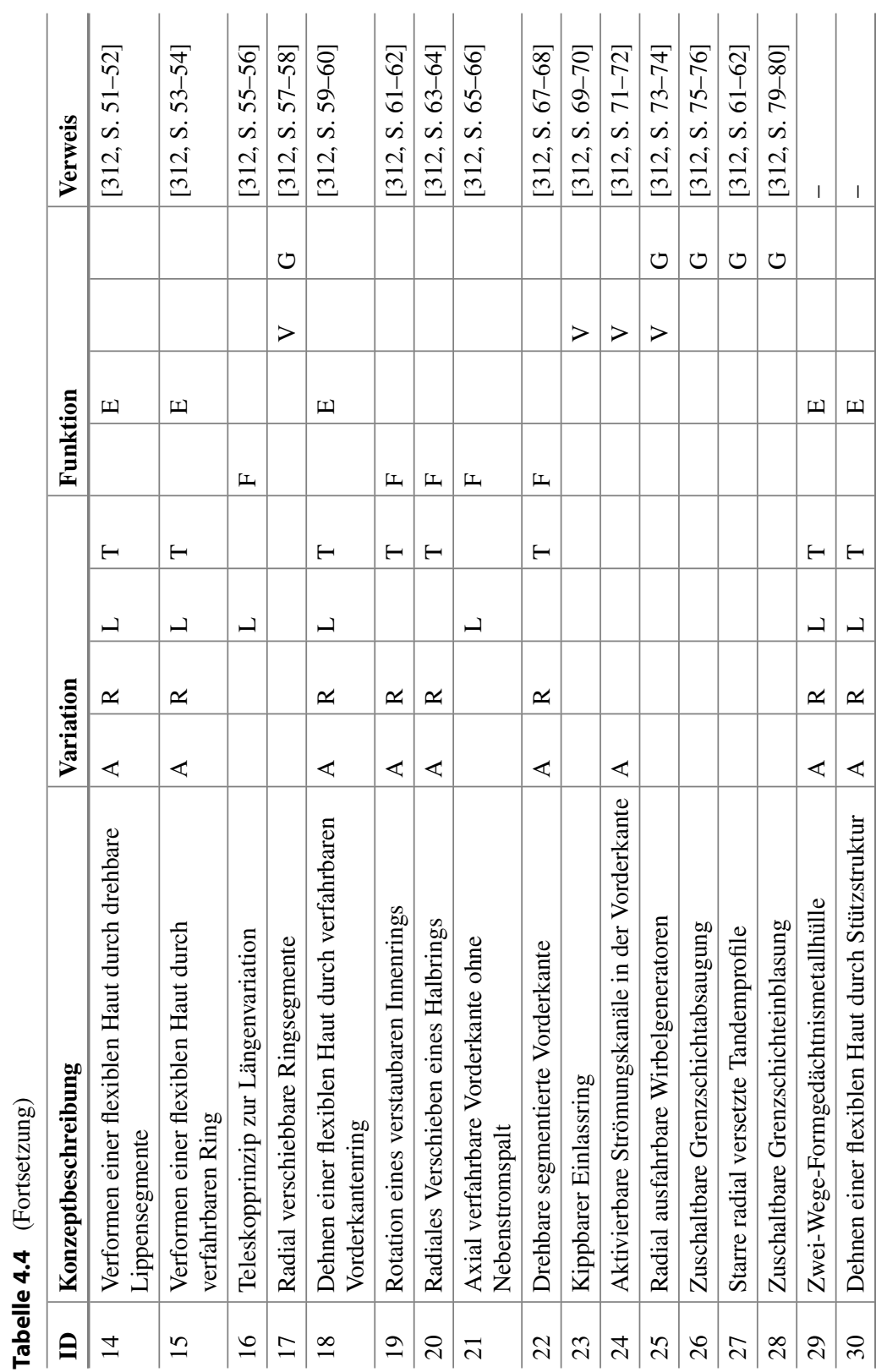


17 (Abbildung A.17) und in starrer Form in Konzept 27 (Abbildung A.27) vorgesehen. Öffnungsklappen, vgl. Abbildung 2.31 rechts, werden in Konzept 11 (Abbildung A.11) eingesetzt. In Konzept 25 (Abbildung A.25) wird das Prinzip der Wirbelgeneratoren aufgegriffen.

Die aktive Grenzschichtbeeinflussung, vgl. Abbildung 2.33, kommt in Form von Grenzschichtabsaugung in Konzept 26 (Abbildung A.26) und als Einblasen in die Grenzschicht in Konzept 28 (Abbildung A.28) zum Einsatz.

Die Konzepte 1 (Abbildung A.1), 2 (Abbildung A.2), 8-10 (Abbildung A.8Abbildung A.10) und 22 (Abbildung A.22) entsprechen grundlegend dem Prinzip der Verschiebung starrer Segmente, vgl. Abbildung 2.32. Die Konzepte 3 (Abbildung A.3), 4 (Abbildung A.4), 12-15 (Abbildung A.12-Abbildung A.15), 18 (Abbildung A.18), 29 (Abbildung A.29) sowie 30 (Abbildung A.30) verwenden vorwiegend elastisch verformbare Oberflächenmaterialien für die Konturvariation. Unterschiede zwischen den jeweiligen Konzepten sowie existierenden Patenten liegen beispielsweise in den Bereichen der möglichen Konturvariation, der Gestaltung der Vorderkante oder der Kinematik.

\subsubsection{Konzeptvorauswahl}

Die identifizierten Konzeptideen wurden entsprechend ihrer Erfüllung der qualitativen Ausschlusskriterien aus Abschnitt 4.1.4 bewertet, vgl. Tabelle 4.5. Bei der Bewertung der Konzepte wurde deren Erfüllung der Kriterien

- hohe Sicherheit (S),

- große Geometrievariation (V),

- geringe Beeinträchtigung der Umströmung (A),

- geringe Komplexität (K) und

- gute Integrierbarkeit (I)

unterschieden in

- bereits erfüllt (2),

- perspektivisch erfüllbar (1) und

- nicht erfüllbar (0). 
Tabelle 4.5 Bewertung der Konzepte zur Vorauswahl

\begin{tabular}{|c|c|c|c|c|c|c|c|}
\hline ID & Konzept & $\mathbf{S}$ & $\mathbf{V}$ & $\mathbf{A}$ & $\mathbf{K}$ & $\mathbf{I}$ & Ergebnis \\
\hline 1 & $\begin{array}{l}\text { Ausdrehen starrer Innenkontursegmente } \\
\text { durch radiale Aktoren }\end{array}$ & 1 & 1 & 1 & 1 & 1 & Geeignet \\
\hline 2 & $\begin{array}{l}\text { Ausdrehen starrer Innenkontursegmente } \\
\text { durch axialen Stellring }\end{array}$ & 1 & 1 & 1 & 1 & 1 & Geeignet \\
\hline 3 & $\begin{array}{l}\text { Aufblasen eines elastischen Balgs im } \\
\text { Bereich der Lippe }\end{array}$ & 0 & 1 & 1 & 1 & 1 & Ungeeignet \\
\hline 4 & $\begin{array}{l}\text { Verformen einer elastischen Lippe durch } \\
\text { axiale Stellglieder }\end{array}$ & 0 & 1 & 2 & 1 & 1 & Ungeeignet \\
\hline 5 & Rotation eines Innenrings & 1 & 0 & 1 & 0 & 1 & Ungeeignet \\
\hline 6 & Rotation des gesamten Einlasses & 1 & 0 & 1 & 0 & 1 & Ungeeignet \\
\hline 7 & $\begin{array}{l}\text { Axial verfahrbare Einlasslippe mit } \\
\text { Nebenstromspalt }\end{array}$ & 1 & 1 & 0 & 1 & 2 & Ungeeignet \\
\hline 8 & $\begin{array}{l}\text { Axial verfahrbare verzahnte Ringe zur } \\
\text { Längenvariation }\end{array}$ & 1 & 0 & 1 & 1 & 1 & Ungeeignet \\
\hline 9 & $\begin{array}{l}\text { Segmentierte Lippe und radial } \\
\text { verschiebbare Kontursegmente }\end{array}$ & 1 & 1 & 1 & 1 & 1 & Geeignet \\
\hline 10 & $\begin{array}{l}\text { Verschieben miteinander verketteter } \\
\text { Außenkontursegmente }\end{array}$ & 1 & 2 & 1 & 1 & 1 & Geeignet \\
\hline 11 & $\begin{array}{l}\text { Ausfahrbare } \\
\text { Nebenstromöffnungsklappen }\end{array}$ & 1 & 1 & 0 & 1 & 1 & Ungeeignet \\
\hline 12 & $\begin{array}{l}\text { Abrollen einer flexiblen Haut durch } \\
\text { Stabmechanismus }\end{array}$ & 0 & 1 & 1 & 0 & 1 & Ungeeignet \\
\hline 13 & $\begin{array}{l}\text { Dehnen einer flexiblen Haut durch } \\
\text { Stabmechanismus }\end{array}$ & 0 & 1 & 1 & 0 & 1 & Ungeeignet \\
\hline 14 & $\begin{array}{l}\text { Verformen einer flexiblen Haut durch } \\
\text { drehbare Lippensegmente }\end{array}$ & 1 & 1 & 1 & 1 & 1 & Geeignet \\
\hline 15 & $\begin{array}{l}\text { Verformen einer flexiblen Haut durch } \\
\text { verfahrbaren Ring }\end{array}$ & 0 & 1 & 1 & 1 & 1 & Ungeeignet \\
\hline 16 & Teleskopprinzip zur Längenvariation & 1 & 0 & 1 & 1 & 1 & Ungeeignet \\
\hline 17 & Radial verschiebbare Ringsegmente & 1 & 1 & 0 & 1 & 2 & Ungeeignet \\
\hline 18 & $\begin{array}{l}\text { Dehnen einer flexiblen Haut durch } \\
\text { verfahrbaren Vorderkantenring }\end{array}$ & 2 & 1 & 2 & 1 & 1 & Geeignet \\
\hline 19 & Rotation eines verstaubaren Innenrings & 1 & 1 & 1 & 0 & 1 & Ungeeignet \\
\hline 20 & Radiales Verschieben eines Halbrings & 1 & 0 & 1 & 1 & 1 & Ungeeignet \\
\hline
\end{tabular}


Tabelle 4.5 (Fortsetzung)

\begin{tabular}{l|l|l|l|l|l|l|l}
\hline ID & Konzept & S & V & A & K & I & Ergebnis \\
\hline 21 & $\begin{array}{l}\text { Axial verfahrbare Vorderkante ohne } \\
\text { Nebenstromspalt }\end{array}$ & 1 & 0 & 1 & 1 & 1 & Ungeeignet \\
\hline 22 & Drehbare segmentierte Vorderkante & 1 & 2 & 1 & 1 & 1 & Geeignet \\
\hline 23 & Kippbarer Einlassring & 1 & 0 & 1 & 1 & 1 & Ungeeignet \\
\hline 24 & $\begin{array}{l}\text { Aktivierbare Strömungskanäle in der } \\
\text { Vorderkante }\end{array}$ & 0 & 1 & 0 & 2 & 2 & Ungeeignet \\
\hline 25 & Radial ausfahrbare Wirbelgeneratoren & 1 & 1 & 0 & 2 & 2 & Ungeeignet \\
\hline 26 & Zuschaltbare Grenzschichtabsaugung & 1 & 1 & 1 & 1 & 1 & Geeignet \\
\hline 27 & Starre radial versetzte Tandemprofile & 1 & 0 & 0 & 1 & 2 & Ungeeignet \\
\hline 28 & Zuschaltbare Grenzschichteinblasung & 1 & 1 & 1 & 1 & 1 & Geeignet \\
\hline 29 & Zwei-Wege-Formgedächtnismetallhülle & 1 & 1 & 1 & 1 & 0 & Ungeeignet \\
\hline 30 & $\begin{array}{l}\text { Dehnen einer flexiblen Haut durch } \\
\text { Stützstruktur }\end{array}$ & 1 & 1 & 1 & 1 & 1 & Geeignet \\
\hline
\end{tabular}

Zellwerte: Kriterium wird bereits erfüllt (2), perspektivisch erfüllbar (1), nicht erfüllbar (0)

Der geringe Detailgrad der Konzepte aufgrund des frühen Entwicklungsstadiums wurde bei der Bewertung berücksichtigt. Aus diesem Grund gilt eine Konzeptvariante nur dann als ungeeignet, wenn sie mindestens eines dieser Kriterien perspektivisch nicht erfüllen kann.

Um eine möglichst objektive Vorauswahl zu ermöglichen, führten sechs Luftfahrtingenieure die Bewertung unabhängig voneinander durch. Bei voneinander abweichenden Bewertungsergebnissen wurde eine gemeinsame Lösung gefunden. Der verbleibende subjektive Einfluss bei der Konzeptbewertung wird durch die gewichtete Punktbewertung im späteren Verlauf vermindert.

Konzepte, deren Vorderkante aus einem elastischen, weichen Material besteht, würden bei möglichen Vogelschlägen oder Erosionseinflüssen mit großer Wahrscheinlichkeit versagen. Dies kann größere Sicherheitsrisiken nach sich ziehen, weshalb diese Konzepte aussortiert wurden. Dies betrifft die Konzepte 3, 4, 12, 13, 15 und 24. Hingegen wurden Konzepte, die in einer Ausweisung des Einlasses als Feuerzone resultieren würden, mit perspektivisch erfüllbar bewertet, da dieses Risiko durch konstruktive Maßnahmen beherrschbar ist. Hierzu ist beispielsweise Konzept $1 \mathrm{zu}$ zählen.

Ein geringes aerodynamisches Einsparpotenzial wäre die Folge einer zu geringen Geometrievariation durch das Konzept. Aus diesem Grund wurden Konzepte, 
die ausschließlich die Einlasslage (5, 6 und 23), die Einlasslänge (8, 16, 21), nur einen Einlasssektor (20) oder keine geometrischen Parameter variieren können (27), aussortiert.

Die Umströmung von Klappen und Profilen, die aus der Einlasskontur herausstehen, führt zu einer stark turbulenten Umströmung. Aufgrund der negativen Folgen für das Einlassdruckverhältnis und die Lärmbelastung infolge der Turbulenzen wurde von diesen Konzepten abgesehen. Hierzu gehören die Konzepte 7, $11,17,24,25$ und 27.

Die abschließende Komplexität eines Konzeptes ist zu diesem frühen Zeitpunkt sehr schwer zu beurteilen. Jedoch wurden hierbei Konzepte ausgeschlossen, die den gesamten Einlass oder einen Innenring rotieren (5, 6 und 19) oder die eine aufwendige Stützmechanik für die flexible Vorderkante erfordern (12 und 13).

Eine Integrierbarkeit in den Einlass wird allen Konzepten bis auf Konzept 29, das eine Zwei-Wege-Formgedächtnismetallhülle verwendet, zugetraut. Dieses Konzept weist einen hohen Bauraum- und Energiebedarf auf. Zudem erfordert es eine komplexe Temperaturregelung für das breite Spektrum möglicher auftretender Umgebungstemperaturen.

Darüber hinaus offenbaren die Ergebnisse der Konzeptvorauswahl, dass noch keine ideale Lösung gefunden wurde. Während der Bewertung wurden zahlreiche Schwachstellen der jeweiligen Konzepte identifiziert und zugleich Lösungsmöglichkeiten erarbeitet. Weiterhin wurden Konzepte mit ähnlichen Lösungsprinzipen zusammengefasst und teilweise miteinander kombiniert, um zusätzliche Konzepte zu generieren. Dadurch entstanden Konzeptgruppen, die im weiteren Verlauf der Konzeptstudie detailliert untersucht wurden, um das bestgeeignete Lösungsprinzip für die Geometrievariation von Triebwerkseinlässen zu identifizieren. Die jeweiligen Konzeptgruppen beschreiben Konzepte zur Variation der Einlassgeometrie durch

- Verschieben starrer Komponenten einer segmentierten Kontur und/oder Vorderkante $(1,2,9,10$ und 12),

- Verformen eines elastischen Oberflächenmaterials (14, 18 und 30), sowie

- aerodynamische Grenzschichtbeeinflussung $(26,28)$.

\subsubsection{Konzeptgruppen}

Nachfolgend wird die prinzipielle Funktionsweise der jeweiligen Konzeptgruppen anhand eines Beispielkonzepts vorgestellt. Zudem werden die grundlegenden 
Herausforderungen bei der Umsetzung sowie die Vorteile der jeweiligen Gruppen erläutert.

Detaillierte Herausforderungen der Konzeptgruppen werden mit den Ergebnissen der vorläufigen Systemsicherheitsanalyse und der Analyse von Fehlern gemeinsamer Ursachen in den anschließenden Kapiteln beschrieben. Basierend auf diesen Ergebnissen werden darauffolgend die Integrationsstudien zur Auswahl der jeweiligen Lösungen für Stellsystem, Vereisungsschutz und Lärmminderung präsentiert, vgl. Abschnitt 4.4.2. Aus diesem Grund werden für diese genannten Funktionen bei den nachfolgenden Konzeptgruppen keine oder nur exemplarische Lösungsmöglichkeiten angegeben.

\section{Verschieben starrer Komponenten}

Ein Konzept aus der Gruppe, bei der die Einlassgeometrie durch Neupositionierung starrer Segmente der Außenhülle angepasst wird, ist in Abbildung 4.12 veranschaulicht. Dieses Konzept basiert auf einer Kombination und Weiterentwicklung der Konzepte 2 (Abbildung A.2) und 10 (Abbildung A.10).

Schnittansicht der Seitenansicht

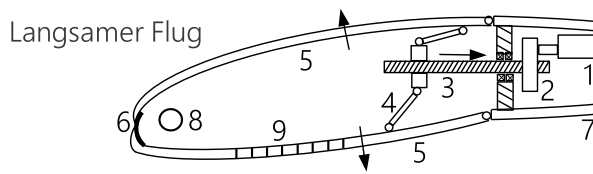

Schneller Reiseflug

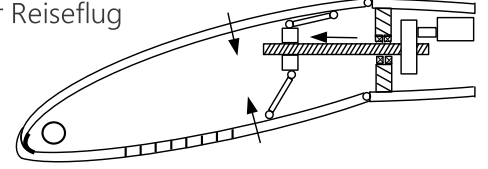

Legende:

1 - Elektromotor

2 - Getriebe

3 - Gewindetriebspindel und -mutter

4 - Stellstab

5 - Einlasssegmente

6 - Flexible Abdichtung

7 - Statische Gondel

8 - Zapfluftleitung

9 - Akustische Auskleidung

Abbildung 4.12 Geometrievariation durch Verschieben starrer Segmente

Das vorgestellte Konzept variiert die Innen- und Außenkontur des Einlasses durch axiale Bewegung eines Stellglieds. Dieses Konzept findet zudem Verwendung im ersten von zwei Funktionsdemonstratoren, vgl. Abschnitt 4.5.4. Als Stellglied kann beispielsweise die Mutter eines Gewindetriebs (3) genutzt werden, welcher durch einen Elektromotor (1) und ein zwischengeschaltetes Getriebe (2) angetrieben wird. Durch die axiale Bewegung der Gewindetriebmutter (3) wird die Lage gelenkig gekoppelter Stellstäbe (4) gesteuert. Die Stellstäbe (4) sind wiederum gelenkig mit den konturgebenden Einlasssegmenten (5) verbunden, welche durch die Bewegung ebenfalls ihre Lage verändern, wodurch die Außenkontur des 
Einlasses angepasst wird. Im Bereich der Vorderkante verhindert eine elastische Abdichtung (6) die Entstehung von Spalten zwischen den Kontursegmenten (5) der Innen- und Außenseite des Einlasses.

Für den Vereisungsschutz und die Lärmreduktion können alle Lösungsmöglichkeiten in Betracht gezogen werden. Jedoch sollten Synergien zwischen Teilsystemen maximiert und Kombinationen vermieden werden, die zu einem erhöhten Feuerrisiko beitragen können.

Mit dem beschriebenen Konzept können relevante Strömungsquerschnitte und die gewünschte Einlassdicke eingestellt werden. Ermöglicht wird diese Variation durch die Segmentierung des Einlasses über den Umfang, vgl. Abbildung 4.8 in Abschnitt 4.2.4. Die Segmentierung der Kontur über den Umfang ist das Hauptmerkmal der gesamten vorliegenden Konzeptgruppe. Die Entstehung von Umfangsspalten wird mittels geradliniger Segmentverlängerungen umgangen, vgl. Abbildung 4.11. Dabei sollte die Ansammlung von Schmutz und Eis zwischen den Segmenten vermieden werden, um die Funktionsfähigkeit des variablen Einlasses nicht zu gefährden.

Aufgrund des festen Radius der Segmente kann nur für einen Flugzustand eine exakte Kreisquerschnittsfläche eingestellt werden. Durch eine Erhöhung der Anzahl der Umfangssegmente kann eine nahezu kreisrunde Geometrie erreicht werden. Dies resultiert in einer gleichförmigeren Fananströmung, erhöht jedoch auch die Komplexität des Systems.

Ein größerer Variationsbereich der genannten Geometrieparameter als im vorgestellten Konzept kann durch eine zusätzliche axiale Segmentierung der Außenkontur realisiert werden. Diese ist beispielsweise bei den Konzepten 9 (Abbildung A.9) und 10 (Abbildung A.10) vorgesehen. Damit einhergehend sind jedoch auch eine erhöhte Bauteilanzahl, eine höhere Komplexität sowie mehr Spalte zwischen den Segmenten, wodurch die Zuverlässigkeit negativ beeinflusst werden kann.

Andere Konzepte dieser Konzeptgruppe können zusätzlich die Länge des Einlasses anpassen, beispielsweise durch Nutzung des Teleskopprinzips. Die Anpassung der Einlasslänge ermöglicht zwar bessere aerodynamische Eigenschaften, erfordert jedoch zugleich eine erhöhte Masse und Komplexität der Konstruktion.

Durch die Variation der Kontur verändert sich die Lage von Komponenten und der vorhandene Bauraum innerhalb des Einlasses. Üblicherweise unbewegliche Komponenten, wie Kabel und Rohrleitungen, können dadurch zusätzlichen Beanspruchungen ausgesetzt sein. Um Beschädigungen dieser Komponenten zu vermeiden, werden sie flexibel gestaltet und ein Mindestabstand zwischen den Komponenten zur Vermeidung von Kollisionen vorgesehen. 


\section{Verformen eines elastischen Oberflächenmaterials}

Ein Vertreter der Konzeptgruppe, die die Einlassgeometrie durch Verformung eines elastischen Oberflächenmaterials anpasst, ist in Abbildung 4.13 dargestellt. Hierbei handelt es sich um eine Weiterentwicklung von Konzept 14, vgl. Abbildung A.14, bei der sowohl die Einlassdicke, relevante Strömungsquerschnitte als auch die Länge angepasst werden können. Bei diesem Konzept wird die Antriebsenergie eines Elektromotors (1) über ein Getriebe (2) und einen Gewindetrieb (3) in eine Axialbewegung der Gewindetriebmutter umgewandelt. Durch die axiale Bewegung der Gewindetriebmutter wird das elastische Oberflächenmaterial des Einlasses (5) durch Stellstäbe (4) verformt. Weiterhin dreht sich die Vorderkante (6) durch die Axialbewegung der Stellstäbe (4) in eine Position, die für die vorherrschende Flugphase geeignet ist. Gleichzeitig dienen die Stellstäbe (4) als Stützstruktur, die die Geometrie der elastischen Oberfläche (5) und die Lage der Vorderkante (6) sicherstellt.

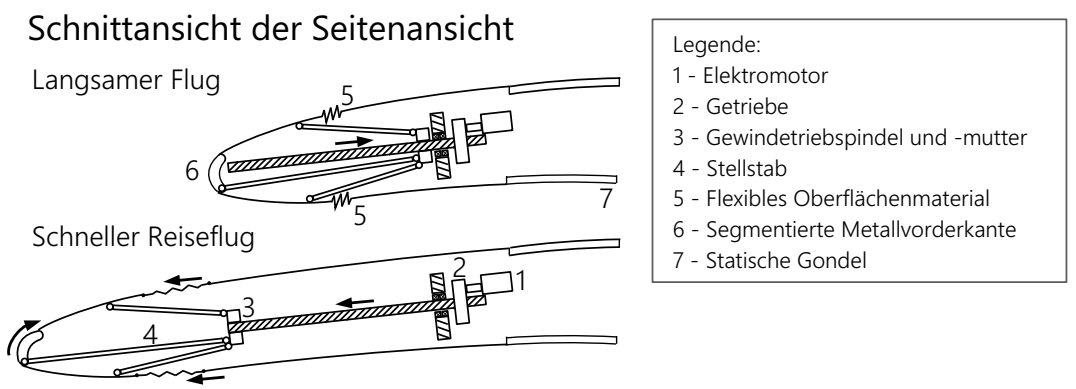

Abbildung 4.13 Geometrievariation durch elastische Oberflächenverformung

Die Vorderkante dieses Konzepts ist aus Aluminium gefertigt, um Beanspruchungen wie Erosion und Vogelschlägen standzuhalten. Zusätzlich ist die Vorderkante über den Umfang segmentiert, um die Anpassung des Eintrittsquerschnitts und der Lippendicke zu ermöglichen. Zwischen diesen Segmenten sind wiederum komplexe Abdichtelemente erforderlich [314, S. 87-90], vgl. Abschnitt 4.2.4. Bei Verwendung einer starren Lippe ist eine größere Anpassung des Eintrittsquerschnitts nicht möglich, jedoch kann dadurch die strukturelle Stabilität deutlich gesteigert und die Komplexität signifikant gesenkt werden, vgl. Konzept 18 (Abbildung A.18).

Als elastisches Oberflächenmaterial können Elastomere, elastisch verformbare Verbundwerkstoffe oder intelligente Werkstoffe verwendet werden, vgl. 
Abschnitt 2.4.3. Für die Verformung des Materials sind stets Stell- und Stützmechanismen oder bei Verwendung intelligenter Werkstoffe die Erzeugung externer Reize erforderlich.

Eine Segmentierung der elastischen Oberfläche über den Umfang der Kontur ist nicht vorgesehen. Daher muss das elastische Material auch in Umfangsrichtung belastbar und anpassbar sein. Daraus ergeben sich anspruchsvollere mechanische Anforderungen an das flexible Material, die im MorphElle-Projekt beispielsweise durch eine elastische Verbundmaterialhaut erfüllt werden sollten [23], [24], [26].

Darüber hinaus kann die Lebensdauer der elastischen Materialien stark eingeschränkt sein. Dies begründet sich aus der zyklischen Verformung in Kombination mit den auftretenden Beanspruchungen, z. B. Erosion, und den Umgebungsbedingungen in der Luftfahrt, z. B. Temperaturen. Folglich können Beschichtungen für Erosionsbeständigkeit, Blitzschutz, Isolierung und Vereisungsschutz erforderlich werden. Weiterhin ist eine Stützstruktur zur Aufnahme von Vogelschlagbelastungen erforderlich.

Ein möglicher Lebensdauereinfluss durch einen Temperatureintrag bei Verwendung von Heißlufteisschutzsystemen oder elektrothermischen Eisschutzsystemen ist zu untersuchen. Dadurch müssen ggf. Eisschutzsysteme mit geringerem Erprobungsgrad und höherer Ausfallwahrscheinlichkeit eingesetzt werden. Andererseits könnte der Stellmechanismus auch als redundantes elektromechanisches Eisschutzsystem im Fall einer Fehlfunktion des Primärsystems eingesetzt werden, indem er die Kontur minimal variiert.

Da konventionelle starre akustische Auskleidungen eine Konturanpassung verhindern, sollten entweder akustische Auskleidungen mit flexiblen Wänden oder die Schallauslöschung durch Gegenschall als Lärmminderungsmaßnahmen in Betracht gezogen werden. Auch die Gestaltung von Kabeln und Leitungen ist flexibel durchzuführen und Kollisionen zwischen Komponenten sind zu vermeiden.

Der Hauptvorteil dieser Gruppe ist die spalt- und stufenfreie Oberfläche, da diese aus einem Stück gefertigt werden kann. Die Limitierungen dieser Konzeptgruppe liegen in der Notwendigkeit einer tragenden Struktur zur Sicherstellung, dass die Geometrie im gewünschten Zustand verbleibt, sowie in der reduzierten Lebensdauer des elastischen Oberflächenmaterials. Weiterhin bedarf der Schutz vor Vereisung und die Minderung des Fanlärms erhöhter Aufmerksamkeit.

\section{Aerodynamische Grenzschichtbeeinflussung}

Von den in Abschnitt 2.4.2 vorgestellten Möglichkeiten zur Vermeidung von Strömungsablösungen durch Grenzschichtbeeinflussung verbleiben nach der Konzeptvorauswahl lediglich zwei aktive Varianten durch 
- Absaugen der Grenzschicht und

- tangentiales Einblasen in die Grenzschicht, vgl. Abbildung 2.33.

Die erste Variante wird in Konzept 26 eingesetzt und scheidet bzw. saugt die verzögerte Strömungsgrenzschicht durch Absaugschlitze in der Einlasshülle ab, vgl. Abbildung A.26. Die abgesaugte Luft der Strömungsgrenzschicht kann gleichzeitig zur Ventilation der Triebwerksgondel verwendet werden. Das Prinzip der Grenzschichtabsaugung findet in der Luftfahrt bereits vielfach Anwendung [328], [329].

Bei der zweiten Variante, dem Grenzschichteinblasen, wird die Geschwindigkeitsdifferenz zwischen Wand und Strömung durch tangentiales Einblasen in die Grenzschicht minimiert, vgl. Konzept 28 (Abbildung A.28). Die größte Herausforderung bei diesem Konzept besteht darin, bei jeder Flugbedingung die geeignete einzublasende Luftmenge mit geeigneter Geschwindigkeit, Druck und Temperatur zu bestimmen und dabei eine hohe Gleichförmigkeit der Fananströmung einzustellen. Geringfügige Fehler hierbei können zu Strömungsablösungen oder zur Beschädigung des Verdichters aufgrund erhöhter Temperaturen führen, was gefährliche Ereignisse zur Folge haben kann. Daher ist auch der Schutz der Einblasschlitze vor Verblockung durch Vereisung oder andere Einflüsse von großer Bedeutung.

Abgesehen von den verschließbaren Absaug- bzw. Einblasschlitzen weisen die Konzepte eine stufen- und lückenlose Oberfläche auf, was sich positiv auf die aerodynamischen Kennwerte des Einlasses auswirkt. Beide Varianten dieser Konzeptgruppe verwenden eine starre Einlassgeometrie mit minimalen Abmessungen und somit minimaler Masse. Hierfür wird die aerodynamisch ideale Geometrie für den schnellen Reiseflug gewählt, vgl. Abschnitt 2.2.1. Bei langsamem Flugzuständen mit den zugehörigen Fangstromröhren werden Strömungsablösungen durch die zuschaltbare Grenzschichtbeeinflussung vermieden. Diese wird über die axial und über den Umfang im Einlass verteilten Absaug- bzw. Einblasschlitze ermöglicht. Die ideale Verteilung dieser Schlitze ist in späteren Entwicklungsphasen zu ermitteln.

Die Konzepte erlauben den Einsatz konventioneller akustischer Auskleidungen und Eisschutzsysteme. Zudem wird die Verwendung eines großen und schweren Stellmechanismus einschließlich Aktorik vermieden. Allerdings ist für das Öffnen und Schließen der Absaug- bzw. Einblasschlitze eine Regelung erforderlich, beispielsweise über elektrisch ansteuerbare Ventile. Das Öffnen und Schließen der Schlitze muss mit hoherZuverlässigkeit erfolgen, da eine Fehlfunktion der jeweiligen Schlitze zu einer ungleichförmigen Fananströmung führen und letztlich gefährliche Ereignisse auslösen kann. Daher sind die Schlitze vor Schmutz, Feuchtigkeit, Fremdkörperschäden sowie Vereisung zu schützen. Darüber hinaus müssen versagenssichere Konstruktionsprinzipien, z. B. Redundanz, verwendet werden, um Strömungsablösungen im Fehlerfall zu vermeiden. Zudem muss die wesentlich dünnere Einlassgeometrie weiterhin Vogelschlägen standhalten können. 


\subsubsection{Fehlerbaumanalyse}

Basierend auf den Ergebnissen der Gefährdungsanalyse waren Fehler zu analysieren, die zur Einstellung einer unerwünschten Geometrie bei mehreren Triebwerken führen könnten, vgl. Abschnitt 4.2.3. Hierfür wäre die mögliche Folge mit den größtmöglichen Auswirkungen der Schubverlust des Flugzeugs. Die Fehlerbaumanalyse beinhaltet die Ermittlung und logische Verknüpfung aller Ausfälle, die zu diesem Ereignis führen können [273, S. 167].

$\mathrm{Zu}$ einem sicherheitskritischen Schubverlust des Flugzeugs führen nur Ereignisse, bei denen mindestens zwei der Triebwerke deutlich vom geforderten Wert abweichenden Schub erzeugen. Der Leistungsausfall eines einzelnen Triebwerks hingegen wäre durch das verbleibende Triebwerk bzw. je nach Flugzeugbauart die verbleibenden Triebwerke zu kompensieren.

Die Ursachen für den Schubverlust eines Triebwerks können vielfältiger Natur sein. Beispiele hierfür sind

- ausbleibende Brennstoffzufuhr,

- ein Rotorwellenbruch,

- ein Fanschaufelverlust,

- ein unkontrolliertes Feuer oder

- Verdichterpumpen.

Wie in den vorherigen Kapiteln beschrieben, kann Verdichterpumpen durch eine unerwünschte Einlassgeometrie, die nicht für die vorherrschende Flugphase geeignet ist, hervorgerufen werden. Eine unerwünschte Einlassgeometrie kann Folge verschiedener Fehler sein. Möglichkeiten hierfür sind

- Fehler des Stellmechanismus,

- Fehler des Kontrollsystems,

- Eisansammlungen und

- strukturelle Beschädigungen.

Die logische Verknüpfung dieser Fehlerfälle mit dem Ereignis des Schubverlusts ist für Konzepte, die ein Aktorsystem mit Feststellmechanismus verwenden, in Abbildung 4.14 dargestellt. 


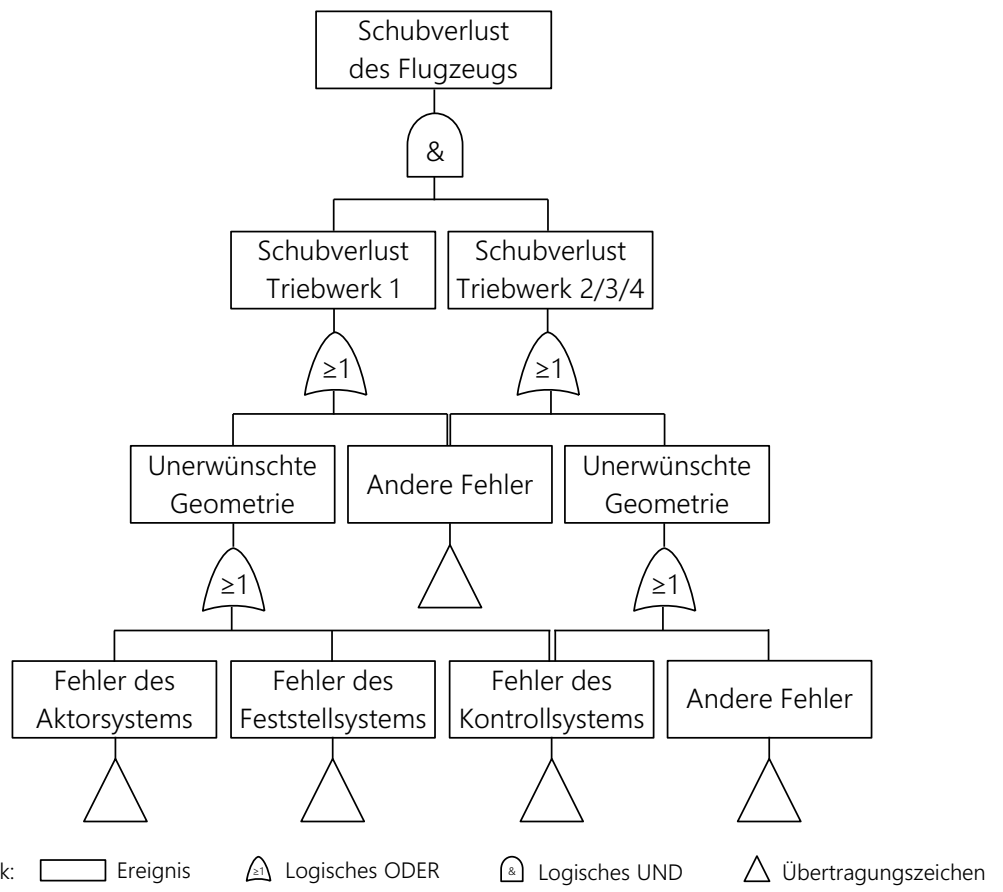

Symbolik: $\square$ Ereignis $\_$Logisches ODER

Abbildung 4.14 Fehlerbaum für den Schubverlust mehrerer Triebwerke

Fehler des Stellmechanismus können je nach Konzept beispielsweise ein Versagen der Aktorik, etwaiger Stellstäbe oder eventuell genutzter Ventile sein. Diese Fehler sollten unabhängig voneinander auftreten und somit nicht zeitgleich in mehreren Triebwerken. Dies wird im Rahmen der Analyse von Fehlern gemeinsamer Ursachen in Abschnitt 4.4.1 gezeigt.

Auch Eisansammlungen und strukturelle Beschädigungen, beispielsweise durch einzelne Vogelschläge hervorgerufen, sollten nicht zeitgleich mehrere Triebwerke betreffen. Dies wird einerseits durch das verwendete Eisschutzsystem und andererseits durch die konstruktive Gestaltung gewährleistet. Simultane Einschläge von Vögeln mit mehr als $2 \mathrm{~kg}$ Masse auf mehrere Triebwerke können und müssen nicht abgesichert werden [34, 25.631].

Ein Fehler des Kontrollsystems, beispielsweise durch ein fehlerhaftes Stellsignal, könnte ebenfalls mehrere Triebwerke gleichzeitig betreffen, vgl. Abbildung 2.35. Aufgrund der geforderten niedrigen Eintrittswahrscheinlichkeit eines 
Schubverlusts, ist die Integration einer zusätzlichen Sicherheitsmaßnahme zielführend, um die Auswirkungen von Fehlern des Kontrollsystems zu vermindern.

Aus diesem Grund sollten die Konzepte, die ein Aktorsystem verwenden, einen unabhängig ansteuerbaren Feststellmechanismus in Form eines Verschlusssystems beinhalten. Die Ansteuerung des Verschlusssystems hat hierbei unabhängig von der Ansteuerung des Aktors durch die zugehörige Steuerung zu erfolgen. Das Verschlusssystem könnte beispielsweise zwischen Motor und Getriebe integriert sein und somit die Bewegung des Aktors durch ein fehlerhaftes Stellsignal verhindern, vgl. Abbildung 4.15. Auf diesem Weg wird das Einstellen einer unerwünschten Geometrie und die Möglichkeit des Schubverlusts vermieden.

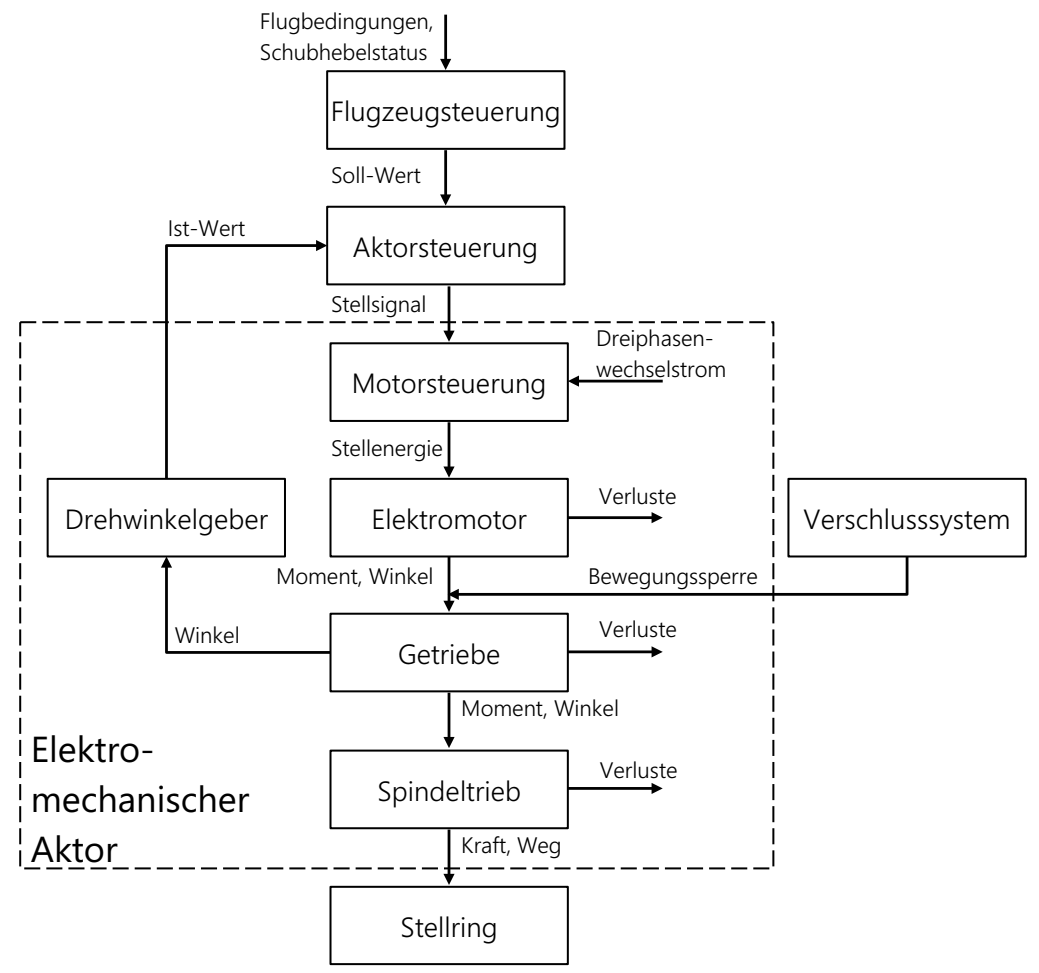

Abbildung 4.15 Elektromechanischer Aktor mit Feststellmechanismus 
Weitere Lösungsansätze für die redundante Sicherstellung der Ansteuerung von Aktoren in Luftfahrtsystemen werden von Rossow et al. [77, S. 748-749] und Grasselt [54, S. 94] vorgestellt. Hierzu zählt beispielsweise die Verwendung unterschiedlicher Energiequellen und Transportwege.

Für die Konzeptgruppe der aerodynamischen Grenzschichtbeeinflussung ist der gewählte Ansatz eines unabhängigen Feststellmechanismus aufgrund der eingesetzten elektrisch angesteuerten Ventile nicht zielführend. Eine Lösungsmöglichkeit für diese Konzeptgruppe besteht darin, im Rahmen von Tests nachzuweisen, dass auch die aerodynamisch ungünstigste Geometrie des Systems, die gleichzeitig der Geometrie beim Ausfall der Grenzschichtbeeinflussung entspricht, bei allen möglichen Flugzuständen funktionsfähig ist [34, 25.941]. Dies impliziert, dass die Funktionalität der Überschallgeometrie auch bei Startbedingungen nachzuweisen ist. Durch diesen Ansatz würde das aerodynamische Einsparpotenzial dieser Konzeptgruppe voraussichtlich signifikant verringert werden. Ein alternativer Ansatz hierzu wäre die doppelte Ausführung und unabhängige Ansteuerung aller vorhandenen Absaug- bzw. Einblasschlitze.

\subsection{Vorentwurf der Konzeptgruppen}

Um die Konzeptgruppe, die am besten für eine Umsetzung variabler Einlässe geeignet ist, zu identifizieren, waren weitere Analysen erforderlich, deren Ergebnisse nachfolgend vorgestellt werden. Dies erfolgt zuerst für die Analyse von Fehlern gemeinsamer Ursachen (CCA), bestehend aus

- der Analyse besonderer Risiken (PRA),

- der Zonensicherheitsanalyse (ZSA) sowie

- der Analyse redundanzüberbrückender Fehler (CMA).

Danach wird die Identifikation geeigneter Teilsysteme, insbesondere für den Schutz vor Vereisung, erläutert. Anschließend wird der mögliche Funktionsumfang der jeweiligen Konzeptgruppen, basierend auf den idealen und den umsetzbaren Einlassgeometrien, präsentiert. Die gesammelten Ergebnisse der durchgeführten Analysen ermöglichten eine abschließende Bewertung der jeweiligen Konzeptgruppen, wodurch eine Konzeptgruppe für weiterführende Untersuchungen ausgewählt werden konnte. 


\subsubsection{Analyse von Fehlern gemeinsamer Ursachen}

\section{Analyse besonderer Risiken}

Zahlreiche der in Abschnitt 3.2.4 aufgelisteten besonderen Risiken, wie beispielsweise Hagelschlag, haben einen vergleichbaren Einfluss auf variable wie auf starre Einlässe. Dennoch können die Auswirkungen vereinzelter Risiken durch die zusätzlichen Komponenten und die veränderte Bauweise variabler Einlässe deutlich größere Ausmaße annehmen. Hierbei sind insbesondere der Einfluss auf den Variationsmechanismus und die mögliche Segmentierung des Einlasses von Relevanz. Beispielsweise sind die Kontrollsysteme aller Konzeptgruppen vor elektromagnetischen Interferenzen zu schützen, um gefährlichen Ereignissen vorzubeugen.

Für die Konzepte, welche die Einlassgeometrie durch Bewegung starrer Segmente anpassen, gehören zusätzlich Eis, Reibung zwischen beweglichen Bauteilen, Vogelschlag und Blitzschlag zu den bedeutenden Risiken. Mögliche daraus resultierende Ereignisse, die Schwere der jeweiligen Folgen und die daraus abgeleiteten Anforderungen an die Eintrittswahrscheinlichkeit können Tabelle 4.6 entnommen werden.

Tabelle 4.6 Ausgewählte Ergebnisse der Analyse besonderer Risiken

\begin{tabular}{l|l|l|l|l}
\hline Risiko & Ereignisse & Mögliche Folgen & $\begin{array}{l}\text { Klassifizie- } \\
\text { rung }\end{array}$ & $\begin{array}{l}\text { Erlaubte } \\
\text { Ereignisse } \\
\text { pro } \\
\text { Flugstunde }\end{array}$ \\
\hline Eis & $\begin{array}{l}\text { Große } \\
\text { Ansammlungen } \\
\text { auf Einlässen } \\
\text { mehrerer } \\
\text { Triebwerke }\end{array}$ & $\begin{array}{l}\text { Abplatzendes Eis; } \\
\text { Beschädigungen des Fans; } \\
\text { Blockieren des } \\
\text { Stellmechanismus; } \\
\text { Strömungsbeeinträchtigungen; } \\
\text { Schubverlust mehrerer } \\
\text { Triebwerke }\end{array}$ & $\begin{array}{l}\text { Gefährlich } \\
\text { (Hazardous) }\end{array}$ & $<1 \mathrm{E-07}$ \\
\hline $\begin{array}{l}\text { Vogel- } \\
\text { schlag }\end{array}$ & $\begin{array}{l}\text { Einlass eines } \\
\text { Triebwerks }\end{array}$ & $\begin{array}{l}\text { Beschädigung von Segmenten; } \\
\text { Strömungsbeeinträchtigungen; } \\
\text { Beschädigungen des Fans; } \\
\text { Schubverlust eines Triebwerks; } \\
\text { Verlust von Segmenten; } \\
\text { Beschädigungen des Leitwerks; } \\
\text { Einschränkungen der Kontrolle } \\
\text { über das Flugzeug }\end{array}$ & $\begin{array}{l}\text { Gefährlich } \\
\text { (Hazardous) }\end{array}$ & $<1$ E-07 \\
\hline
\end{tabular}


Tabelle 4.6 (Fortsetzung)

\begin{tabular}{|c|c|c|c|c|}
\hline Risiko & Ereignisse & Mögliche Folgen & $\begin{array}{l}\text { Klassifizie- } \\
\text { rung }\end{array}$ & $\begin{array}{l}\text { Erlaubte } \\
\text { Ereignisse } \\
\text { pro } \\
\text { Flugstunde }\end{array}$ \\
\hline & $\begin{array}{l}\text { Einlässe mehrerer } \\
\text { Triebwerke }\end{array}$ & $\begin{array}{l}\text { Zusätzlich Schubverlust } \\
\text { mehrerer Triebwerke }\end{array}$ & $\begin{array}{l}\text { Gefährlich } \\
\text { (Hazardous) }\end{array}$ & $<1 \mathrm{E}-07$ \\
\hline $\begin{array}{l}\text { Blitz- } \\
\text { schlag }\end{array}$ & $\begin{array}{l}\text { Direkter Einschlag } \\
\text { auf ein Triebwerk }\end{array}$ & $\begin{array}{l}\text { Beschädigung von Segmenten } \\
\text { durch erhöhte Temperaturen; } \\
\text { Beschädigungen elektrischer } \\
\text { Komponenten, z. B. Aktoren } \\
\text { und Kontrollsysteme, durch } \\
\text { Überladung; } \\
\text { Verlust der Kontrolle über den } \\
\text { Variationsmechanismus; } \\
\text { Schubverlust eines Triebwerks }\end{array}$ & $\begin{array}{l}\text { Unbedeutend } \\
\text { (Minor) }\end{array}$ & $<1 \mathrm{E}-03$ \\
\hline $\begin{array}{l}\text { Rei- } \\
\text { bung }\end{array}$ & $\begin{array}{l}\text { Starke Reibung } \\
\text { zwischen } \\
\text { beweglichen Ein- } \\
\text { lasskomponenten } \\
\text { eines Triebwerks }\end{array}$ & $\begin{array}{l}\text { Lebensdauerverringerung; } \\
\text { Blockieren des } \\
\text { Stellmechanismus; } \\
\text { Schubverlust eines Triebwerks }\end{array}$ & $\begin{array}{l}\text { Unbedeutend } \\
\text { (Minor) }\end{array}$ & $<1 \mathrm{E}-03$ \\
\hline
\end{tabular}

In Abhängigkeit der gewählten Umsetzungen für den Vereisungsschutz und die Aktorik können zusätzliche Risiken, wie austretende Hydraulikflüssigkeit und Feuergefahr, relevant werden. Die größte Wahrscheinlichkeit dafür besteht beim Kombinieren eines Heißlufteisschutzssystems mit einem elektrohydraulischen Aktorsystem.

Während schwere Vereisungsbedingungen, wie gefrierender Regen, mit einer Wahrscheinlichkeit von $10^{-2}$ pro Flug auftreten, können normale Vereisungsbedingungen bei jedem Flug eintreffen [34, AMC 25.1419], [53, S. 164]. Daher sind bei modernen Flugzeugen Eisschutzsysteme stets Bestandteil des Einlasses. Diese Systeme decken zumeist nur den Bereich der Vorderkante und der Einlasslippe ab.

Bei segmentierten Einlasskonzepten können größere Eisansammlungen auch im Bereich des Diffusorkanals und der äußeren Beplankung eine Gefahr darstellen, indem sie sich zwischen den Segmenten bilden und somit den Stellmechanismus blockieren. Um die Variationsfähigkeit von Einlässen dieser Konzeptgruppe sicherzustellen, ist folglich eine Erweiterung der vom Eisschutzsystem abgedeckten Fläche erforderlich. Dies führt zu einer Erhöhung des erforderlichen Energiebedarfs, des technischen Aufwands sowie der anfallenden Betriebskosten. 
Die Wahl des Eisschutzsystems beeinflusst neben der Feuergefahr auch die Relevanz weiterer Risiken. Bei der Wahl elektrischer Eisschutzsysteme müssen die Einwirkungen von Blitzschlägen und elektromagnetischen Feldern genauer untersucht werden. Beim Einsatz von Heißlufteisschutzsystemen sind die Folgen von Schottwandbrüchen und die Leckage von Zapfluftkanälen detailliert zu untersuchen.

Vogelschläge sind ein bekanntes Risiko in der Luftfahrt. Etwa $37 \%$ der gemeldeten Vogelschläge betreffen dabei das Triebwerk oder dessen Einlass [309, S. 28]. Gemäß CS-25.631 [34] sind Vogelschläge als Extremlasten zu behandeln, was plastische Materialverformungen, nicht jedoch das strukturelle Versagen sicherheitskritischer Komponenten, erlaubt. Es ist sicherzustellen, dass ein einzelner Vogelschlag auf den Einlass nicht zu gefährlichen Folgen durch starke Leitwerksbeschädigungen und einen möglichen Kontrollverlust über das Flugzeug führt, vgl. Tabelle 4.6. Im Rahmen der strukturellen Dimensionierung in Abschnitt 4.5.2 und Anhang A.6 wird diese Sicherheit nachgewiesen. Dies erlaubt die Klassifizierung eines Vogelschlags auf ein einzelnes Triebwerk als unbedeutend (Minor). Jedoch erfordert die konstruktive Gestaltung gegen Vogelschlag eine Erhöhung des erforderlichen Bauraums und der Masse eines Konzepts.

In der Europäischen Union wird ein Flugzeug rein statistisch alle 2400 Flugstunden von einem Blitz getroffen [53, S. 165]. Negative Auswirkungen dieser Blitzeinschläge sind durch die Verwendung von temperaturbeständigen Materialien und die Gestaltung des Einlasses als Faraday'schen Käfig zu vermeiden. Außerdem müssen elektrische Komponenten geerdet werden.

Die Reibung zwischen den Einlasssegmenten oder anderen beweglichen Komponenten kann beispielsweise durch Gleitbeschichtungen oder eine lebenslange Schmierung reduziert werden.

Für die Konzeptgruppe der elastischen Oberflächenverformung sind die auftretenden Risiken und deren Folgen größtenteils vergleichbar. Lediglich das Risiko des Vogelschlags stellt eine größere Unsicherheit dar, da eine höhere Festigkeit der Konstruktion die Variationsfähigkeit des elastischen Oberflächenmaterials negativ beeinflussen könnte.

Bei der Konzeptgruppe der aerodynamischen Grenzschichtbeeinflussung ist insbesondere der Schutz der Absaug- bzw. Einblasschlitze vor Verschmutzungen und Eis zu beachten. Andernfalls könnten die Schlitze verstopfen, woraus gefährliche Folgen aufgrund von Strömungsablösungen resultieren würden.

\section{Zonensicherheitsanalyse}

Der erste Schritt der ZSA bestand in der Erstellung von Konstruktions- und Installationsrichtlinien für Komponenten, wie Rohre, Leitungen, Schläuche, Drähte und 
Kabel [57]. Beispielhafte Konstruktions- und Installationsrichtlinien, die aus der Zonensicherheitsanalyse hervorgehen, umfassen Anforderungen zur Minimierung von Spannungen, Blockaden und Flüssigkeitsansammlungen sowohl für statische als auch für bewegliche Teile. So ist eine Drainage in Bereichen zu installieren, in denen Flüssigkeitsansammlungen Brände, Korrosion oder Fäulnis verursachen können. Darüber hinaus sollten redundante Systeme durch einen Mindestabstand voneinander getrennt werden, um Ereignisse und Ausfälle zu vermeiden, die beide Systeme betreffen. Gleiches gilt für Zapfluftleitungen, Stellenergieleitungen oder Sensorkabel. Die Ansammlung von Staub und Schmutz auf den Komponentenoberflächen im Inneren der Einlassstruktur ist durch eine Ventilation zu verhindern.

Der nächste Schritt der ZSA umfasste die Analyse der Einlasszone hinsichtlich der Erfüllung der erstellten Richtlinien und möglicher Wechselwirkungen innerhalb der Zone sowie mit benachbarten Zonen, vgl. Tabelle 4.7. Dabei wurden sowohl der reguläre Betrieb als auch Fehlerfälle untersucht. Innerhalb der Einlasszone werden die Außenhülle, die Versteifungselemente, die Lärmminderungsmaßnahmen, das Eisschutzsystem und der Variationsmechanismus mit ihren zugehörigen Komponenten unterschieden, vgl. Abbildung 2.10. In Abhängigkeit der gewählten Umsetzungen der Teilsysteme können ähnliche Komponenten mehrfach vorhanden sein, wodurch sich Synergien zwischen den Teilsystemen ergeben können. Andererseits können auch Komponentenpaarungen mit Wechselwirkungen untereinander auftreten.

Tabelle 4.7 Ausgewählte Ergebnisse der Zonensicherheitsanalyse

\begin{tabular}{|c|c|c|c|}
\hline Komponenten & Mögliche Fehler & Mögliche Folgen & Vorkehrungen \\
\hline \multirow[t]{2}{*}{$\begin{array}{l}\text { Bewegliche } \\
\text { Komponenten }\end{array}$} & $\begin{array}{l}\text { Starke } \\
\text { Verschmutzung, } \\
\text { eindringende } \\
\text { Feuchtigkeit, } \\
\text { Vereisung, } \\
\text { Blockieren }\end{array}$ & $\begin{array}{l}\text { Verlust der } \\
\text { Variationsfähigkeit; } \\
\text { Schubverlust eines } \\
\text { Triebwerks }\end{array}$ & $\begin{array}{l}\text { Beschichtungen, } \\
\text { Ventilation, } \\
\text { Drainage, } \\
\text { Minimierung von } \\
\text { Lücken, } \\
\text { Eisschutzsystem }\end{array}$ \\
\hline & $\begin{array}{l}\text { Bauteilversagen und } \\
\text { Verlust durch } \\
\text { Vogelschlag, Feuer, } \\
\text { Druckluftleckage }\end{array}$ & $\begin{array}{l}\text { Beschädigung von } \\
\text { Fan oder Leitwerk; } \\
\text { Triebwerksschubver- } \\
\text { lust; } \\
\text { Flugzeugkontroll- } \\
\text { verlust }\end{array}$ & $\begin{array}{l}\text { Strukturelle } \\
\text { Dimensionierung, } \\
\text { Redundante } \\
\text { Absicherung gegen } \\
\text { Komponentenver- } \\
\text { lust }\end{array}$ \\
\hline
\end{tabular}


Tabelle 4.7 (Fortsetzung)

\begin{tabular}{l|l|l|l}
\hline Komponenten & Mögliche Fehler & Mögliche Folgen & Vorkehrungen \\
\hline Eisschutzsystem & Funktionsausfall & $\begin{array}{l}\text { Schubverlust eines } \\
\text { Triebwerks }\end{array}$ & $\begin{array}{l}\text { Enteisung per } \\
\text { Konturvariation }\end{array}$ \\
\hline $\begin{array}{l}\text { Kabel, Leitungen und } \\
\text { Rohre }\end{array}$ & $\begin{array}{l}\text { Erhöhte Belastungen } \\
\text { durch } \\
\text { Konturvariation; } \\
\text { Durchtrennen bei } \\
\text { Bauteilversagen }\end{array}$ & $\begin{array}{l}\text { Verlust der } \\
\text { Variationsfähigkeit; } \\
\text { Schubverlust eines } \\
\text { Triebwerks }\end{array}$ & $\begin{array}{l}\text { Flexible Gestaltung, } \\
\text { Mindestabstand } \\
\text { zueinander, ggf. } \\
\text { redundante } \\
\text { Ausführung }\end{array}$ \\
\hline $\begin{array}{l}\text { Heißlufteisschutzsys- } \\
\text { tem und hydraulische }\end{array}$ & $\begin{array}{l}\text { Leckage von } \\
\text { Hydraulikfluid kann } \\
\text { auf heiße } \\
\text { Oberflächen } \\
\text { auftreten und zu } \\
\text { Feuerentstehung } \\
\text { führen }\end{array}$ & $\begin{array}{l}\text { Schubverlust eines } \\
\text { Triebwerks; } \\
\text { Gefährliche } \\
\text { Ereignisse aufgrund } \\
\text { eines } \\
\text { unkontrollierten } \\
\text { Feuers }\end{array}$ & $\begin{array}{l}\text { Feuerdetektion und } \\
\text { Feuerlöschsystem, } \\
\text { Feuerschutzwände, } \\
\text { Ventilation, } \\
\text { Drainage, } \\
\text { Zündtemperatur } \\
\text { brennbarer Fluide } \\
>300\end{array}$ \\
\hline $\begin{array}{l}\text { Elektrische } \\
\text { Komponenten }\end{array}$ & $\begin{array}{l}\text { Schäden durch } \\
\text { Leckage von } \\
\text { Hydraulikfluid oder } \\
\text { Zapfluft }\end{array}$ & $\begin{array}{l}\text { Schubverlust eines } \\
\text { Triebwerks; } \\
\text { Gefährliche } \\
\text { Ereignisse aufgrund } \\
\text { eines } \\
\text { unkontrollierten } \\
\text { Feuers }\end{array}$ & $\begin{array}{l}\text { Überdruckaus- } \\
\text { gleichklappen, } \\
\text { Ventilation, } \\
\text { Drainage und } \\
\text { Hitzeschutz, } \\
\text { Schottwände }\end{array}$ \\
\cline { 2 - 4 } & $\begin{array}{l}\text { Schäden durch } \\
\text { Schmutz, Blitzschlag }\end{array}$ & $\begin{array}{l}\text { Schubverlust eines } \\
\text { Triebwerks }\end{array}$ & $\begin{array}{l}\text { Drainage, Erdung, } \\
\text { Schutz gegen } \\
\text { Wasser und Staub }\end{array}$ \\
\hline
\end{tabular}

\section{Analyse redundanzüberbrückender Fehler}

Zur Vermeidung voneinander abhängiger Fehlermöglichkeiten wurden mögliche gleichartige Fehler zu Beginn der CMA durch die Erstellung von Checklisten identifiziert. Tabelle 4.8 zeigt Beispiele für gleichartige Fehler variabler Einlässe auf, die während eines Fluges bei mehreren Triebwerken gleichzeitig möglich sind und somit gefährliche Ereignisse verursachen können. 
Tabelle 4.8 Ausgewählte Kategorien und Ursachen gleichartiger Fehler für variable Einlässe

\begin{tabular}{l|l|l|l}
\hline Art & Unterart & Ursache & Fehlerbeispiel \\
\hline $\begin{array}{l}\text { Konzeption und } \\
\text { Gestaltung }\end{array}$ & Systemarchitektur & $\begin{array}{l}\text { Gemeinsame } \\
\text { Schnittstellen } \\
\text { und Leitungen }\end{array}$ & $\begin{array}{l}\text { Fehlende Energie für } \\
\text { Eisschutzsystem oder } \\
\text { Variationsmechanismus } \\
\text { mehrerer Triebwerke }\end{array}$ \\
\cline { 2 - 4 } & & $\begin{array}{l}\text { Fehlender } \\
\text { Schutz von } \\
\text { Komponenten }\end{array}$ & $\begin{array}{l}\text { Systemausfall aufgrund } \\
\text { unzureichender } \\
\text { Anforderungsdefinition }\end{array}$ \\
\hline & Technologie & $\begin{array}{l}\text { Gemeinsame } \\
\text { Software }\end{array}$ & $\begin{array}{l}\text { Simultaner Ausfall des } \\
\text { Variationsmechanismus } \\
\text { in mehreren Triebwerken }\end{array}$ \\
\hline & & $\begin{array}{l}\text { Unerprobte, } \\
\text { anfällige } \\
\text { Technologie }\end{array}$ & $\begin{array}{l}\text { Allgemeine } \\
\text { Entwicklungsfehler, wie } \\
\text { unzureichende } \\
\text { Lebensdauer, durch } \\
\text { fehlende Erfahrung }\end{array}$ \\
\hline Herstellungsprozess & Hersteller & $\begin{array}{l}\text { Gemeinsamer } \\
\text { Hersteller }\end{array}$ & $\begin{array}{l}\text { Simultan auftretende } \\
\text { Fehler in mehreren } \\
\text { Triebwerken aufgrund } \\
\text { unzureichender } \\
\text { Personalschulung }\end{array}$ \\
\hline & & &
\end{tabular}

Diese identifizierten Fehler gilt es, mit Hilfe von Fail-Safe- oder Unabhängigkeitsprinzipen zu vermeiden bzw. deren Auswirkungen auf den Einlass eines einzelnen Triebwerks einzugrenzen. Mögliche Mittel hierfür sind Redundanz, Sicherungssysteme, Überwachungssysteme, Isolierung und Begrenzungen von Ausfalleffekten.

Beispielhafte Vorkehrungen, um Abhängigkeiten zwischen Fehlern variabler Einlässe mehrerer Triebwerke zu vermeiden, sind in Tabelle 4.9 aufgelistet. Es ist festzuhalten, dass durch diese Maßnahmen die Wahrscheinlichkeit des zeitgleichen Auftretens dieser Fehler in mehreren Triebwerken signifikant verringert, jedoch nicht vollständig ausgeschlossen werden kann. 
Tabelle 4.9 Vorkehrungen gegen Abhängigkeiten der Einlässe mehrerer Triebwerke

\begin{tabular}{l|l|l}
\hline Unterart & Fehler & Vorkehrungen \\
\hline Systemarchitektur & $\begin{array}{l}\text { Lokale Ereignisse beeinträchtigen } \\
\text { Pfade vom zentralen } \\
\text { Flugzeugkontrollsystem zu den } \\
\text { Einlässen und verhindern } \\
\text { Variation mehrerer Einlässe }\end{array}$ & $\begin{array}{l}\text { Räumliche, mechanische und } \\
\text { elektrische Trennung der } \\
\text { Leitungen und Kabel zu den } \\
\text { variablen Einlässen } \\
\text { verschiedener Triebwerke }\end{array}$ \\
\hline Technologie & $\begin{array}{l}\text { Allgemeine Entwicklungsfehler, } \\
\text { wie zu geringe Lebensdauer, } \\
\text { durch fehlende Erfahrung }\end{array}$ & $\begin{array}{l}\text { Wenn möglich, Verwendung } \\
\text { erprobter Technologien; } \\
\text { ausreichende Anzahl Tests }\end{array}$ \\
\hline Hersteller & $\begin{array}{l}\text { Fehlerhafte Montage von } \\
\text { Komponenten, wodurch mehrere } \\
\text { Einlässe beeinträchtigt werden }\end{array}$ & $\begin{array}{l}\text { Verschiedene Hersteller wählen; } \\
\text { Prozess- und } \\
\text { Qualitätsüberwachung; in einem } \\
\text { Flugzeug nur Einlässe } \\
\text { unterschiedlicher Chargen } \\
\text { verwenden }\end{array}$ \\
\hline Umwelteinflüsse & $\begin{array}{l}\text { Vogelschlag auf mehrere } \\
\text { Triebwerke }\end{array}$ & $\begin{array}{l}\text { Rückführung in } \\
\text { versagenssichere Geometrie; } \\
\text { Vogelvergrämungsmaßnahmen } \\
\text { am Flughafen }\end{array}$ \\
\hline
\end{tabular}

\section{Erkenntnisse aus der Analyse von Fehlern gemeinsamer Ursachen}

Die Ergebnisse der CCA untermauern die Bedeutung der Auswahl des Eisschutzund des Stellsystems variabler Einlässe sowie den Schutz vor äußeren Einflüssen, wie Vogel- und Blitzschlag. Darüber hinaus sind Fehlerabhängigkeiten von Einlässen mehrerer Triebwerke eines Flugzeugs zu vermeiden. Hierfür eignet sich insbesondere die Nutzung von Redundanz.

Für die Konzeptgruppe, die verschiebbare starre Segmente für die Konturvariation verwendet, ist eine Erweiterung der Oberfläche, die vor Vereisung zu schützen ist, erforderlich. Dadurch wird Eisbildung zwischen den Segmenten verhindert. Infolgedessen erfordert der Vereisungsschutz dieser Konzeptgruppe mehr Energie.

Weiterhin sollte das ausgewählte Eisschutzsystem keine potenziellen Wechselwirkungen mit dem verwendeten Variationsmechanismus des jeweiligen Konzepts sowie, falls vorhanden, dem verformbaren Oberflächenmaterial aufweisen. $\mathrm{Zu}$ diesen Wechselwirkungen zählen beispielsweise Leckagen von Fluiden, die eine Feuerentstehung begünstigen oder elektrische Komponenten beschädigen können. Darüber hinaus sind erhöhte Beanspruchungen von Kabeln und Leitungen bei der Konturvariation der betreffenden Konzeptgruppen durch eine flexible Gestaltung 
und einen Mindestabstand zwischen den Komponenten zu vermeiden. Andererseits könnte die Konturvariation dieser Konzeptgruppen auch als sekundäres Eisschutzsystem genutzt werden. So lange zusätzliche Fehlerabhängigkeiten verhindert werden, könnten zudem Synergieeffekte zwischen einem Stellsystem und einem Eisschutzsystem mit gleicher Art der Energieversorgung genutzt werden. Diese bestehen beispielsweise in der gemeinsamen Nutzung von Komponenten und können zu einer Massenreduktion beitragen.

Bei der Konzeptgruppe der aerodynamischen Grenzschichtbeeinflussung sind vor allem die Absaug- bzw. Einblasschlitze vor äußeren Einflüssen, wie dem Verstopfen infolge von Vereisung oder Verschmutzung, zu schützen.

\subsubsection{Integrationsstudien}

Der Auswahl geeigneter Teilsysteme für das Einstellen der Geometrie, für die Reduzierung des Triebwerkslärms und für den Vereisungsschutz fällt eine große Bedeutung zu. Diese Auswahl bestimmt die Funktionalität, die Zuverlässigkeit, die Kosten und die Sicherheit der Konzeptgruppen maßgeblich.

\section{Stellsystem}

Für die Antriebssysteme der Konzepte, die die Geometrie variieren, indem sie starre Komponenten verschieben oder das Oberflächenmaterial des Einlasses elastisch verformen, werden vorrangig Aktoren angedacht, die eine axiale Bewegung realisieren. Hierfür können als konventionelle Lösungen elektrische, hydraulische oder pneumatische Aktoren ausgewählt werden, vgl. Abschnitt 2.4.3. Aufgrund der überwiegenden Nachteile bezüglich Masse und Anfälligkeit für Leckagen hydraulischer und pneumatischer Aktoren gegenüber elektromechanischen Lösungen, fällt die Auswahl der Aktorik auf die letztgenannte Antriebsart.

Unter den elektromechanischen Aktoren stellen Linearsysteme mit Kugelgewindetrieb, bestehend aus Motor, Kupplung, Lagerung und Kugelgewindetrieb, eine gut geeignete Lösung dar [302, S. 579]. Sie vereinen eine große Vorschubkraft mit hoher Stellgenauigkeit und Stellgeschwindigkeit, sind wartungsarm und weisen akzeptable Anschaffungskosten auf [302, S. 579].

Für Konzepte, die die Strömungsgrenzschicht beeinflussen, ist ebenfalls ein Stellsystem zum Öffnen und Schließen der Absaug- bzw. Einblasschlitze erforderlich. Hierbei werden elektrisch angesteuerte Magnetventile ausgewählt. Magnetventile sind technisch erprobt und kommen auch in regelungstechnischen Anwendungen in der Luftfahrt zum Einsatz [10, S. 1668-1669]. Vom Einsatz unkonventioneller Aktoren, vgl. Abschnitt 2.4.3, wird bei allen Konzeptgruppen aus Gründen der Zuverlässigkeit abgesehen. 


\section{Lärmminderungsmaßnahmen}

Herkömmliche Akustikauskleidungen stellen eine bewährte Lärmminderungsmaßnahme dar. Die Funktionsfähigkeit und Lebensdauer flexibler Akustikauskleidungen ist noch nicht umfassend nachgewiesen. Auch das Prinzip der Schallauslöschung durch Gegenschall ist noch unzureichend erprobt, sodass für alle Konzeptgruppen konventionelle starre Akustikauskleidungen eingeplant werden.

Bei der Umsetzung in der Konzeptgruppe, die starre Segmente verschiebt, ist $\mathrm{zu}$ beachten, dass auch die Akustikauskleidungen segmentiert werden müssen. Zudem dürfen sie den Variationsmechanismus nicht einschränken, beispielsweise indem sie eine Überlappung benachbarter Segmente behindern. Dadurch wird der von Akustikauskleidungen abdeckbare Anteil der Einlassoberfläche verringert.

Bei den grenzschichtbeeinflussenden Konzepten ist zu beachten, dass der Bauraum ebenfalls aufgrund der voraussichtlich kleineren Konturabmaße sowie den zu integrierenden Einblas- bzw. Absaugschlitzen eingeschränkt ist.

Für die Gruppe der elastischen Oberflächenverformung müssten die starren Akustikauskleidungen stark segmentiert werden, um die Einschränkung der Oberflächenverformung zu minimieren. Alternativ könnte auch ein starrer Bereich im Diffusor beibehalten werden, um herkömmliche Auskleidungen zu integrieren. Der vollständige Verzicht auf Akustikauskleidungen würde eine inakzeptable Erhöhung der Lärmemissionen nach sich ziehen.

\section{Schutz vor Vereisung}

Die Eiserkennung kann uneingeschränkt weiterhin über die erprobten elektromechanischen Eisdetektoren erfolgen. Für die Auswahl der geeigneten Eisschutzsysteme (IPSs) für die jeweiligen Konzeptgruppen wurde eine gewichtete Punktbewertung der in Abschnitt 2.3.2 vorgestellten Eisschutzsystemarten durchgeführt. Diese Bewertung basiert auf der Erfüllung der Bewertungskriterien für variable Einlässe, vgl. Abschnitt 4.1.1. Zusätzlich kommen zu diesen Bewertungskriterien noch spezifische Ergänzungen für IPSs hinzu.

Einige IPS-Arten können die aerodynamische Funktionalität des variablen Einlasses durch potenzielle zusätzliche Stufen, Spalte oder Flüssigkeiten auf der Oberfläche beeinträchtigen. Die Integrierbarkeit eines IPS wird durch den erforderlichen Bauraum sowie mögliche Synergien und Interaktionen mit anderen Komponenten in der Einlasszone beeinflusst. Die Masse des IPS wird durch die strukturell erforderlichen Dimensionen, Betriebsmittel sowie zusätzlich erforderliche Komponenten, wie Druckausgleichklappen, bestimmt. Die Wahl des IPS beeinflusst zudem die Sicherheit, falls die Entstehung von kleineren Eisansammlungen sowie deren Abplatzen nicht auszuschließen ist. Dadurch könnten 
einerseits Strömungsablösungen und andererseits Beschädigungen des Fans auftreten. Auch steigt bei der Verwendung bestimmter Kombinationen von IPSs und Stellsystemen die Feuergefahr. Die Zuverlässigkeit eines IPS wird durch seine Komplexität, die Anfälligkeit gegenüber Erosion oder Verstopfen erforderlicher Öffnungen durch Fremdkörper sowie seinen Lebensdauereinfluss auf andere Einlasskomponenten bestimmt. Die Lebenswegkosten werden durch den Energiebedarf des IPS sowie erforderliche Betriebsmittel bestimmt. Die Entwicklungskosten sind vorrangig abhängig von den existierenden Erfahrungswerten bezüglich des IPS und den erforderlichen Zulassungsnachweisen, während die Produktionskosten durch innovative Materialien, aufwändige Herstellungsmethoden und eine erforderliche Qualitätssicherung negativ beeinflusst werden können.

Die gewichtete Punktbewertung erfordert neben den relativen Gewichtungsfaktoren $w_{i}$ der jeweiligen Kriterien, vgl. Abschnitt 4.1.4, auch numerische Werte für die Erfüllung eines Kriteriums durch eine Lösungsvariante $m_{i, j}$, vgl. Abschnitt 3.2.4. Diese Werte in einem Bereich von sehr schlecht (0 oder - -) bis sehr gut $(4$ oder ++ ) wurden durch eine Gruppe von Ingenieuren vergeben. Dabei basiert die Bewertung auf der Fachliteratur [78], [112], [114], [118], Herstellerangaben [134] sowie den zuvor durchgeführten Analysen. Zudem wurde die Bewertung der IPS-Optionen für die jeweiligen Konzeptgruppen separat durchgeführt. Dadurch konnten die jeweiligen Charakteristiken, die in unterschiedlichen Synergien und Wechselwirkungen resultieren, berücksichtigt werden.

Die Hauptcharakteristiken der Konzeptgruppe, die die Einlassgeometrie durch Verschieben starrer Segmente variiert, bestehen in der eingesetzten elektrischen Aktorik und der notwendigen Erweiterung des IPS auf den Bereich zwischen einzelnen Segmenten.

Für diese Konzeptgruppe bieten Heißluft-IPSs die höchste aerodynamische Effizienz, da sie durch den Eisverhütungsbetrieb die bestmögliche Oberfläche gewährleisten. Elektrothermische IPSs ermöglichen sowohl im Eisverhütungsbetrieb als auch im Nassfließbetrieb eine sehr gute Oberfläche. Jedoch verbleibt im Nassfließbetrieb das Risiko der Bildung von Runback-Eis [113], welches wiederum durch die Erweiterung der zu schützenden Fläche reduziert wird. Flüssigkeits-IPSs ermöglichen ebenfalls den Nassfließbetrieb, verursachen allerdings eine geringe Widerstandserhöhung [134]. Diese begründet sich mit dem strömungsmechanischen Einfluss des Enteisungsfluids und der mikroporösen Löcher. Zudem ist kein kontinuierlicher Betrieb möglich, was zu geringfügigen Eisansätzen führen kann. Elektromechanische und Druckluft-IPSs ermöglichen ausschließlich einen Enteisungsbetrieb mit allen daraus resultierenden Nachteilen, z. B. mögliches Resteis. Während der aerodynamische Einfluss der schwingenden Oberfläche von elektromechanischen IPSs vernachlässigbar ist, führen 
die aufblasbaren Druckzellen der Druckluft-IPSs zudem zu einer messbaren Widerstandserhöhung [134].

Im Vergleich zu den Alternativen, lassen sich elektrische IPSs deutlich leichter in den Einlass integrieren, da sie den geringsten Bauraum erfordern und potenziell viele Synergieeffekte mit dem bevorzugten elektrischen Aktorsystem bieten. Beispielsweise könnten Teile der erforderlichen Leitungen, der wasserdichten Komponentengehäuse, der Drainage, der Erdung und des Blitzschlagschutzes von beiden Systemen genutzt werden [53], [57], [299]. Da elektrothermische IPSs weniger Bauraum benötigen und gleichzeitig einen geringeren Einfluss auf die Materialwahl der Einlassoberfläche haben als elektromechanische IPSs, werden sie hinsichtlich der Integrierbarkeit als eine sehr gute Option angesehen. Flüssigkeits-IPSs benötigen hingegen viel Bauraum für einen Flüssigkeitstank, Rohre und Ventile. Darüber hinaus sind Teleskoprohre oder flexible Kupplungen erforderlich, um das Enteisungsfluid für alle einstellbaren Geometrien zur Außenhülle zu transportieren. Wechselwirkungen zwischen dem elektrischen Aktorsystem und dem Enteisungsfluid werden im Fall möglicher Leckagen durch die bereits erforderlichen wasserdichten Komponentengehäuse des Aktorsystems vermieden. Pneumatische IPSs erfordern einen großen Bauraum für die Integration flexibler Leitungen für den Lufttransport sowie Überdruckklappen, um mögliche Wechselwirkungen mit dem Stellsystem zu vermeiden. Darüber hinaus benötigt das Heißluft-IPS ein komplexes Zapfluftverteilungs- und Regelsystem sowie einen Überhitzungsschutz für das Stellsystem [113], [299]. Für die Anwendung eines Druckluft-IPS ist hingegen die komplexe Integration der aufblasbaren Druckzellen auf den beweglichen Außensegmenten erforderlich. Zudem ermöglicht dieses System keine Enteisung zwischen den Segmenten.

Die Komponenten elektrischer IPSs weisen nur eine geringe Masse auf [112], [134]. Durch Synergien mit dem Stellsystem könnte diese Masse zusätzlich verringert werden. Aufgrund der erforderlichen Rohre weisen pneumatische IPSs eine deutlich höhere Masse auf, die vom Flüssigkeits-IPS noch übertroffen wird. Zudem erfordern Druckluft-IPSs Pumpen zum Aufblasen und Absaugen der Druckzellen, Heißluft-IPSs Schottwände als Überhitzungsschutz und Flüssigkeits-IPSs das Enteisungsfluid und den Vorratstank [134].

Elektrothermische IPSs werden als sicherste Option für die vorliegende Konzeptgruppe erachtet, da sie durch den Eisverhütungsbetrieb das Risiko einer Strömungsablösung vermeiden. Weiterhin wird die zusätzliche Feuergefahr durch die bereits für das elektrische Stellsystem erforderlichen Feuerverhütungsmaßnahmen, wie Erdung, minimiert. Heißluft-IPSs vermeiden ebenfalls das Risiko einer Strömungsablösung, jedoch bergen sie im Fall einer Leckage zusätzliche Risiken für Beschädigungen durch Überhitzung und Druckluft. Diesen Risiken kann 
durch konstruktive Vorkehrungen, wie Schottwänden, Ventilation und Druckausgleichklappen, begegnet werden [53], [57]. Flüssigkeits-IPSs sind anfällig für Strömungsablösungen aufgrund von Eisansammlungen, falls der Vorratstank für das Enteisungsfluid durch Leckagen oder anhaltende Eisbildungsbedingungen entleert ist. Bei elektromechanischen und Druckluft-IPSs können Strömungsablösungen aufgrund von Resteis auftreten, da diese IPSs ausschließlich für den Enteisungsbetrieb geeignet sind. Darüber hinaus kann die Form der aufblasbaren Druckzellen des Druckluft-IPS zu Strömungsbeeinflussungen führen [34].

Heißluft-IPSs bieten abgesehen von den erforderlichen flexiblen Leitungen eine lange Lebensdauer und hohe Zuverlässigkeit [134]. Bei diesen Systemen ist der Einfluss des erforderlichen Zapfluftmassenstroms auf die Triebwerksleistung zu berücksichtigen. Druckluft-IPSs haben eine Lebensdauer von wenigen Monaten bis hin zu 5 Jahren, wodurch erhöhter Wartungsaufwand entsteht [134]. Dies begründet sich vorrangig aus der Anfälligkeit der Druckzellen gegenüber äußeren Einflüssen, wie Sand, Hagel oder Vogelschlag, sowie aus der begrenzten Lebensdauer der flexiblen Leitungen. Zudem muss der Einfluss der erforderlichen Druckluft und Steuerungsenergie auf die Betriebskosten berücksichtigt werden [134]. Flüssigkeit-IPSs ermöglichen, abgesehen von den notwendigen flexiblen Leitungen, eine relativ lange Lebensdauer [134]. Allerdings könnten die mikroporösen Löcher durch Fremdkörper verstopfen. Zudem resultiert das erforderliche Enteisungsfluid in einer verringerten Nutzlast und erhöhten Betriebskosten [134]. Elektrothermische IPSs bieten eine lange Lebensdauer auf Kosten eines hohen Leistungsbedarfs im Eisverhütungsbetrieb [134]. Dieser Leistungsbedarf kann durch die Verwendung im Enteisungsbetrieb erheblich reduziert werden. Elektromechanische IPSs vereinen einen sehr niedrigen Leistungsbedarf mit einer langen Komponentenlebensdauer [134]. Jedoch ist der mögliche Schwingungseinfluss auf die Lebensdauer des Einlassoberflächenmaterials zu beachten.

Alle untersuchten IPSs weisen nahezu ähnliche Herstellungskosten auf [134]. Sie unterscheiden sich jedoch hinsichtlich des erforderlichen Entwicklungsaufwands. Elektrothermische IPSs wurden bereits in Einlässen eingesetzt und sind die bevorzugte Option für komplexe dreidimensionale Einlasstypen [113]. Auch Heißluft-IPSs sind sehr erprobt in der Anwendung in Einlässen. Druckluft-, Flüssigkeits- und elektromechanische IPSs fanden bisher keine Anwendung in Triebwerkseinlässen. Dies würde bei einer Anwendung in variablen Einlässen den erforderlichen Simulations- und Testaufwand während der Entwicklung und Zulassung erhöhen.

Die Ergebnisse der Bewertung der Konzeptgruppe verschiebbarer Segmente sind in Tabelle 4.10 zusammengefasst. Die Netzdiagramme in Abbildung 4.16 
verdeutlichen die sehr gute Eignung von elektrothermischen und von HeißluftIPSs für diese Konzeptgruppe. Die relativen Gewichtungsfaktoren $w_{i}$ der Kriterien werden bei der relativen Bewertung in Tabelle 4.13 berücksichtigt.

Tabelle 4.10 Eisschutzsystembewertung der Konzeptgruppe verschiebbarer Segmente

\begin{tabular}{l|l|l|l|l|l}
\hline Kriterium & Heißluft & Druckluft & Flüssigkeit & $\begin{array}{l}\text { Elektro- } \\
\text { thermisch }\end{array}$ & $\begin{array}{l}\text { Elektro- } \\
\text { mechanisch }\end{array}$ \\
\hline $\begin{array}{l}\text { Aerodynamische } \\
\text { Funktionalität } \\
\left(\boldsymbol{w}_{1}=0,267\right)\end{array}$ & ++ & -- & $\mathrm{o}$ & + & - \\
\hline $\begin{array}{l}\text { Integrierbarkeit } \\
\left(\boldsymbol{w}_{2}=0,067\right)\end{array}$ & - & - & 0 & ++ & + \\
\hline $\begin{array}{l}\text { Masse und Beanspru- } \\
\text { chungswiderstand } \\
\left(\boldsymbol{w}_{3}=0,133\right)\end{array}$ & $\mathrm{o}$ & $\mathrm{o}$ & -- & + & ++ \\
\hline $\begin{array}{l}\text { Sicherheit } \\
\left(\boldsymbol{w}_{4}=0,267\right)\end{array}$ & + & - & $\mathrm{o}$ & ++ & $\mathrm{o}$ \\
\hline $\begin{array}{l}\text { Zuverlässigkeit und } \\
\text { Lebenswegkosten } \\
\left(\boldsymbol{w}_{5}=0,200\right)\end{array}$ & + & -- & $\mathrm{o}$ & + & + \\
$\begin{array}{l}\text { Entwicklungs- und } \\
\text { Produktionskosten } \\
\left(\boldsymbol{w}_{6}=0,067\right)\end{array}$ & + & $\mathrm{o}$ & $\mathrm{o}$ & ++ & $\mathrm{o}$ \\
\hline
\end{tabular}

Zellwerte: Erfüllung des Kriteriums ist sehr gut ++, gut +, durchschnittlich o, schlecht oder sehr schlecht --

Die Hauptcharakteristik der nächsten Konzeptgruppe besteht in der Verwendung eines elastischen Oberflächenmaterials, das mit Hilfe eines Stellsystems verformt wird. Das zu verwendende IPS muss bei dieser Konzeptgruppe eine vergleichbare Fläche, wie bei der Verwendung starrer Einlässe, abdecken. Das verformbare Oberflächenmaterial darf dabei jedoch nicht durch hohe Temperaturen oder starke Verformungen beeinträchtigt werden. Der Einfluss der jeweiligen IPS-Varianten auf die aerodynamische Funktionalität, die Masse sowie die Entwicklungs- und Produktionskosten verhält sich ähnlich wie bei der Konzeptgruppe verschiebbarer starrer Komponenten.

Die Integrierbarkeit von Druckluft-IPSs wird bei der vorliegenden Einlasskonzeptgruppe erschwert, da zusätzliche Wechselwirkungen zwischen den aufblasbaren Druckzellen und dem elastischen Oberflächenmaterial auftreten können. Der 


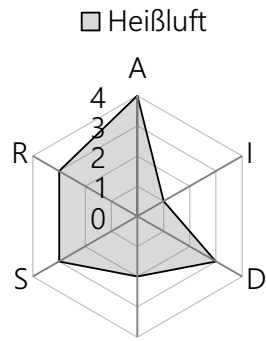

W

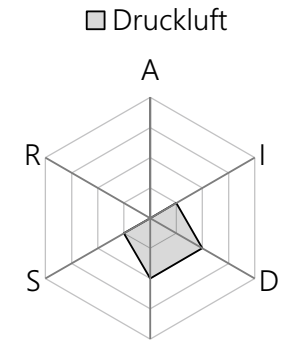

W $\square$ Flüssigkeit

A

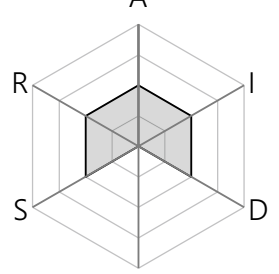

W
Elektrothermisch

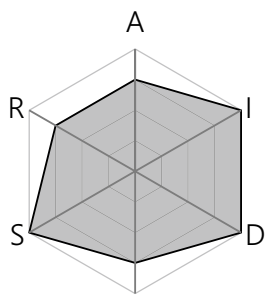

W
Elektromechanisch

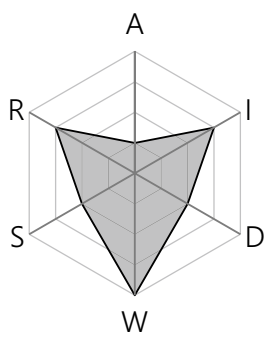

Aerodynamische Funktionalität (A)

Integrierbarkeit (I)

Zuverlässigkeit und Lebenswegkosten (R)

Sicherheit (S)

Entwicklungs- und Produktionskosten (D)

Masse und Beanspruchungswiderstand (W)

Abbildung 4.16 Eisschutzsystembewertung der Konzeptgruppe verschiebbarer Segmente

Einfluss der anderen IPS-Varianten auf die Integrierbarkeit ist vergleichbar mit dem Einfluss auf die vorherige Konzeptgruppe.

Heißluft- und elektrothermische IPSs erwärmen die Außenhülle des Einlasses. Die daraus resultierenden Temperaturänderungen können sich negativ auf die Lebensdauer des verformbaren Oberflächenmaterials auswirken und zu reduzierten Wartungszyklen führen. Darüber hinaus könnten Fehler dieser IPSs zu Überhitzungen führen, die potenziell zu Feuer oder strukturellem Versagen und somit zu gefährlichen Ereignissen führen können. Die übrigen IPS-Varianten haben keinen signifikanten Temperatureinfluss auf das Oberflächenmaterial, weshalb die Bewertung von Sicherheit sowie Zuverlässigkeit und Lebenswegkosten mit der der vorherigen Konzeptgruppe übereinstimmt. 
Die Bewertung hebt die gute Eignung von elektrischen und Heißluft-IPSs für diese Konzeptgruppe hervor, vgl. Tabelle 4.11 und Abbildung 4.17.

Tabelle 4.11 Eisschutzsystembewertung der Konzeptgruppe verformbarer Oberflächen

\begin{tabular}{l|l|l|l|l|l}
\hline Kriterium & Heißluft & Druckluft & Flüssigkeit & $\begin{array}{l}\text { Elektro- } \\
\text { thermisch }\end{array}$ & $\begin{array}{l}\text { Elektro- } \\
\text { mechanisch }\end{array}$ \\
\hline $\begin{array}{l}\text { Aerodynamische } \\
\text { Funktionalität } \\
\left(\boldsymbol{w}_{1}=0,267\right)\end{array}$ & ++ & -- & $\mathrm{o}$ & + & - \\
\hline $\begin{array}{l}\text { Integrierbarkeit } \\
\left(\boldsymbol{w}_{2}=0,067\right)\end{array}$ & - & -- & 0 & ++ & + \\
\hline $\begin{array}{l}\text { Masse und Beanspru- } \\
\text { chungswiderstand } \\
\left(\boldsymbol{w}_{3}=0,133\right)\end{array}$ & $\mathrm{o}$ & $\mathrm{o}$ & -- & + & ++ \\
\hline $\begin{array}{l}\text { Sicherheit } \\
\left(\boldsymbol{w}_{4}=0,267\right)\end{array}$ & - & - & $\mathrm{o}$ & $\mathrm{o}$ & $\mathrm{o}$ \\
\hline $\begin{array}{l}\text { Zuverlässigkeit und } \\
\text { Lebenswegkosten } \\
\left(\boldsymbol{w}_{5}=0,200\right)\end{array}$ & $\mathrm{o}$ & -- & $\mathrm{o}$ & $\mathrm{o}$ & + \\
\hline $\begin{array}{l}\text { Entwicklungs- und } \\
\text { Produktionskosten } \\
\left(\boldsymbol{w}_{6}=0,067\right)\end{array}$ & + & $\mathrm{o}$ & $\mathrm{o}$ & ++ & $\mathrm{o}$ \\
\hline
\end{tabular}

Zellwerte: Erfüllung des Kriteriums ist sehr gut ++, gut +, durchschnittlich o, schlecht oder sehr schlecht --

Die Konzeptgruppe der Grenzschichtbeeinflussung wird durch eine starre Einlasskontur mit zahlreichen über den Einlass verteilten Lufteinblas- bzw. Luftabsaugschlitzen charakterisiert. Diese Schlitze sind, ebenso wie der Bereich der Einlasslippe, vor Vereisung zu schützen.

Die aerodynamische Funktionalität dieser Konzeptgruppe kann bei einem Einsatz von Druckluft- und Flüssigkeits-IPSs erheblich beeinträchtigt werden. Grund hierfür sind mögliche Interaktionen der aufblasbaren Druckzellen bzw. des Enteisungsfluids mit den Absaug- bzw. Einblasschlitzen des IPS und der Strömungsgrenzschicht. Heißluft-IPSs und elektrische IPSs haben keinen Einfluss auf die aerodynamische Funktionsfähigkeit dieser Konzeptgruppe und werden wie bei der ersten Konzeptgruppe bewertet.

Die Integration von Heißluft-IPSs in Einlasskonzepte mit Grenzschichtbeeinflussung bietet potenzielle Vorteile und Synergien durch die Nutzung gemeinsamer Komponenten für den Transport der erforderlichen Luft. Darüber hinaus 


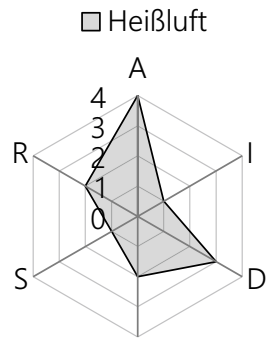

W

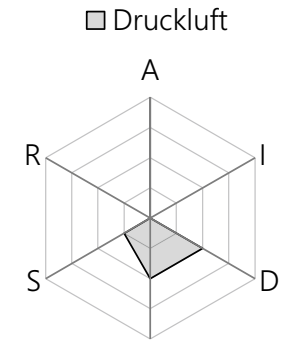

W $\square$ Flüssigkeit

A

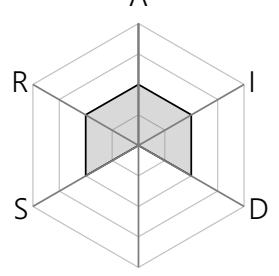

W
Elektrothermisch

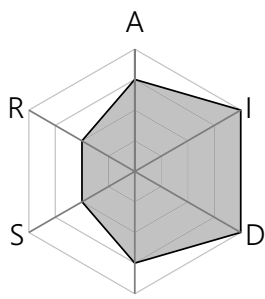

W
Elektromechanisch

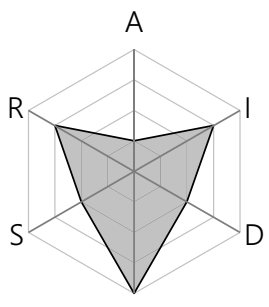

W

Aerodynamische Funktionalität (A)

Integrierbarkeit (I)

Zuverlässigkeit und Lebenswegkosten (R)

Sicherheit (S)

Entwicklungs- und Produktionskosten (D)

Masse und Beanspruchungswiderstand (W)

Abbildung 4.17 Eisschutzsystembewertung der Konzeptgruppe verformbarer Oberflächen

sind keine flexiblen Leitungen erforderlich, da ein starrer Einlass verwendet wird. Druckluft-IPSs könnten ähnliche Synergien nutzen. Allerdings überwiegt hierbei der erforderliche Konstruktionsaufwand zur Minderung von Interaktionen der Druckzellen mit den Schlitzen. Während die Integrierbarkeit der Leitungen für das Flüssigkeits-IPS vereinfacht ist, erfordert die Vermeidung von Wechselwirkungen mit der Grenzschicht und den Schlitzen einen erhöhten Konstruktionsaufwand. Die Integration von elektrischen IPSs ist für diese Konzeptgruppe aufwendiger. Zum einen sind die nutzbaren Synergien mit dem Stellsystem stark eingeschränkt. Zum anderen sind Interaktionen zwischen Elektronik und Luftkanälen $\mathrm{zu}$ vermeiden.

Die Masse pneumatischer IPS-Varianten kann aufgrund der möglichen Synergieeffekte deutlich reduziert werden, während die Masse elektrischer IPSs durch 
die Maßnahmen zur Vermeidung von Interaktionen mit dem Grenzschichtbeeinflussungssystem leicht erhöht wird. Flüssigkeits-IPS stellen weiterhin die Variante mit der höchsten Masse dar.

Bei der Verwendung von Heißluft-IPSs wird die Schadens- und Feuergefahr erheblich verringert, da keine elektrische Aktorik vorhanden ist. Dies führt zu einer erhöhten Sicherheit. Die potenziellen Wechselwirkungen zwischen Druckluft- bzw. Flüssigkeits-IPSs und der aerodynamischen Geometrie dieser Konzeptgruppe können zu gefährlichen Strömungsablösungen führen. Die Sicherheit aller elektrischen IPSs wird nur unwesentlich beeinträchtigt.

Der mögliche Verzicht auf flexible Leitungen infolge der starren Kontur erhöht die Zuverlässigkeit von pneumatischen und Flüssigkeits-IPSs. Die Lebensdauer von Druckluft-IPSs ist jedoch weiterhin unzureichend. Elektrische IPSs benötigen ebenfalls keine flexible Verkabelung mehr, was ihre Zuverlässigkeit und Lebenszykluskosten nur geringfügig verbessert.

Die Entwicklungs- und Produktionskosten der jeweiligen IPS-Varianten dieser Konzeptgruppe ähneln denen der Konzeptgruppe zur Einlassvariation durch Verschieben starrer Komponenten.

Aus der Bewertung der IPS-Varianten für die Konzeptgruppe der Grenzschichtbeeinflussung geht eine sehr gute Eignung von elektrothermischen und Heißluft-IPSs hervor, vgl. Tabelle 4.12 und Abbildung 4.18.

Der relative Gewichtungsfaktor $w_{i}$ eines Kriteriums $i$ fließt in die relative Bewertung $r_{j}$ einer IPS-Variante $j$ mit ein, vgl. Gleichung (3.2). Diese Bewertung geht aus Tabelle 4.13 hervor, wobei die ausgewählten Kombinationen fett hervorgehoben sind. Die relative Bewertung $r_{j}$ entspricht dabei nicht zwingend der abschließenden Auswahl, sondern unterstützt diese quantitativ.

Für die Konzeptgruppe des Verschiebens starrer Komponenten sollte im weiteren Verlauf der Entwicklung die Anwendung elektrothermischer IPSs verfolgt werden. Diese weisen gegenüber der ersten Alternative in Form der HeißluftIPSs Vorteile bezüglich eines akzeptablen Leistungsbedarfs in Verbindung mit einer größeren vor Eis zu schützenden Oberfläche auf. Zudem ermöglichen sie die Nutzung zahlreicher Synergien und sind anwendungserprobt in komplexen dreidimensionalen Einlässen. Der Nachteil, dass elektrothermische IPSs nur im Nassfließbetrieb, der die Bildung von Runback-Eis ermöglicht, energieeffizient arbeiten, wird durch die erforderliche Erweiterung der zu schützenden Oberfläche hinfällig. Theoretisch kann sich hinter dem Fan zwar weiterhin Runback-Eis bilden. Die davon ausgehende Gefahr für andere Triebwerkskomponenten ist jedoch deutlich verringert.

Bei der Konzeptgruppe der elastischen Oberflächenverformung können neben elektrothermischen und Heißluft-IPSs auch elektromechanische IPSs in Betracht 
Tabelle 4.12 Eisschutzsystembewertung der Konzeptgruppe zur Grenzschichtbeeinflussung

\begin{tabular}{|c|c|c|c|c|c|}
\hline Kriterium & Heißluft & Druckluft & Flüssigkeit & $\begin{array}{l}\text { Elektro- } \\
\text { thermisch }\end{array}$ & $\begin{array}{l}\text { Elektro- } \\
\text { mechanisch }\end{array}$ \\
\hline $\begin{array}{l}\text { Aerodynamische } \\
\text { Funktionalität } \\
\left(\boldsymbol{w}_{1}=0,267\right)\end{array}$ & ++ & -- & - & + & - \\
\hline $\begin{array}{l}\text { Integrierbarkeit } \\
\left(\boldsymbol{w}_{2}=0,067\right)\end{array}$ & ++ & -- & o & + & o \\
\hline $\begin{array}{l}\text { Masse und Beanspru- } \\
\text { chungswiderstand } \\
\left(\boldsymbol{w}_{3}=0,133\right)\end{array}$ & + & + & -- & + & + \\
\hline $\begin{array}{l}\text { Sicherheit } \\
\left(\boldsymbol{w}_{4}=0,267\right)\end{array}$ & ++ & -- & - & ++ & o \\
\hline $\begin{array}{l}\text { Zuverlässigkeit und } \\
\text { Lebenswegkosten } \\
\left(\boldsymbol{w}_{5}=0,200\right)\end{array}$ & ++ & -- & + & + & + \\
\hline $\begin{array}{l}\text { Entwicklungs- und } \\
\text { Produktionskosten } \\
\left(\boldsymbol{w}_{6}=0,067\right)\end{array}$ & + & o & o & ++ & o \\
\hline \multicolumn{6}{|c|}{$\begin{array}{l}\text { Zellwerte: Erfüllung des Kriteriums ist sehr gut ++, gut }+ \text {, durchschnittlich o, schlecht }- \\
\text { oder sehr schlecht }--\end{array}$} \\
\hline
\end{tabular}

gezogen werden. Falls durch Versuche nachgewiesen werden kann, dass elektromechanische IPSs keine negativen Auswirkungen auf die Aerodynamik und die Materiallebensdauer haben, würde diese IPS-Variante die beste Eignung aufweisen. Die Hauptgründe dafür sind die möglichen Synergien mit dem Stellsystem sowie die Vermeidung eines Temperatureinflusses auf das elastische Oberflächenmaterial.

Grenzschichtbeeinflussende Konzepte verwenden Einblas- bzw. Absaugschlitze anstatt eines Stellsystems. Diese Schlitze müssen vor Eis und Schmutz geschützt werden, wofür Druckluft verwendet werden könnte. Hierbei muss die Lufttemperatur geregelt werden, um Schäden am Verdichtersystem zu vermeiden. Der Einsatz eines konventionellen Heißluft-IPS erscheint bei dieser Konzeptgruppe aufgrund der möglichen Synergieeffekte, beispielsweise durch Nutzung gemeinsamer Leitungen, und der wegfallenden Wechselwirkungen mit einem Stellsystem ideal. 


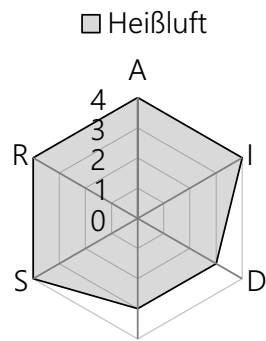

W

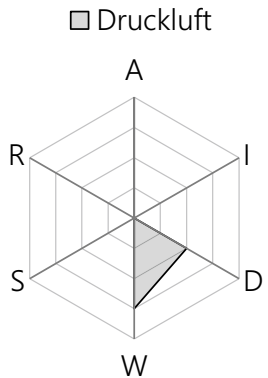

W $\square$ Flüssigkeit

A

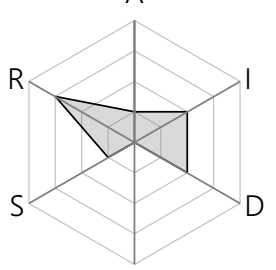

W
Elektrothermisch

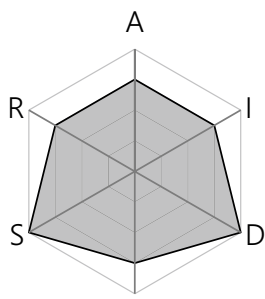

W
Elektromechanisch

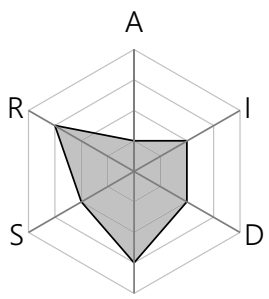

W

Aerodynamische Funktionalität (A)

Integrierbarkeit (I)

Zuverlässigkeit und Lebenswegkosten (R)

Entwicklungs- und Produktionskosten (D)

Sicherheit (S)

Masse und Beanspruchungswiderstand (W)

Abbildung 4.18 Eisschutzsystembewertung der Konzeptgruppe zur Grenzschichtbeeinflussung

Tabelle 4.13 Relative Bewertung der Eisschutzsystemvarianten

\begin{tabular}{l|l|l|l|l|l}
\hline Konzeptgruppe & Heißluft & Druckluft & Flüssigkeit & $\begin{array}{l}\text { Elektro- } \\
\text { thermisch }\end{array}$ & $\begin{array}{l}\text { Elektro- } \\
\text { mechanisch }\end{array}$ \\
\hline $\begin{array}{l}\text { Verschieben } \\
\text { starrer } \\
\text { Komponenten }\end{array}$ & 0,750 & 0,183 & 0,433 & $\mathbf{0 , 8 5 0}$ & 0,567 \\
\hline $\begin{array}{l}\text { Verformen einer } \\
\text { elastischen } \\
\text { Oberfläche }\end{array}$ & 0,567 & 0,167 & 0,433 & 0,667 & $\mathbf{0 , 5 6 7}$ \\
\hline $\begin{array}{l}\text { Grenzschichtkon- } \\
\text { trolle }\end{array}$ & $\mathbf{0 , 9 5 0}$ & 0,133 & 0,350 & 0,833 & 0,517 \\
\hline
\end{tabular}




\subsubsection{Identifikation idealer Konturen}

Die weitere Bewertung und Gestaltung der Einlasskonzeptgruppen erforderte Kennnisse über ideale und von den Konzepten umsetzbare Reiseflug-Geometrien sowie das daraus resultierende aerodynamische Einsparpotenzial für den Reiseflug bei Mach 0,95, 1,3 und 1,6. Die Bestimmung der Korrelationen zwischen geometrischen und aerodynamischen Parametern sowie geeigneter Geometrien wurde basierend auf einer zweidimensionalen Strömungssimulation mit gekoppelter Antwortflächenoptimierung in Ansys Fluent 18 durchgeführt, vgl. hierzu im Detail die Arbeit von Wöllner [315] sowie die Veröffentlichungen [301], [330], [331], [332].

Dafür erfolgte zuerst die Herleitung eines aerodynamischen Modells durch

- die Identifikation einer Referenzgeometrie,

- die geometrische Parametrisierung des Einlasses,

- die Identifikation aerodynamischer Bewertungskriterien,

- die Modellbildung der Strömungsanalyse und

- die Validierung des Modells mit Hilfe der Fachliteratur.

Daran schloss eine Parameterstudie bestehend aus

- der Definition der Optimierungsaufgabe,

- einer Versuchsplanung (Design of Experiments, DoE),

- der Erstellung von Antwortflächen,

- einer genetischen Optimierung und

- der Überprüfung der ausgewählten Kandidatengeometrien an.

Das Ziel dieser Herangehensweise war es, die für den Reiseflug idealen makroskopischen Dimensionen des Einlasssystems zu identifizieren. Eine detaillierte Variation von Kurvenparametern, vgl. Albert [14], [15], Schnell [16] und Kulfan [333], sollte Bestandteil späterer Entwicklungsphasen sein.

Es ist zu beachten, dass die bestimmten Geometrien durch den Variationsmechanismus des Einlasses realisierbar sein müssen. Zeitgleich müssen Anforderungen bezüglich Masse, Bauraum und Komplexität erfüllt werden. Aus diesen Anforderungen ergeben sich Einschränkungen hinsichtlich geometrischer Parameter, wie der Lippendicke, dem Eintrittsradius oder der Einlasslänge. Daher entsprechen die in der Theorie aerodynamisch idealen Geometrien nicht zwingend den ausgewählten Geometrien für variable Einlässe. 


\section{Referenzgeometrie}

Als Referenzgeometrie wurde eine vereinfachte, achsensymmetrische Geometrie des starren Einlasses der Rolls-Royce Pearl 15-Triebwerksreihe verwendet [334]. Diese Reihe gehört zu den modernsten Triebwerken ziviler Geschäftsreiseflugzeuge, einem potenziellen Hauptanwendungsgebiet variabler Pitot-Einlässe. Anwendung findet die Pearl 15-Triebwerksreihe beispielsweise in der Bombardier Global 6500, die für Reisegeschwindigkeiten von Mach 0,85 und Höchstgeschwindigkeiten bis Mach 0,9 ausgelegt ist [335].

\section{Geometrische Parametrisierung}

Die Referenzgeometrie wurde in Siemens NX12 als parametrisierte Skizze unter Verwendung von quadratischen Bézierkurven und kubischen Polynomzügen erstellt, vgl. Abbildung 4.19. Der Fanradius $r_{2}$ wurde auf $0,64 \mathrm{~m}$ und der maximale Gondelradius $r_{\max }$ auf $0,93 \mathrm{~m}$ festgelegt [316].

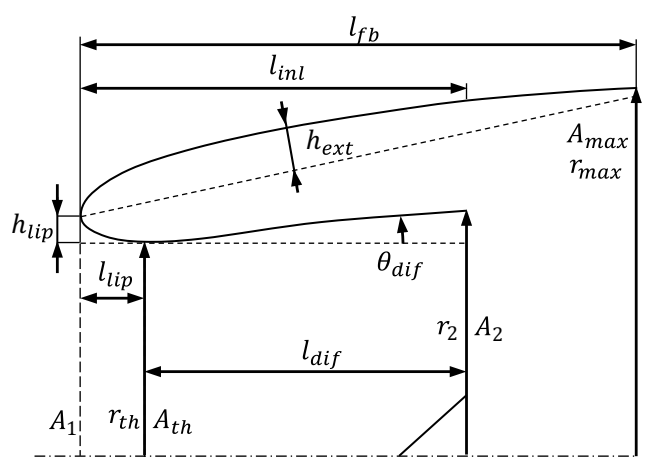

\begin{tabular}{|ll|}
\hline$A_{\text {max }}$ - Maximaler Gondelquerschnitt \\
$A_{1}$ - Einlasseintrittsquerschnitt \\
$A_{t h}$ - Einlasskehlenquerschnitt \\
$A_{2}$ - Fanquerschnitt \\
$h_{\text {lip }}$ - Lippenhöhe \\
$h_{\text {ext }}$ - Gondelvorkörperhöhe \\
$l_{f b}$ - Gondelvorkörperlänge \\
$l_{\text {inl }}$ - Einlasslänge \\
$l_{\text {dif }}$ - Diffusorlänge \\
$l_{\text {lip }}$ - Einlasslippenlänge \\
$r_{\text {max }}$ - Maximaler Gondelradius \\
$r_{\text {th }}$ - Einlasskehlenradius \\
$r_{2}$ - Fanradius \\
$\theta_{\text {dif }}$ - Halber Diffusoröffnungswinkel \\
\hline
\end{tabular}

Abbildung 4.19 Parametrisierung der Einlassgeometrie

Obwohl eine Verkleinerung des maximalen Gondelradius zur Reduzierung des Luftwiderstands genutzt werden könnte, wurde dies nicht untersucht, um den erforderlichen Bauraum innerhalb der Gondel nicht einzuschränken. Dieser ist gegenwärtig für die Integration von Anbauteilen, wie Generatoren, Steuerelektronik und Pumpen, notwendig.

Zur Feststellung der Abhängigkeit der Einlassaerodynamik von der Einlasskontur wurden Variationen der folgenden geometrischen Parameter als Entwurfsvariablen $\boldsymbol{x}_{G}$ in der Optimierung untersucht: 
- der Einlasslänge $l_{i n l}$, bestehend aus Diffusor- $l_{d i f}$ und Lippenlänge $l_{l i p}$,

- der radialen Lippenhöhe $h_{l i p}$ und der Lippenlänge $l_{l i p}$, die zusammen das Lippenellipsenverhältnis $l_{l i p} / h_{l i p}$ ergeben [12],

- des Einlasskehlenradius $r_{t h}$, der zum Lippenkontraktionsverhältnis $\left(r_{t h}+h_{l i p}\right)^{2} /\left(r_{t h}\right)^{2}$ beiträgt [12] und

- der Vorkörperhöhe $h_{e x t}$, die die Wölbung der Außenkontur beschreibt.

\section{Bewertungskriterien}

Die aerodynamischen Kennwerte bezüglich Einlassdruckverhältnis, Strömungsgleichförmigkeit und Luftwiderstand dienten als Bewertungskriterien der Einlassgeometrien, vgl. Abschnitt 2.3.1. Die Erfüllung dieser Kriterien wurde während der Strömungsanalyse mit Hilfe verschiedener Strömungsparameter überwacht.

Das Einlassdruckverhältnis $\pi_{i n l}$ ist aus dem gemittelten Totaldruck in der Fanebene $p_{t 2}$ und unter Freistrombedingungen $p_{t 0}$ gemäß Gleichung (2.1) ermittelbar. Signifikante Einlassdruckverluste und Strömungsablösungen können durch große Machzahlen in der Einlasskehle $M a_{t h}$ hervorgerufen werden. Daher wurde die gemittelte und die maximale lokale Machzahl in der Einlasskehle während der Optimierung überwacht.

Strömungsablösungen tragen zudem zu einer geringeren Strömungsgleichförmigkeit bei. Die Abwesenheit von Strömungsablösungen kann mit Hilfe der dynamischen Viskosität $\mu$, dem Geschwindigkeitsgradient $\partial u / \partial y$ und des Reibungsgesetzes nach Newton

$$
\tau_{W}=\mu\left(\frac{\partial u}{\partial y}\right)_{W}
$$

nachgewiesen werden, indem gezeigt wird, dass die Wandschubspannungen $\tau_{W}$ im Bereich des Einlasses stets positiv sind [336, S. 3], vgl. Abschnitt 2.2.1,

$$
\left(\frac{\partial u}{\partial y}\right)_{W}>0
$$

Der externe Gondelvorkörperwiderstand $D_{\text {nacf,ext }}$ bzw. Überlaufwiderstand, bestehend aus Zulaufwiderstand $D_{\text {pre }}$ und Gondelvorkörperkraft $D_{f b}$, wurde während der Optimierung minimiert. Hierfür war die Bestimmung von Geschwindigkeiten $c$ und Drücken $p$ auf der Fangstromröhre und dem Gondelvorkörper sowie des Triebwerksmassenstroms $\dot{m_{0}}$ erforderlich, vgl. Gleichungen (2.5) und (2.6). 


\section{Modellbildung der Strömungsanalyse}

Die Modellbildung umfasst erforderliche Vereinfachungen, die Bestimmung von Randbedingungen, die automatische Vernetzung des Strömungsraumes, die Auswahl geeigneter Lösungsmethoden und die Ergebnisaufbereitung.

Für das aerodynamische Einsparpotenzial variabler Pitot-Einlässe ist vorrangig die Reiseflugphase relevant, vgl. Abschnitt 2.2.1. In dieser Phase können Anstellwinkel, Seitenwindeinflüsse und verschiedene Schnittgeometrien des Einlasses aufgrund des geringen Einflusses idealisiert vernachlässigt werden. Dies ermöglichte die Verwendung einer zweidimensionalen achsensymmetrischen Analyse, wodurch der Rechenaufwand signifikant verringert wurde. Weiterhin wurde der Rechenaufwand reduziert, indem der Gondelvorkörper isoliert vom verbleibenden Flugzeug untersucht und der Fan nur durch seinen geforderten Massenstrom und Eintrittsdruck modelliert wurde. Dadurch wurden potenzielle Strömungsinteraktionen mit Flugzeugrumpf, Tragflächen und Triebwerksaufhängung vernachlässigt, die in späteren Untersuchungen zu betrachten sind. Für die makroskopische Ermittlung von Geometrietendenzen sind diese Annahmen geeignet und erforderlich.

Zur Untersuchung aller durch die Einlassumströmung auftretenden Effekte im untersuchten Strömungsfeld war dessen Größe entsprechend zu wählen, vgl. Abbildung A.31 in Anhang A.5. Zudem wurden die Randbedingungen des Strömungsfeldes entsprechend der Fanaustrittsbedingungen und der ungestörten Umgebungsgrößen der Atmosphäre festgelegt.

Erforderliche Atmosphärendaten können beispielsweise dem Militärhandbuch MIL-310 [337] entnommen werden. Diese wurden für die Reiseflughöhe des Gulfstream G650ER Geschäftsreiseflugzeuges von $14 \mathrm{~km}$ bestimmt [49]. Für den Druckauslass in der Fanebene wurde eine Machzahl $M a_{2}$ von 0,5 gewählt [8], [11], [47]. Mit Hilfe der idealen Gasgleichung und der Bernoulli-Gleichung wurden die verbleibenden Randbedingungen ermittelt und mit der Fachliteratur abgeglichen [10], [338], [339], vgl. Wöllner [315, S. 45-64] und Tabelle A.5 in Anhang A.5.

Ein strukturiertes C-Netz wurde aufgrund seiner guten Eignung für das Abbilden der Einlassströmung zur Vernetzung des Strömungsfeldes genutzt [340]. Um mögliche Strömungsablösungen detektieren und den Luftwiderstand bestimmen zu können, wurde im wandnahen Bereich stets eine sehr feine Vernetzung für alle Gondelwände automatisiert umgesetzt [341], [342]. Eine feine Vernetzung und somit hohe Grenzschichtauflösung kann durch Werte für den dimensionslosen Wandabstand $y^{+}<1$ erreicht werden [336], [341], vgl. Abbildung A.32. 
Detaillierte Beschreibungen zum dimensionslosen Wandabstand können den Werken von White [343], Schlichting et al. [336] sowie Laurien und Oertel [338] entnommen werden.

Für die Berechnung des Strömungsraumes wurde der Ansatz der Reynoldsgemittelten Navier-Stokes-Gleichungen (RANS, Reynolds-averaged NavierStokes) genutzt [344], [345]. Das RANS-Modell kombiniert akzeptable Genauigkeit und Rechenaufwand. Die Genauigkeit im Bereich der Grenzschicht wurde durch Verwendung des $k$ - $\omega$-SST-Turbulenzmodells erhöht [338], [344]. Dieses erlaubt die Modellierung der turbulenten kinetischen Energie $k$ und der charakteristischen Frequenz $\omega$ von Wirbeln im wandnahen sowie wandfernen Bereich [344], [346], [347]. Dadurch ermöglicht es eine gute Vorhersage des Ablöseverhaltens der Grenzschicht auf glatten Oberflächen [345].

Im Rahmen der Ergebnisaufbereitung wurden die Ergebnisse der Strömungssimulation bezüglich der identifizierten Bewertungskriterien automatisiert analysiert. Auf diesem Weg konnten Einlassdruckverhältnis und Luftwiderstand bestimmt werden, während Fälle mit auftretenden Strömungsablösungen identifiziert und nicht weiterverfolgt wurden.

\section{Validierung des aerodynamischen Modells}

Die Gültigkeit der getroffenen Vereinfachungen und Randbedingungsannahmen wurde durch eine Validierung mit der Fachliteratur und vergleichbaren Studien abgesichert. Dies stellt für diese vorläufige Untersuchung eine kosteneffiziente Alternative zu aufwändigen Windkanaluntersuchungen dar, welche im weiteren Verlauf der Entwicklung durchgeführt werden sollten.

So stimmen die berechneten Randbedingungen [10], [81] und Simulationsergebnisse [36], [348] gute mit der Fachliteratur überein. Beispielsweise präsentiert Robinson [348] die Machzahl-Verteilung im Unterschall für die gesamte Gondel bei einer Fluggeschwindigkeit von Mach 0,82. Dabei zeigt die Machzahl-Verteilung in der Einlassregion eine sehr hohe Übereinstimmung mit den Ergebnissen des zugrunde liegenden Modells bei Mach 0,85 [301], vgl. Abbildung A.33 in Anhang A.5.2. Slater [36] stellt die Ergebnisse einer achsensymmetrischen Simulation scharflippiger Pitot-Einlässe für den Überschallflug bei Mach 1,6 mit einer Fananströmmachzahl von 0,52 dar. Mit der dabei verwendeten scharfen Einlasslippe kann ein anliegender Verdichtungsstoß und somit ein sehr geringer Widerstand realisiert werden [11]. Aufgrund der standzuhaltenden Beanspruchungen, beispielsweise beim Vogelschlag, ist eine scharfe Lippe für den variablen Einlass jedoch nicht zielführend, weshalb nachfolgend ein minimaler Vorderkantendurchmesser von $20 \mathrm{~mm}$ festgelegt wurde. Dadurch ist der senkrechte Verdichtungsstoß von der Vorderkante abgelöst, vgl. Abbildung A.34 
in Anhang A.5.2. Die Machzahlverteilung im verbleibenden Bereich des Einlasses gleicht sich hingegen annähernd mit Slaters Ergebnissen [301].

\section{Antwortflächenoptimierung}

Das Ziel der Optimierung bestand in der Minimierung des Überlaufwiderstands $D_{n a c f, \text { ext }}$ bei verschiedenen Fluggeschwindigkeiten in Abhängigkeit der geometrischen Entwurfsvariablen $\boldsymbol{x}_{G}$ im Entwurfsraum $X$ sowie bei ausreichender Gleichförmigkeit und hohem Einlassdruckverhältnis:

$$
\min _{\boldsymbol{x} \in X} D_{\text {nacf,ext }}\left(\boldsymbol{x}_{G}\right)
$$

Die Entwurfsvariablen $\boldsymbol{x}$ beschreiben Parameter, die während der Optimierung im Entwurfsraum variiert werden [349, S. 239]. Der Entwurfsraum $X$ enthält zudem untere $\boldsymbol{x}^{l}$ und obere $\boldsymbol{x}^{u}$ Schranken für die Entwurfsvariablen:

$$
X=\left\{x \in \mathbb{R}^{7} \mid \boldsymbol{x}^{l} \leq \boldsymbol{x} \leq \boldsymbol{x}^{u}\right\}
$$

Nachfolgend werden geometrische $\boldsymbol{x}_{G}$ und aerodynamische Entwurfsvariablen $\boldsymbol{x}_{A}$ unterschieden:

$$
x=\left[x_{G}^{T}, x_{A}^{T}\right]^{T}
$$

mit

$$
\boldsymbol{x}_{G}=\left[l_{\text {dif }}, l_{\text {lip }} / h_{\text {lip }}, h_{\text {ext }}, h_{\text {lip }}, r_{t h}\right]^{T}
$$

und

$$
\boldsymbol{x}_{A}=\left[M a_{t h, a v g}, \tau_{W}\right]^{T} .
$$

Aus den Anforderungen an variable Einlässe bezüglich Masse, kinematischer Umsetzbarkeit, Bauraum und Komplexität ergeben sich geometrische Begrenzungen, z. B. hinsichtlich Lippendicke und Einlasslänge. Diese sind als Schranken der geometrischen Entwurfsvariablen $\boldsymbol{x}_{G}$ in Tabelle 4.14 aufgelistet.

Zusätzlich wurden Parameterstudien mit verschiedenen Beschränkungswerten für die maximale Diffusorlänge $l_{\text {dif }}$ durchgeführt, um ideale Geometrien für verschiedene kinematische Optionen zu ermitteln, vgl. Abbildung 4.20. 
Tabelle 4.14 Beschränkungen geometrischer Entwurfsvariablen

\begin{tabular}{l|c|c|l|l|l}
\hline \multirow{2}{*}{$\begin{array}{l}\text { Geometrische } \\
\text { Entwurfsvariable }\end{array}$} & \multirow{2}{*}{ Referenzwert } & \multicolumn{2}{|l|}{ Unterschallflug } & \multicolumn{2}{l}{ Überschallflug } \\
\cline { 3 - 6 } & & Min & Max & Min & Max \\
\hline Diffusorlänge $l_{\text {dif }}[\mathrm{mm}]$ & 600 & 600 & 1388 & 750 & 1623 \\
\hline Ellipsenverhältnis $l_{\text {lip }} / h_{\text {lip }}[-]$ & 2,77 & 1,00 & 4,00 & 1,00 & 1,00 \\
\hline Vorkörperhöhe $h_{\text {ext }}[\mathrm{mm}]$ & 98 & 28 & 120 & 28 & 120 \\
\hline Lippenhöhe $h_{\text {lip }}[\mathrm{mm}]$ & 78 & 20 & 80 & 10 & 10 \\
\hline Kehlenradius $r_{\text {th }}[\mathrm{mm}]$ & 586 & 550 & 620 & 550 & 620 \\
\hline
\end{tabular}

Feste Länge

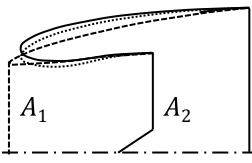

Feste erhöhte Länge

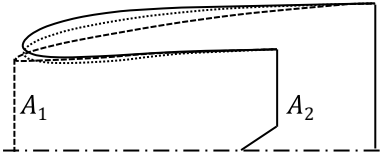

\section{Anpassbare Länge}

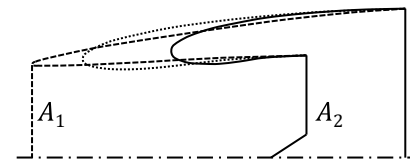

Abbildung 4.20 Optionen der Längenvariation

Die Erfüllung der Anforderungen an die Gleichförmigkeit und das Einlassdruckverhältnis wird durch die aerodynamischen Schranken $\boldsymbol{x}_{A}$ gewährleistet. Dabei werden zum Ausschließen von Strömungsablösungen durchgängig positive Wandschubspannungen $\tau_{W}$ gefordert. Stoßverlusten wird durch mittlere Kehlen-Machzahlen $M a_{t h, a v g}$ von maximal Mach 0,75 vorgebeugt [47].

Zur Erzeugung von Antwortflächen, die eine schnelle Optimierung ermöglichen, wurde die DoE-Methode angewandt. Dabei wurden die Entwurfspunkte mittels optimiertem Latin-Hypercube-Sampling ideal im Entwurfsraum $X$ verteilt. Dadurch sind keine Werte für geometrische Entwurfsvariablen $\boldsymbol{x}_{G}$ identisch, während die Abstände zwischen den Werten maximal sind [350], vgl. Kazula et al. [301]. Anschließend wurden die Strömungsfelder für über 100 Entwurfspunkte automatisiert gelöst. Mit Hilfe einer genetischen Regressionsanalyse wurden aus den gelösten Entwurfspunkten Antwortflächen erzeugt, vgl. Wöllner [315, S. 69]. Die Antwortflächen ermöglichten die Bestimmung der grundlegenden Korrelationen zwischen den geometrischen Parametern und den aerodynamischen Bewertungskriterien.

Weiterhin wurden, basierend auf den Antwortflächen und einer indirekten genetischen Optimierung mit Elite-Strategie, die Kandidatenpunkte für die idealen Geometrien im definierten Entwurfsraum identifiziert, vgl. Kazula et al. [331]. Die 
identifizierten Kandidatenpunkte wurden jeweils simuliert, um die resultierenden Widerstandswerte aus den Antwortflächen zu verifizieren.

\section{Parameterkorrelationen für den Unterschallflug}

Für Fluggeschwindigkeiten von Mach 0,95 wurden bei den gewählten Randbedingungen im untersuchten Parameterbereich zahlreiche Zusammenhänge für die Anteile des externen Gondelvorkörperwiderstands $D_{\text {nacf, ext }}$ ermittelt.

Der Zulaufwiderstand $D_{\text {pre }}$ steigt mit zunehmender Differenz zwischen dem Einlass-Fangquerschnitt $A_{0}$ und dem Eintrittsquerschnitt $A_{1}$ an. Deshalb führen Vergrößerungen des Kehlenradius $r_{t h}$ und des Stirnflächenradius $r_{1}$, zu einem Anstieg des Zulaufwiderstands, vgl. Abbildung 4.21 links oben. Der Stirnflächenradius ergibt sich aus der Summe von Kehlenradius und Lippenhöhe $h_{\text {lip }}$ und entspricht näherungsweise dem Eintrittsradius. Auch eine Vergrößerung der Lippenhöhe resultiert in einem Anstieg des Zulaufwiderstands. Die Länge des Einlasses hat nur einen geringen Einfluss auf den Zulaufwiderstand, vgl. Abbildung 4.21 links unten.
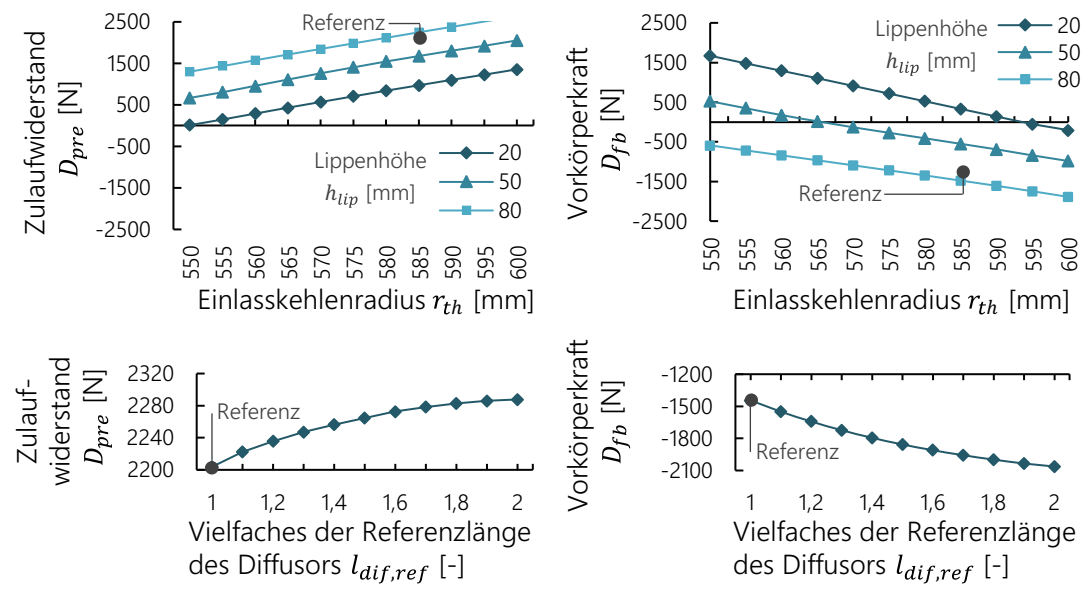

Abbildung 4.21 Abhängigkeit von Zulaufwiderstand und Vorkörperkraft von Lippenhöhe, Kehlenradius und Einlasslänge im Unterschall 
Die Gondelvorkörperkraft $D_{f b}$ nimmt mit zunehmendem Kehlenradius $r_{t h}$ und Stirnflächenradius $r_{1}$ ab, da die Fangstromröhre stärker divergiert, vgl. Abbildung 4.21 rechts unten. Dadurch wird die externe Strömung beschleunigt, wodurch der statische Druck auf der Außenkontur des Einlasses reduziert und eine Saugkraft erzeugt wird, vgl. Abschnitt 2.2.1. Die Referenzgeometrie erzeugt bei Mach 0,95 weiterhin eine große Saugkraft, obwohl sie auch bei langsamen Flugzuständen nachweislich zuverlässig funktioniert. Eine Reduktion der Lippenhöhe $h_{\text {lip }}$ führt bei konstantem Kehlenquerschnitt und Gondeldurchmesser zu einem Anstieg der Vorkörperkraft. Folglich wäre eine scharfe Lippe mit kleinem Kehlenquerschnitt ungeeignet für den Unterschall-Reiseflug.

Eine Verlängerung des Einlasses führt zu einer deutlichen Verringerung der Vorkörperkraft, vgl. Abbildung 4.21 rechts oben. Dies begründet sich durch die veränderte Außenkontur des längeren Vorkörpers [336], vgl. Abbildung 4.22. Durch die längere Kontur wird die gewünschte negative Druckdifferenz auf dem Vorkörper vergleichsweise früh erreicht und anschließend eine fast konstante Geschwindigkeit beibehalten. Dadurch wird die wirkende Saugkraft maximiert. Die kleineren Schwankungen der Druckdifferenz zeigen schwache Verdichtungsstöße (Shocklets) entlang der Kontur an [351, S. 328].

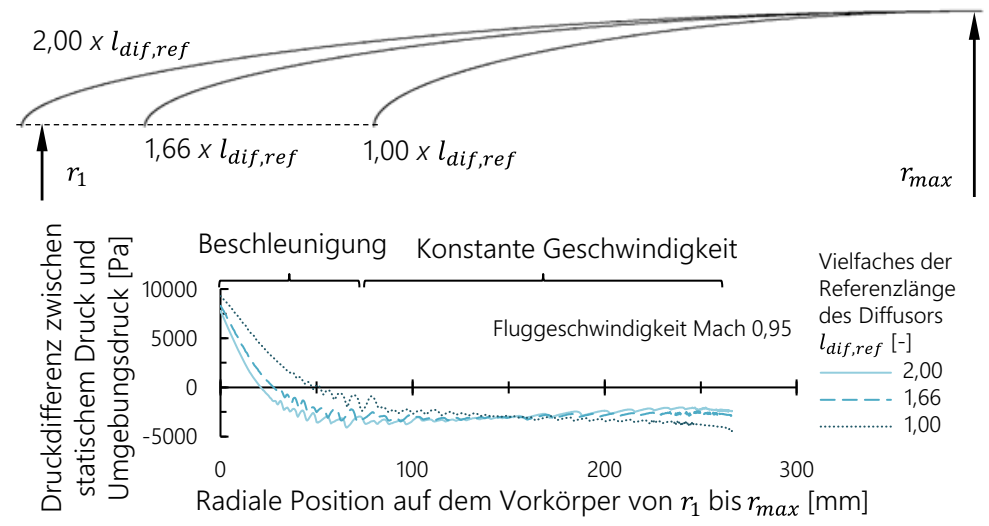

Abbildung 4.22 Druckverteilung auf äußerem Vorkörper bei Mach 0,95 
Der aus der Summe von Zulauf- und Vorkörperwiderstand resultierende Überlaufwiderstand $D_{\text {nacf,ext }}$ kann durch die Erhöhung der Einlasslänge signifikant verringert werden, vgl. Abbildung 4.23 rechts oben. Ein Mindestmaß der Konturwölbung $h_{\text {ext }}$ ist erforderlich, um eine ausreichende Beschleunigung der Strömung zu Beginn der äußeren Kontur zu ermöglichen, vgl. Abbildung 4.23 unten. Somit hat eine Verringerung der Konturwölbung keinen positiven Einfluss. Eine Verringerung der Lippenhöhe $h_{\text {lip }}$ resultiert in einem größeren Überlaufwiderstand. Der Einfluss des Kehlenradius $r_{t h}$ ist für verhältnismäßig dicke Einlasslippen gering, vgl. Abbildung 4.23 links oben.
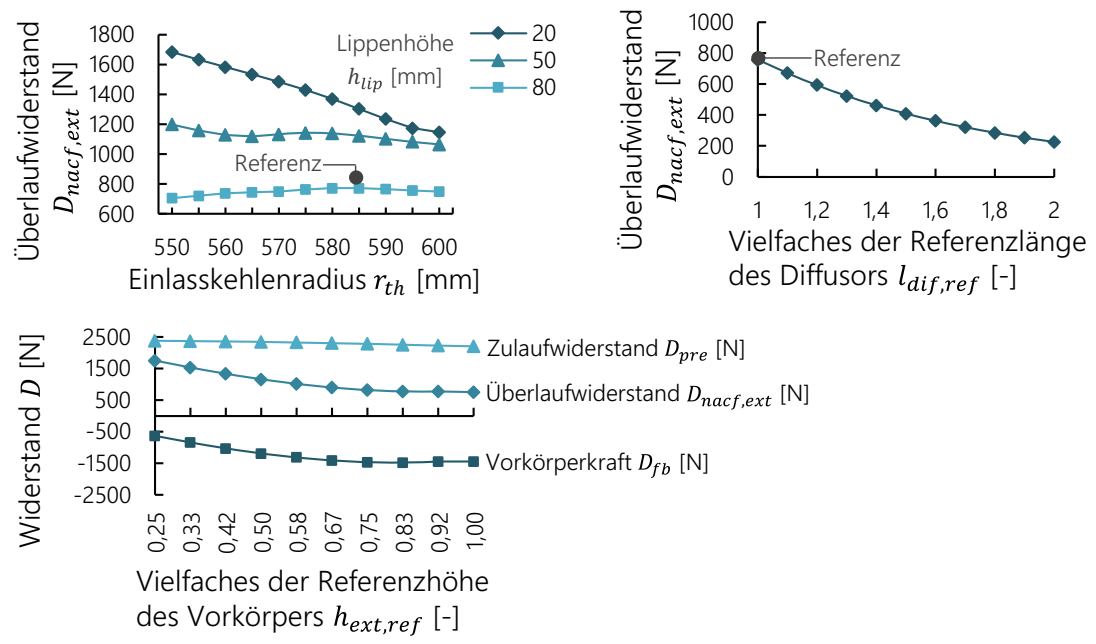

Abbildung 4.23 Abhängigkeit des Einlasswiderstands von geometrischen Parametern

Das Einlassdruckverhältnis ist primär vom Kehlenradius $r_{t h}$ abhängig. Kleinere Kehlenradien führen zu größeren Machzahlen in der Einlasskehle $M a_{t h}$. Dadurch können lokale Verdichtungsstöße und Grenzschichtaufdickungen auftreten, die sich negativ auf das Einlassdruckverhältnis auswirken.

Für die gegebenen Randbedingungen resultiert ein Kehlenradius $r_{t h}$ von $560 \mathrm{~mm}$ in einer durchschnittlichen Kehlen-Machzahl von 0,7 und einem sehr guten Einlassdruckverhältnis von 0,995, vgl. Abbildung 4.24. Bei einem Radius von $550 \mathrm{~mm}$ ergibt sich ein um 0,3 Prozent verringertes Einlassdruckverhältnis. Hieraus würde bei einem Triebwerksschub von $20 \mathrm{kN}$ ein umgerechneter Schubverlust von $100 \mathrm{~N}$ resultieren [11, S. 11], vgl. Abschnitt 2.3.1. Für kleiner werdende Kehlenradien nimmt dieser Verlust stark zu. 
Abbildung 4.24

Abhängigkeit des

Einlassdruckverhältnis vom

Kehlenradius

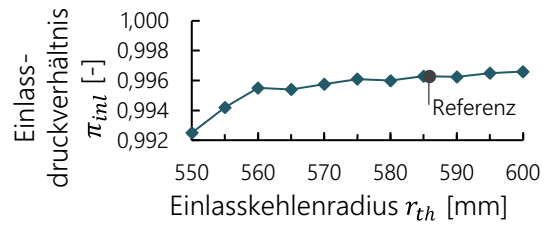

Die radiale Gleichförmigkeit der Fananströmung kann im Reiseflug vorrangig durch Strömungsablösungen aufgrund von Verdichtungsstößen im Bereich der Einlasslippe und aufgrund ungeeigneter Diffusoröffnungswinkel beeinträchtigt werden. Verdichtungsstöße können bei lokalen Machzahlen, die größer als 1 sind, auftreten. Bei einer Konfiguration mit einem Kehlenradius $r_{t h}$ von $550 \mathrm{~mm}$ und einer Lippenhöhe $h_{\text {lip }}$ von $50 \mathrm{~mm}$ ergeben sich für Ellipsenverhältnisse $l_{\text {lip }} / h_{\text {lip }}$, die kleiner als 2,7 sind, lokale Machzahlen größer 1, vgl. Abbildung 4.25 links. Gleichzeitig fällt in diesen Fällen die minimale axiale Wandschubspannung zwischen Einlasseintritt und Kehle in negative Bereiche, was lokale Rückströmungen und Strömungsablösungen aufzeigt.

Die Werte für den halben Diffusoröffnungswinkel $\theta_{\text {dif }}$, vgl. Abbildung 2.9, überschreiten im untersuchten Parameterbereich nicht die empfohlenen $8^{\circ}$, wodurch keine negativen Wandschubspannungen auftreten. Folglich entstehen keine Strömungsablösungen aufgrund ungeeigneter Diffusoröffnungswinkel, vgl. Abbildung 4.25 rechts.
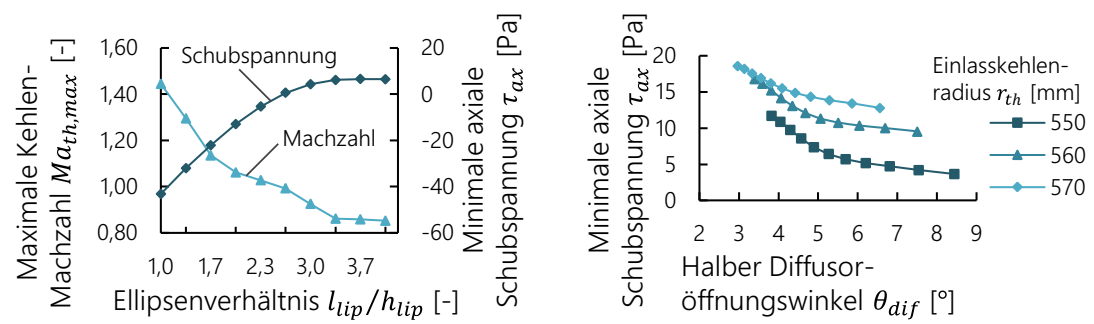

Abbildung 4.25 Einfluss geometrischer Einlassparameter auf die axiale Wandschubspannung

\section{Parameterkorrelationen für den Überschallflug}

Im Überschallbetrieb steigt der Zulaufwiderstand $D_{\text {pre }}$, wie auch im Unterschall, mit zunehmendem Kehlenradius $r_{t h}$ an, vgl. Abbildung 4.26 links oben. Jedoch ist der Widerstandsanstieg hier deutlich größer als im Unterschallbereich. Zwischen Einlasslänge und Zulaufwiderstand konnte kein eindeutiger Zusammenhang bestimmt werden. 
Die Gondelvorkörperkraft $D_{f b}$ hängt noch stärker von der Fluggeschwindigkeit ab als der Zulaufwiderstand. So führt eine Erhöhung der Fluggeschwindigkeit von Mach 1,3 auf Mach 1,6 zu einer Verdopplung der Vorkörperkraft, vgl. Abbildung 4.26 unten. Eine Vergrößerung des Kehlenradius $r_{t h}$ führt in Kombination mit dem konstanten Gondelradius $r_{\max }$ zu einer deutlichen Verringerung der Vorkörperkraft. Dies begründet sich mit einer Reduzierung der projizierten Querschnittsfläche sowie einer geringeren Strömungsumlenkung entlang des Vorkörpers. Die geringere Strömungsumlenkung resultiert in einer verringerten Anzahl Verdichtungsstöße, die zudem abgeschwächt sind.

Die Verringerung der relativen Strömungsumlenkung entlang des Vorkörpers kann auch durch seine Verlängerung erfolgen, wodurch die Vorkörperkraft am besten reduziert werden kann. Der Effekt der Widerstandsverringerung nimmt dabei mit zunehmender Länge des Vorkörpers ab, vgl. Abbildung 4.26 oben rechts. Durch die Verlängerung der Kontur erfolgt die stärkste Umlenkung und Beschleunigung der Strömung im Bereich der Vorderkante, vgl. Abbildung 4.27. In Kombination mit einer geringen Wölbung werden somit stromabwärts die auftretenden Verdichtungsstöße abgeschwächt. Dadurch wird die Druckdifferenz zwischen dem statischen Druck auf dem Vorkörper und dem Umgebungsdruck reduziert. Aufgrund des senkrechten Verdichtungsstoßes vor dem Einlass ist der
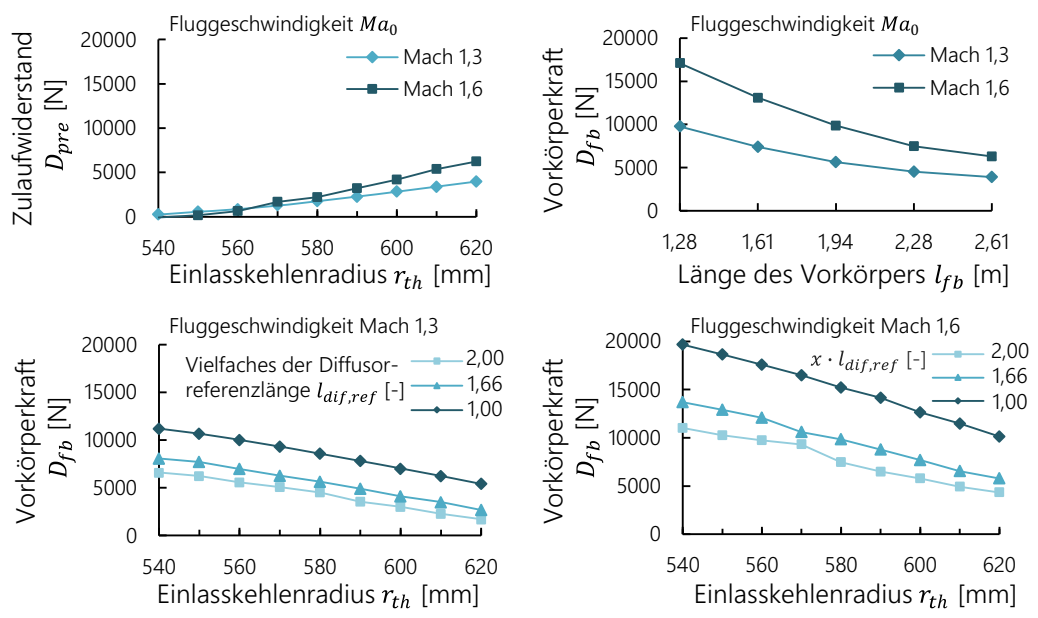

Abbildung 4.26 Abhängigkeit von Zulaufwiderstand und Vorkörperkraft von Lippenhöhe, Kehlenradius und Einlasslänge im Überschall 
statische Druck jedoch erhöht, weshalb er für den Großteil des Vorkörpers den Umgebungsdruck überschreitet und das Erzeugen einer Saugkraft verhindert.

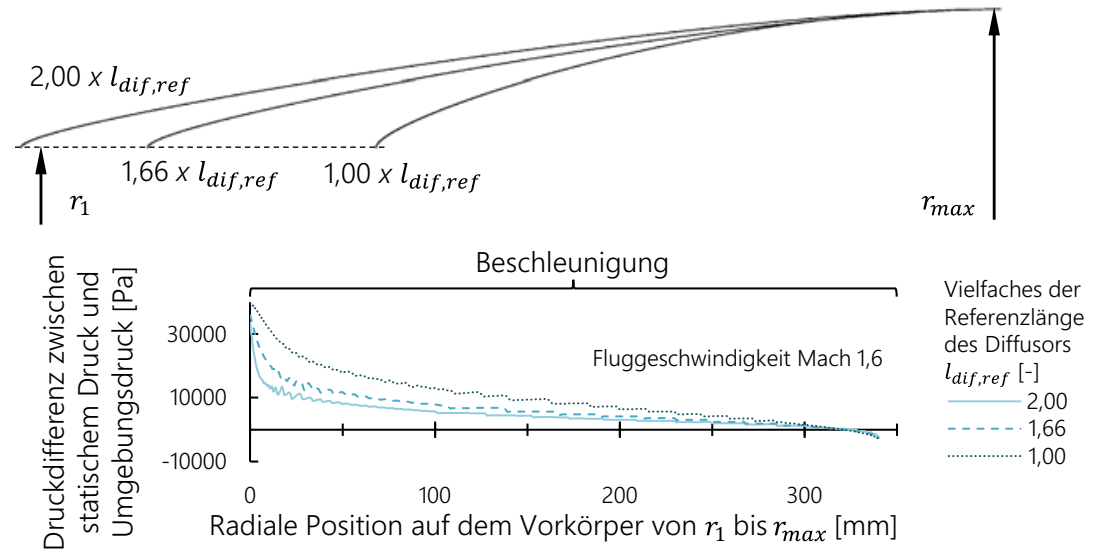

Abbildung 4.27 Druckverteilung auf äußerem Vorkörper bei Mach 1,6

Durch eine Minimierung der Lippenhöhe $h_{\text {lip }}$ könnte die projizierte Einlassstirnfläche verringert und somit die Vorkörperkraft reduziert werden. Von einer Reduktion der Lippenhöhe $h_{\text {lip }}$ unter Werte von $20 \mathrm{~mm}$ wurde jedoch aufgrund der strukturell erforderlichen Mindesthöhe abgesehen.

Der Überlaufwiderstand $D_{\text {nacf, ext }}$ kann primär durch einen längeren Vorkörper mit daraus folgender geringerer Wölbung und flacherem Anströmwinkel der Außenkontur reduziert werden, vgl. Abbildung 4.28. Jedoch ist eine gewisse Wölbung erforderlich, um starke Verdichtungsstöße beim Übergang zum maximalen Gondelradius $r_{\max } \mathrm{zu}$ vermeiden. Darüber hinaus verringert sich bei längeren Einlässen der Einfluss einer Variation des Kehlenradius $r_{t h}$.

Aufgrund des im Überschallbetrieb entstehenden senkrechten Verdichtungsstoßes vor dem Pitot-Einlass, können nur theoretische Einlassdruckverhältnisse $\pi_{i n l}$ von etwa 0,98 bei einer Fluggeschwindigkeit von Mach 1,3 bzw. 0,90 bei Mach 1,6 erreicht werden, vgl. Abschnitt 2.3.1. Bei den untersuchten Fällen, bei denen keine Strömungsablösungen auftreten, wurden sehr gute Werte für das Einlassdruckverhältnis von 0,977 bei Mach 1,3 bzw. 0,893 bei Mach 1,6 bestimmt, was mit den Werten der Studie von Slater [36] übereinstimmt, vgl. Wöllner [315, S. 90]. 


\section{Abbildung 4.28}

Abhängigkeit des

Einlasswiderstands vom

Kehlenradius

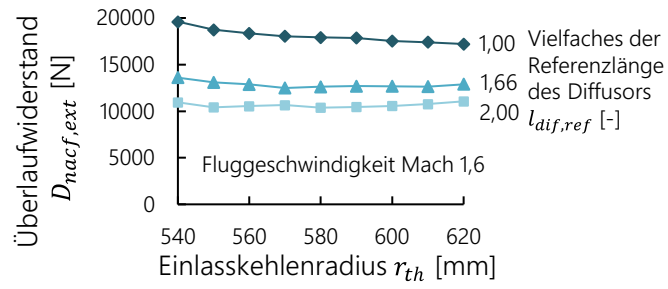

Die Strömungsgleichförmigkeit wird im Überschallbetrieb vorrangig durch Grenzschichtablösungen beeinträchtigt. Diese können bei ungeeigneten Kehlenund Eintrittsradien auftreten. Bei zu kleinen Eintrittsradien steigt die Machzahl in der Kehle stark an, wodurch lokale Verdichtungsstöße auf der Einlasslippe auftreten und zu Strömungsablösungen führen können, vgl. Abbildung A.35 in Anhang A.5. Ist der Eintrittsradius zu groß gewählt, divergiert die Fangstromröhre stärker, was zu einer erhöhten Umlenkung und Beschleunigung der Strömung entlang der Vorderkante führt. Dadurch können auf der Außenseite des Vorkörpers Verdichtungsstöße mit zugehörigen Verlusten und Strömungsablösungen auftreten, vgl. Abbildung A.36 in Anhang A.5. Bei den gewählten Bedingungen traten für Kehlenradien $r_{t h}$ im Intervall von 580 bis $620 \mathrm{~mm}$ keine Strömungsablösungen auf. Jedoch sind diese Werte stark von den getroffenen Annahmen abhängig, beispielsweise bezüglich des Triebwerksmassenstroms.

\section{Geometrieauswahl}

Zur Minimierung der Komplexität der variablen Pitot-Einlässe bis Mach 1,6 wurde im Rahmen dieser ersten Konzeptstudie die minimale Anzahl umzusetzender Geometrien ausgewählt, die einen zuverlässigen und effizienten Betrieb ermöglicht. Dabei ist das geringe Potenzial variabler Pitot-Einlässe im reinen Unterschallbetrieb zugleich ein Vorteil, da eine einzige Geometrie sowohl bei langsamen Flugbedingungen als auch im Unterschallreiseflug zuverlässig und relativ effizient eingesetzt werden kann. Für den Überschallbetrieb wurde ebenfalls eine einzige Geometrie ausgewählt, die bei einer Fluggeschwindigkeit von Mach 1,6 am besten geeignet ist.

Somit muss ein möglicher Stellmechanismus nur zwischen zwei Konturen wechseln können. Dies betrifft insbesondere die Konzeptgruppen des Verschiebens starrer Komponenten und der elastischen Oberflächenverformung. Für Konzepte mit aerodynamischer Grenzschichtbeeinflussung wird die Überschallkontur starr implementiert, während im Unterschallbetrieb Strömungsablösungen verhindert werden. 
Weiterhin kann die Komplexität aller Konzepte durch den Verzicht auf die Variation der Länge und des Vorderkantendurchmessers signifikant verringert werden. Folglich wurden entsprechend der identifizierten Parameterkorrelationen und der Optimierungsergebnisse Tendenzen idealer Geometrien ermittelt und Geometrien gewählt [301], vgl. Tabelle 4.15 und Abbildung 4.29.

Tabelle 4.15 Tendenzen idealer und gewählter Geometrien

\begin{tabular}{l|l|l|l|l|l}
\hline \multirow{2}{*}{ Geometrischer Parameter } & \multirow{2}{*}{ Referenz } & \multicolumn{2}{l|}{ Unterschallflug } & \multicolumn{2}{l}{ Überschallflug } \\
\cline { 3 - 6 } & & Ideal & Gewählt & Ideal & Gewählt \\
\hline Diffusorlänge $l_{\text {dif }}[\mathrm{mm}]$ & 600 & Maximal & 1388 & Maximal & 1623 \\
\hline Ellipsenverhältnis $l_{\text {lip }} / h_{\text {lip }}[-]$ & 2,7 & $>3$ & 3,5 & 1,0 & 1,0 \\
\hline Vorkörperhöhe $h_{\text {ext }}[\mathrm{mm}]$ & 98 & Hoch & 112 & Niedrig & 56 \\
\hline Lippenhöhe $h_{\text {lip }}[\mathrm{mm}]$ & 78 & Hoch & 70 & Niedrig & 10 \\
\hline Kehlenradius $r_{t h}[\mathrm{~mm}]$ & 586 & $\geq 560$ & 560 & 580 bis 620 & 620 \\
\hline
\end{tabular}

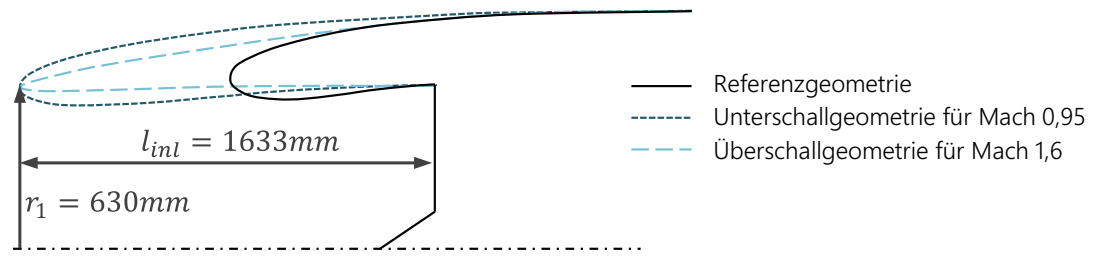

Abbildung 4.29 Gewählte Geometrien variabler Einlässe

Obwohl die Unterschallgeometrie nicht bei langsamen Flugbedingungen simuliert und getestet wurde, sollte sie zuverlässig bei diesen Bedingungen ihre Funktion erfüllen. Dies ist in nachfolgenden Studien zu zeigen. Gründe für die angenommene Funktionalität bestehen in den konservativ gewählten Geometrieparameterwerten, insbesondere dem erhöhten Ellipsenverhältnis und der größeren Länge, wodurch die Strömungsumlenkung auf einer längeren Strecke erfolgt. Zudem ist der Diffusoröffnungswinkel kleiner als $8^{\circ}$ und die durchschnittliche Kehlen-Machzahl unterschreitet stets Mach 0,7. 


\section{Geometrieverifizierung}

Da die Geometrien mit Hilfe der Antwortflächen bestimmt wurden, erfolgte eine jeweilige Simulation dieser zur Sicherstellung der Erfüllung der aerodynamischen Bewertungskriterien. Diese Simulationen bei Fluggeschwindigkeiten von Mach 0,95 und 1,6 zeigen, dass die bestimmten Geometrien deutlich besser für die jeweiligen Fluggeschwindigkeiten geeignet sind als die Referenzkontur, die für Mach 0,85 ausgelegt ist.

So sind die auftretenden Überschallgebiete und die maximalen lokalen Geschwindigkeiten für die gewählte Unterschallgeometrie bei Mach 0,95 deutlich kleiner als die der Referenz, vgl. Abbildung 4.30 oben. Dies resultiert in einer Verringerung des Überlaufwiderstands $\Delta D_{\text {nacf,ext }}$ von über $700 \mathrm{~N}$ bzw. $97 \%$, vgl. Tabelle 4.16. Im Überschallbetrieb reicht der senkrechte Verdichtungsstoß bei der gewählten Überschallgeometrie deutlich näher an die Einlasskontur heran als bei der Referenzgeometrie und ist zudem abgeschwächt, vgl. Abbildung 4.30 unten. Dadurch wird der Überlaufwiderstand $D_{\text {nacf, ext }}$ des Einlasses um über $9.000 \mathrm{~N}$ bzw. über $40 \%$ reduziert.

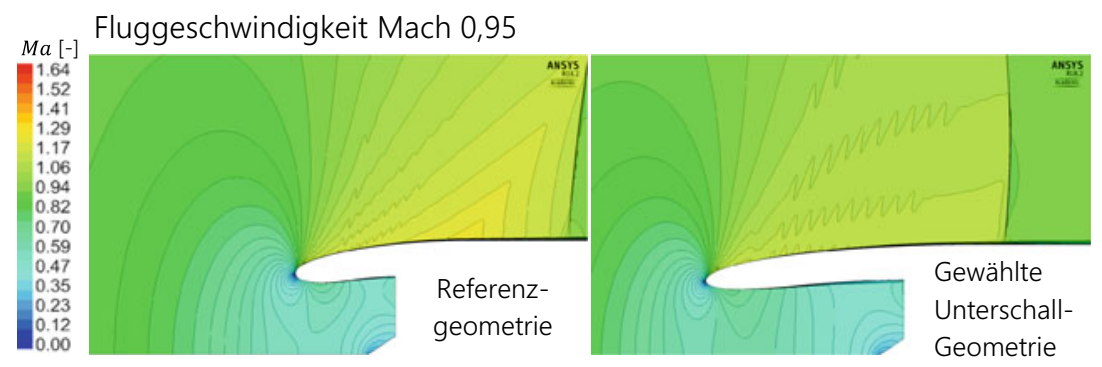

Fluggeschwindigkeit Mach 1,6

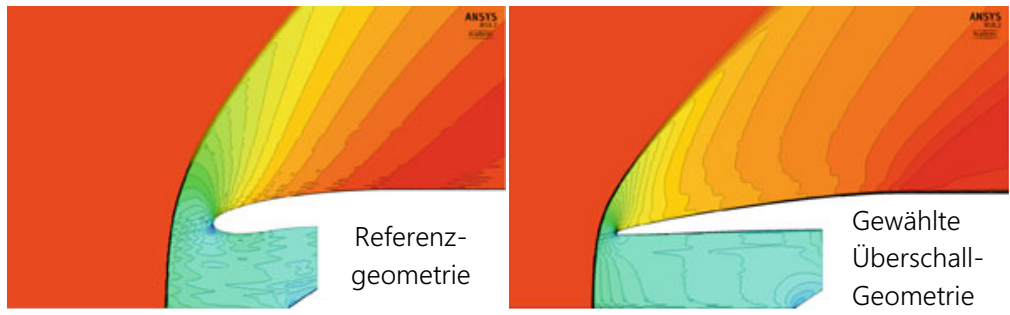

Abbildung 4.30 Machzahlverteilungen der Referenz und der ausgewählten Geometrien 
Tabelle 4.16 Vergleich der Referenzkontur mit den ausgewählten Konturen

\begin{tabular}{l|c|l|l|l}
\hline \multirow{2}{*}{ Bewertungskriterium } & \multicolumn{2}{l|}{$\begin{array}{l}\text { Unterschallflug bei } \\
\text { Mach 0,95 }\end{array}$} & \multicolumn{2}{l}{$\begin{array}{l}\text { Überschallflug bei } \\
\text { Mach 1,6 }\end{array}$} \\
\cline { 2 - 5 } & $\begin{array}{l}\text { Referenz- } \\
\text { kontur }\end{array}$ & $\begin{array}{l}\text { Unterschall- } \\
\text { kontur }\end{array}$ & $\begin{array}{l}\text { Referenz- } \\
\text { kontur }\end{array}$ & $\begin{array}{l}\text { Überschall- } \\
\text { kontur }\end{array}$ \\
\hline Zulaufwiderstand $D_{\text {pre }}[\mathrm{N}]$ & 2180 & 1360 & 8800 & 4100 \\
\hline Vorkörperkraft $D_{f b}[\mathrm{~N}]$ & -1440 & -1340 & 10900 & 6520 \\
\hline Überlaufwiderstand $D_{\text {nacf,ext }}[\mathrm{N}]$ & 740 & 20 & 19700 & 10620 \\
\hline $\begin{array}{l}\text { Widerstandsreduktion } \Delta D_{\text {nacf, ext }} \\
{[\mathrm{N}]}\end{array}$ & - & 720 & - & 9080 \\
\hline Einlassdruckverhältnis $\pi_{\text {inl }}[-]$ & 0,996 & 0,996 & 0,892 & 0,893 \\
\hline
\end{tabular}

$\mathrm{Zu}$ beachten ist bei diesen Ergebnissen, dass ein starrer Überschall-PitotEinlass voraussichtlich mit einer dünneren Geometrie ausgelegt werden würde, wodurch sich die identifizierten Ersparnisse verringern würden. Allerdings wäre in diesem Fall der Vorteil des größeren Betriebsbereichs variabler Einlässe stärker ausgeprägt, beispielsweise bei Seitenwind.

Aus Simulationen der Unterschallkontur bei Überschallbedingungen geht ein Überlaufwiderstand $D_{\text {nacf, ext }}$ von $15660 \mathrm{~N}$ hervor [315, S. 95]. Für den umgekehrten Fall beträgt der Überlaufwiderstand $D_{\text {nacf, ext }} 800$ N [315, S. 95]. Diese deutlichen Anstiege des Widerstands, die teilweise über das Niveau der Referenzkontur hinausreichen, offenbaren die Notwendigkeit variabler Einlässe für einen effizienten Betrieb sowohl im Unterschall- als auch im Überschall-Reiseflug.

\section{Aerodynamisches Einsparpotenzial}

Die Reduzierung des Luftwiderstands durch Verwendung variabler Einlässe ermöglicht eine größere Flugreichweite $R$, einen verringerten Kraftstoffverbrauch $\dot{m}_{f u e l}$ oder eine geringere mitzuführende Kraftstoffmenge $m_{f u e l}$ bzw. eine erhöhte Nutzlast. Diese Vorteile sind mit Hilfe der Breguet'schen Reichweitenformel [10, S. 454] näherungsweise quantifizierbar, vgl. Anhang A.5.3.

Der Widerstandskoeffizient $c_{D}$ eines Flugzeugs, das Triebwerke mit variablen Einlässen nutzt, hat einen großen Einfluss auf die möglichen Verbesserungen bezüglich Flugreichweite $R$ und Kraftstoffverbrauch $\dot{m}_{f u e l}$. Während die meisten Unterschall-Geschäftsreiseflugzeuge zwei Triebwerke nutzen, sind bei einigen Überschall-Konzepten, wie der Aerion AS2, drei Triebwerke vorgesehen [45]. Deshalb werden im Folgenden Konfigurationen mit zwei und drei Triebwerken vorgestellt. Der Einsatz variabler Einlässe im Unterschall bei Mach 0,95 
in einem Flugzeug mit zwei Triebwerken und mit einem üblichen $c_{D}$-Wert von 0,025 ermöglicht einen Reichweitengewinn von etwa $5 \%$, während im Überschall bei Mach 1,6 mit drei Triebwerken und einem $c_{D}$-Wert von $0,035 \mathrm{ca} .35 \%$ Reichweitengewinn möglich sind [352], [353], [354], [355], vgl. Abbildung 4.31.
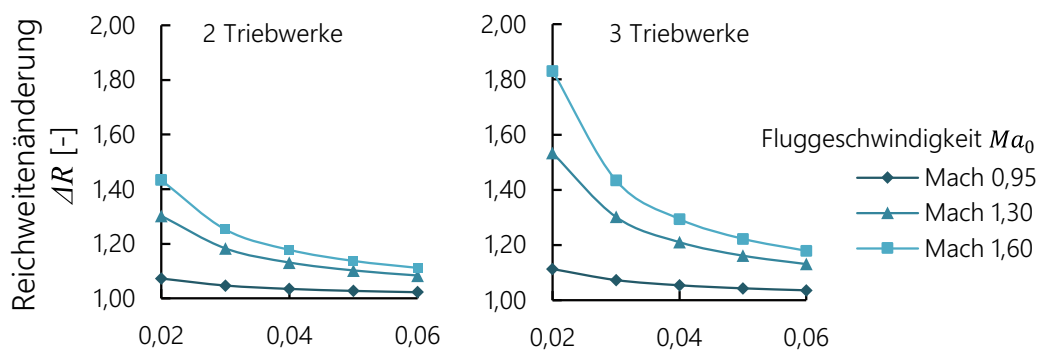

Widerstandsbeiwert $c_{D}[-] \quad$ Widerstandsbeiwert $c_{D}[-]$

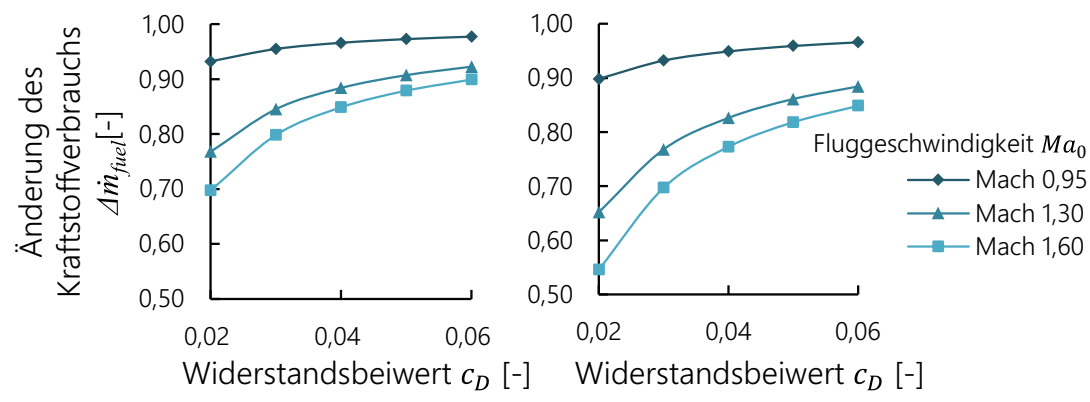

Abbildung 4.31 Änderung von Reichweite und Kraftstoffverbrauch durch Verwendung variabler Einlässe bei verschiedenen Maximalgeschwindigkeiten und Widerstandsbeiwerten

Die Masse variabler Einlässe ist durch die längere Geometrie und die erforderlichen Variationsmechanismen erhöht. Der Einfluss dieser zusätzlichen Masse kann ebenfalls mittels der Breguet'schen Reichweitenformel bestimmt werden. Hierfür wurde eine maximal zulässige Startmasse $m_{t o, \max }$ von $47.000 \mathrm{~kg}$ gewählt, die etwa $1.800 \mathrm{~kg}$ mehr beträgt als die der Gulfstream G650ER oder der Bombardier Global 6500 [49], [335]. Zudem wurde für Fluggeschwindigkeiten von Mach 0,85 und Mach 0,95 ein Widerstandsbeiwert $c_{D}$ von 0,025 und für Mach 1,3 bzw. Mach 1,6 ein $c_{D}$-Wert von 0,035 gewählt.

Unterschallflugzeuge bis Mach 0,95 mit zwei Triebwerken erreichen bei Verwendung variabler Einlässe mit einer konservativen Zusatzmasse von $500 \mathrm{~kg}$ 
je Einlass keinen merklichen Reichweitengewinn, vgl. Abbildung 4.32. Überschallflugzeuge bis Mach 1,6 mit drei Triebwerken ermöglichen bei der gleichen Zusatzmasse pro Einlass eine um $20 \%$ erhöhte Reichweite.
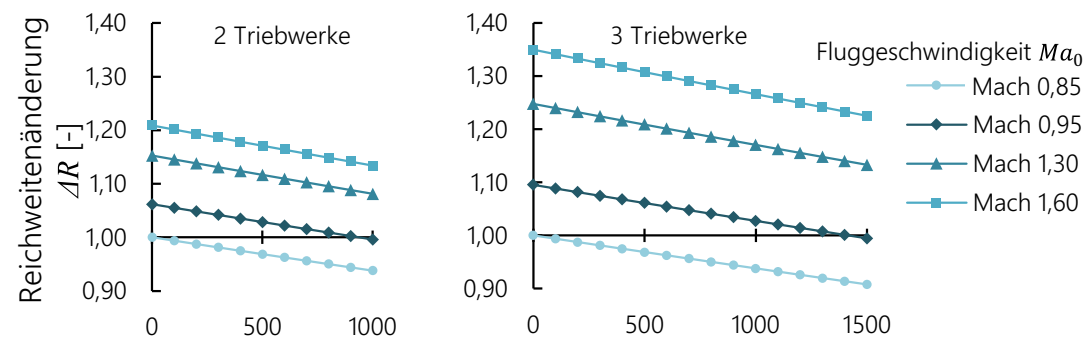

Zusätzliche Gesamtmasse $\Delta \mathrm{m}[\mathrm{kg}] \quad$ Zusätzliche Gesamtmasse $\Delta \mathrm{m}[\mathrm{kg}]$

Abbildung 4.32 Abhängigkeit der Reichweitenänderung von der Zusatzmasse variabler Einlässe

\section{Vorteile und Limitierungen der Herangehensweise}

Der verwendete Ansatz ermöglicht die zeit- und kosteneffiziente Bestimmung von Korrelationen zwischen geometrischen und aerodynamischen Einlassparametern sowie die Ermittlung idealer Einlassgeometrien für Unter- und ÜberschallReiseflugbedingungen. Aus den Vereinfachungen bei der Modellbildung, den getroffenen Annahmen für die Randbedingungen und der Antwortflächenoptimierung ergeben sich Einschränkungen bezüglich der Ergebnisgenauigkeit, die für eine erste Konzeptstudie tolerierbar sind [301].

Es wurden ausschließlich Reiseflugbedingungen eingehend untersucht. Untersuchungen bezüglich verbleibender Flugphasen, z. B. Start, Steigflug, Seitenwind und Windmilling, könnten ebenfalls zu verbesserten Geometrien für diese Bedingungen führen. Dies könnte eine höhere Widerstandsfähigkeit gegen Seitenwind, höhere Steigraten und verringerten Lärm in Flughafennähe ermöglichen. Da diese Betriebsbedingungen jedoch deutlich komplexer als der Reiseflug sind, können die zugehörigen Untersuchungen nur bei einer anschließenden experimentellen Validierung durch Versuche wertvolle Ergebnisse bereitstellen. Während für nachfolgende Entwicklungsphasen folglich zusätzliche Analysen und Versuche empfohlen werden, können die Ergebnisse des vorgestellten Ansatzes als Anhaltspunkte für den weiteren Verlauf der vorliegenden Konzeptstudie herangezogen werden. 


\subsubsection{Konzeptgruppenbewertung}

Die abschließende Auswahl einer Konzeptgruppe erfolgte, wie die Auswahl geeigneter Eisschutzsysteme, mit Hilfe einer gewichteten Punktbewertung. Dafür wurde die Erfüllung der Bewertungskriterien aus Abschnitt 4.1.4 durch die jeweilige Konzeptgruppe basierend auf dem Bewertungssystem aus Abschnitt 4.4.2 durch eine Gruppe von vier Ingenieuren untersucht.

Die Konzeptgruppen für variable Einlässe, die die Geometrie durch Grenzschichtbeeinflussung oder durch Verformen einer elastischen Oberfläche variieren, gewährleisten eine sehr gute aerodynamische Funktionalität. Beide Konzeptgruppen ermöglichen bei einer Geometrieanpassung im geforderten Bereich eine glatte, lücken- und stufenlose Strömungskontur. Die Konzeptgruppe des Verschiebens starrer Komponenten weist mögliche Einschränkungen hinsichtlich der aerodynamischen Funktionalität auf. Diese Einschränkungen ergeben sich aus den kleinen Stufen und Lücken zwischen den Segmenten, der nicht ideal kreisförmigen Querschnittsgeometrie und den Einschränkungen bei der Anpassung des Eintrittsdurchmessers.

Die Integration dieser Konzeptgruppe erfordert ein Stellsystem, eine Erweiterung des vor Vereisung zu schützenden Bereichs und eine erhöhte Teileanzahl. Dennoch ist diese Konzeptgruppe im Vergleich zu den beiden verbleibenden Optionen wesentlich einfacher in den Einlass zu integrieren. Die Konzeptgruppe der elastischen Oberflächenverformung erfordert eine komplexe interne Stützstruktur, innovative Lösungen für Eisschutz und Lärmminderung sowie ein Stellsystem. Darüber hinaus ist der Einfluss der Umgebungsbedingungen auf das Oberflächenmaterial zu untersuchen. Die Konzepte der Grenzschichtbeeinflussung erfordern Pumpen, Rohre und zahlreiche ansteuerbare Schlitze, die vor Eis und Schmutz geschützt werden müssen.

Die erforderlichen Komponenten für die Grenzschichtbeeinflussung resultieren in einer erhöhten Masse, diese kann jedoch teilweise durch die schlanke, starre Geometrie ausgeglichen werden. Die Konzeptgruppen der starren Komponentenverschiebung und der elastischen Oberflächenverformung erfordern hingegen Variationsmechanismen, einschließlich einer Aktorik, die zu einer Massenzunahme führt. Beide Konzeptgruppen sind zudem segmentiert, weshalb ihre strukturelle Dimensionierung gegen Beanspruchungen, wie Vogelschläge, eine erhöhte Masse nach sich zieht. Weiterhin benötigt die Konzeptgruppe der elastischen Oberflächenverformung für Belastungsaufnahme und für das Beibehalten der eingestellten Geometrie eine Stützstruktur mit entsprechender zusätzlicher Masse. 
Die Sicherheit von Konzepten der Gruppe verschiebbarer starrer Komponenten kann durch Vorkehrungen gewährleistet werden. So kann Vogelschlägen durch eine entsprechende Dimensionierung widerstanden werden. Für den Fall einer Aktorfehlfunktion kann automatisch eine versagenssichere Geometrie eingestellt und durch einen unabhängigen Feststellmechanismus gesichert werden. Ein mögliches zusätzliches Feuerrisiko durch ungeeignete Kombinationen von Stellsystem und Eisschutzsystem kann mittels konstruktiver Vorkehrungen beherrscht werden. Für die Konzeptgruppe elastischer Oberflächenverformung gelten für Aktorversagen und das Eindämmen des Feuerrisikos die gleichen Möglichkeiten. Jedoch sind diese Konzepte anfälliger für gefährliches Materialversagen durch Vogeloder Fremdkörpereinschläge. Die Konzeptgruppe der Grenzschichtbeeinflussung verwendet eine schlanke, starre Geometrie, die anfällig für Strömungsablösungen beim Start und im Steigflug sein kann. Für den Fall einer Fehlfunktion des Grenzschichtbeeinflussungssystems können gefährliche Ereignisse eintreten. Diese könnten auf Kosten des aerodynamischen Einsparpotenzials durch eine ablösungsresistentere starre Ausgangsgeometrie vermieden werden.

Nach Berücksichtigung der erhöhten Teilezahl, der flexiblen Leitungen, des Leistungs- und Wartungsbedarfs des Stellsystems und der Reibung zwischen Segmenten, bieten Konzepte der Verschiebung starrer Komponenten weiterhin gute Lebenswegkosten und Zuverlässigkeit. Konzepte mit elastisch verformbaren Oberflächenmaterialien haben aufgrund von Erosion und anderen Umwelteinflüssen eine stark begrenzte Lebensdauer, was in erhöhtem Wartungsaufwand resultiert. Abgesehen von der großen Schlitzanzahl und dem damit einhergehenden Bedarf an Schutzvorkehrungen und Wartung verbinden grenzschichtbeeinflussende Konzepte hohe Zuverlässigkeit mit niedrigen Betriebskosten.

Wenngleich alle Konzeptgruppen einen erhöhten Herstellungsaufwand erfordern, wird nur dem verformbaren Oberflächenmaterial der zugehörigen Konzeptgruppe eine signifikante Erhöhung der Herstellungskosten zugeschrieben. Zudem erfordert diese Gruppe und die der Grenzschichtbeeinflussung einen erhöhten Entwicklungs- und Zulassungsaufwand.

Aus der Bewertung der Konzeptgruppen und den sich daraus ergebenden relativen Bewertungen $r_{j}$ geht hervor, dass die Konzeptgruppe des Verschiebens starrer Komponenten am besten für variable Einlässe geeignet ist, vgl. Tabelle 4.17 und Abbildung 4.33. Deshalb wurde im weiteren Verlauf ein Konzept dieser Konzeptgruppe detailliert untersucht. 
Tabelle 4.17 Bewertung der Konzeptgruppen

\begin{tabular}{l|l|l|l}
\hline Kriterium & $\begin{array}{l}\text { Verschieben } \\
\text { starrer } \\
\text { Komponenten }\end{array}$ & $\begin{array}{l}\text { Verformen } \\
\text { elastischer } \\
\text { Oberflächen }\end{array}$ & $\begin{array}{l}\text { Grenzschicht- } \\
\text { beeinflussung }\end{array}$ \\
\hline $\begin{array}{l}\text { Aerodynamische Funktionalität } \\
\left(\boldsymbol{w}_{1}=0,267\right)\end{array}$ & + & ++ & ++ \\
\hline Integrierbarkeit $\left(\boldsymbol{w}_{2}=0,067\right)$ & + & - & o \\
\hline $\begin{array}{l}\text { Masse und Beanspruchungswiderstand } \\
\left(\boldsymbol{w}_{3}=0,133\right)\end{array}$ & $\mathrm{o}$ & - & + \\
\hline Sicherheit $\left(\boldsymbol{w}_{4}=0,267\right)$ & ++ & + & - \\
\hline $\begin{array}{l}\text { Zuverlässigkeit und Lebenswegkosten } \\
\left(\boldsymbol{w}_{5}=0,200\right)\end{array}$ & + & - & + \\
\hline $\begin{array}{l}\text { Entwicklungs- und Produktionskosten } \\
\left(\boldsymbol{w}_{6}=0,067\right)\end{array}$ & + & - & - \\
\hline Relative Bewertung $r_{j}$ & $\mathbf{0 , 7 8 3}$ & 0,183 & 0,633 \\
\hline
\end{tabular}

Zellwerte: Erfüllung des Kriteriums ist sehr gut ++, gut + , durchschnittlich o, schlecht - oder sehr schlecht --

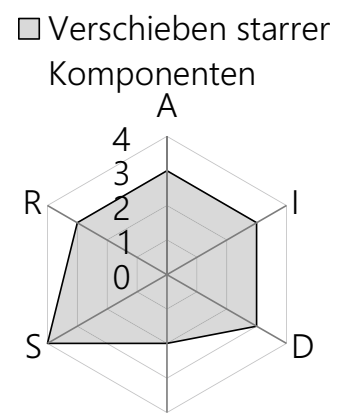

W

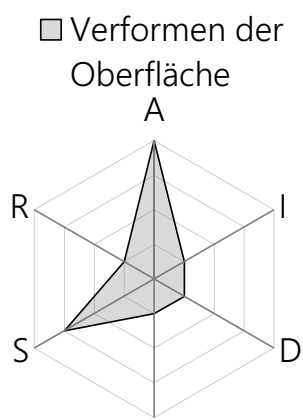

W

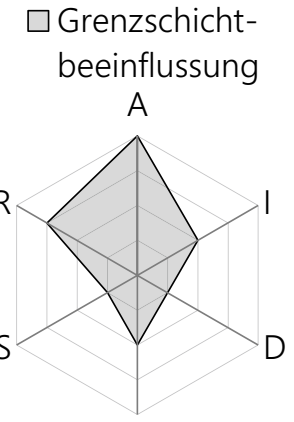

W

Aerodynamische Funktionalität (A)

Integrierbarkeit (I)

Zuverlässigkeit und Lebenswegkosten (R)

Entwicklungs- und Produktionskosten (D)

Sicherheit (S)

Masse und Beanspruchungswiderstand (W)

Abbildung 4.33 Bewertung der Konzeptgruppen 


\subsection{Detaillierte Konzeptuntersuchungen}

Basierend auf den zuvor beschriebenen Untersuchungen wurde die Konzeptgruppe für variable Einlässe durch Verschieben starrer Segmente als die bestgeeignete identifiziert. Nachfolgend wird erläutert, wie aus dieser Konzeptgruppe das umzusetzende Konzept hergeleitet, strukturell dimensioniert, in einer CADUmgebung modelliert, in einem Prototyp umgesetzt und seine strukturelle Stabilität mittels Vogelschlaganalyse vorläufig gezeigt wurde.

\subsubsection{Konzeptbeschreibung und Herleitung}

Das umzusetzende Konzept ist in Abbildung 4.34 dargestellt. Bei diesem Konzept verschiebt eine elektrisch betriebene Aktorik A einen Stellring $\mathrm{R}$ parallel zur Triebwerksrotationsachse. Die Bewegung des Stellrings $\mathrm{R}$ wird von einer Schienenführung $\mathrm{F}$ getragen und geleitet, wodurch die Aktorik entlastet wird. Durch die Bewegung des Stellrings $\mathrm{R}$ wird die axial und über den Umfang segmentierte umströmte Kontur des Einlasses variiert. Die axiale Unterteilung erfolgt in je vier umströmte Segmente auf der Außenseite S1, S3, S5 und S7 sowie auf der Innenseite S2, S4, S6 und S8. Axial benachbarte Segmente sind gelenkig miteinander verbunden. Die Segmente S1 und S2 sind zudem gelenkig mit dem statischen Teil des Einlasses gekoppelt. Über den Umfang erfolgt eine Unterteilung der umströmten Segmente in $15^{\circ}$-Sektoren. Die Querschnittsvariation wird dabei durch ein teilweises Überlappen der Segmente ermöglicht, vgl. Abbildung 4.11 in Abschnitt 4.2.

Der Einlass wird als zusammenhängende Baugruppe auf axialer Höhe der Fanebene an die restliche Gondel montiert. Hierfür ist zwischen den Segmenten S1 bzw. S2 und der Fanebene ein starrer Bereich vorgesehen. Die Montage des Einlasses kann beispielsweise durch eine Flanschverbindung zwischen dem starren Bereich des Einlasses und der Triebwerksgondel erfolgen. Dies wird in dieser Arbeit nicht detailliert betrachtet und alle unbeweglichen Komponenten von Einlass und Gondel werden als statische Gondel G zusammengefasst. Weiterhin müssen in den starren Bereich des Einlasses auch die funktionalen und mechanischen Schnittstellen zwischen Einlass und restlicher Gondel integriert werden. Zudem werden die Aktorik A und die Schienenführung F des Stellrings R in besagtem Bereich gelagert. 

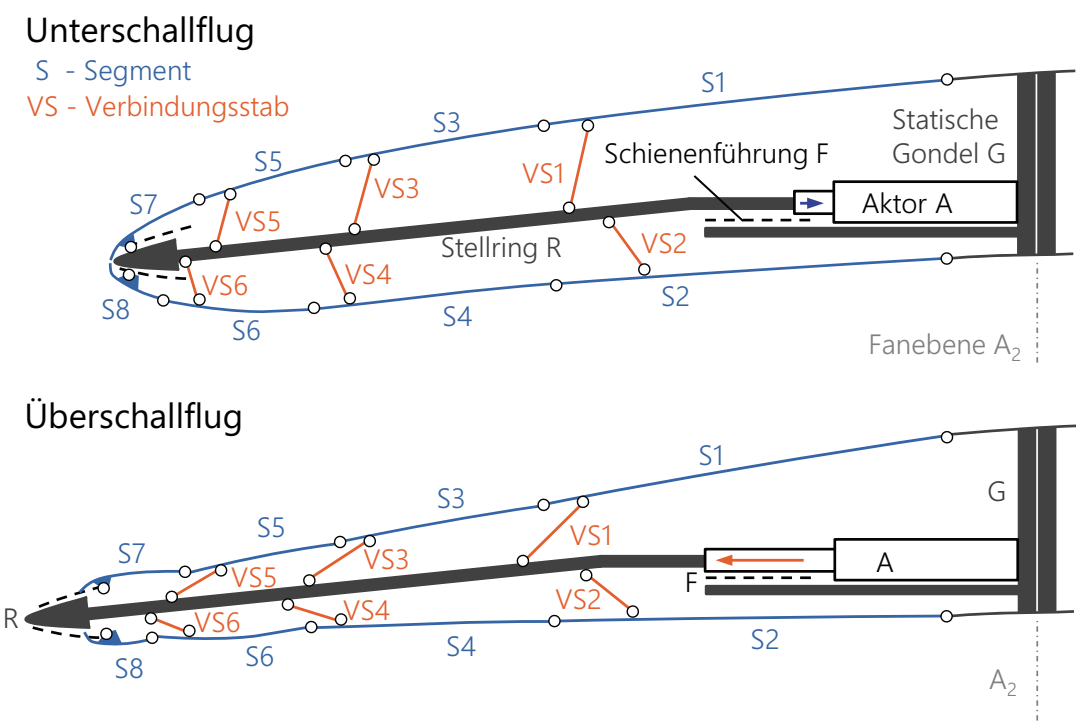

Abbildung 4.34 Prinzipielle Darstellung des Konzepts

Der Stellring $\mathrm{R}$ ist über seine axiale Erstreckung teilweise als geschlossener Ring und teilweise segmentiert ausgeführt, vgl. Abbildung 4.50. Im Bereich der Vorderkante ist der Stellring ein über den Umfang geschlossener Ring, der im Folgenden als Vorderkantenring bezeichnet wird, vgl. Abbildung 4.35.

Über die Verbindungsstäbe VS1 bis VS8 ist der Stellring R mit den jeweiligen umströmten Außensegmenten des Konzeptes S1 bis S8 verbunden. Bei den Segmenten S1 bis S6 erfolgt die Lagerung der Verbindungsstäbe VS1 bis VS6 beidseitig gelenkig. Bei den Segmenten S7 und S8 sind die Verbindungstäbe VS7/8 fest in die Segmente integriert. Die Verbindung zum Stellring R erfolgt bei den Segmenten S7/8 über Gelenkbolzen BRV7/8, die fest mit VS7/8 verbunden sind und innerhalb der Vorderkante des Stellrings R entlanggleiten können. Um dies zu ermöglichen, sind Schienen im Vorderkantenring integriert. Die gelenkigen Verbindungen zwischen den Komponenten des variablen Konzepts werden in allen Fällen über Bolzenverbindungen realisiert. 


\section{Unterschallflug}
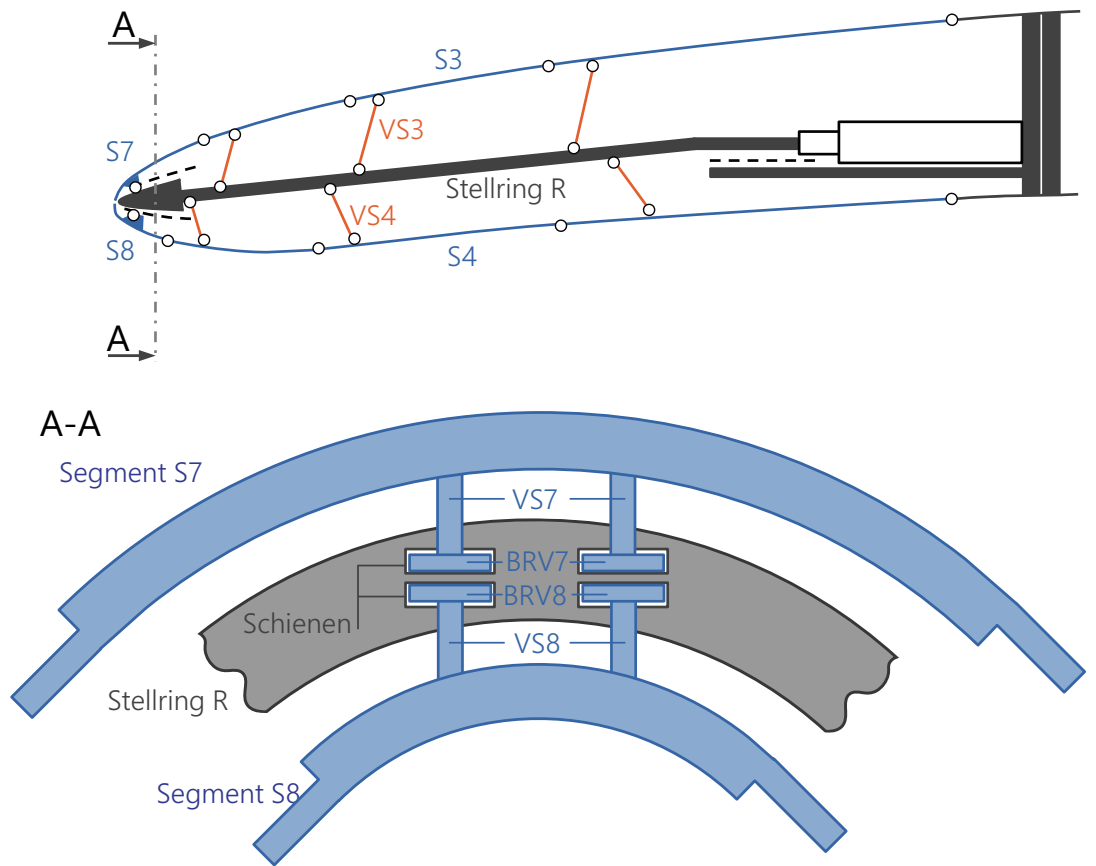

Abbildung 4.35 Funktionsprinzip gleitender Gelenke im Vorderkantenring

Eisbildung wird durch elektrische Enteisungsmatten verhindert. Diese erstrecken sich über die gesamte umströmte Einlassoberfläche, um auch Eisbildung zwischen den Segmenten benachbarter Sektoren zu verhindern [300]. Vom Fan ausgehende Lärmemissionen werden durch herkömmliche Akustikauskleidungen in den Segmenten S2 und S4 minimiert. Eine umfassende Auflistung der Bauteile des Konzepts ist Tabelle 4.18 zu entnehmen. Bauteile, deren Anzahl in Klammern steht, sind Bestandteil eines anderen Bauteils. 
Tabelle 4.18 Bauteilliste des Konzepts

\begin{tabular}{|c|c|c|c|}
\hline ID & Bezeichnung & Beschreibung & Anzahl \\
\hline G & Statische Gondel & $\begin{array}{l}\text { Statische Komponenten der Triebwerksgondel, } \\
\text { einschließlich des statischen Einlassteils sowie } \\
\text { Schnittstellen zwischen Einlass und Gondel }\end{array}$ & 1 \\
\hline A & Aktorik & $\begin{array}{l}\text { Antriebssystem des variablen Einlasses, } \\
\text { einschließlich Stelltrieben, Motoren, Sensorik, } \\
\text { Steuerelektronik, Feststellmechanismen und Kabeln }\end{array}$ & 6 \\
\hline $\mathrm{F}$ & Führungsschienen & $\begin{array}{l}\text { Entlastung der Aktorik A durch Tragen und Führen } \\
\text { des Stellrings R }\end{array}$ & 24 \\
\hline $\mathrm{R}$ & Stellring & $\begin{array}{l}\text { Umlaufende Struktur; durch Schienenführung F } \\
\text { gelagert; axiale Position von Aktorik A gesteuert; } \\
\text { über Verbindungsstäbe VS1 bis VS6 und Gelenke mit } \\
\text { überströmten Segmenten S1 bis S6 verbunden; } \\
\text { Vorderkante ringförmig mit integrierten Schienen; } \\
\text { bildet im Überschallbetrieb Teil der Einlasslippe; über } \\
\text { gleitende Gelenke mit Segmenten S7/8 verbunden }\end{array}$ & 1 \\
\hline S1 & Segment 1 & $\begin{array}{l}\text { Überströmtes Segment auf der Einlassaußenseite; } \\
\text { gelenkig mit statischer Gondel G, Segment S3 sowie } \\
\text { Verbindungsstab VS1 verbunden }\end{array}$ & 24 \\
\hline $\mathrm{S} 2$ & Segment 2 & $\begin{array}{l}\text { Überströmtes Segment auf der Einlassinnenseite; } \\
\text { gelenkig mit statischer Gondel G, Segment S4 sowie } \\
\text { Verbindungsstab VS2 verbunden }\end{array}$ & 24 \\
\hline S3 & Segment 3 & $\begin{array}{l}\text { Überströmtes Segment auf der Einlassaußenseite; } \\
\text { gelenkig mit Segmenten S1 und S5 sowie } \\
\text { Verbindungsstab VS3 verbunden }\end{array}$ & 24 \\
\hline S4 & Segment 4 & $\begin{array}{l}\text { Überströmtes Segment auf der Einlassinnenseite; } \\
\text { gelenkig mit Segmenten S2 und S6 sowie } \\
\text { Verbindungsstab VS4 verbunden }\end{array}$ & 24 \\
\hline S5 & Segment 5 & $\begin{array}{l}\text { Überströmtes Segment auf der Einlassaußenseite; } \\
\text { gelenkig mit Segmenten S3 und S7 sowie } \\
\text { Verbindungsstab VS5 verbunden }\end{array}$ & 24 \\
\hline S6 & Segment 6 & $\begin{array}{l}\text { Überströmtes Segment auf der Einlassinnenseite; } \\
\text { gelenkig mit Segmenten S4 und S8 sowie } \\
\text { Verbindungsstab VS6 verbunden }\end{array}$ & 24 \\
\hline S7 & Segment 7 & $\begin{array}{l}\text { Überströmtes Segment auf der Einlassaußenseite; } \\
\text { gelenkig mit Segment S5 verbunden; beinhaltet } \\
\text { Verbindungsstab VS7 und Gelenkbolzen BRV7 für } \\
\text { gleitende Gelenkverbindung mit Vorderkantenring R }\end{array}$ & 24 \\
\hline
\end{tabular}


Tabelle 4.18 (Fortsetzung)

\begin{tabular}{|c|c|c|c|}
\hline ID & Bezeichnung & Beschreibung & Anzahl \\
\hline S8 & Segment 8 & $\begin{array}{l}\text { Überströmtes Segment auf der Einlassinnenseite; } \\
\text { gelenkig mit Segment S6 verbunden; beinhaltet } \\
\text { Verbindungsstab VS8 und Gelenkbolzen BRV8 für } \\
\text { gleitende Gelenkverbindung mit Vorderkantenring R }\end{array}$ & 24 \\
\hline VS1 & Verbindungsstab 1 & $\begin{array}{l}\text { Stab; gelenkig mit Stellring R und Segment S1 } \\
\text { verbunden }\end{array}$ & 48 \\
\hline VS2 & Verbindungsstab 2 & $\begin{array}{l}\text { Stab; gelenkig mit Stellring R und Segment S2 } \\
\text { verbunden }\end{array}$ & 48 \\
\hline VS3 & Verbindungsstab 3 & $\begin{array}{l}\text { Stab; gelenkig mit Stellring R und Segment S3 } \\
\text { verbunden }\end{array}$ & 48 \\
\hline VS4 & Verbindungsstab 4 & $\begin{array}{l}\text { Stab; gelenkig mit Stellring R und Segment S4 } \\
\text { verbunden }\end{array}$ & 48 \\
\hline VS5 & Verbindungsstab 5 & $\begin{array}{l}\text { Stab; gelenkig mit Stellring R und Segment S5 } \\
\text { verbunden }\end{array}$ & 48 \\
\hline VS6 & Verbindungsstab 6 & $\begin{array}{l}\text { Stab; gelenkig mit Stellring R und Segment S6 } \\
\text { verbunden }\end{array}$ & 48 \\
\hline VS7 & Verbindungsstab 7 & $\begin{array}{l}\text { Stab; fest mit Segment S7 und Gelenkbolzen BRV7 } \\
\text { verbunden }\end{array}$ & $(48)$ \\
\hline VS8 & Verbindungsstab 8 & $\begin{array}{l}\text { Stab; fest mit Segment S8 und Gelenkbolzen BRV8 } \\
\text { verbunden }\end{array}$ & $(48)$ \\
\hline BSG1 & Gelenkbolzen SG1 & $\begin{array}{l}\text { Gelenkbolzen zwischen Segment S1 und der } \\
\text { statischen Gondel G }\end{array}$ & 48 \\
\hline BSG2 & Gelenkbolzen SG2 & $\begin{array}{l}\text { Gelenkbolzen zwischen Segment S2 und der } \\
\text { statischen Gondel G }\end{array}$ & 48 \\
\hline BS13 & Gelenkbolzen S13 & Gelenkbolzen zwischen Segment S1 und Segment S3 & 48 \\
\hline BS24 & Gelenkbolzen S24 & Gelenkbolzen zwischen Segment S2 und Segment S4 & 48 \\
\hline BS35 & Gelenkbolzen S35 & Gelenkbolzen zwischen Segment S3 und Segment S5 & 48 \\
\hline BS46 & Gelenkbolzen S46 & Gelenkbolzen zwischen Segment S4 und Segment S6 & 48 \\
\hline BS57 & Gelenkbolzen S57 & Gelenkbolzen zwischen Segment S5 und Segment S7 & 48 \\
\hline BS68 & Gelenkbolzen S68 & Gelenkbolzen zwischen Segment S6 und Segment S8 & 48 \\
\hline BSV1 & Gelenkbolzen SV1 & $\begin{array}{l}\text { Gelenkbolzen zwischen Segment S1 und } \\
\text { Verbindungsstab VS1 }\end{array}$ & 48 \\
\hline BSV2 & Gelenkbolzen SV2 & $\begin{array}{l}\text { Gelenkbolzen zwischen Segment S2 und } \\
\text { Verbindungsstab VS2 }\end{array}$ & 48 \\
\hline
\end{tabular}


Tabelle 4.18 (Fortsetzung)

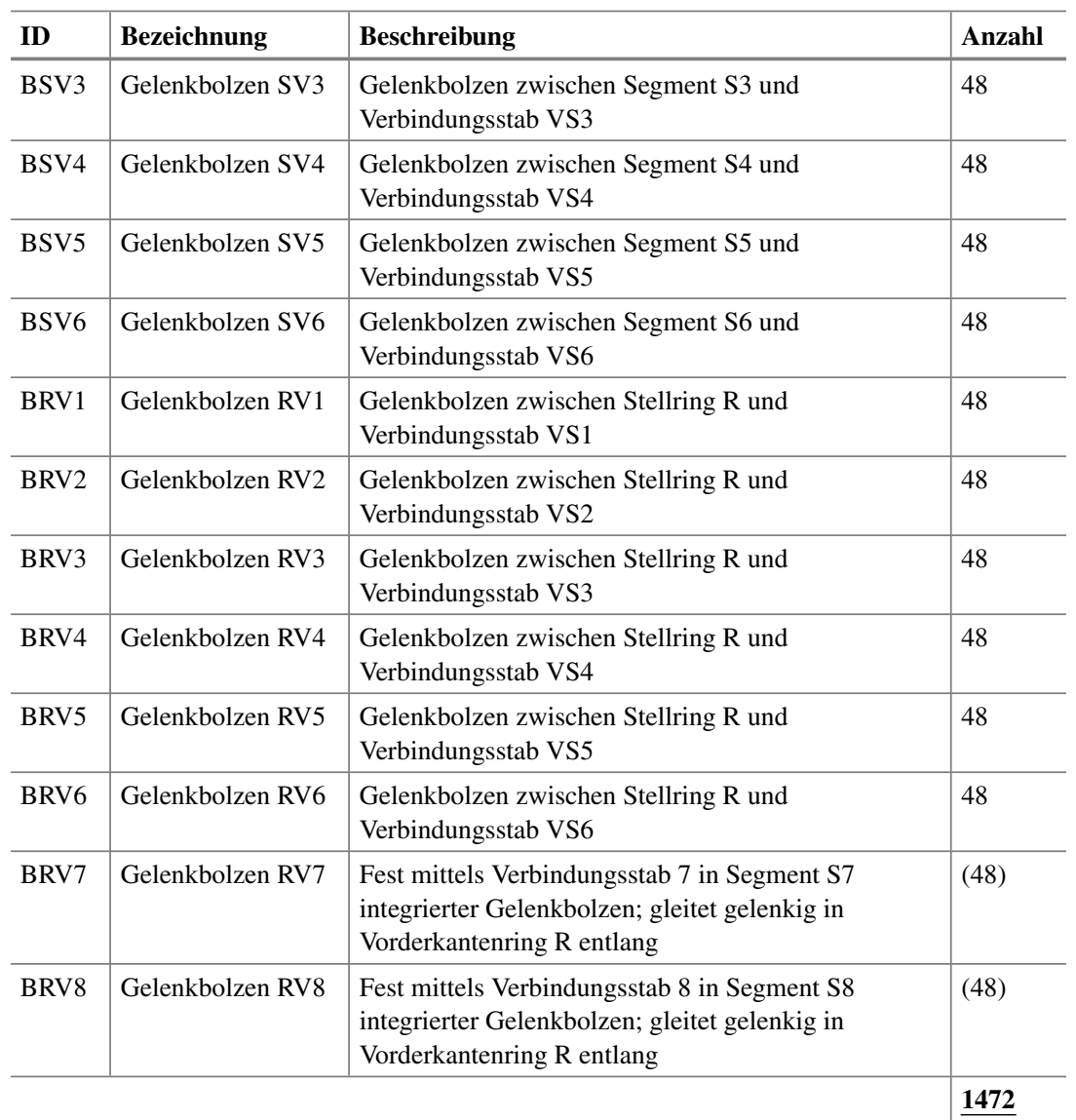




\section{Funktionsweise}

Das Konzept kann zwei Zustände annehmen. Im ersten Zustand wird eine Geometrie für den Unterschallflug eingestellt. Dieser Zustand repräsentiert zugleich die Nominalstellung des Systems, welche im unbelasteten Fall, aber auch im Fehlerfall, umgesetzt werden soll. Der zweite Zustand realisiert eine Geometrie, die für den Überschallflug geeignet ist. Durch axiales Verfahren des Stellrings kann zwischen den Zuständen gewechselt werden.

Beim Verfahren des Aktors A aus dem Unterschallzustand wird der Stellring R entgegen der Flugrichtung axial verschoben. Dadurch verändert sich die Lage der Verbindungsstäbe VS1 bis VS6, wodurch wiederum die umströmten Segmente S1 bis S6 in die gewünschte Position gezogen werden. Durch das axiale Vorfahren des Stellrings gleiten zudem die Segmente S7 und S8 mit Hilfe der Bolzengelenke entlang der Schienen im Vorderkantenring $\mathrm{R}$ an diesem entlang. Dadurch wird der Vorderkantenring zum vordersten Teil der Einlasslippe und bildet gemeinsam mit den umströmten Segmenten S1 bis S8 die Einlassgeometrie während des Überschallbetriebs.

Für das Verfahren des Stellrings wird ein elektrisch betriebenes Aktorsystem A verwendet. Dieses kann Synergien mit dem elektrischen Enteisungssystem nutzen [300]. Ein Umwandeln der Geometrie von Unterschall zu Überschall darf sicherheitsbedingt nur im stationären Reiseflug erfolgen, während die Änderung von Überschall- zu Unterschallgeometrie während jeder Flugphase erfolgen darf.

Bei Fehlern oder dem Ausfall von Teilsystemen sind Vorkehrungen zu treffen, die gefährliche Fehlerfolgen verhindern. Relevante Fehlerfälle sind unter anderem das ungewollte Ausfahren des Aktors und somit eine ungewollte Verstellung der Geometrie während des Startfalls. Da dieser Fehler gefährliche Folgen haben kann, sind Gegenmaßnahmen zu ergreifen, wie beispielsweise ein vom Aktorkontrollsystem unabhängiger Feststellmechanismus für die Aktoren, vgl. Kazula et al. [299] und Abbildung 4.15. Die Position des Aktors und somit des Stellrings wird deshalb durch ein unabhängig angesteuertes Bremssystem gegen ungewolltes Verstellen gesichert. Beim Ausfall des Aktorsystems, beispielsweise beim Fehlen elektrischer Energie zum Verstellen, sollte der variable Einlass die Geometrie für den Unterschall einnehmen. Erfolgt dies nicht automatisch durch die wirkenden Strömungslasten, kann hierfür ein zusätzlicher Federmechanismus zum Zurückfahren des Stellrings R eingesetzt werden.

\section{Statische und kinematische Bestimmtheit}

Das Konzept als mechanisches System muss statisch und kinematisch eindeutig bestimmt sein. Dies bedeutet, dass jeder Position des Aktors genau eine Position der Systemkomponenten und somit genau eine Einlassgeometrie zugeordnet sein muss [356, S. 90]. Die notwendige Bedingung hierfür ist ein statisch 
bestimmtes System, welches mit Hilfe eines Abzählkriteriums nachgewiesen wird [356, S. 91-106]. Nach diesem muss die Anzahl der ungebundenen Bewegungsfreiheitsgrade aller Systemkomponenten $f$ gleich der Anzahl der Wertigkeiten der Bindungselemente oder Lagerungen des Systems $n$ sein [356, S. 91]. Als hinreichende Bedingung für die kinematische Bestimmtheit kann ein Polplan herangezogen werden oder direkt gezeigt werden, dass das System unbeweglich ist [356, S. 91-106], [357, S. 142-147].

Nachfolgend wird die statische und kinematische Bestimmtheit des Konzepts in der Schnittebene aus Abbildung 4.34 gezeigt. Die Starrkörper des Systems haben in dieser ebenen Betrachtungsweise jeweils drei Freiheitsgrade:

- horizontale Verschiebung,

- vertikale Verschiebung und

- Rotation in der Ebene.

Das Konzept beinhaltet 17 Starrkörper und somit insgesamt 51 Bewegungsfreiheitsgrade $f$, vgl. obere Hälfte von Abbildung 4.36.

Freiheitsgrad des ungebundenen Systems

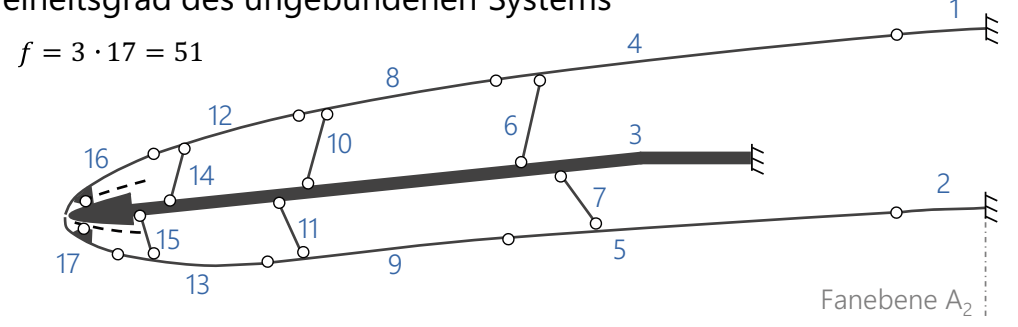

Zahl der Bindungen des Systems

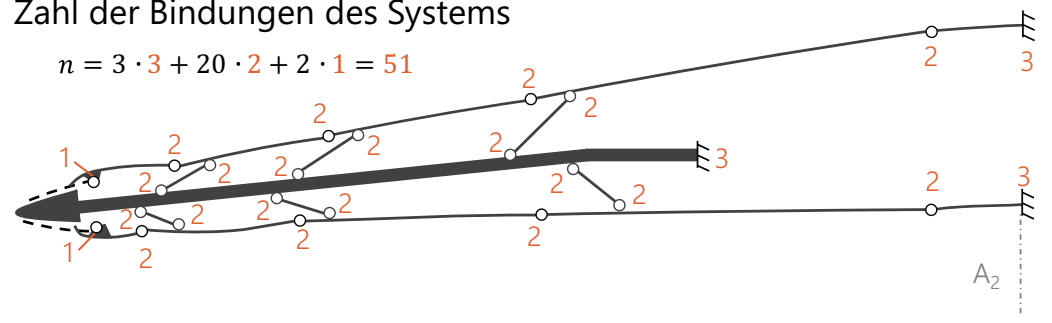

Abbildung 4.36 Freiheitsgrade und Lagerwertigkeiten des Konzepts 
Die Wertigkeit einer Bindung ist entsprechend der Anzahl der Freiheitsgrade, die sie aus dem System entfernt. Die Aktorik A und die Führungsschienen F stellen für jeden Zustand des Systems genau eine Position des Stellrings sicher. Diese Positionssicherung wird im Folgenden als feste Einspannung mit einer Wertigkeit von 3 modelliert. Auch der statische Teil des Einlasses ist durch die Flanschverbindung zur restlichen Gondel fest eingespannt. Die Schienen im Vorderkantenring realisieren ein Dreh-Schiebe-Gelenk mit einer Wertigkeit von 1, da sowohl eine Rotation des Segmentes S7 bzw. S8 möglich ist, als auch ein Abgleiten entlang der Schiene [356, S. 106]. Alle weiteren Gelenkbolzenverbindungen stellen Drehgelenke mit einer Wertigkeit von 2 dar [356, S. 106]. Wie aus der unteren Hälfte von Abbildung 4.36 hervorgeht, beträgt die Summe der Wertigkeiten aller unabhängigen Bindungen $n$ des Konzepts somit insgesamt 51. Folglich ist das System statisch bestimmt. Weiterhin ist aus der Anschauung feststellbar, dass das abgebildete System unbeweglich ist. Daraus resultiert, dass das gebundene System keinen Freiheitsgrad hat und deshalb kinematisch eindeutig bestimmt ist.

\section{Umsetzung der Kinematik über Stellring und Verbindungsstäbe}

Das Verhalten der Kinematik und somit die vom Konzept umsetzbaren Geometrien werden durch Verfahren des Stellrings R gesteuert. Der Stellring R ist eine umlaufende Struktur, die durch die Schienenführung $\mathrm{F}$ gehalten und deren axiale Position von der Aktorik A gesteuert wird. Über die Verbindungsstäbe VS1 bis VS6 und die zugehörigen Bolzengelenke ist er mit den überströmten, konturgebenden Segmenten S1 bis S6 des Konzepts verbunden, vgl. Abbildung 4.37. Die Segmente S1 bis S6 sind über je zwei Verbindungsstäbe mit dem Stellring verbunden. Diese doppelte Ausführung resultiert in einer erhöhten Sicherheit durch Redundanz im Versagensfall einer der genannten Komponenten.

Der Stellring beinhaltet zudem den Vorderkantenring, der während des Überschallreiseflugs einen Teil der Einlasslippe bildet. Über integrierte Schienen im besagten Vorderkantenring ist der Stellring direkt mit den Segmenten S7 und S8 verbunden. Während langsamer Fluggeschwindigkeiten in Bodennähe ist der Vorderkantenring im Inneren des Einlasses verstaut. Dadurch ist während der zugehörigen sicherheitskritischen Phasen durch die Segmente S7 und S8 eine aerodynamisch bessere Geometrie im Vorderkantenbereich einstellbar. Um die Masse des Stellrings zu minimieren, ist er nur an den Lagerungsstellen der Verbindungsstäbe und in der Vorderkante als durchgängiger Ring ausgeführt. In den Bereichen zwischen den Ringen wird er durch einen Stab pro Sektor umgesetzt.

Durch das axiale Verschieben des Stellrings $\mathrm{R}$ ändert sich die Lage der Verbindungsstäbe und somit auch die jeweilige Position der damit verbundenen 


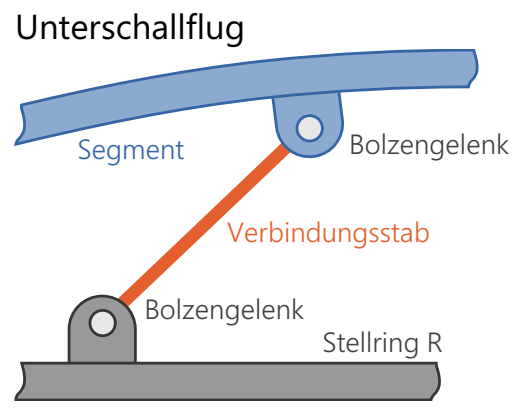

Überschallflug

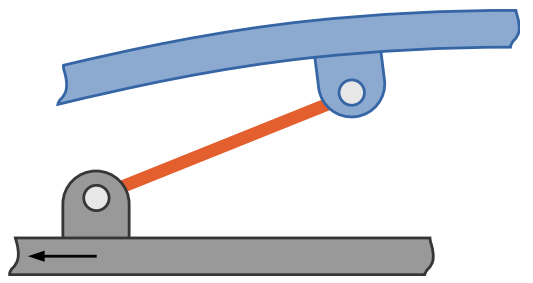

Abbildung 4.37 Gelenkverbindungen über Bolzen

Segmente, die in die gewünschten Positionen gezogen werden. Die vom Konzept umsetzbaren Geometrien sind daher stark abhängig von der nominalen Lage und der Länge der Verbindungsstäbe.

Die Umwandlung der axialen Stellringbewegung in die gewünschte Bewegung des Aufhängungspunktes des zugehörigen Segmentes wird dabei insbesondere vom Winkel $\alpha$ zwischen dem Verbindungsstab und dem Stellring beeinflusst, vgl. Abbildung 4.38. Dabei dürfen keine „Pendelsituationen“ auftreten. In diesen könnten die verbundenen Stäbe und Segmente sowohl in die eine Richtung als auch in die andere Richtung ausschlagen. Dies wäre bei einem gestreckten Winkel $\alpha$ der Fall, weshalb im vorliegenden Konzept generell spitze Winkel $\alpha$ realisiert werden. Zudem werden gestreckte Winkel zwischen den Segmenten und den zugehörigen Verbindungsstäben vermieden.

Die Längen und Lagerungspositionen der Verbindungsstäbe sowie die axialen Positionen, an denen die Einlasskontur in Segmente unterteilt wird, wurden iterativ bestimmt und gehen aus Tabelle A.1 in Anhang A.1.2 bzw. Tabelle A.7 in Anhang A.6.4 hervor. Diese Daten können in einer späteren Entwicklungsphase auch mathematisch bestimmt und strömungsmechanisch optimiert werden.

\section{Herleitung des Konzeptes}

Die Herleitung des Konzeptes basiert vorrangig auf den nachfolgenden Anforderungen für variable Einlasskonzepte:

- Gewährleistung der Sicherheit beim Start und im Steigflug,

- hohe Effizienz im Reiseflug sowie

- hohe Zuverlässigkeit. 
Verbindungsstablängenbestimmung
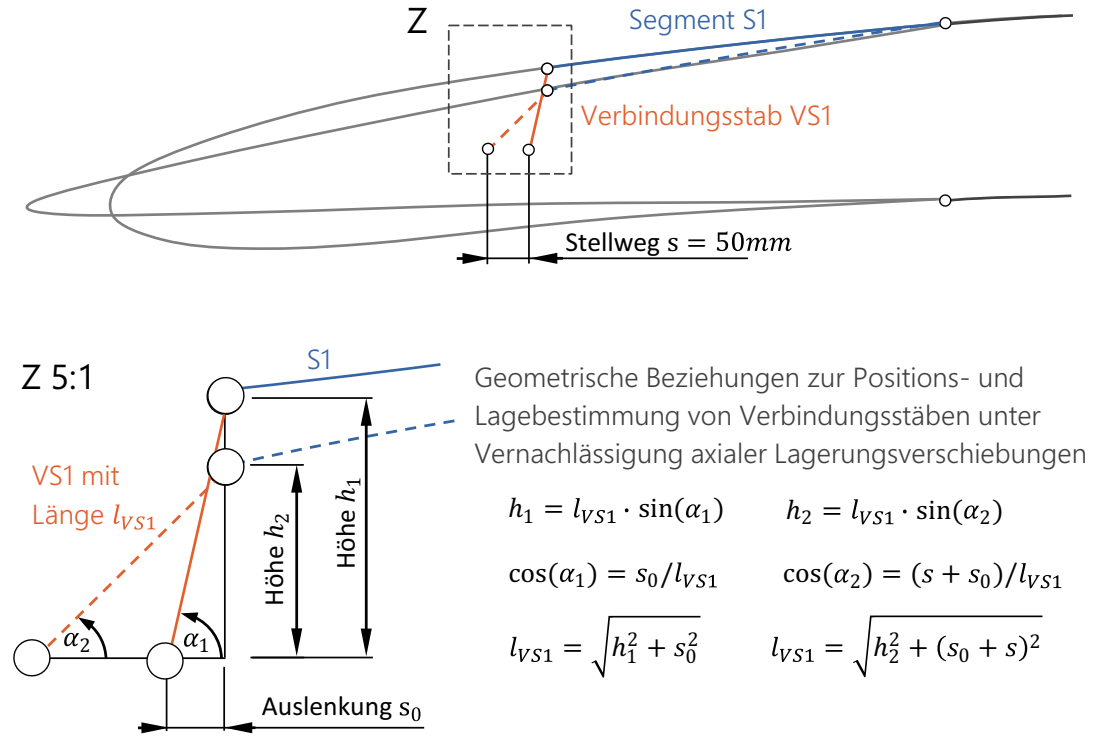

Abbildung 4.38 Ermittlung der Verbindungsstablängen am Beispiel von Verbindungsstab VS1

Ausreichende Sicherheit im Startfall und im Steigflug ist durch Sicherstellen einer geeigneten Geometrie von hoher Güte erreichbar. Eine Geometrie mit hoher aerodynamischer Güte kann durch die Realisierung einer glatten Oberfläche mit minimalen Stufen und Spalten erreicht werden. Auch die Effizienz bei schnellen Fluggeschwindigkeiten hängt neben der Geometriedicke und der Masse von der Glattheit der Oberfläche ab.

Bei der Überführung verschiedener Einlassgeometrien durch Verschieben fester Segmente kann nur für einen Zustand eine glatte Oberfläche erzeugt werden. Dies gilt sowohl radial, vgl. Abbildung 4.8 in Abschnitt 4.2.4, als auch axial, vgl. Abbildung 4.39. Zur Gewährleistung der Sicherheit im Startfall und beim Steigflug stellt das umzusetzende Konzept während dieser kritischen Flugphasen im Unterschallbereich [298] eine annähernd ideale, glatte Geometrie bereit.

Für die Glattheit der Überschallgeometrie ist die Anzahl der axialen Segmente entscheidend. Durch eine geringe Anzahl axialer Segmente entstehen bei der 
Glatte Geometrie für den Unterschallflug

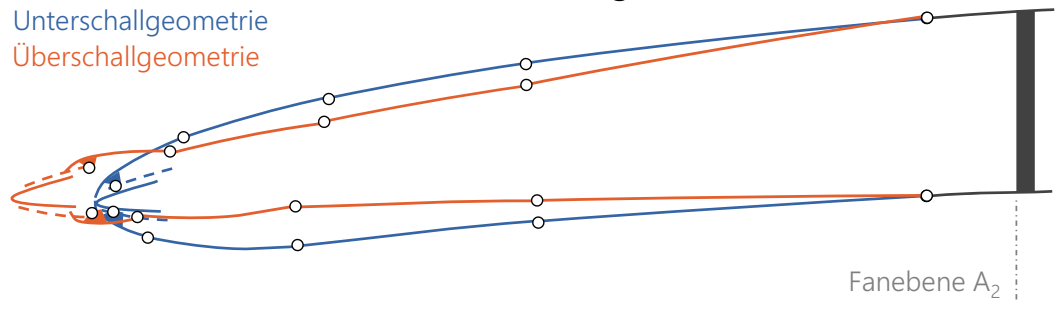

Glatte Geometrie für den Überschallflug

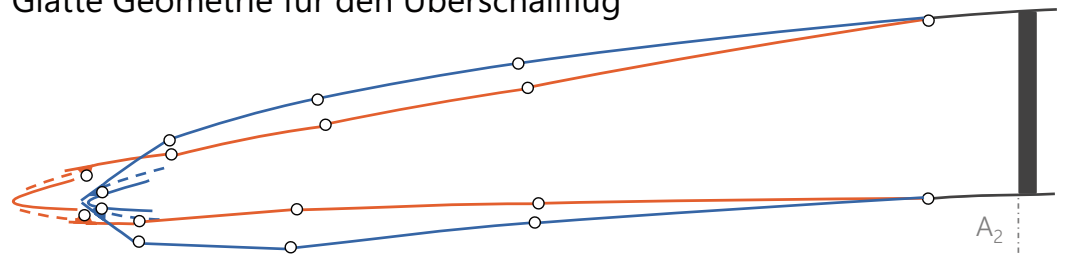

Abbildung 4.39 Möglichkeiten axialer Geometrieglattheit

Überschallgeometrie größere Stufen. Diese können Verdichtungsstöße und Strömungsablösungen zur Folge haben sowie gegebenenfalls die Effizienz und den Luftwiderstand des Einlasses beeinträchtigen. Wie zuvor beschrieben, ist der Einlass axial in je vier umströmte Segmente auf der Außenseite S1, S3, S5 und S7 sowie auf der Innenseite S2, S4, S6 und S8 unterteilt. Eine Unterteilung in mehr Abschnitte würde die Glattheit der Geometrie verbessern, während sie eine höhere Komplexität des Konzepts zur Folge hätte. Die Festlegung auf vier axiale Segmente stellt somit einen Kompromiss aus Effizienz der umsetzbaren Kontur im Überschallreiseflug sowie Komplexität und somit Zuverlässigkeit durch die dafür verwendete Komponentenanzahl dar.

Die axial benachbarten Segmente eines Sektors sind über jeweils zwei Bolzengelenkverbindungen miteinander verbunden, vgl. Abbildung 4.40. Die redundant ausgeführten Bolzenverbindungen ermöglichen eine hohe Genauigkeit bei der Umsetzung der kleinen Bewegungen der Gelenke und gewährleisten eine größere Sicherheit im Versagensfall eines Bolzens.

Von einer signifikanten Längenvariation des Einlasses mittels Teleskopprinzip wird aufgrund der großen erforderlichen Masse und Komplexität abgesehen, vgl. Genßler [314] und Wöllner [315]. Sollte im Verlauf späterer Entwicklungsphasen für den Startfall ein deutlich kürzerer Einlass als notwendig identifiziert werden, 
Variante ohne Aussparung

Unterschallflug

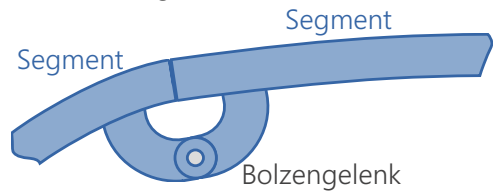

Überschallflug

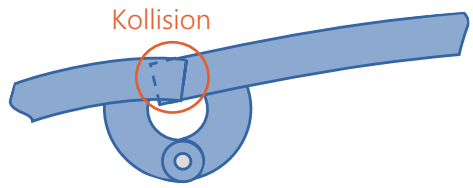

Variante mit Aussparung

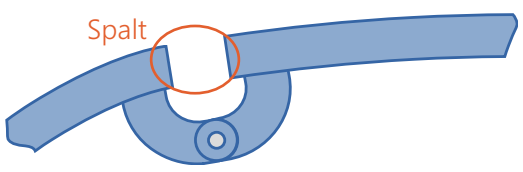

Abbildung 4.40 Kollision fester Segmente beim Verstellen

müsste das gewählte Konzept angepasst werden. Dies kann beispielsweise durch eine Verlängerung des Vorderkantenrings $\mathrm{R}$ und eine Verkürzung der Segmente S1 bis S4 realisiert werden [315, S. 104].

Die Variation des Querschnitts in verschiedenen Ebenen des Einlasses, beispielsweise in der Kehle und im Diffusor, wird durch die radiale Segmentierung in Sektoren mit teilweiser Überlappung ermöglicht. Die radiale Segmentierung und die Überlappungen haben zur Folge, dass die Geometrie einen Polygonquerschnitt an Stelle eines idealen Kreisquerschnitts realisiert, vgl. Abbildung 4.8. Die Größe der Abweichung von einem idealen Kreisquerschnitt hängt vorrangig von der Sektorgröße ab. Der Einfluss dieser Abweichung auf die Einlassströmung ist zu untersuchen. Die Abweichungen können zum einen kleine Ungleichförmigkeiten der Strömung in der Ebene des Fans nach sich ziehen. Zum anderen können sie bewirken, dass potenzielle Ablöseblasen nicht über einen Sektor hinauswachsen und somit keine kritische Größe erreichen [358]. Kleine Sektoren resultieren in einer geringeren Abweichung und kleineren Segmenten S1 bis S8, die potenziell bei einem Vogelschlag verloren gehen könnten. Zudem können insbesondere im Bereich der Lippe potenziell entstehende Spalte zwischen Segmenten minimiert werden. Allerdings steigt gleichzeitig die Anzahl der Segmente über den Umfang. Bei einer Segmentgröße von $30^{\circ}$ würden 12 Umfangssegmente resultieren, bei $20^{\circ}$ wären es 18 , bei $15^{\circ}$ wären es 24 und bei $10^{\circ}$ wären es 36 . Unter 
Berücksichtigung der genannten Einflüsse wurden $15^{\circ}$-Sektoren gewählt. Weiterhin erzeugen die Segmentverlängerungen zur Überlappung radiale Stufen im Größenbereich von 1 bis $2 \mathrm{~mm}$, deren aerodynamische Auswirkungen in späteren Entwicklungsphasen zu untersuchen sind.

Beim Verschieben bzw. Rotieren benachbarter Segmente sind Kollisionen zwischen diesen Bauteilen zu vermeiden. Um dies zu erreichen, müssen auch Teile einzelner Segmente ausgespart werden, vgl. Abbildung 4.40. Dies führt zu Lücken bzw. Spalten zwischen Teilsegmenten. Insbesondere im Lippenbereich ist die Spaltgröße stark von der Anzahl der axialen Segmente sowie der Sektorengröße abhängig.

Diese Spalte können aerodynamische Auswirkungen auf den Einlass und das Flugzeug haben und zu zusätzlichen Belastungen, Eisansammlungen sowie Verschmutzungen innerhalb des Einlasses führen. Zudem können sie einen Einfluss auf die Lärmentstehung haben. In Abhängigkeit ihrer Gestaltung können die Spalte allerdings auch für die erforderliche Ventilation des Einlasses genutzt werden. Zudem könnten sie einen positiven aerodynamischen Einfluss haben, indem sie die Grenzschicht abscheiden und somit sowohl den Widerstand als auch die Wahrscheinlichkeit von Strömungsablösungen verringern [336]. Der Einfluss möglicher Spalte des abschließenden Konzepts, sollte im weiteren Verlauf der Auslegung untersucht werden.

Sollte der Einfluss der Spalte negativ sein, sind Gegenmaßnahmen zu ergreifen, die wiederum Auswirkungen auf die Komplexität und die Effizienz des Konzeptes haben können. Beispielsweise können die Spalte durch eingeklebte oder festgenietete Elastomer-Verbindungen abgedichtet werden, sodass weder Luft, Wasser, Hagel, Eis noch Schmutz in das Innere des Einlasses eindringen können. Beispielhafte Umsetzungen hierfür können Abbildung 4.41 entnommen werden.

Alternativ kann ein Druckgefälle vom Inneren des Einlasses hin zur umströmten Oberfläche umgesetzt werden. Dies erfordert einerseits Energie, die dem Triebwerk entnommen werden muss und somit vom potenziellen Effizienzgewinn durch den variablen Einlass abzuziehen ist. Andererseits könnte Luft durch die Spalte gezielt in die Grenzschicht der Einlassumströmung eingeblasen werden und somit Strömungsablösungen verhindern [336].

Insbesondere am Staupunkt im Bereich der Einlasslippe ist eine glatte Geometrie erforderlich, um Strömungsablösungen und deren potenzielle Folgen zu vermeiden. Jedoch sollte ein variabler Einlass die Geometrie im Lippenbereich variieren können, um sowohl im Unter- als auch im Überschallbereich hohe Effizienz gewährleisten zu können, vgl. Abschnitt 4.4.3.

Eine Variation der Lippendicke des Einlasses kann durch Rotieren der Lippensegmente in der Schnittebene oder durch Überlappen der Lippensegmente 
Axialspaltabdichtung

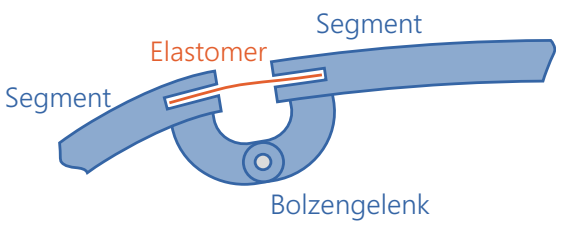

Umfangsspaltabdichtung

Variante A

Kleiner Querschnitt

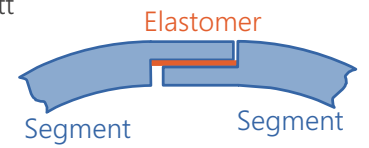

Mittlerer Querschnitt

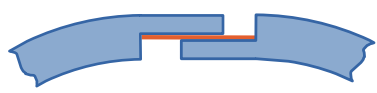

Großer Querschnitt

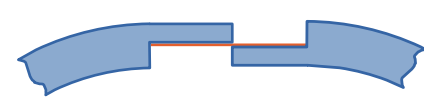

Variante B

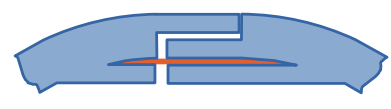

Abbildung 4.41 Möglichkeiten der Spaltabdeckung durch Elastomere

erreicht werden, vgl. Abbildung 4.42. Während beim Überlappen stets eine Stufe im Bereich der Vorderkante existiert, entsteht beim Rotieren ein Spalt. Die Größe des Spaltes ist unter anderem abhängig von der Sektorengröße und der Lagerung der Segmente. Beide Lösungen führen folglich zu einer negativen Beeinflussung der Strömung.

Unter anderem aus diesem Grund wird die Variante der Segmentrotation mit einem über den Umfang geschlossenen Vorderkantenring kombiniert. Der Vorderkantenring ist Bestandteil des Stellrings und wird mit diesem verschoben. Die Lippensegmente S7/S8 gleiten beim Verschieben des Stellrings auf diesem entlang. Die Spalte entstehen dadurch vom Staupunkt versetzt und sind etwa halb so groß wie bei der Variante ohne Vorderkantenring. Weiterhin kann die Kontur des Vorderkantenrings optimiert werden, um den Spalt zu minimieren. Durch 
Rotierende Lippensegmente

Seitenansicht Vorderansicht

Unterschallflug

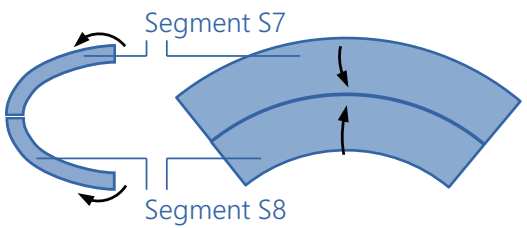

Überschallflug
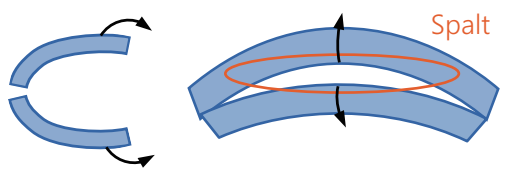

Überlappende Lippensegmente

Seitenansicht Vorderansicht
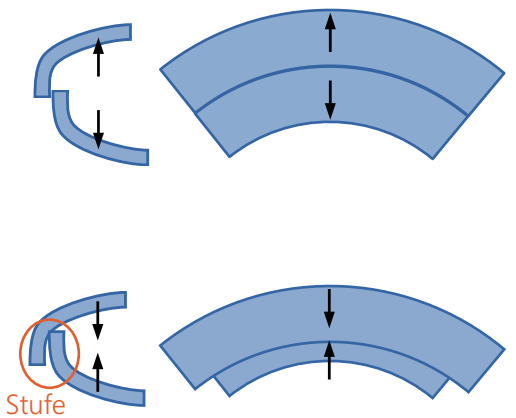

Abbildung 4.42 Spaltentstehung beim Drehen runder Körper

die Verwendung eines Vorderkantenrings wird für alle Zustände des Konzepts eine glatte Oberfläche im Bereich um den Staupunkt erreicht. Hierzu zählen die Zustände für Unterschall- und Überschallbetrieb, aber auch die Zwischenzustände beim Verfahren.

Bei entsprechender Auslegung des Stellmechanismus wäre es möglich, für jede Flugphase einen entsprechenden Zustand mit zugehöriger Geometrie umzusetzen. Insbesondere die Lage und Länge der Verbindungsstäbe ist hierbei ausschlaggebend. In späteren Entwicklungsphasen sollten die potenziell daraus resultierenden Vorteile bezüglich Effizienz und Betriebsverhalten mit dem erhöhten Entwicklungsaufwand und der zusätzlichen Komplexität des resultierenden Konzepts abgewogen werden.

Die Ausführung der Vorderkante als geschlossener Ring birgt über die strömungsmechanischen Verbesserungen hinaus weitere Vorteile in Bezug auf Sicherheit und Stabilität im Vogelschlagfall. Der über den Umfang geschlossene Ring kann dicker ausgeführt werden als drehbare Lippensegmente und ist widerstandsfähiger gegen Verformung und Bruch. Wie beschrieben, ermöglicht die Verwendung eines Vorderkantenrings die Variation der Einlasslippendicke. Dabei ist aus Gründen der Konstruktion und Fertigung eine Mindesthöhe und -dicke des Vorderkantenrings erforderlich. Beispielsweise müssen die Schienen für die gelenkige Gleitlagerung der Segmente S7 und S8 integriert werden 
können. Daraus resultiert eine minimal erforderliche Bauhöhe, worauf in der nachfolgenden Auslegung genauer eingegangen wird. Weiterhin ist bei der Verwendung eines Vorderkantenrings keine relevante Änderung des Eintrittsquerschnittes möglich, was bei der Festlegung der geeigneten Geometrien und der anschließenden Konzeptgruppenbewertung in Abschnitt 4.4 berücksichtigt wurde.

Eine Variation des Vorderkantenquerschnitts wäre mit einer über den Umfang segmentierten Vorderkante möglich. Jedoch werden bei gleichzeitiger Umsetzung einer Lippendickenvariation komplexe Abdichtungen notwendig, um der Entstehung großer Spalte entgegenzuwirken. Bei der Verwendung starrer Abdichtsegmente müssten diese über Kardan- oder Kugelgelenke realisiert werden [314, S. 87-90]. Bei Nutzung elastischer Abdichtungen wäre das elastische Material starken Belastungen in mehrere Richtungen ausgesetzt. Dies würde sich zusätzlich negativ auf die Lebensdauer auswirken. Beide Lösungen wären bei einer geringfügig günstigeren Geometrie aufgrund ihrer Komplexität im regulären Betrieb und insbesondere im Vogelschlagfall deutlich schadensanfälliger als ein starrer, geschlossener Vorderkantenring.

Der Vorderkantenring ist im Unterschallbetrieb hinter den vorderen Segmenten S7 und S8 verstaut, um eine möglichst störungsfreie Umströmung in diesem Betriebsfall zu ermöglichen. Ein Nicht-Verstauen des Vorderkantenrings, vgl. Abbildung 4.43, könnte noch etwas kleinere Segmente S7/8 und kleinere Spalte bei einer annähernd gleichwertigen Geometriegüte ermöglichen. Folglich könnte auch diese Möglichkeit gewählt werden.

Die Verbindung des Vorderkantenrings mit den Segmenten S7 und S8 erfolgt über Gelenkverbindungen, die auf Schienen entlanggleiten. Die Gelenkverbindungen bestehen dabei aus jeweils einem Bolzen BRV7/8, der über ein Verbindungsteil VS7/8 fest mit dem jeweiligen Segment S7/8 verbunden ist. Die Schienen verlaufen hierbei auf einer Bahnkurve, die derart geformt ist, dass Spalte und Überlappungen im Lippenbereich minimiert werden. Trotz der Rundung und des eingeschränkten vorhandenen Bauraums in der Vorderkante des Stellrings sollten zwei Verbindungsstäbe VS7 bzw. VS8 und Gelenkbolzen BRV7 bzw. BRV8 pro $15^{\circ}$-Sektor eingeplant werden. Die Integration eines zweiten Verbindungsstabs pro Sektor erhöht die Sicherheit gegen Segmentverlust, beispielsweise im Vogelschlagfall.

Die Schienen werden intern in den Bauraum des Vorderkantenrings $\mathrm{R}$ integriert, da sie so die Strömung im geringstmöglichen Umfang beeinflussen und im Vergleich zu alternativen Umsetzungen die größte Lebensdauer und Zuverlässigkeit aufweisen. Alternativ könnten die Schienen extern auf dem Vorderkantenring angebracht sein, wo sie die Strömung stärker beeinflussen würden und zudem anfälliger für Verschmutzungen wären, vgl. Abbildung 4.44 Mitte. Weiterhin 


\section{Verstauter Vorderkantenring Freier Vorderkantenring}

Unterschallflug
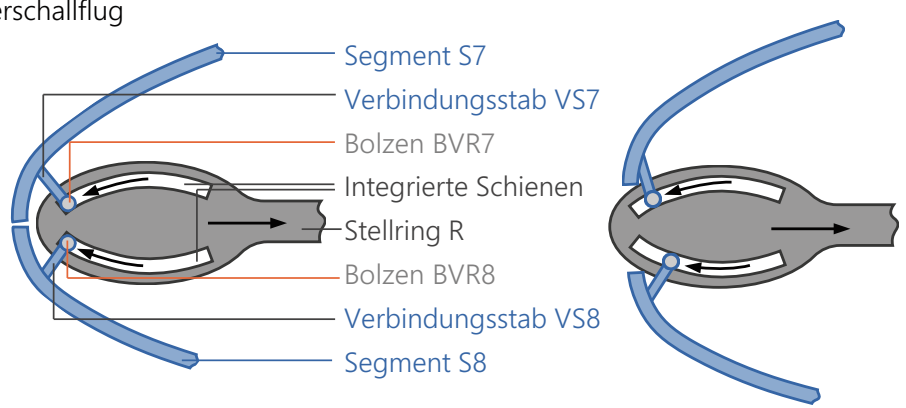

Überschallflug
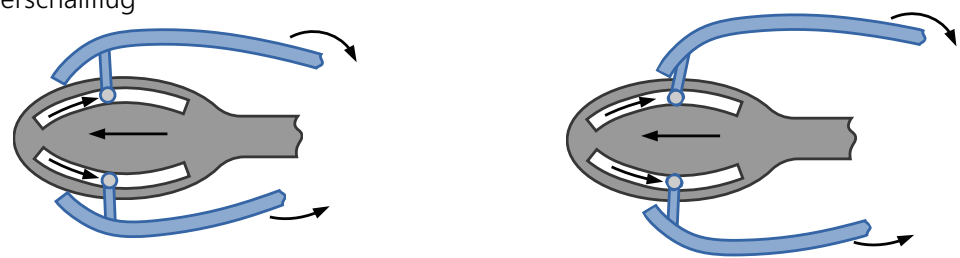

Abbildung 4.43 Optionen der Ausführung des Vorderkantenrings

besteht auch die Möglichkeit, auf Schienen zu verzichten und stattdessen eine Verbindung mit Hilfe eines Elastomers zu realisieren, welches die Segmente S7 und S8 mit dem Vorderkantenring verbindet, vgl. Abbildung 4.44 rechts. Die Lebensdauer dieser Elastomer-Komponente wäre jedoch aufgrund der auftretenden mechanischen und thermalen Beanspruchungen stark eingeschränkt. Weiterhin würde durch die Einspannung eine Krümmung der Geometrie verhindert werden, was wiederum die Einlassumströmung beeinträchtigen kann. Dennoch bietet die Variante einer Elastomerlippe den Vorteil eines geringen erforderlichen Bauraums. Aus diesem Grund findet sie beim Bau eines maßstabsgetreuen verkleinerten Funktionsdemonstrators Anwendung, vgl. Abschnitt 4.5.4.

\section{Umgang mit dem Risiko eines Vogelschlags}

Die durchgeführten Sicherheitsanalysen, insbesondere die PRA, vgl. Abschnitt 4.4.1, stellen die Bedeutung des Vogelschlagrisikos heraus. Im Vogelschlagfall besteht die Gefahr, dass Bauteile des Konzepts stark plastisch verformt werden, strukturell versagen oder auch verloren gehen können. Daraus 
Schienen innen

Schienen außen

Elastomerlippe

Unterschallflug
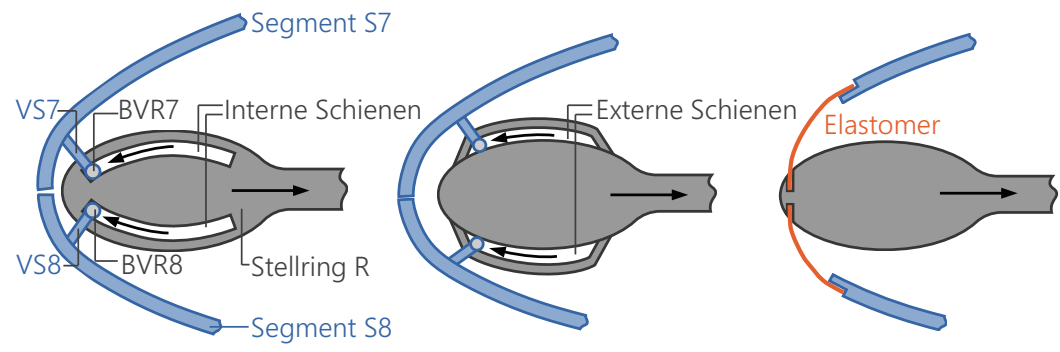

Überschallflug
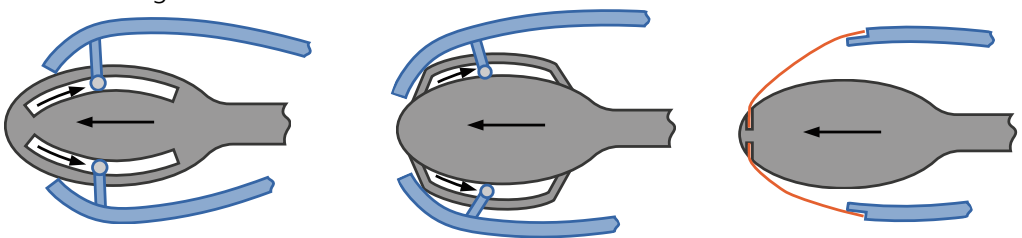

Abbildung 4.44 Optionen der Lippengestaltung

können gefährliche Folgen resultieren. Einerseits können infolge mehrerer von Vogelschlag betroffener Triebwerkseinlässe unzulässige Veränderungen der umströmten Konturen auftreten, die in einem Schubverlust des Flugzeugs resultieren können. Andererseits können verloren gegangene Komponenten sicherheitskritische Bauteile, wie das Leitwerk, beschädigen und somit zum Kontrollverlust über das Flugzeug führen.

Ein Verlust von Konzeptkomponenten kann umgangen werden, indem die Bauteile gegen Vogelschlaglasten ausgelegt werden oder redundante Vorkehrungen [53, S. 144] zum Halten der Segmente integriert werden. Die Stabilität des Konzepts im Vogelschlagfall wird vorrangig durch die Auslegung des umlaufenden Vorderkantenrings gewährleistet. Dieser darf sich unter Vogelschlaglasten zwar verformen, allerdings nicht strukturell versagen. Die umströmten Segmente im Lippenbereich S7 und S8, die mit hoher Wahrscheinlichkeit getroffen werden, haben kleine Abmaße und folglich eine geringe Masse, sodass die Folgen eines potenziellen Abfallens beherrschbar wären. Dies wird im Rahmen der anschließenden ersten Auslegung nachgewiesen, vgl. Anhang A.6.4. 
Zudem sind, wie auch bei allen weiteren umströmten Segmenten, die Verbindungsstäbe und Gelenke redundant ausgeführt. In der nachfolgenden strukturellen Dimensionierung wird gezeigt, ob zudem eine Auslegung dieser Komponenten gegen Vogelschlag möglich ist. Ist besagte Auslegung aufgrund des eingeschränkten Bauraums oder der großen resultierenden Masse nicht möglich, wird ein redundanter Haltemechanismus für die Segmente erforderlich, der sich im Fall des Versagens eines Verbindungsstabs oder Gelenkbolzens aktiviert. Hierfür kann beispielsweise eine im regulären Betrieb unbelastete Stahlseilhalterung verwendet werden, die die benachbarten Segmente miteinander und mit dem Stellring verbindet. Auf diese Weise wäre die Umströmung des Einlasses nach einem Vogelschlag zwar gestört und die Einlassgeometrie könnte nicht mehr variiert werden, aber der gefährliche Verlust eines oder mehrerer Segmente würde vermieden werden.

\subsubsection{Strukturelle Dimensionierung}

Nach der Festlegung des grundlegenden Aufbaus des Einlasskonzepts, wurden seine wichtigsten Komponenten basierend auf den auftretenden Belastungen mittels Festigkeitsnachweis ausgelegt. Dies bedeutet, dass Dimensionen und Materialien der Konzeptkomponenten in der Art gewählt werden, dass die strukturelle Festigkeit der Komponenten beim Wirken der regulär auftretenden Ermüdungsund Grenzlasten, vgl. Abschnitt 4.1.1, gewährleistet ist. Der Festigkeitsnachweis erfolgte für die folgenden Komponenten:

- die umströmten Segmente S1 bis S8,

- den Stellring R,

- die Führungsschienen F,

- die Verbindungsstäbe VS1 bis VS8,

- die Gelenkbolzen BSG1/2, BS13/24/35/46/57/68, BSV1 bis BSV6, BRV1 bis BRV8 sowie

- die Aktorik A.

In Abhängigkeit der vorrangingen Beanspruchung einer Komponente wurde hierbei das Versagen durch Gewaltbruch, unzulässig große Verformungen oder Knicken untersucht.

Für weiterführende Versuche am Boden oder in der Luft sind zusätzliche Auslegungsrechnungen auf einem höheren Detaillierungsgrad erforderlich. Zudem müssen die hier noch nicht detailliert untersuchten Konzeptkomponenten, wie Schrauben und Dichtungen, dimensioniert werden. 


\section{Vorhandene Bauteilbeanspruchungen}

Grundlage eines jeden Festigkeitsnachweises ist die Ermittlung der vorhandenen Bauteilbeanspruchung. Bauteile können mechanisch auf Zug, Druck, Biegung, Schub, Torsion, Flächenpressung, Knicken, Beulen und Kippen beansprucht werden, wobei sich diese Beanspruchungen auch überlagern können [302, S. 43-45], [305], [359]. Die genannten Beanspruchungen können statisch oder in verschiedenen dynamischen Abstufungen auftreten [302, S. 43-45]. Weiterhin können aufgrund variierender Temperaturen auf der Einlassoberfläche thermisch induzierte Spannungen auftreten. Zudem ist bei der Auswahl möglicher ElastomerWerkstoffe für Abdichtungsaufgaben zu beachten, dass durch die Reibung im Überschallbereich hohe Temperaturen erreicht werden können. So traten bei Fluggeschwindigkeiten von Mach 2 auf der Oberfläche der Concorde Temperaturen von über $100{ }^{\circ} \mathrm{C}$ auf [360].

Für die erste Dimensionierung des Einlasskonzepts wurden vorrangig die wirkenden Strömungslasten $F_{\text {Strömung }}$ und $p_{\text {Strömung }}$ sowie die Vogelschlaglast $F_{V o g e l}$ verwendet, vgl. Abbildung 4.45. Die Ermittlung dieser Lasten ist in Anhang A.1 ausführlich beschrieben. Zudem wurden bei der Auslegung die wirkenden Gewichtskräfte $F_{\text {Gewicht }}$ berücksichtigt.

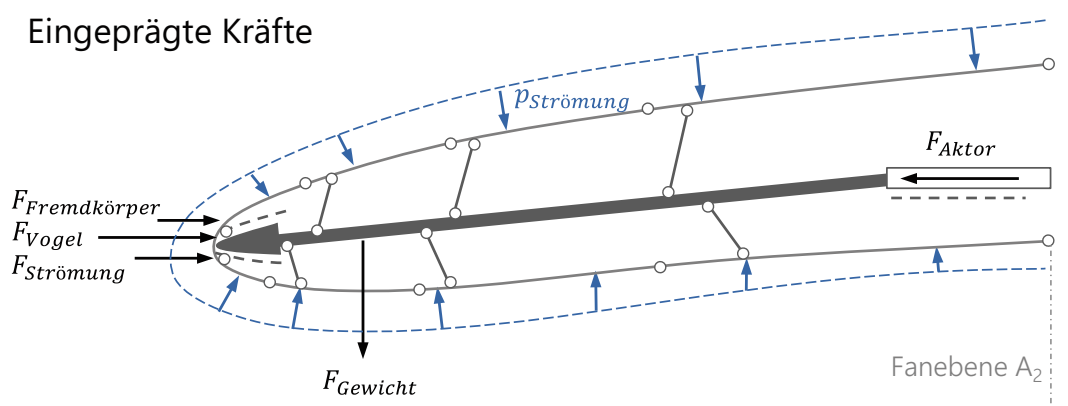

Abbildung 4.45 Einlassbelastungen

Fremdkörpereinschläge $F_{\text {Fremdkörper }}$, zu denen Hagel, Regen und angesaugte Kleinteile, wie Schrauben, Nieten und Steinchen, zählen, wurden im Rahmen dieser Auslegung nicht explizit beachtet, da sich deren Einfluss auf variable Einlässe nicht signifikant von dem auf konventionelle Einlässe unterscheidet.

Zukünftig könnte auch die Auslegung gegen den Einschlag kleinerer Drohnen bis etwa $2 \mathrm{~kg}$ erforderlich werden. Nach aktuellem Stand existieren hierzu 
für den Einlass noch keine relevanten behördlichen Nachweisforderungen. In Abschnitt 4.5.5 werden die Risiken einer Kollision mit einer Drohne erläutert.

Aus Abbildung 4.46 gehen die Belastungen hervor, die auf die freigeschnittenen Komponenten des variablen Systems wirken. Die in orangener Farbe hervorgehobenen Belastungen wurden bei der Auslegung vorrangig berücksichtigt.

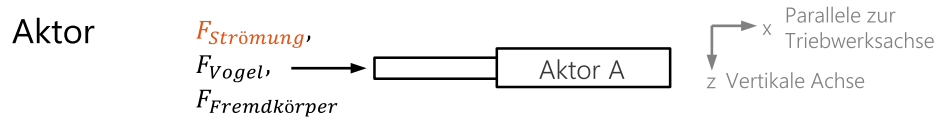

Führungsschienen
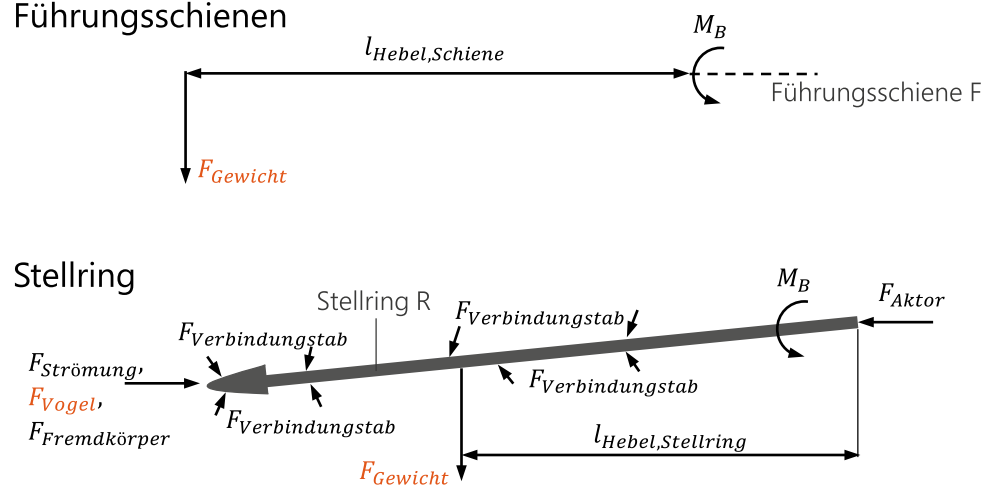

Segmente

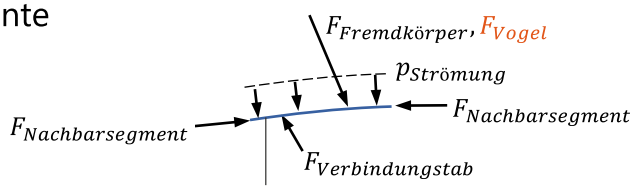

Segment $S$

\section{Verbindungsstäbe}
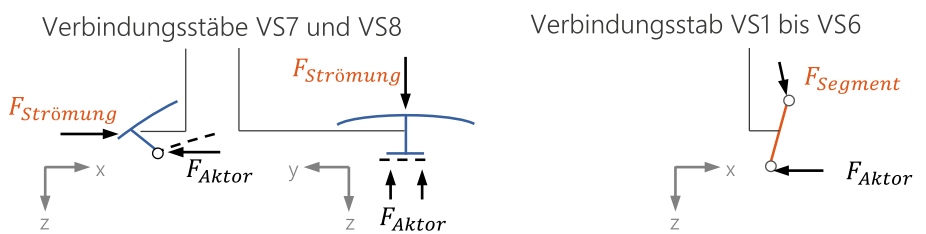

Abbildung 4.46 Freigeschnittene Einlasskomponenten 
Aus den Belastungen, die auf den Einlass und seine Komponenten wirken, ergeben sich die Beanspruchungen auf seine Komponenten und die potenziell daraus resultierenden Versagensformen, wie unzulässig große Verformungen, Knicken, Gewaltbruch, mechanische Abnutzung oder Beulen, vgl. Tabelle 4.19.

Die Auslegung der einzelnen Komponenten gegen die ermittelten Beanspruchungen ist Anhang A.6 zu entnehmen.

Tabelle 4.19 Auf die Einlasskomponenten wirkende Beanspruchungen

\begin{tabular}{|c|c|c|c|}
\hline ID & Bauteilbezeichnung & Vorrangige Beanspruchungen & Auslegung \\
\hline G & Statische Gondel & - & - \\
\hline A & Aktorik & $\begin{array}{l}\text { Zug-/Druckbeanspruchung beim } \\
\text { Stellvorgang und im regulären Flugbetrieb; } \\
\text { Knickbeanspruchung bei Vogelschlag }\end{array}$ & Anhang A.6.6 \\
\hline $\mathrm{F}$ & Führungsschienen & $\begin{array}{l}\text { Biegung durch Gewichtskraft der zu } \\
\text { tragenden Komponenten, Strömungs- und } \\
\text { Manöverlasten }\end{array}$ & Anhang A.6.3 \\
\hline $\mathrm{R}$ & Stellring & $\begin{array}{l}\text { Biegung, vgl. Führungsschienen; } \\
\text { Schub-, Druck- und Knickbeanspruchung bei } \\
\text { Vogelschlag; } \\
\text { Erosion, Hagel und Fremdkörpereinschläge; } \\
\text { thermomechanische Ermüdung und } \\
\text { Spannungen durch Enteisungssystem und } \\
\text { Umgebung }\end{array}$ & Anhang A.6.2 \\
\hline $\mathrm{S} 1$ bis $\mathrm{S} 8$ & Segmente 1 bis 8 & $\begin{array}{l}\text { Druck-/Zugbeanspruchung durch } \\
\text { Strömungslast; } \\
\text { Erosion, Hagel und Fremdkörpereinschläge; } \\
\text { thermomechanische Ermüdung durch } \\
\text { Enteisungssystem und Umgebung; } \\
\text { Schub- und Reibungsbeanspruchung durch } \\
\text { Segmente benachbarter Sektoren }\end{array}$ & Anhang A.6.1 \\
\hline VS1 bis VS8 & $\begin{array}{l}\text { Verbindungsstäbe } 1 \\
\text { bis } 8\end{array}$ & $\begin{array}{l}\text { Druck-, Zug-, Biege- und } \\
\text { Knickbeanspruchung durch Strömungs- und } \\
\text { Aktorlasten sowie Hagel-, Vogel- und } \\
\text { Fremdkörpereinschläge }\end{array}$ & Anhang A.6.4 \\
\hline $\begin{array}{l}\text { BSG1/2, } \\
\text { BS13/24/35/ } \\
46 / 57 / 68, \\
\text { BSV1 bis } \\
\text { BSV6, BRV1 } \\
\text { bis BRV6, }\end{array}$ & Gelenkbolzen & $\begin{array}{l}\text { Biegung, Scherung und Flächenpressung } \\
\text { durch Strömungs- und Aktorlasten sowie } \\
\text { Hagel-, Vogel- und Fremdkörpereinschläge }\end{array}$ & Anhang A.6.5 \\
\hline BRV7/8 & $\begin{array}{l}\text { Gelenkbolzen in } \\
\text { Segment } 7 \text { und } 8\end{array}$ & $\begin{array}{l}\text { Vgl. Gelenkbolzen; } \\
\text { zusätzlich Reibverschleiß beim Stellvorgang }\end{array}$ & Anhang A.6.5 \\
\hline
\end{tabular}




\section{Dimensionen der benötigten Komponenten}

Aus der Auslegung der Systemkomponenten gegen die potenziell wirkenden Beanspruchungen ergeben sich die in Tabelle 4.20 aufgelisteten Bauteildimensionen und gewählten Werkstoffe. Die Masse der jeweiligen Komponenten lässt sich aus dem Produkt ihres ungefähren Volumens und der Dichte des verwendeten Materials bestimmen. Die Volumina der Komponenten berechnen sich aus den gewählten Bauteilabmessungen, deren Auslegung in Anhang A.6 detailliert beschrieben ist. Für die Aluminiumknetlegierungen EN AW-2024 und EN AW $7075 \mathrm{kann}$ eine Dichte von $2,7 \mathrm{~g} / \mathrm{cm}^{3}$ angenommen werden und für Stahl S275JR eine Dichte von $7,85 \mathrm{~g} / \mathrm{cm}^{3}$. Somit ergibt sich eine Gesamtmasse der dimensionierten Komponenten des Einlasskonzepts von etwa $172 \mathrm{~kg}$.

\subsubsection{Rechnerunterstützte 3D-Modellierung und Gestaltung}

Basierend auf den erforderlichen Bauteilabmessungen, die aus den Untersuchungen zur Kinematik sowie der ersten Konzeptauslegung hervorgehen, erfolgte die 3D-Modellierung des Konzepts mit dem CAD-System Siemens NX12. Nachfolgend werden die einzelnen 3D-modellierten Komponenten und deren mechanische Verbindungen miteinander vorgestellt.

\section{Statische Gondel}

Von der statischen Gondel $G$ wurde nur der starre Bereich des Einlasses als ein zusammenhängendes Bauteil modelliert. Dieser überträgt durch eine Flanschverbindung sämtliche auf das variable Konzept wirkenden Belastungen auf die restliche Triebwerksgondel. Der umströmte Anteil des starren Bereichs beträgt $30 \mathrm{~mm}$. Dies stellt sicher, dass direkt vor dem Fan eine ideale Kreisquerschnittsfläche des Einlasses vorliegt. Beim Übergang zwischen dem statischen und dem variablen Bereich sind Stufen und Spalte zu minimieren, um Störungen der Strömung zu vermeiden, vgl. Abbildung 4.41.

Weiterhin sind zur Übertragung der wirkenden Belastungen Montageflächen für die Führungsschienen F und die Aktorik A vorgesehen, vgl. Abbildung 4.47.

Für die Funktion des variablen Einlasses sind Kabel und Leitungen erforderlich, um beispielsweise Strom für die Aktorik oder das Enteisungssystem bereitzustellen. Diese müssen aus der restlichen Gondel durch den Verbindungsflansch zu den Komponenten des variablen Systems geleitet werden. Der Bauraum im variablen Einlass ist ausreichend für die Unterbringung dieser Leitungen. 


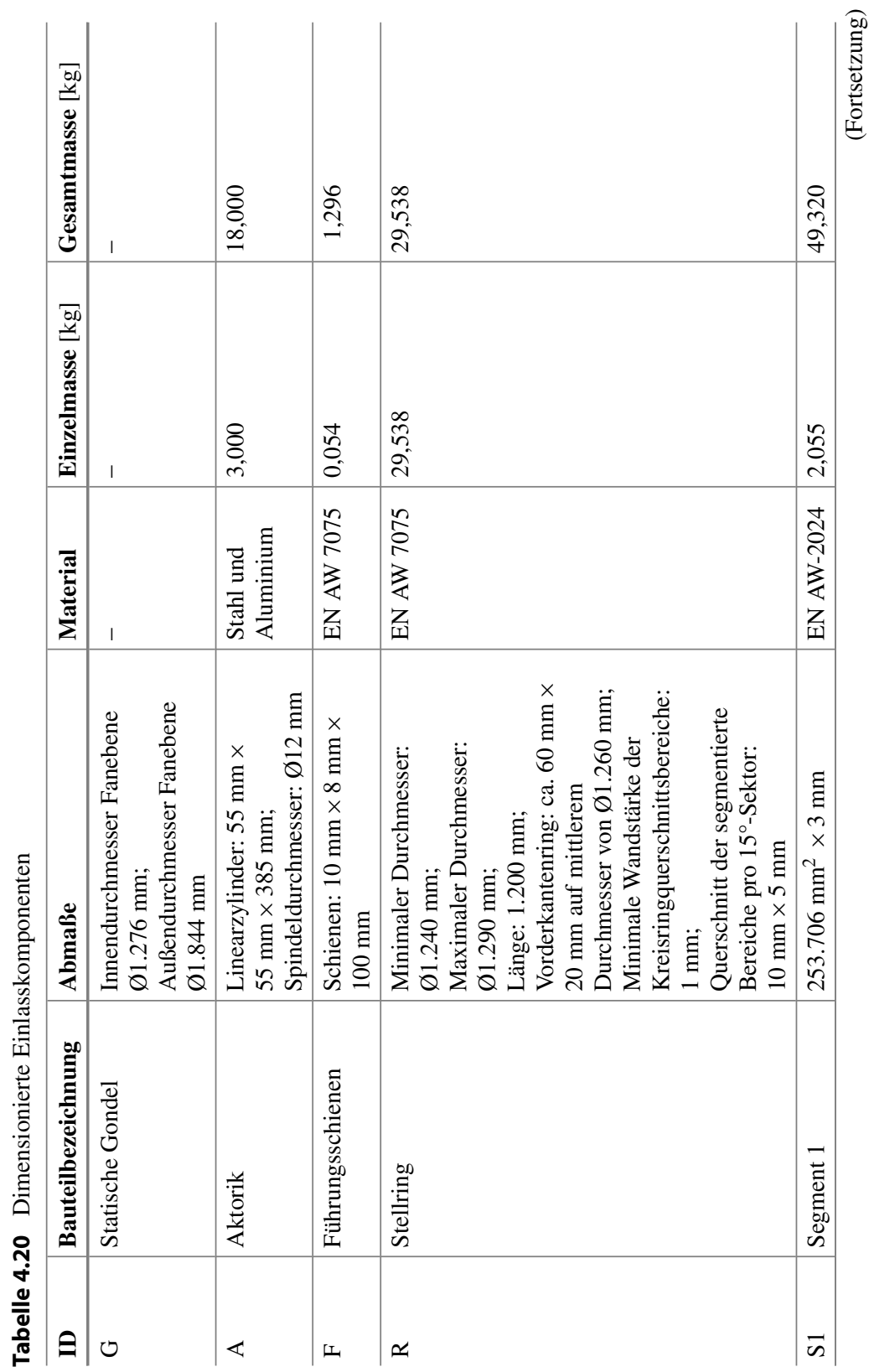




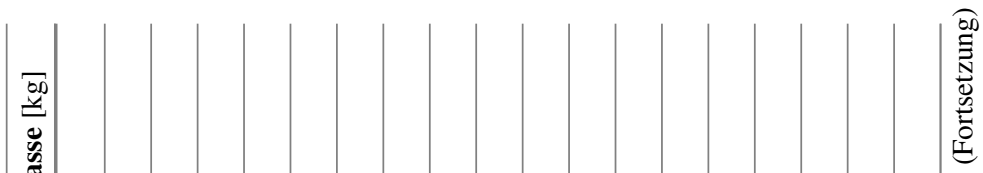

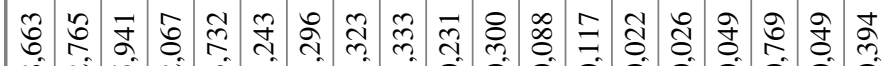

चु

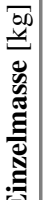

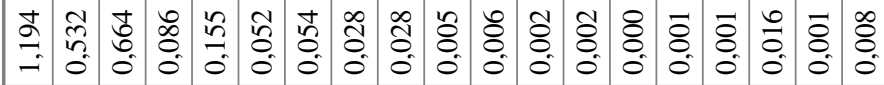

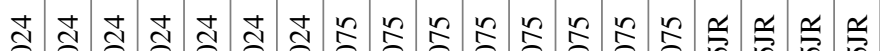

ส

焉

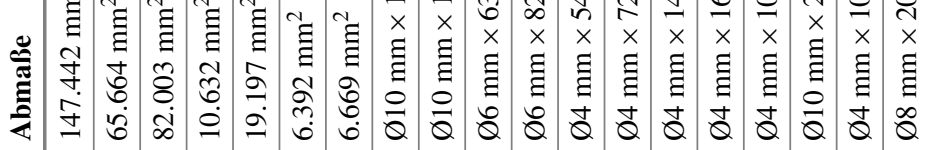

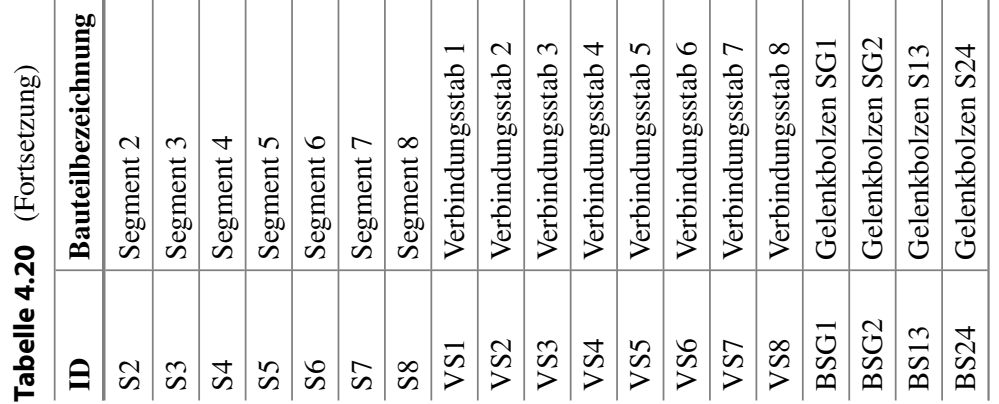




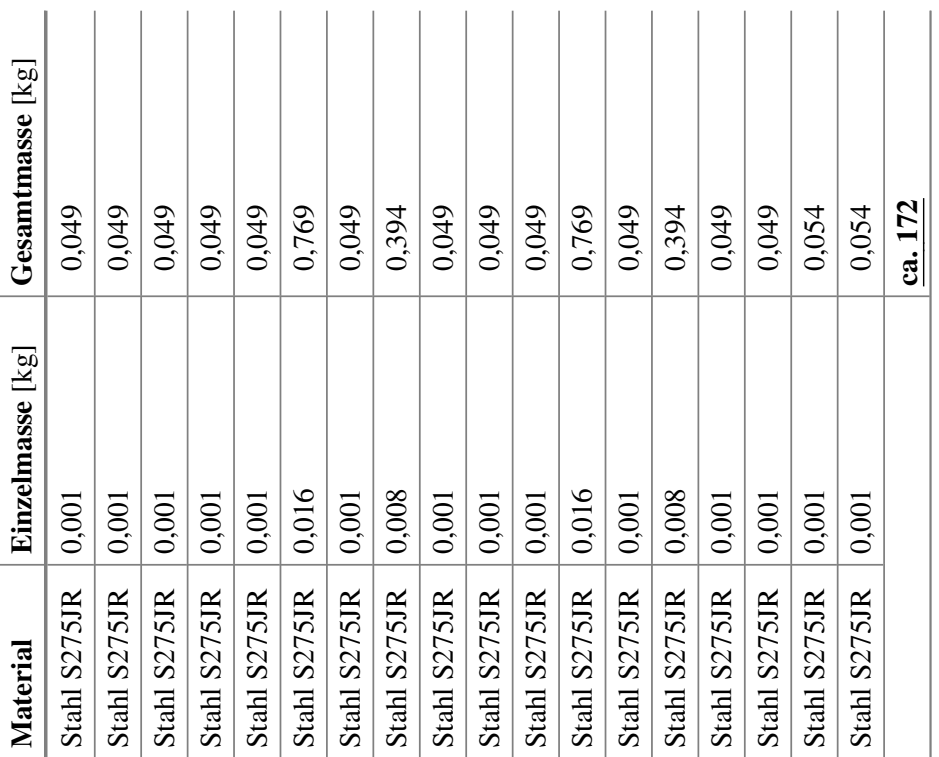

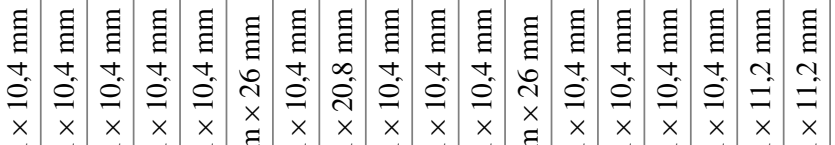

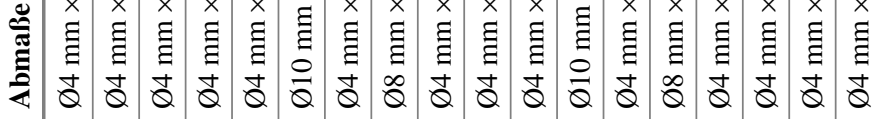

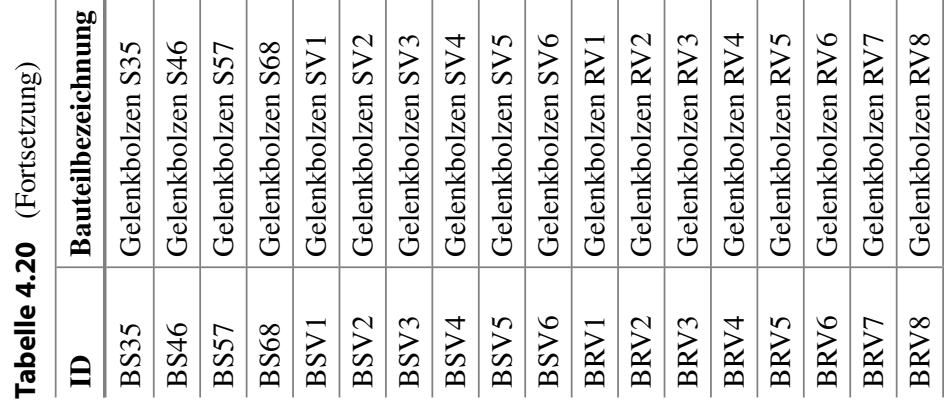




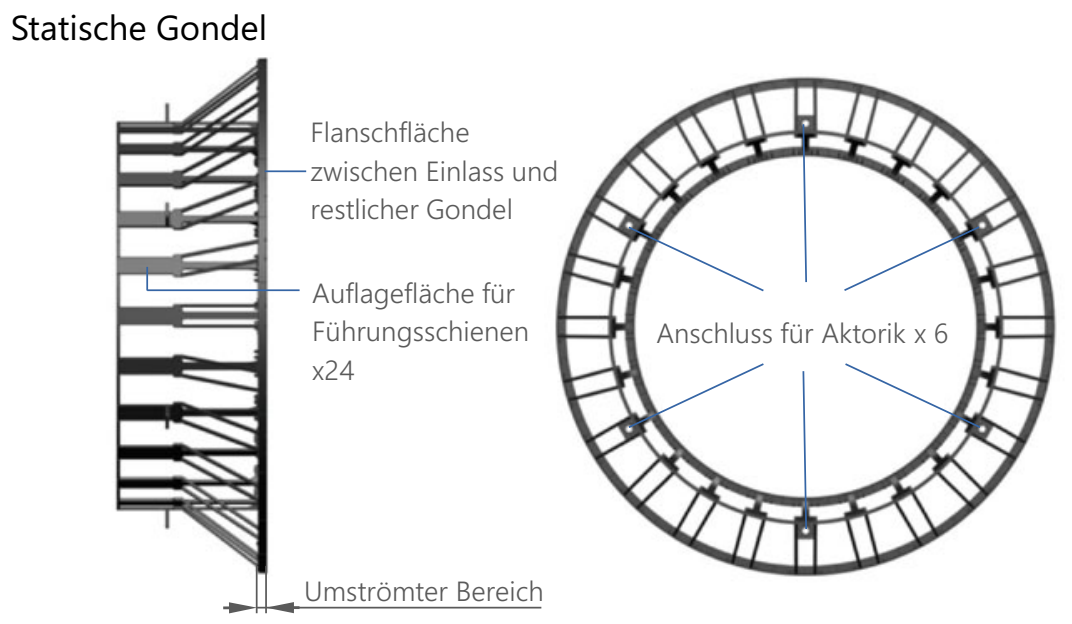

Abbildung 4.47 Statischer Teil des Einlasses

Bei der Installation dieser Kabel und Leitungen sind jedoch auch Interaktionen mit den variablen Komponenten zu vermeiden, vgl. Abschnitt 4.4.1. Aus diesem Grund sollten in einer späteren Entwicklungsphase die Kabelwege modelliert und detailliert untersucht werden.

\section{Aktorik}

Die erforderlichen Komponenten der Aktorik A bestehen aus Stelltrieb, Motorsteuerung und Leitungen. Diese Komponenten wurden gemäß ihrer erforderlichen Abmessungen, vgl. Anhang A.6.6, vom Hersteller Festo übernommen [361]. CAD-Modelle des ausgewählten Stelltriebs und der zugehörigen Motorsteuerung sind in Abbildung 4.48 dargestellt [361]. Als Stelltriebe werden sechs Elektrozylinder der Baureihe EPCO-40-50-5P-ST-E [361] verwendet. Diese werden im statischen Teil des Einlasses montiert und die ausfahrbaren Stellglieder mit dem Stellring R verbunden. Die Motorsteuerung CMMO-ST-C5-1-DIOP könnte auch innerhalb des statischen Bereichs des Einlasses installiert werden. Sie erhält ein Stellsignal von der triebwerksseitigen Aktorsteuerung und beliefert die Elektrozylinder mit der erforderlichen Stellenergie, vgl. Abschnitt 2.4.3. 

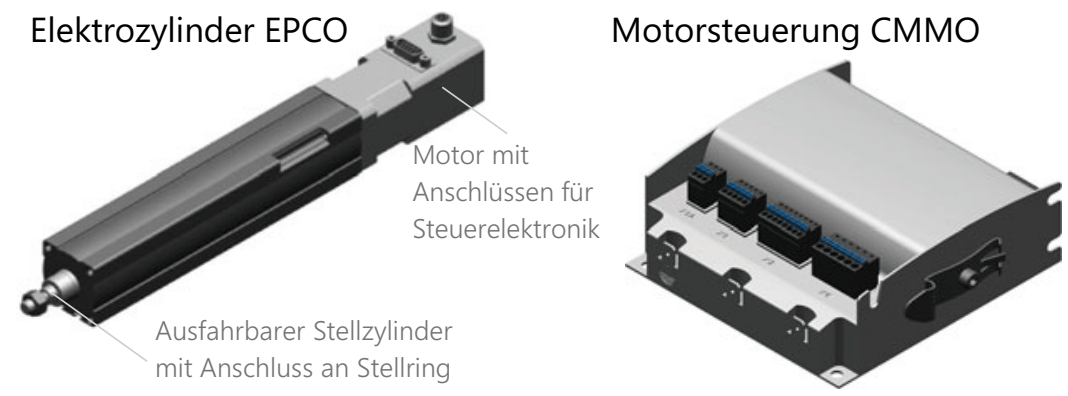

Abbildung 4.48 Aktorik des variablen Einlasskonzepts

\section{Führungsschienen}

Die Führungsschienen F sind als Gleitschienen bestehend aus Schiene und Schlitten ausgeführt, vgl. Abbildung 4.49. Die Schiene ist hierbei fest mit der Auflagefläche im statischen Teil des Einlasses verbunden, vgl. Abbildung 4.47. Der Schlitten ist fest mit dem Stellring $\mathrm{R}$ verbunden und stellt somit dessen axiale Führung sicher. Für die festen Verbindungen der Bauteile untereinander können beispielsweise Schraubverbindungen verwendet werden. Sowohl die Profilflächen der jeweiligen Schienen als auch die der Schlitten erreichen die strukturell erforderliche Querschnittsfläche von $80 \mathrm{~mm}^{2}$, um den wirkenden Biegebeanspruchungen standzuhalten, vgl. Anhang A.6.3.

\section{Führungsschienen}
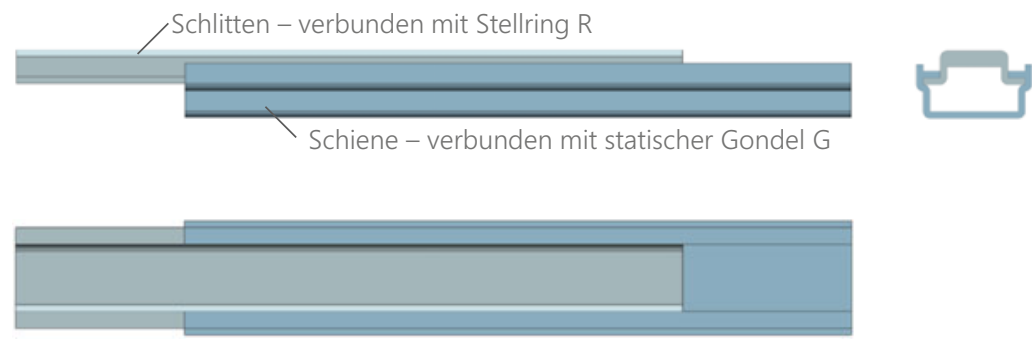

Abbildung 4.49 Führungsschienen 


\section{Stellring}

Der Stellring $\mathrm{R}$ wird über die Führungsschienen $\mathrm{F}$ mit dem statischen Teil des Einlasses $\mathrm{G}$ verbunden und gelagert. Zudem wird seine axiale Position mittels der sechs über den Umfang verteilten Aktoren A gesteuert. Aus Gründen der Massenreduktion wird der Stellring nicht durchgängig als Ring, sondern größtenteils segmentiert ausgeführt, vgl. Abbildung 4.50.
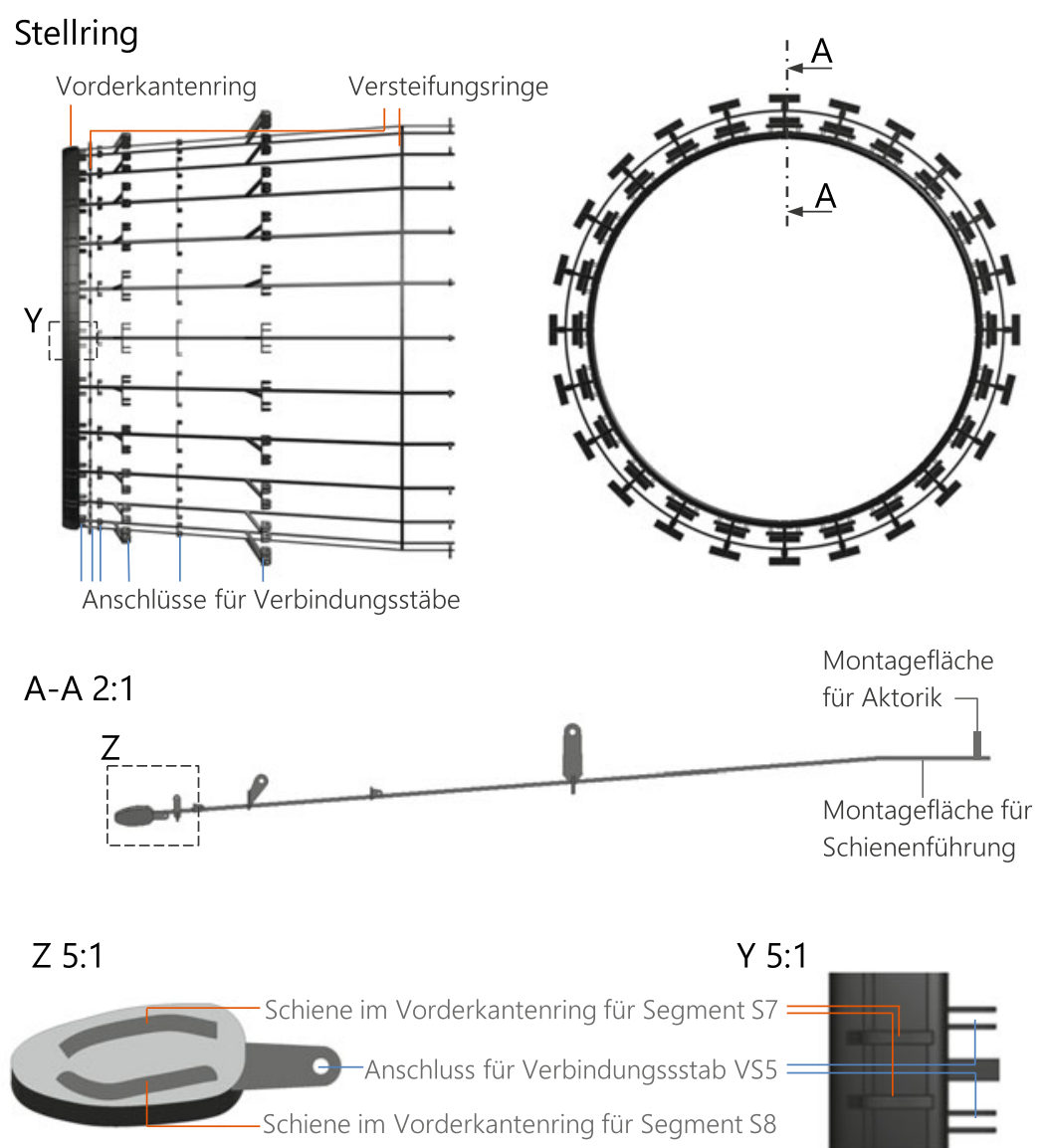

Abbildung 4.50 Stellring 
An den Aufnahmestellen der Verbindungsstäbe ist er jedoch zur Versteifung der Konstruktion ringförmig gestaltet. Auch im Bereich der Vorderkante ist der Stellring als durchgängiger Ring realisiert. Zudem sind in diesem Bereich die Schienen zur Aufnahme der Gelenkbolzen BRV7/8 der Segmente S7/8 integriert, vgl. Detail Z in Abbildung 4.50.

\section{Verbindungsstab}

Die Verbindungsstäbe VS1 bis VS6 sind gemäß ihrer Auslegung in Anhang A.6.4 dimensioniert. Dies ist in Abbildung 4.51 am Beispiel von Verbindungsstab VS5 dargestellt.

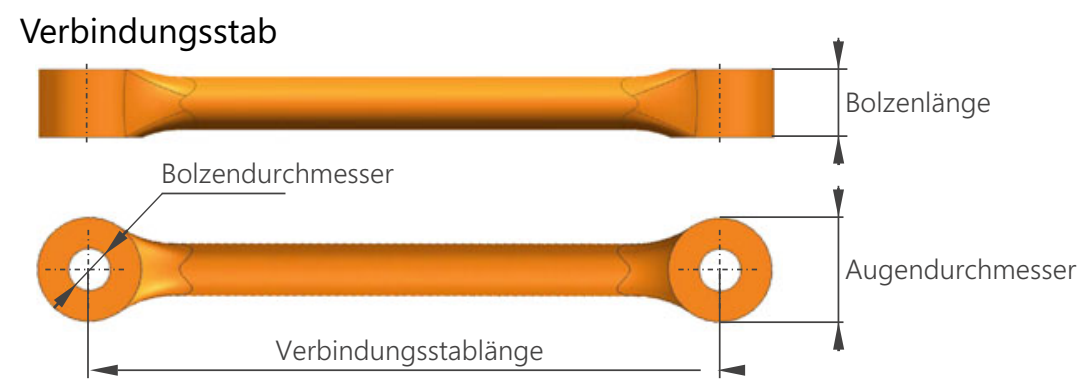

Abbildung 4.51 Verbindungsstab VS5

\section{Gelenkverbindungen zwischen Stellring und Verbindungsstäben}

Mit Hilfe der in Anhang A.6.5 dimensionierten Bolzen werden die Verbindungsstäbe VS1 bis VS6 am Stellring montiert, vgl. Abbildung 4.52. Dabei ist in der Gabel auf der Seite des Stellrings eine Übermaßpassung realisiert, die einen Verlust der Bolzen durch Herausrutschen verhindert. Zusätzlich kann der Bolzen durch einen Splint gegen axiales Verrutschen gesichert werden. Zwischen den Bolzen und den Verbindungsstäben werden Spielpassungen gewählt, um die Drehbewegung der Verbindungsstäbe zu ermöglichen. 


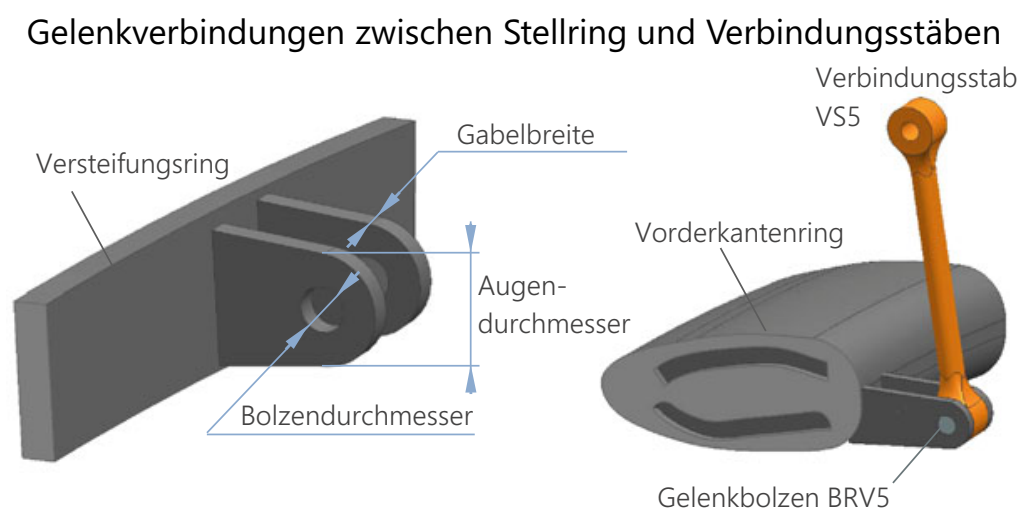

Abbildung 4.52 Gelenkverbindung zwischen Stellring und Verbindungsstab

\section{Segmente}

Die umströmten Segmente S1 bis S8 des Systems basieren auf einem $15^{\circ}$ großen Ringsegment als Grundkörper, vgl. Abbildung 4.53. Zusätzlich sind die Gelenkgabeln und Stangen für die Verbindungen mit dem zugehörigen Verbindungsstab und den Nachbarsegmenten des betreffenden Sektors Bestandteil der Segmente. Dabei ist bei den Segmenten S1 bis S6 für die Verbindung mit dem Vordersegment stets die Gelenkgabel am Segment untergebracht und für die Verbindung mit dem stromabwärtsliegenden Segment die Gelenkstange. Bei den Lippensegmenten S7 und S8 sind die zugehörigen Verbindungsstäbe VS7/VS8 sowie die Bolzen BRV7/BRV8, die im Vorderkantenring entlanggleiten, feste Bestandteile dieser Segmente. Trotz der Rundung und des eingeschränkten vorhandenen Bauraums in der Vorderkante des Stellrings ist es möglich, jeweils zwei Verbindungsstäbe VS7/VS8 und Gelenkbolzen BRV7/BRV8 pro $15^{\circ}$-Sektor zu integrieren.

Daraus resultieren verschiedene Vorteile. Beispielsweise wird das gleichmäßige Abgleiten der Bolzen entlang der Schienen des Stellrings erleichtert. Weiterhin trägt die doppelte Ausführung der Verbindungsstäbe und der Gelenkbolzen zu einer erhöhten Sicherheit gegen Segmentverlust bei, vgl. Anhang A.6. 


\section{Segmente S1 bis S6}

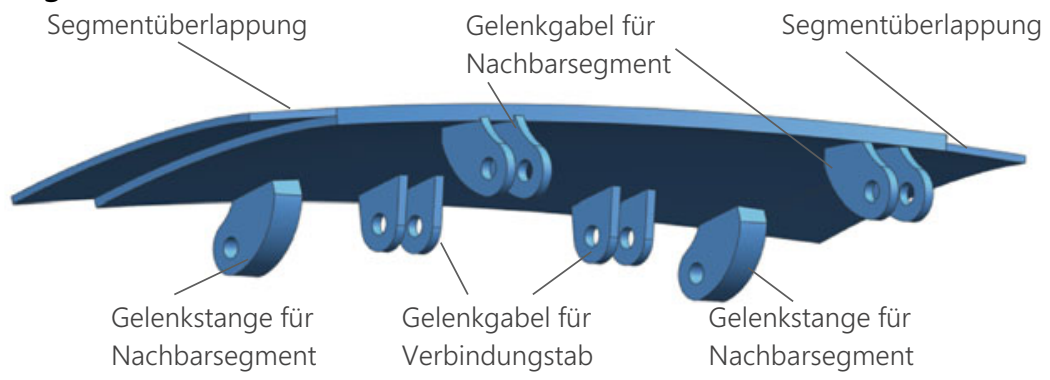

\section{Segmente S7/S8}

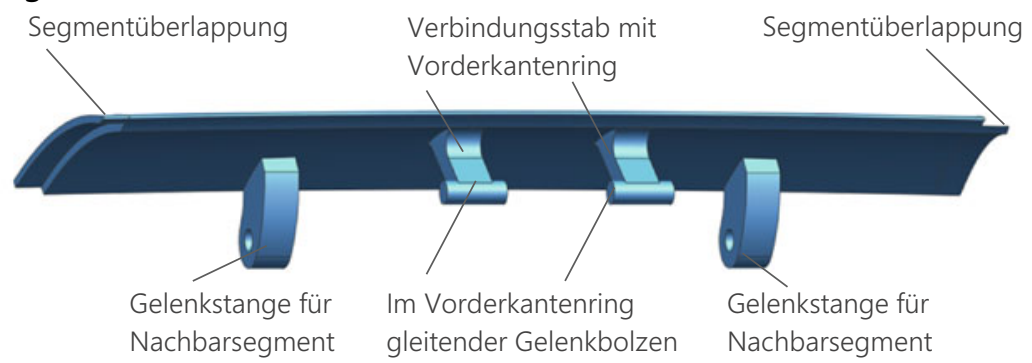

Abbildung 4.53 Aufbau der umströmten Segmente

Wie zuvor beschrieben, werden für die Umsetzung einer Querschnittsvariation Segmentüberlappungen verwendet, vgl. Abbildung 4.11. Diese Überlappungen sind Bestandteil der Segmente und beidseitig in diese integriert. Um bei der Querschnittsvariation das Auftreten größerer Spalte oder von Bauteilkollisionen zu verhindern, müssen die Überlappungen zwischen den Segmenten benachbarter Sektoren parallel zueinander sein, vgl. Abbildung 4.54. Die Erstellung der parallelen Überlappungen wird nachfolgend beschrieben. 


\section{Segmentüberlappungen}

Erstellung eines $15^{\circ}$-Grundkörpers durch Rotation
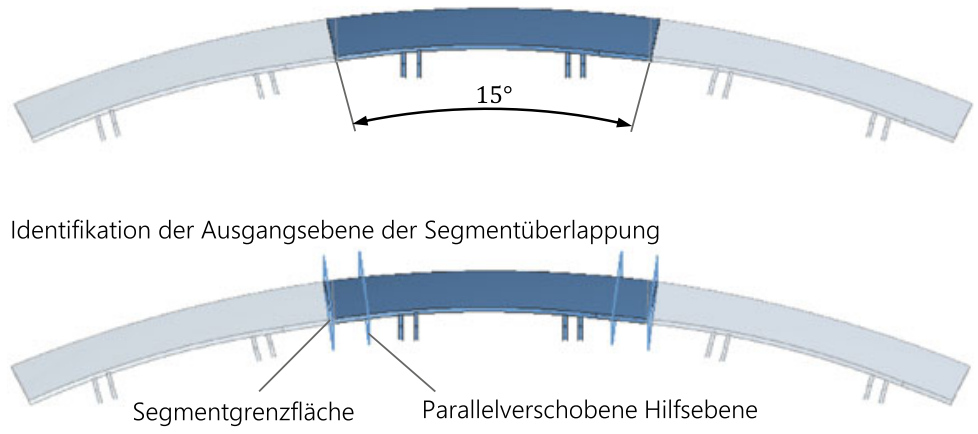

Entfernen des überschüssigen Materials
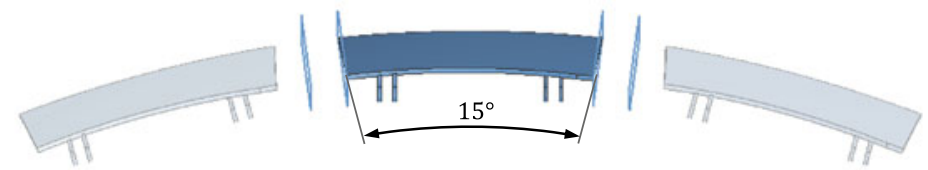

Erstellung linearer Verlängerungen senkrecht zu den Schnittflächen

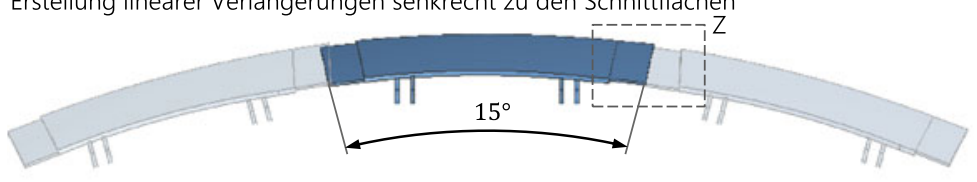

Z 5:1:

Größerer Querschnitt

Kleinerer Querschnitt
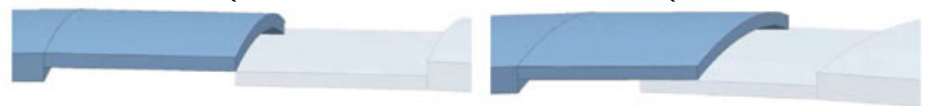

Abbildung 4.54 Gestaltung der Segmentüberlappungen

Die Außenflächen des rotierten Segmentgrundkörpers stehen in einem Winkel von $15^{\circ}$ zueinander. Bei einer Querschnittsvariation würden die Segmentverlängerungen benachbarter Sektoren jedoch miteinander kollidieren. Deshalb werden Hilfsebenen erstellt, die parallel zu den Außenflächen des Grundkörpers liegen. Der Abstand der Hilfsebenen zu den ursprünglichen Außenflächen entspricht dabei der späteren Größe der Segmentüberlappungen. Das Material zwischen den 
erstellten Ebenen und den ursprünglichen Außenflächen wird entfernt. Die somit neu entstehenden Außenflächen weisen ebenfalls einen Winkel von $15^{\circ}$ zueinander auf. Die neuen Außenflächen werden mit der halben Segmentdicke senkrecht zur Schnittfläche linear verlängert. Somit sind die Segmentüberlappungen parallel zueinander.

Dennoch ist die Entstehung kleinerer Spalte im Lippenbereich bei der Querschnittsvariation aufgrund der relativen Drehung der Segmente in der Ebene nicht vermeidbar. Durch die geringe Größe der Sektoren von $15^{\circ}$ werden diese Spalte jedoch minimiert. Aufgrund der Segmentüberlappungen entstehen auf der umströmten Kontur des Einlasses axiale Stufen in Höhe von 1,5 mm.

\section{Gelenkverbindungen zwischen Segmenten}

Wie bei der gelenkigen Verbindung zwischen Stellring $\mathrm{R}$ und den Verbindungsstäben VS1 bis VS6 wird die Verbindung benachbarter Segmente durch Bolzengelenke realisiert, vgl. Abbildung 4.55. Hierbei wird zwischen der Gelenkgabel und dem Bolzen stets eine Übermaßpassung realisiert, um einen Verlust des Gelenkbolzens zu verhindern. Zwischen der Gelenkstange und dem Bolzen wird eine Spielpassung verwendet, um die Drehbewegung der Segmente zueinander zu ermöglichen.

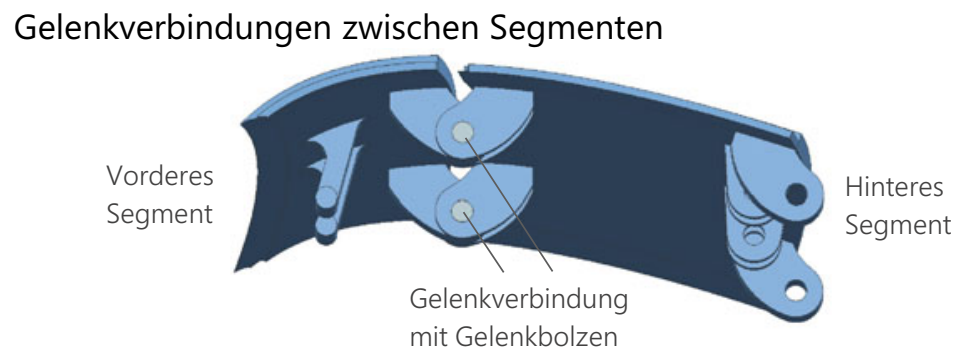

Abbildung 4.55 Gelenkverbindungen zwischen Segmenten

Durch die Rotation der Segmente könnten diese miteinander kollidieren, was durch Aussparungen umgangen werden kann, vgl. Abbildung 4.40. In Abbildung 4.56 sind die real auftretenden Spalte mit einer Größe von bis zu $5 \mathrm{~mm}$ dargestellt. Diese sollten in der weiteren Entwicklung untersucht werden und ggf. durch Elastomere abgedichtet werden, vgl. Abbildung 4.41. 


\section{Aussparungen}

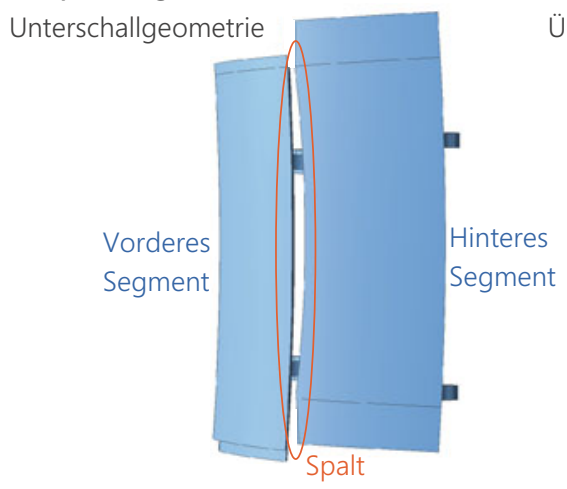

Überschallgeometrie

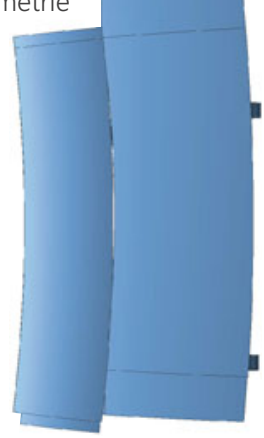

Abbildung 4.56 Erforderliche Aussparungen zwischen Segmenten

\section{Gelenkverbindungen zwischen Segmenten und Verbindungsstäben}

Für die Gelenkverbindungen zwischen den Verbindungstäben VS1 bis VS6 und den zugehörigen Segmenten werden ebenfalls Bolzen verwendet, vgl. Abbildung 4.57. Die Bolzen werden dabei in die segmentseitigen Gelenkgabeln mit Übermaßpassungen und in die Gelenkstangen seitens der Verbindungsstäbe mittels Spielpassungen eingefügt.

Gelenkverbindungen zwischen Segmenten und Verbindungsstäben

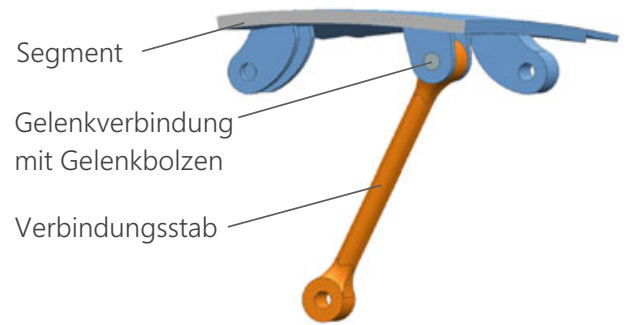

Abbildung 4.57 Gelenkverbindung zwischen Segment und Verbindungsstab 


\section{Gelenkverbindungen zwischen Segmenten und Vorderkantenring}

Die Gelenkbolzen der Segmente S7 und S8 sind über eine Spielpassung in die Schienen des Vorderkantenrings integriert, vgl. Abbildung 4.58.

Gelenkverbindungen zwischen Segmenten und Vorderkantenring

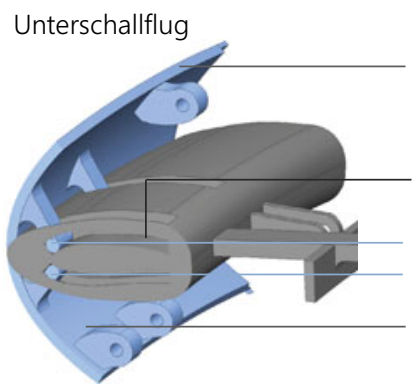

A-A 2:1
Überschallflug

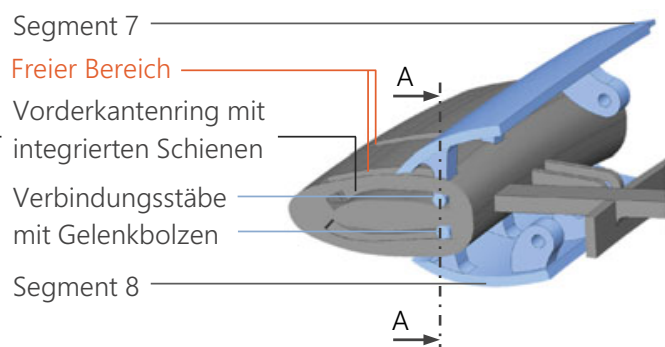

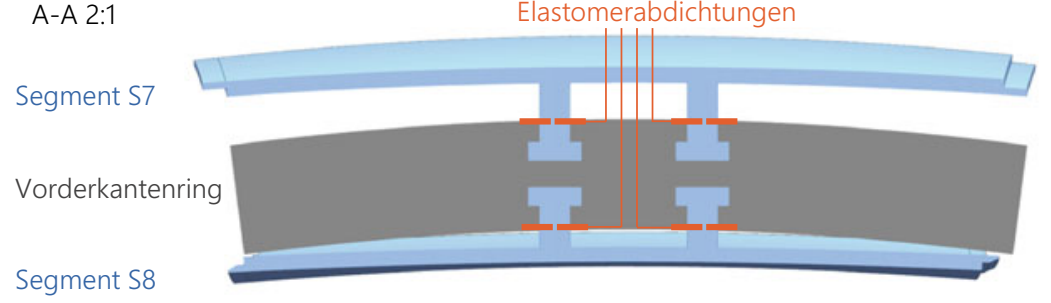

Abbildung 4.58 Gelenkverbindungen zwischen Segmenten und Vorderkantenring

Dies ermöglicht ein Gleiten der Segmente entlang der Schienen und somit eine Variation der Lippengeometrie. Für die Montage muss ein kleiner Zugang zu den Schienennuten in den Vorderkantenring integriert werden. Damit die Bolzen im Betrieb nicht aus dem Vorderkantenring herausfallen können, muss der Zugang nach der Montage wieder verschlossen werden, beispielsweise durch eine verschraubte Abdeckung. 
Beim Verfahren in die Überschallgeometrie kann innerhalb der Schienenkanäle im Vorderkantenring $\mathrm{R}$ ein freier Bereich zwischen den Schienen und den Verbindungsstäben VS7/8 entstehen, vgl. Abbildung 4.58 oben und Detail Y in Abbildung 4.59. In diesen Bereich könnten Schmutz und Wasser eindringen und somit die Funktionsweise des variablen Einlasses gefährden. Dies wäre durch eine Abdichtung aus Elastomeren, die die Schienenkanäle im Vorderkantenring abdichtet, vermeidbar, vgl. Schnitt A-A in Abbildung 4.58.

\section{Vorderansicht Einlasskonzept}

Unterschallflug

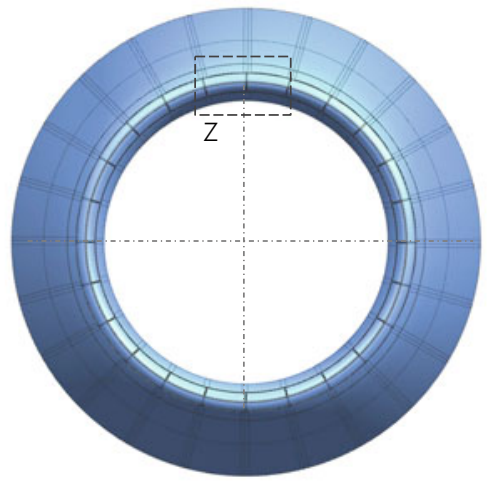

Z 5:1 Spaltentstehung

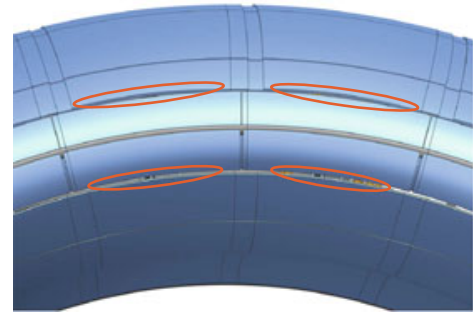

Überschallflug

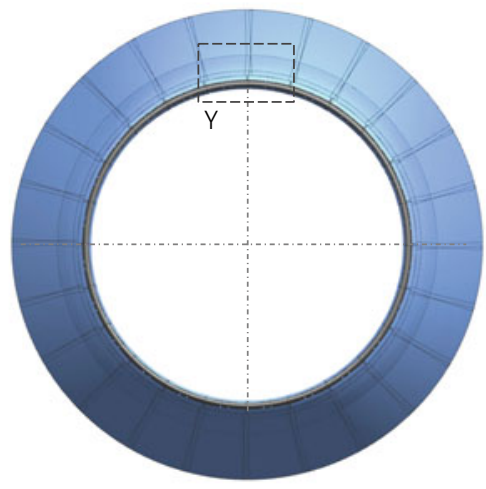

Y 5:1 Verbindungsstäbe VS7/8

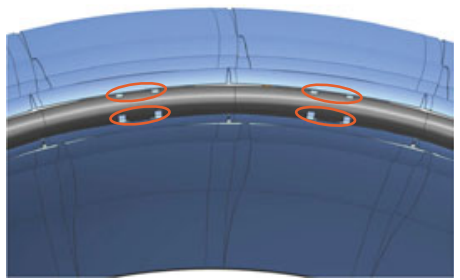

Abbildung 4.59 Vorderansicht des zusammengebauten CAD-Modells 


\section{Zusammenbau}

Die zu einem Gesamtmodell zusammengefügten Komponenten des Konzepts sind in Abbildung 4.59 in der Vorderansicht und in Abbildung 4.60 in der Seitenansicht dargestellt.

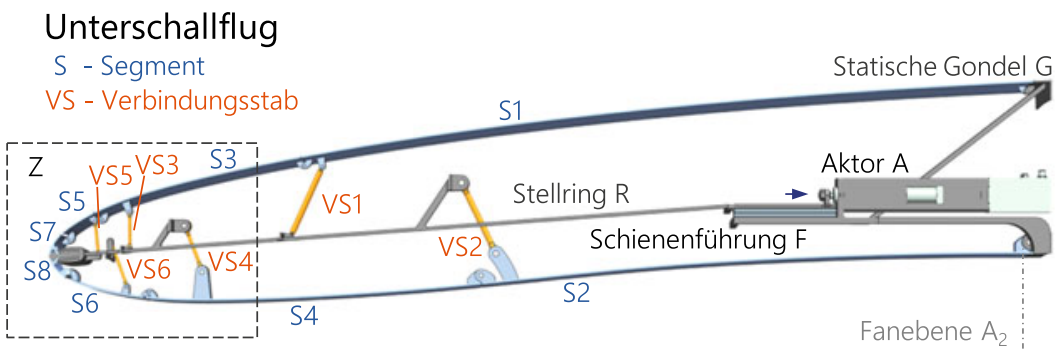

Überschallflug

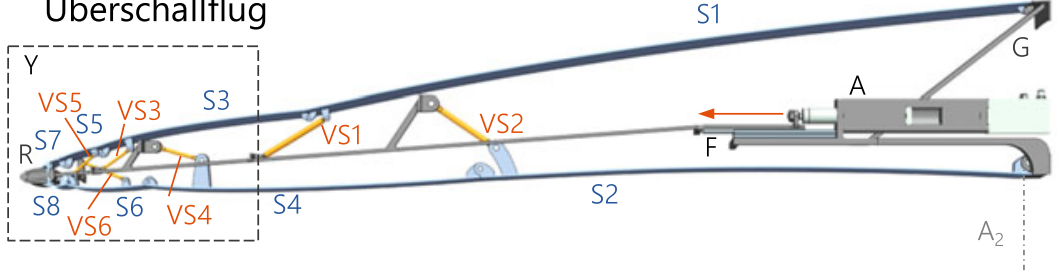

Z 2:1

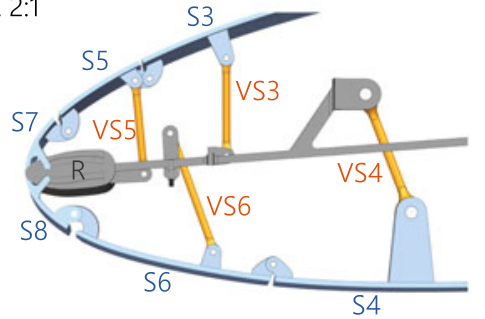

Y 2:1

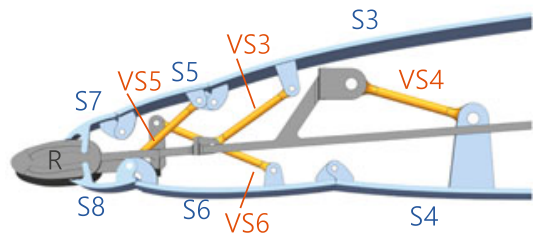

Abbildung 4.60 Schnittansicht eines Sektors des Konzepts

Aus der Bewegungssimulation und Kollisionsprüfung des zusammengebauten CAD-Modells in Siemens NX12 geht hervor, dass keine Komponenten miteinander kollidieren. Dadurch wird die mechanische Funktionsweise des variablen Einlasskonzepts theoretisch nachgewiesen. 
Die Segmentaussparungen zur Vermeidung von Bauteilkollisionen, vgl. Abbildung 4.56, verursachen im Unterschallbetrieb kleine Spalte im Lippenbereich, vgl. Abbildung 4.59 Detail Z. Diese Spalte könnten durch eine optimale axiale Segmentierung der Sektoren deutlich verkleinert werden.

Abbildung 4.60 stellt die geschnittene Seitenansicht eines Sektors dar und erlaubt somit den Vergleich des 3D-modellierten Konzepts mit dem Funktionsprinzip aus Abbildung 4.34. Dabei wird die lippenlastige, axiale Unterteilung der Modellkontur in Segmente deutlich, wodurch eine größere Glattheit der Kontur im Überschallbetrieb ermöglicht wird.

\subsubsection{Funktionsdemonstratoren}

Im Verlauf der Konzeptstudie wurden zwei Funktionsdemonstratoren variabler Einlässe im Maßstab 1:3 gefertigt. Diese Prototypen sind der Konzeptgruppe verschiebbarer starrer Segmente zuzuordnen. Mit Hilfe der Prototypen wurde die Umsetzbarkeit der erarbeiteten Lösungsprinzipe für die Konturvariation aus Abschnitt 4.2.4 nachgewiesen. Dabei wurden erste Erfahrungen für die Fertigung und Montage gesammelt. Im Stellmechanismus finden hierbei jeweils Elektrozylinder mit Kugelgewindetrieb von Festo Anwendung, vgl. Anhang A.6.6. Die Führungsschienen, Scharniere und Schrauben entsprechen handelsüblichen Metallbauteilen. Die übrigen Komponenten entstammen der Fertigung mittels 3D-Druck aus thermoplastischem Kunststoff. Zudem wurden potenzielle Schwachstellen frühzeitig identifiziert und behoben. Ein Nachweis der strukturellen und aerodynamischen Funktionalität sollte Gegenstand anschließender Untersuchungen sein.

\section{Erster Funktionsdemonstrator}

Der Bau des ersten Funktionsdemonstrators wurde im Anschluss an die Konzeptvorauswahl begonnen, vgl. Abschnitt 4.3.2. Dabei wurde das in Abbildung 4.12 dargestellte Konzept umgesetzt, welches die Innen- und Außenkontur des Einlasses durch axiale Bewegung eines Stellglieds variiert. Der gefertigte Prototyp verdeutlicht die Funktionalität des Prinzips des Verschiebens starrer Komponenten für die Variation der Einlassgeometrie, vgl. Abbildung 4.61. 
Abbildung 4.61 Erster

Funktionsdemonstrator

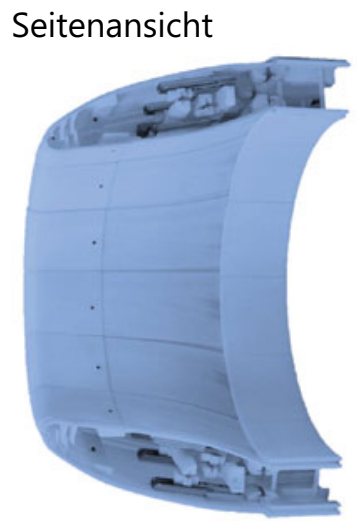

Vorderansicht

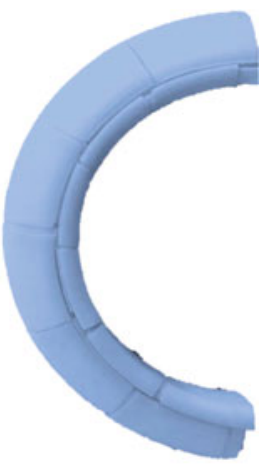

Eine Schwachstelle des verwendeten Konzepts stellt der Bereich der Einlassvorderkante dar. In diesem Bereich können Spalte und Lücken auftreten, die bei Versagen der Elastomerabdichtungen, vgl. Abbildung 4.12, verstärkt werden können. Dadurch könnte die langfristige Funktionsfähigkeit des Einlasssystems gefährdet werden. Zudem ist die stark segmentierte Vorderkante bei Vogelschlägen strukturell anfällig. Deshalb wurde für den zweiten Prototypen ein starrer Vorderkantenring gewählt, vgl. Abschnitt 4.5.1. Zusammenfassend könnte diese Konzeptidee jedoch als Alternative weiterverfolgt werden, falls sich die ausgewählte Variante im weiteren Entwicklungsverlauf aufgrund der höheren Komplexität als unzureichend herausstellen sollte.

\section{Zweiter Funktionsdemonstrator}

Die Umsetzbarkeit des im vorherigen Kapitel hergeleiteten und dimensionierten Konzepts wurde mit Hilfe des zweiten Funktionsdemonstrators praktisch gezeigt, vgl. Abbildung 4.62. Dadurch hat das Konzept den Reifegrad TRL 3 erreicht. Aufgrund des Verkleinerungsmaßstabes wurden die Segmente S7 und S8 im Prototyp durch ein dehnbares Elastomer ersetzt, vgl. Abbildung 4.44 rechts. Die Ausführung unter Verwendung starrer Segmente S7 und S8 ist in Anhang A.7 mittels technischer Baugruppenzeichnungen im Originalmaßstab dargestellt. 

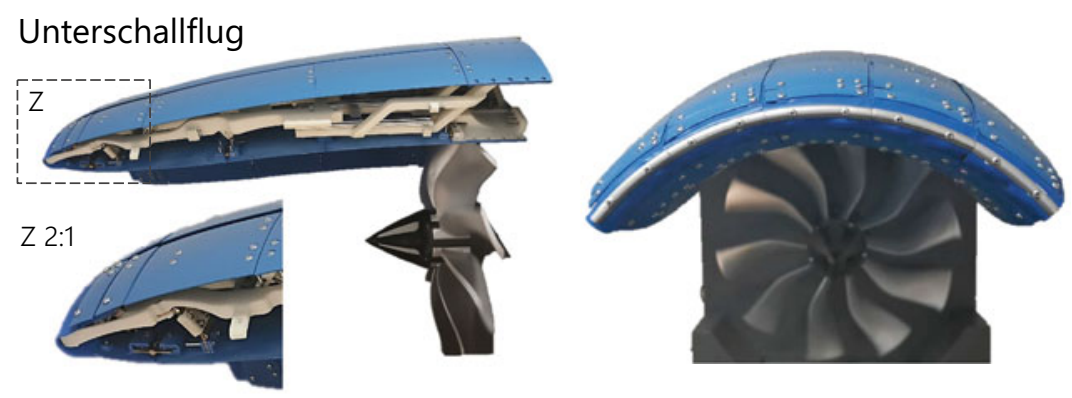

Überschallflug
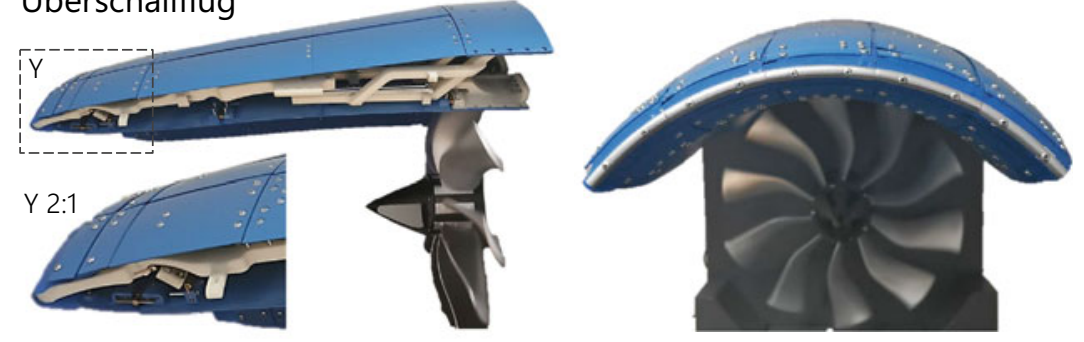

Abbildung 4.62 Zweiter Funktionsdemonstrator

Der Bau des Prototyps verdeutlicht die erhöhte Komplexität des ausgewählten Konzepts, die zum Einstellen aerodynamisch effizienter Geometrien erforderlich ist. Die Aktorik muss für die Konturvariation neben den Strömungskräften auch die nicht zu vernachlässigende Trägheit und Reibung des Systems überwinden. Diese Faktoren werden durch den Verkleinerungsmaßstab und die verhältnismäßig großen Bauteiltoleranzen jedoch verstärkt. Die finalen Spalte und Stufen auf der Einlasskontur sind von geringem Ausmaß.

\subsubsection{Vogelschlaguntersuchungen}

Durch die Erstellung des zweiten Funktionsdemonstrators wurde der Reifegrad TRL 3 des Konzepts nachgewiesen, jedoch auch seine hohe Komplexität verdeutlicht. Um deren Einfluss auf die Sicherheit im Vogelschlagfall zu bestimmen, werden nachfolgend vorläufige Ergebnisse einer Vogelschlaganalyse des erarbeiteten Konzepts vorgestellt, vgl. Selleng [362]. Diese wurde mit Hilfe des Simulationsprogramms LS-Dyna durchgeführt. 
Für die Untersuchung von Vogelschlägen hat sich das hydrodynamische Modell nach Wilbeck [363] durchgesetzt [309, S. 50-52]. Dieses Modell basiert auf der Annahme, dass sich der Vogel beim Aufprall auf einen festen Körper wie ein Fluid verhält [309, S. 50-52]. Somit fließt er beim Auftritt auf eine Komponente an dieser entlang, anstatt sie, wie ein Projektil, zu durchbrechen. Dieses Verhalten wird durch die Dichtedifferenz zwischen der metallischen Auftrittskomponente und dem Vogel mit deutlich geringerer Dichte begründet [309, S. 51]. Ein ähnliches Beispiel stellen Insekten auf Autofrontscheiben dar.

Im Rahmen numerischer Untersuchungen findet häufig das SPH-Modell (Smoothed Particle Hydrodynamics, geglättete Teilchen-Hydrodynamik) Anwendung [309, S. 127]. Dieses vereint gute Eigenschaften für Robustheit und Genauigkeit [309, S. 131]. Deshalb wurde es auch in der vorliegenden Untersuchung verwendet, vgl. Selleng [362]. Erforderliche Randbedingungen, wie die Masse, Größe und Geschwindigkeit des Vogels können der Fachliteratur [364, S. 2083], [309, S. 143-144] entnommen werden. Üblicherweise werden hierbei Vögel mit ca. $2 \mathrm{~kg}$ Masse und einer Relativgeschwindigkeit von etwa $130 \mathrm{~m} / \mathrm{s}$ modelliert, vgl. Anhang A.1.1. Die grundlegende Validierung des modellierten Vogels kann mittels der erzeugten Spannungsverteilung beim Aufschlag auf eine Platte erfolgen [309, S. 210].

Der obere Teil von Abbildung 4.63 stellt die Verformung des Referenzeinlasses aus Aluminium mit $3 \mathrm{~mm}$ Wandstärke dar, vgl. Anhang A.6.1. Wie zu erkennen ist, hält die Einlassstruktur den wirkenden Beanspruchungen ohne Rissbildung stand. Jedoch treten plastische Verformungen bis etwa $80 \mathrm{~mm}$ auf.

Die Simulation des Vogelschlags auf einen einzelnen $15^{\circ}$-Sektor des variablen Einlasskonzepts einschließlich des vollständigen umlaufenden Stellrings zeigt ein ähnliches Ausmaß maximaler Verformungen auf, vgl. Abbildung 4.63 mittig und unten. Es ist kein Versagen mehrerer Gelenkverbindungen und somit kein Verlust von Einlasskomponenten zu beobachten, wodurch der redundante Haltemechanismus für die Segmente nicht erforderlich wäre, vgl. Abschnitt 4.5.1. Weiterhin würden die benachbarten Sektoren den vom Vogelschlag betroffenen Sektor strukturell verstärken. Die benachbarten Sektoren wurden aufgrund des hohen Rechenaufwands vorerst nicht betrachtet.

Der Abgleich der präsentierten Ergebnisse mit der Fachliteratur [309], [363], [365], [366] zeigt eine gute Übereinstimmung. Somit geben die Ergebnisse erste Hinweise auf das strukturelle Verhalten des Einlasses im Vogelschlagfall. Für zuverlässige Aussagen diesbezüglich sind jedoch weitere Untersuchungen und reale Vogelschlagversuche erforderlich. 


\section{Starrer Referenzeinlass}
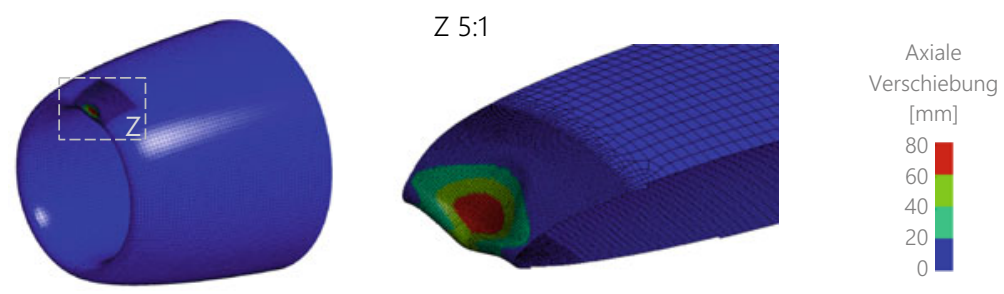

Einzelsektor des variablen Einlasses mit vollständigem Stellring
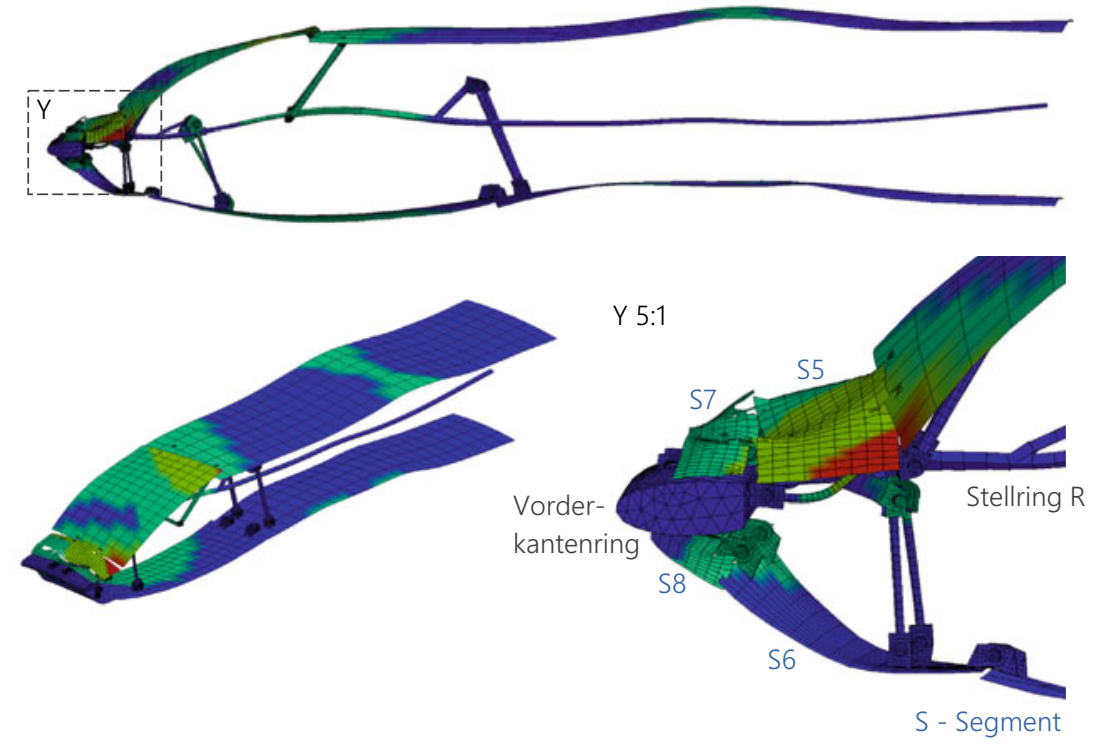

Abbildung 4.63 Vogelschlaguntersuchungen

Für metallische Drohnen wäre die hydrodynamische Annahme aufgrund der deutlich höheren Dichte unzulässig. Die Dichte von Drohnen ist im Bereich der Dichte der Komponenten, auf die sie aufschlägt und somit die Wirkung beim Aufschlag eher vergleichbar mit einem Projektil. Folglich existiert eine erhöhte Gefahr für strukturelles Versagen im Fall einer Kollision, auch unter Beachtung der häufig deutlich unter $2 \mathrm{~kg}$ liegenden Masse von Drohnen. 


\subsection{Abschätzung des Technologie-Potenzials}

Die im Rahmen dieser Konzeptstudie durchgeführten Untersuchungen erlauben eine Abschätzung des grundlegenden Potenzials variabler Pitot-Einlässe im Allgemeinen und des erarbeiteten Konzepts im Besonderen. Neben den in Abschnitt 2.4.1 vorgestellten Vorteilen variabler Pitot-Einlässe haben die folgenden Gesichtspunkte einen Einfluss auf das Potenzial dieser Technologie:

- Funktionalität,

- Innovativität,

- Sicherheit,

- Komplexität und

- Masse.

Variable Pitot-Einlässe können durch Variation der Geometrie in verschiedenen Flugphasen positive Effekte bewirken. So ermöglichen sie einerseits eine erhöhte Sicherheit und Erweiterung des Betriebsbereichs bei langsamen Fluggeschwindigkeiten. Andererseits unterstützen sie einen effizienteren Reiseflugbetrieb, sowohl im Unterschall als auch im Überschall. Das erarbeitete Konzept zielt auf eine Effizienzerhöhung im Reiseflugbetrieb ab. Diese wird durch eine innovative Variation der Einlasskontur zwischen Unterschall- und Überschallbetrieb erreicht. Dabei erfolgt eine Geometrievariation von Einlasslippe, Diffusor und Gondelvorkörper durch Verschieben eines geschlossenen Vorderkantenrings sowie starrer Komponenten der axial und über den Umfang segmentierten Einlasskontur.

Existierende Patente, die ebenfalls starre Kontursegmente verschieben, [30], [31], [158], [214] erlauben nur eine deutlich eingeschränktere Variation der Einlasskontur und sind darüber hinaus anfällig für sicherheitskritische Probleme, vgl. Abschnitt 2.4.2. Hierzu ist die Anfälligkeit der Vorderkante gegenüber Vogelschlag [31], [214] zu erwähnen, die im vorliegenden Konzept durch den geschlossenen Vorderkantenring minimiert wird. Dabei wird im Gegensatz zu einem anderen Konzept mit verschiebbarem Vorderkantenring [30] die Spaltbildung im Bereich der Vorderkante durch gleitende Gelenkverbindungen des Vorderkantenrings mit den Kontursegmenten des Einlasses verhindert, vgl. Abschnitt 4.5.1. Die Entstehung von Umfangsspalten zwischen den Kontursegmenten bei der Querschnittsvariation wird durch Überlappung der Segmente unter Nutzung linearer Segmentverlängerungen erreicht, vgl. Abschnitt 4.2.4. Für diese konzeptuellen Neuerungen bezüglich variabler Pitot-Einlässe erfolgte zudem als Bestandteil der vorliegenden Konzeptstudie eine Patentanmeldung. Das zugehörige Patentierungsverfahren ist zum gegenwärtigen Zeitpunkt noch nicht abgeschlossen. 
Das erarbeitete Konzept kann die Sicherheit des Flugbetriebs bei allen identifizierten sicherheitskritischen Ereignissen gewährleisten. So werden Strömungsablösungen im Startfall und beim Steigflug durch die Unterschallgeometrie des Einlasses umgangen, vgl. Abschnitt 4.4.3. Die Sicherheit bei Vogelschlag ist durch die Dimensionierung der Konzeptkomponenten gegeben, vgl. Abschnitt 4.5.5 und Anhang A.6. Zusätzlich kann der Verlust von Kontursegmenten durch einen redundanten Haltemechanismus verhindert werden, vgl. Abschnitt 4.5.1. Eine mögliche Vereisung und deren negative Konsequenzen werden durch ein elektrothermisches Eisschutzsystem vermieden. Die Gefahr einer Feuerentstehung innerhalb der Einlasszone ist durch die ausschließliche Verwendung elektrischer Teilsysteme für Aktorik und Eisschutz minimal, vgl. Abschnitt 4.4.2. Die einzig möglichen brennbaren Fluide innerhalb der Einlasszone wären somit die Schmiermittel der Stelltriebe, die durch Gehäuse vor möglichen Zündquellen geschützt sind, vgl. Abschnitt 4.4.1.

Die Integration einer zusätzlichen Funktion, in diesem Fall der Konturvariation, resultiert stets in einer Erhöhung der Komponentenanzahl und der Komplexität eines Systems. Durch die erhöhte Anzahl an Teilkomponenten sind im weiteren Verlauf der Entwicklung verhältnismäßig hohe Zuverlässigkeitsanforderungen an die einzelnen Komponenten zu stellen. Beim Einhalten dieser Anforderungen kann eine Ausfallwahrscheinlichkeit variabler Einlässe auf dem Niveau starrer Einlässe erreicht werden. Zusätzlich ist ein erhöhter Inspektions- und Wartungsaufwand für das variable Einlasssystem und insbesondere den verwendeten Variationsmechanismus erforderlich. Darüber hinaus ist davon auszugehen, dass nach Vogelschlagereignissen die meisten Komponenten des Einlasses ausgetauscht werden müssen.

Die Masse der dimensionierten Komponenten des Konzepts beträgt ungefähr $172 \mathrm{~kg}$, vgl. Tabelle 4.20. Hierzu zu ergänzen sind noch die Massen

- des Aktorkontrollsystems, des Feststellmechanismus und zugehöriger Leitungen,

- des Eisschutzsystems mit ca. 20 kg, vgl. Abschnitt 2.3.2,

- der Akustikauskleidungen, äquivalent zu herkömmlichen Einlässen,

- eines möglichen redundanten Segmenthaltemechanismus sowie

- zusätzlicher Bauteile, wie Schrauben, Muttern und Abdichtungen.

Herkömmliche Pitot-Einlässe verwenden zumeist ein schweres Heißlufteisschutzsystem. Zudem bringen die starre Außenkontur und deren Versteifungselemente ebenfalls eine gewisse Masse mit sich. Folglich ist davon auszugehen, dass die zusätzliche Masse durch Verwendung variabler Einlässe die geforderten $500 \mathrm{~kg}$ 
deutlich unterschreiten wird. Dadurch könnte sich der erzielte Reichweitengewinn bei Verwendung variabler Einlässe in Überschallflugzeugen bis Mach 1,6 anstelle der identifizierten $20 \%$ auf Werte im Bereich von $30 \%$ belaufen, vgl. Abschnitt 4.4.3. Dieser sehr gute Wert kann jedoch durch Spalte und Stufen entlang der Kontur beeinträchtigt werden und ist mittels weiterer aerodynamischer Untersuchungen abzusichern, vgl. Abschnitt 4.5.1.

Das große identifizierte Anwendungspotenzial variabler Pitot-Einlässe für den Überschallflug bei Verwendung des erarbeiteten Konzepts kann zudem in Folgeentwicklungen weiter gesteigert werden. Eine Möglichkeit hierfür besteht in der Erweiterung des Flugzeugbetriebsbereichs, indem das Konzept eine zusätzliche, noch ablöseresistentere Geometrie für langsame Fluggeschwindigkeiten einstellt. Darüber hinaus könnte anstelle des Einlasses der gesamte Gondelvorkörper, einschließlich des maximalen Gondeldurchmessers variiert werden. Dadurch könnte der Luftwiderstand der gesamten Triebwerksgondel nochmals signifikant reduziert und auch der Reichweitengewinn gesteigert werden, vgl. Abschnitt 4.4.3. Diesbezüglich wurden während der Durchführung der Konzeptstudie einige Lösungsmöglichkeiten in einer weiteren laufenden Patentanmeldung (DE 102019125 038.2) skizziert.

Open Access Dieses Kapitel wird unter der Creative Commons Namensnennung 4.0 International Lizenz (http://creativecommons.org/licenses/by/4.0/deed.de) veröffentlicht, welche die Nutzung, Vervielfältigung, Bearbeitung, Verbreitung und Wiedergabe in jeglichem Medium und Format erlaubt, sofern Sie den/die ursprünglichen Autor(en) und die Quelle ordnungsgemäß nennen, einen Link zur Creative Commons Lizenz beifügen und angeben, ob Änderungen vorgenommen wurden.

Die in diesem Kapitel enthaltenen Bilder und sonstiges Drittmaterial unterliegen ebenfalls der genannten Creative Commons Lizenz, sofern sich aus der Abbildungslegende nichts anderes ergibt. Sofern das betreffende Material nicht unter der genannten Creative Commons Lizenz steht und die betreffende Handlung nicht nach gesetzlichen Vorschriften erlaubt ist, ist für die oben aufgeführten Weiterverwendungen des Materials die Einwilligung des jeweiligen Rechteinhabers einzuholen.

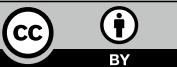

